



\section{PROSPECTIVE ASSESSMENT OF}

\section{ROBOTIC PELVIC FLOOR SURGERY}

Femke van Zanten 



\title{
PROSPECTIVE ASSESSMENT OF ROBOTIC PELVIC FLOOR SURGERY
}

\section{PROEFSCHRIFT}

\author{
ter verkrijging van \\ de graad van doctor aan de Universiteit Twente, \\ op gezag van de rector magnificus, \\ prof. dr. T.T.M. Palstra, \\ volgens besluit van het College voor Promoties \\ in het openbaar te verdedigen \\ op woensdag 8 januari 2020 om 14.45 uur
}

door

Femke van Zanten

geboren op 22 augustus 1988

te Zaanstad, Nederland 


\section{Dit proefschrift is goedgekeurd door:}

de promotor: Dhr. Prof. Dr. I.A.M.J. Broeders

de co-promotor: Dhr. Dr. S.E. Schraffordt Koops

Cover design: Rie Verhorevoort | rieverhorevoort.exto.nl

Print: Ridderprint | www.ridderprint.nl

Lay out: Marja van Zanten

ISBN: 978-90-365-4886-1

DOI: $10.3990 / 1.9789036548861$

The printing of this thesis was financially supported by Applied Medical, Meander Medical Center and University of Twente.

(C) 2019 Femke van Zanten, The Netherlands. All rights reserved. No parts of this thesis may be reproduced, stored in a retrieval system or transmitted in any form or by any means without permission of the author. Alle rechten voorbehouden. Niets uit deze uitgave mag worden vermenigvuldigd in enige vorm of op enige wijze zonder voorafgaande schriftelijke toestemming van de auteur. 


\section{Graduation committee}

Chair

Supervisor

Co-supervisor

\section{Committee members}

Prof. Dr. M.Y. Bongers

Prof. Dr. E.C.J. Consten

Prof. Dr. B.A. O’Reilly

MD FRCOG FRANZCOG FRCPI

Dr. H.W.R. Schreuder

Prof. Dr. Ir. S. Stramigioli

Prof. Dr. C.H. van der Vaart

Prof. Dr. Ir. R.M. Verdaasdonk
Prof. Dr. J.N. Kok, Twente University, Faculty of Electrical engineering, Mathematics and Computer Science, Enschede, The Netherlands.

Prof. Dr. I.A.M.J. Broeders, Twente University, Faculty of Electrical Engineering, Mathematics and Computer Science, Enschede, The Netherlands.

Dr. S.E. Schraffordt Koops, Meander Medical Center, Department of Gynaecology, Amersfoort, The Netherlands.

Department of Obstetrics and Gynaecology, GrowSchool for Oncology and Developmental Biology, Maastricht University, Maastricht, The Netherlands.

Department of Surgery, University Medical Center Groningen, University of Groningen, Groningen, The Netherlands.

Department of Obstetrics and Gynaecology, University College Dublin, Dublin, Ireland.

Department of Gynaecological Oncology, University Medical Center Utrecht, Utrecht, The Netherlands.

Department of Robotics and Mechatronics, University of Twente, Enschede, The Netherlands.

Department of Urogynaecology, University Medical Center Utrecht, Utrecht University, Utrecht, The Netherlands.

Department of Healthcare Technology Implementation, University of Twente, Enschede, The Netherlands. 


\section{Content}

Chapter $1 \quad$ General introduction and thesis outline

Part 1 Anatomic results, patient-reported outcomes and perioperative data

\begin{tabular}{ll}
\hline Chapter 2 & $\begin{array}{l}\text { Robot-assisted surgery for the management of apical } \\
\text { prolapse: a bi-centre prospective cohort study } \\
\end{array}$ \\
& British Journal of Obstetrics and Gynaecology \\
& Le19;126:1065-1073. \\
Chapter 3 & sacrocolpo(recto)pexy: a cumulative sum analysis \\
& American Journal of Obstetrics and Gynecology \\
& 2019;221:483.e1-11. \\
& Sexual function after robot-assisted prolapse surgery: \\
& a prospective study \\
& International Urogynecology Journal 2018;29:905-912.
\end{tabular}

Part 2 The sustainability of pelvic floor surgery

$\begin{array}{lll}\text { Chapter } 5 & \begin{array}{l}\text { Long-term anatomical and functional results of robot- } \\ \text { assisted pelvic floor surgery for the management of } \\ \text { multicompartment prolapse: a prospective study }\end{array} & 93 \\ & \begin{array}{l}\text { Diseases of the Colon \& Rectum 2019. } \\ \text { Robot-assisted sacrocolpopexy: not only for vaginal } \\ \text { Vhapter } 6\end{array} & 111 \\ & \text { vault suspension? An observational cohort study }\end{array}$

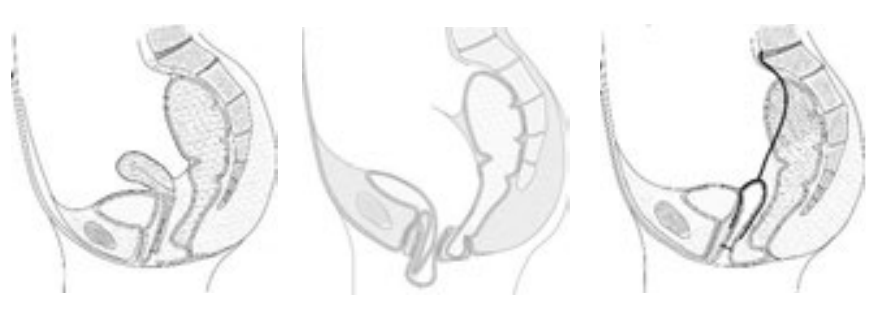


Part 3 Mesh-related complications

\begin{tabular}{lll}
\hline Chapter 7 & Mesh exposure after robot-assisted laparoscopic pelvic & 131 \\
& floor surgery: a prospective cohort study \\
& Journal of Minimally Invasive Gynecology \\
& 2019;26,636-642. \\
& Long-term mesh erosion rate following abdominal & 147 \\
Chapter 8 & robotic reconstructive pelvic floor surgery: a prospective \\
& study and overview of the literature \\
& International Urogynecology Journal 2019 \\
& Doi 10.1007/s00192-019-03990-1. \\
& Slechte sinaasappelnetjes, of is er meer onder de zon? \\
& English summary
\end{tabular}

Part 4 General discussion and summary

$\begin{array}{lll}\text { Chapter } 10 & \text { General discussion and future perspectives } & 185 \\ \text { Chapter } 11 & \text { Summary \& Samenvatting } & 199 \\ \text { Chapter } 12 & \text { Appendices } & 211 \\ & \text { A. Questionnaires } \\ & \text { B. Abbreviations } \\ \text { C. Acknowledgements - dankwoord } & \\ \text { D. Curriculum vitae } & \text { E. List of publications } \\ & \text { F. PhD Doctoral Education Program }\end{array}$

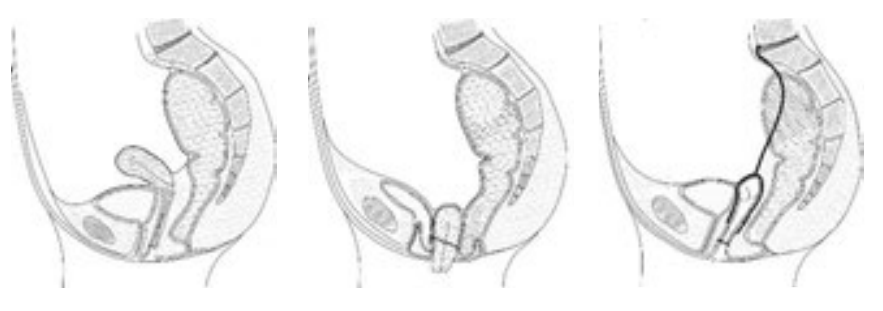





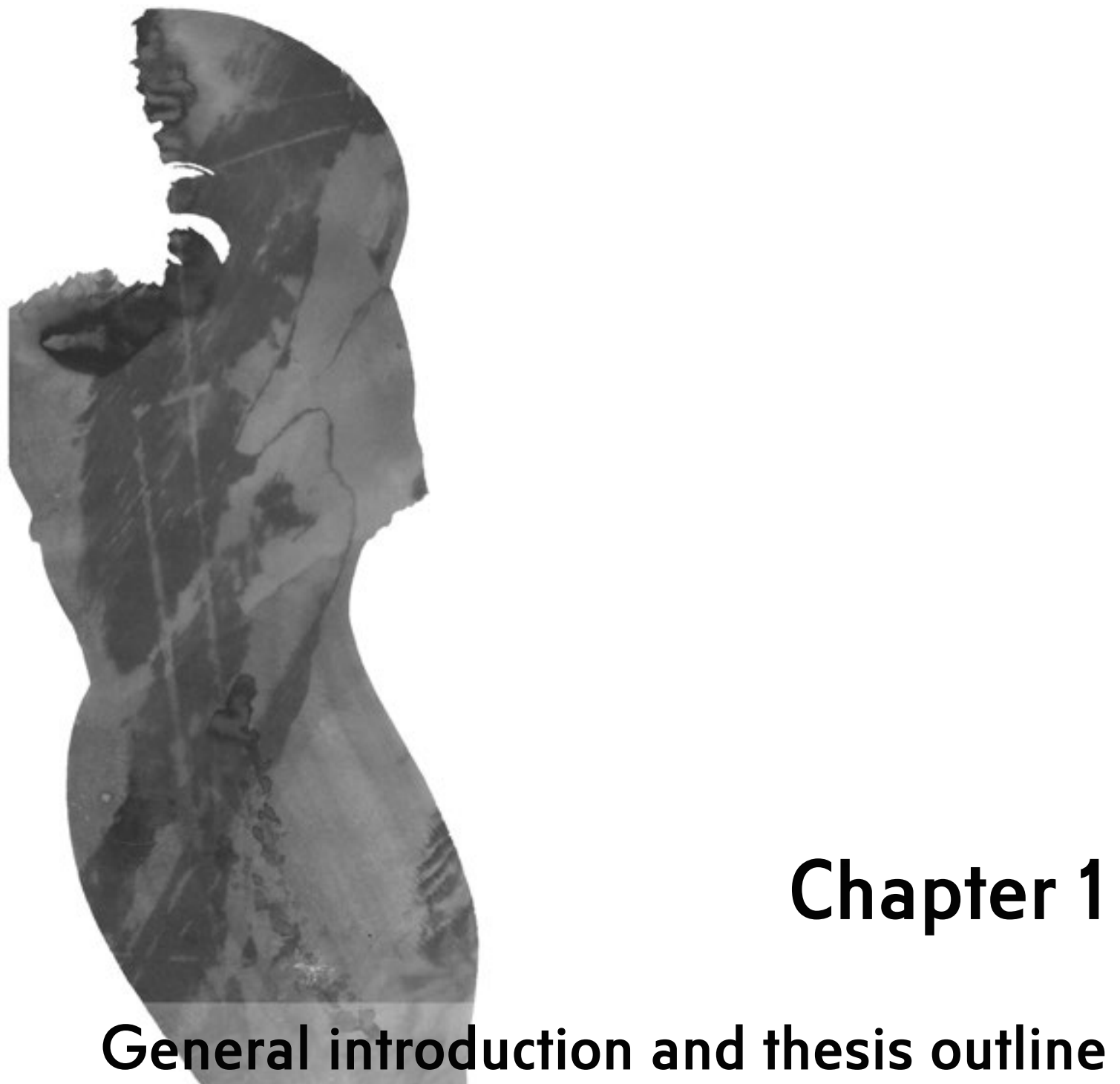




\section{Pelvic organ prolapse}

About 11-19\% of all women will undergo a surgical repair because of female pelvic floor prolapse (POP) and/or urine incontinence. ${ }^{1}$ In the Netherlands it is estimated that one out of five women will undergo a surgical repair for prolapse. ${ }^{2}$ POP is a condition where the pelvic organs fall down or 'slip' out of place. The word 'prolapse' is derived from the Latin word prolabi meaning 'to fall out'. It can include protruding of the bladder, uterus, vaginal cuff in post-hysterectomy patients, and the small or large bowel. This can lead to very severe symptoms and has a profoundly negative impact on the quality of life of those suffering from prolapse. ${ }^{3}$ The aetiology of POP is multifactorial and incompletely understood. Established risk factors are a history of vaginal delivery, advancing age, obesity and family history, but many other risk factors have been reported as well 14,5 (Figure 1).

Figure 1. Aetiological factors for pelvic floor dysfunction

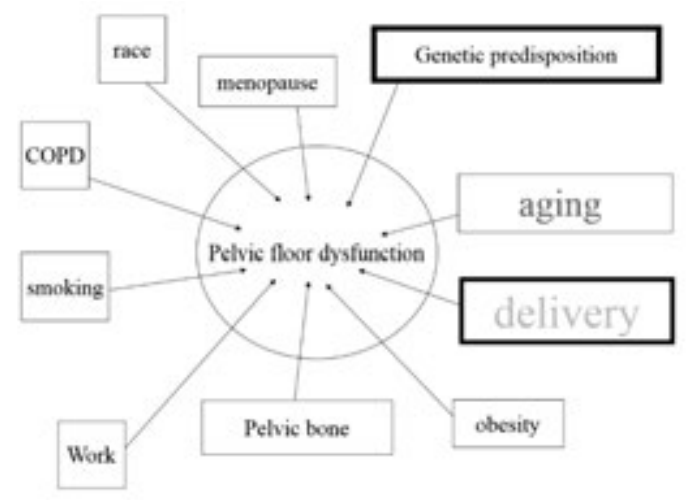

Economic costs of POP care are substantial and will rise significantly because of an aging population. It is estimated that during the time period of 2010 to 2050 POP will increase by $46 \% .{ }^{6}$ Costs-analysis in the care of POP is scarce, but direct costs of POP surgery were estimated to be $\$ 1,012$ million for 226.000 patient surgical procedures during 1997.7 Total annual surgical costs estimated in Europe varied from €81,030,907 to $€ 144,236,557$ in 2005 , based on the number of admissions (0.87-1.14 per 1000 women) for POP surgery. ${ }^{8}$ Careful evaluation of these procedures is therefore necessary to determine (cost) effectiveness. There are several surgical procedures and treatment options for women with POP. Recurrence rates, including vaginal vault prolapse, after a primary prolapse surgery are high and reoperation rates vary from $17-56 \%{ }^{9-11}$ Various 
procedures have been tried to correct vaginal vault prolapse, and the most successful to date has been sacrocolpopexy (SC). ${ }^{12,13}$ This procedure uses a mesh implant to suspend the prolapsed vaginal walls. It gives highly satisfactory results and results in fewer recurrences than vaginal approaches. SC is usually preformed in a minimally invasive fashion, which avoids the complications and longer return to daily activities that is related to open abdominal SC.12,14

The past decade, the safety of mesh surgery has been debated, because mesh-related complications were often seen after transvaginal surgical repair. The FDA issued a warning about mesh related complications in 2008 and $2011 .{ }^{15}$ Long-term evidence on the effectiveness and safety after mesh surgery are limited. ${ }^{16}$ The need for more longterm data on mesh-related outcomes is recommended and necessary. Simultaneously, there has been a shift in the technical aspect of the surgical approach. Laparoscopic surgery with robotic assistance has been performed increasingly. ${ }^{17}$ Therefore, this thesis was set up with the following major questions in mind. One, is sacrocolpo(recto)pexy performed with robotic assistance safe with good patient-reported outcomes on the mid and long-term, and two, what are the (long-term) mesh-related complications of this procedure?

\section{History}

Treatment of prolapse has been tried for centuries. The Egyptians described prolapse as 'falling of the womb' in the Kahun Papyrus. The first description of the usage of foreign body material for the repair of prolapse was the use of tantalum mesh in 1955, and in 1970 the first collagen mesh was described. ${ }^{18}$ The first description of the abdominal SC procedure was presented by the gynaecologist Lane in 1962.19 Several challenges in technique have been overcome in the past decades. Gynaecologists had to become more familiar with performing surgery in the rectovaginal space, instead of mainly in the vesicovaginal space. Throughout the years, the surgical approach shifted from open surgery to laparoscopic to robot-assisted surgery. After the FDA warnings, the use of minimally invasive sacrocolpopexy has increased over time. Use of mesh for vaginal suspensions has decreased. ${ }^{17}$ The robotic technique for surgical interventions was developed at the Stanford Research Institute, the United States (U.S.) Defense Department, and the National Aeronautics and Space Administration. The original cause was to treat wounded soldiers trough telesurgery. The operating surgeon could attempt surgery distant to the battlefield operating room. Practical performance was limited by a number of factors, such as band-with requirements. Robot-assisted laparoscopic surgery 
was further developed. It was cleared first by the FDA for urologic procedures in 2001 and in 2005 for gynaecologic procedures. Hysterectomy represents the second most common operative procedure in the U.S., with many still performed with an open abdominal incision. Robotic surgery will likely impact the gynaecological surgical care of women in the coming years. ${ }^{20}$

\section{Treatment of apical prolapse and surgical technique}

The first choice for vaginal apical prolapse treatment should be conservative. Up to $70 \%$ of women are satisfied with pessary treatment after one year. ${ }^{21}$ Specialised physiotherapy can aid in POP symptom relieve and improve quality of life in POPQ stage 1-2 prolapse. ${ }^{21}$ Regarding surgical treatment options, there are several possibilities, but the preferred technique is SC. Literature states success rates of $93-99 \% .^{12}$ Compared to vaginal apical repair procedures, ASC shows less recurrences and less dyspareunia, but this was balanced against a longer time to return to activities of daily life. ${ }^{12,22}$ The laparoscopic approach (LSC, laparoscopic sacrocolpopexy) has the advantages associated with laparoscopic surgery, such as reduced morbidity, shorter hospital stay and recovery, while maintaining similar clinical outcomes. ${ }^{23} \mathrm{~A}$ randomized trial, comparing 53 LSC with 55 total vaginal mesh repairs for vaginal vault prolapse, showed a clear benefit in favour of LSC with a higher satisfaction rate and objective success rate. The perioperative morbidity and reoperation rate in a 2 year follow-up was lower for LSC. ${ }^{24}$ In the Netherlands, treatment of vaginal vault prolapse varies among gynaecologists. ${ }^{25}$ Overall, sacrospinous fixation was used most, followed by LSC and RASC. ${ }^{25}$

\section{Assessing the outcome of prolapse surgery}

Determining success of prolapse surgery

Many definitions to describe success after POP repair have been used. The hymen appears to be an important cut-off point in the occurrence of symptoms. Women with prolapse beyond the hymen are more likely to report POP symptoms than women with prolapse at or above the hymenal remnants. ${ }^{26}$ In this thesis, prolapse more than 1 centimetre proximal to the hymen was therefore considered as successful. 
Simplified pelvic organ prolapse quantification

In this thesis, the simplified, or short version of the pelvic organ prolapse quantification system (sPOPQ) was used. ${ }^{27}$ The standard pelvic organ prolapse quantification (POPQ) exam was introduced in 1996 and recognized internationally by the International Continence Society, the American Urogynecologic Society, and the Society of Gynecologic Surgeons. They proposed a POP classification system that could be used internationally (Figure 2). ${ }^{28}$ This system describes nine vaginal landmarks to provide a clear description of vaginal prolapse in all compartments. It uses a ruler to measure the degree of prolapse in centimetres so that even small changes can be documented. The exam is specific and objective, with high intra- and inter-examiner agreement. ${ }^{27}$ In (clinical) practice, implementation has more challenges. Physicians used the POPQ only in 40\% clinically and $60 \%$ in research, and ordinal staging was often estimated. ${ }^{27}$ Simplifying the quantification system was therefore proposed to improve use in general practice. sPOPQ only examines four vaginal landmarks, while maintaining good inter-system association with the standard POPQ and good inter-examiner agreement. The four landmarks overlap with the standard POPQ, this is highlighted in Figure 2.

\section{Patients reported outcomes}

To assess improvement in patient-reported outcomes, validated questionnaires were used. Objective evaluations do not always correspond with the complaints which patients report. To evaluate success of the surgical intervention, the most important outcomes are the subjective results reported by patients. For this thesis, the urogenital distress inventory (UDI-6) was used to measure urinary symptoms, The Pelvic Floor Impact Questionnaire (PFIQ-7) for quality of life, and The Pelvic Organ Prolapse/Urinary Incontinence Sexual Questionnaire (PISQ-12) for sexual function.3,29-31 Questions regarding symptoms of obstructive defecation, faecal incontinence or vaginal bulge were asked separately in the questionnaire and during consultation.

\section{Robotic surgery}

In robotic surgery, the surgeon is seated in proximity to the patient, viewing the surgical site in a three-dimensional vision system and controlling laparoscopic instruments through handles that mimic the wrist motion and foot pedals while seated at a control panel (Figure 3). Robotic surgery has several advantages. Specifically for RASC, a technique that includes frequent intracorporeal suturing in the pelvis, it is an advantage 
to have a wide range of wrist motion. This freedom of motion is possible with robotic surgery and improves intracorporeal suturing and effects safety. ${ }^{32}$ Additionally, the 3D vision and complete camera control improve the vision of the surgeon. Tremors can be filtered out. Many laparoscopic surgeons report musculoskeletal disorders. ${ }^{33}$ Robotic

Figure 2. Pelvic organ prolapse quantification: standard versus simplified quantification

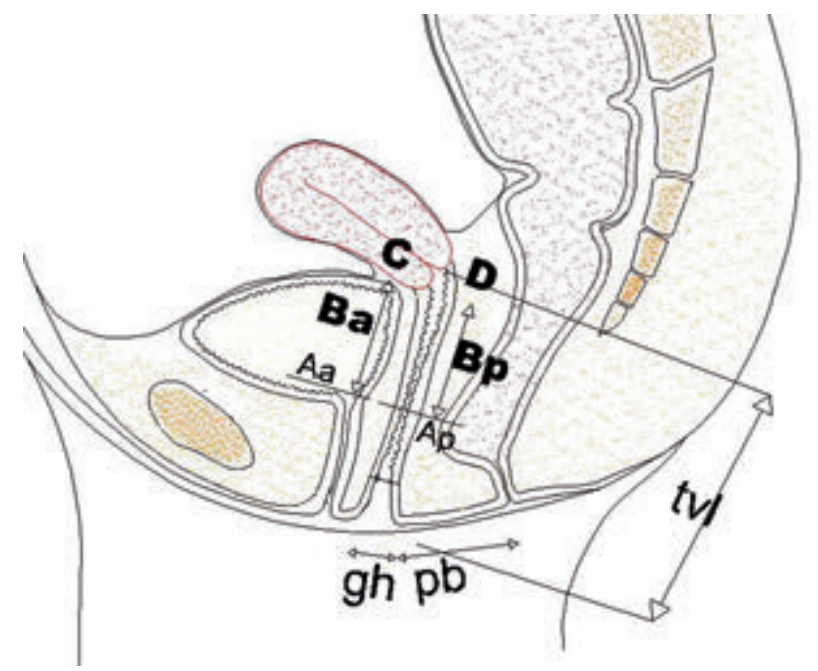

The nine landmarks of the standard pelvic organ prolapse quantification (POPQ) are listed in the figure. The four points of the simplified POPQ are highlighted in bolt letters: anterior vaginal segment (Ba), posterior vaginal segment (Bp), the cervix or vaginal cuff in hysterectomised patients $(\mathrm{C})$, posterior fornix (D) in non-hysterectomised patients. In patients with a hysterectomy in their history point D no longer exists. The SPOPQ has four stages: prolapse $>1 \mathrm{~cm}$ above of the hymenal remnants (stage 1 ); prolapse $1 \mathrm{~cm}$ above or $1 \mathrm{~cm}$ below the hymenal remnants (stage 2); prolapse $>1 \mathrm{~cm}$ past the hymenal remnants but does not represent complete vaginal vault or uterus eversion (stage 3); complete vaginal vault or uterus eversion (stage 4). Stage 0 does not exist in the simplified POPQ system.

surgery minimizes surgeon fatigue. As LSC can last up to three to four hours, the ergonomic position of the robotic console is beneficial for the physical stress of surgeons. ${ }^{34}$ The robot can offer optimized ergonomics, as the surgeon is seated on a chair, while arms are supported by armrests. Height of the chair and the angel of the view panel can be adjusted. The use of robotics has increased rapidly and intelligent surgery is more common than ever. Human performance is mixed with robotic supportive technique to improve surgical precision and convenience. Finally, robots can be used for simulation and training. One of the disadvantages of robotic surgery is the high costs, related to purchasing the robot and maintenance costs. ${ }^{35}$ Future 
developments in robotic surgery can possibly minimalize costs in the future. Improvement of ergonomics could possibly prevent sick leave in surgeons due to reduced musculoskeletal disorders. Other challenges regarding robotic surgery are the lack of haptic feedback and the correct assessment of mesh tension in the steep Trendelenburg position. This steep Trendelenburg position also requires a different anaesthetic plan. Ventilation of the patient in this position can be difficult.

\section{Mesh surgery}

The reason to use implants in pelvic floor surgery is multifactorial. It replaces insufficient tissue, and it provides additional support by multiplying the surface of support. Finally, it introduces new tissue support. We provide an overview of articles on RASC describing mesh exposure after a minimum follow-up duration of 12 months (Table 1). ${ }^{39-70}$ Subjective and objective outcomes of these studies are listed as well. Most studies solely describe success rates of the apical compartment. Whereas the most common type of recurrence of POP is situated in the anterior-compartment. ${ }^{36}$ Studies were highly heterogeneous. In cases where the uterus was present, different methods were used, varying from total hysterectomy, subtotal hysterectomy or hysteropexy (Table 1). There was a high range of loss to follow-up between studies. In total, 32 studies described 1966 patients. Objective success for the apical compartment varied from $92-100 \%$. The cumulative incidence of mesh complications was 63 (3.2\%, range 0$13.3 \%)$. Four studies examined postoperatively more than 100 patients. ${ }^{56,58,68,69}$ They reported a mesh exposure rate of $0 \%, 0 \%, 6.1 \%$ and $6.1 \%$. The study with the longest follow-up of 72 months showed $2.9 \%$ mesh reported complications. ${ }^{62}$ Most studies used a polypropylene mesh, which is the graft of first choice. ${ }^{13}$

As LSC has been performed most often, more long-term results are available for this type of surgery. Paqueé et al. (2019) described a prospective cohort of LSC. Patients with a minimum of 1.5 years of follow-up were included. 185 patients of 331 were seen for follow-up with a physical examination and interview and 95 additional patients were interviewed only. ${ }^{37}$ Reinterventions for prolapse occurred in $3.3 \%$ of patients and reoperations for graft-related complications in $7 \%$ of patients. Eighty-three percent of patients reported improvement based on the Patient Global Impression of Change score (PGI-I). In a large retrospective cohort of 660 patients who underwent LSC with median time from surgery of 4 years and 3 months, 5 patients $(0.7 \%)$ with vaginal mesh exposures and 4 with $(0.6 \%)$ suture erosions were identified.38 PGI-I scores were 

much or much better.

Figure 3. Surgical technique
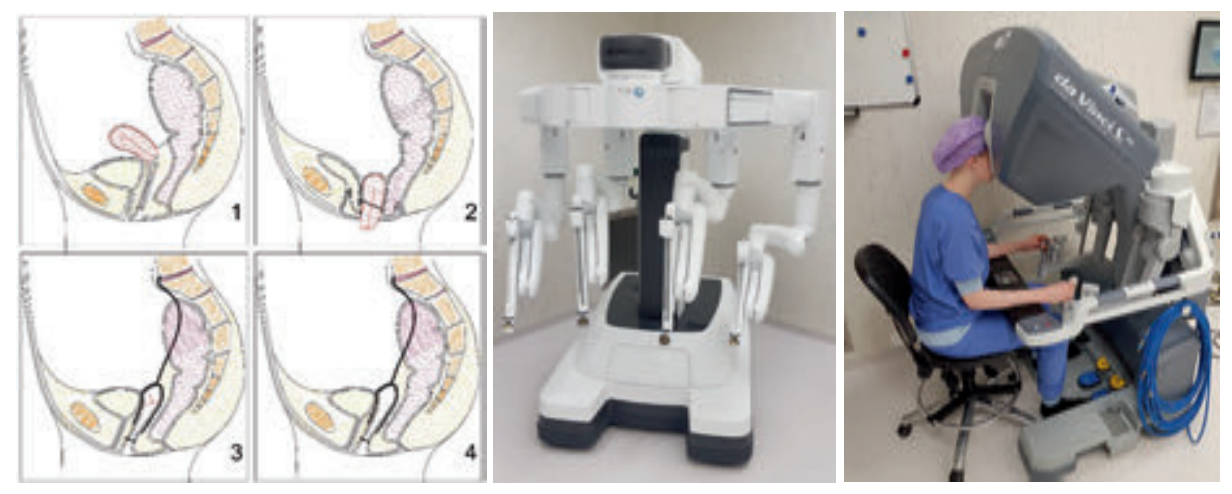

The da Vinci robot is an integrated computer-based system (right figure, da Vinci Si; middle figure, da Vinci Xi). It consists of 3 interactive robotic arms, 1 camera arm and 1 assistant port. Port placement is performed in a ' $W$ ' configuration. The camera port may be placed umbilical through a Veress needle (open technique on indication). After the camera port is placed, a pneumoperitoneum is created $(15 \mathrm{~mm} \mathrm{Hg})$ and the $0^{\circ}$ camera is introduced. Adequate port positioning is important because the robotic instruments must adequately reach the pelvis and promontory. Collision of the arms must be prevented. Before docking, the patient is placed in Trendelenburg position with the patient in Yellofin stirrups. The robot is then side docked parallel to the bed on the left side. The surgeon takes place in the robot console (remote-control unit). In robotic surgery the motions of the surgeon at the remote-control unit are replicated by the robotic arms placed within the patient. Its instruments provide the same flexibility as the human wrist.

Surgical procedure. The peritoneum is incised at the vaginal apex. An anterior plane is created between the bladder and the vagina and a posterior plane between the posterior vaginal wall and rectum. After retraction of the bowels, the sacral promontory is identified just below the bifurcation of the common iliac arteries. Awareness of the left and right common iliac vein, sacral vessels and right ureter is important when identifying and clearing the anterior longitudinal ligament of the promontory. The peritoneum can then be incised and opened from promontory to apex of the vagina. The mesh consists of two separate meshes that are configured to a ' $\mathrm{Y}$ ' shape intracorporeally. One mesh is placed on the anterior vaginal wall and the other longer mesh on the posterior vaginal wall after which the two meshes are attached together. The proximal end of the mesh is secured to the promontory with tackers or nonresorbable sutures. The final step is retroperitonealisation of the peritoneum over the mesh with a V-loc suture.

In case of a supracervical hysterectomy, this is the first step of the procedure. The cervix is identified and marked caudal of the uterosacral ligaments. Bipolar coagulation of the round and broad ligaments is performed on both sites. Lateral dissection is then performed until the marked part of the cervix after which the cervix is cut through with electrocautery. After this, a sacrocolpopexy is performed in a similar fashion as described earlier. The uterus will be removed through an endocatch bag to prevent spreading of a possible undiagnosed carcinoma. A small uterus can be removed in parts through the $12 \mathrm{~mm}$ assistant port without spill. If the uterus is larger, a morcellation device can be used to reduce the volume of the uterus. Recently bilateral salpingectomy is offered to patients to perform concomitantly for oncological reasons. Left figure: 1. No prolapse. 2. Stage 3 prolapse. 3. Robot-assisted (RA) supracervical hysterectomy with sacrocervicopexy. 4. RA sacrocolpopexy. The black line indicates the hymen. 


\section{Prospective cohort studies}

Several studies and guidelines underline the lack of long-term results after prolapse surgery, both vaginal as abdominal. Therefore, this study was set up as an observational prospective cohort study, to determine objective and reliable outcome measurement for care givers and patients. There is also need for randomized controlled trials (RCTs), comparing directly two (or more) different procedures with each other. In general, slower inclusion rates are seen with RCT's. ${ }^{71}$ We therefore chose to perform a long term prospective cohort study. 


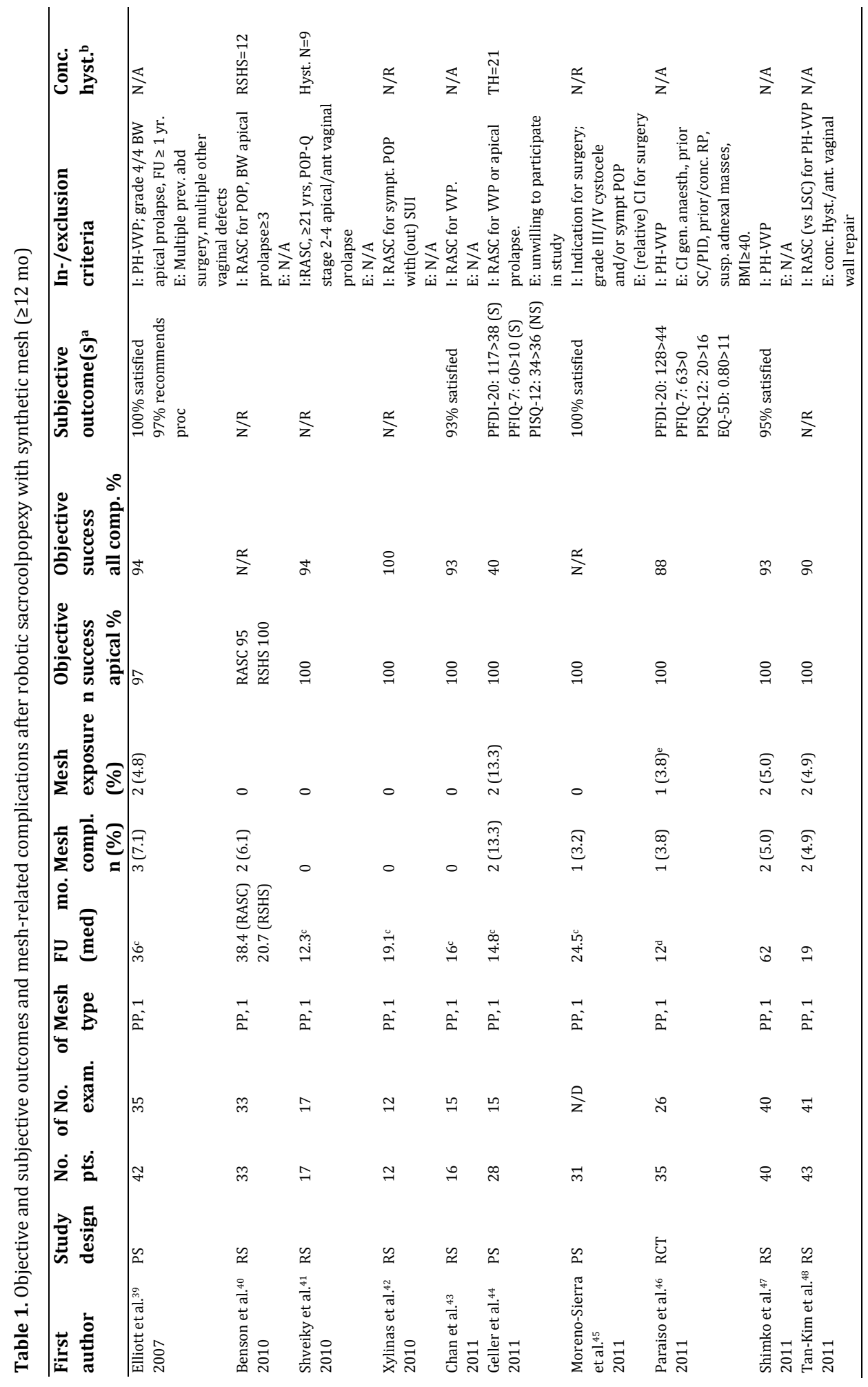




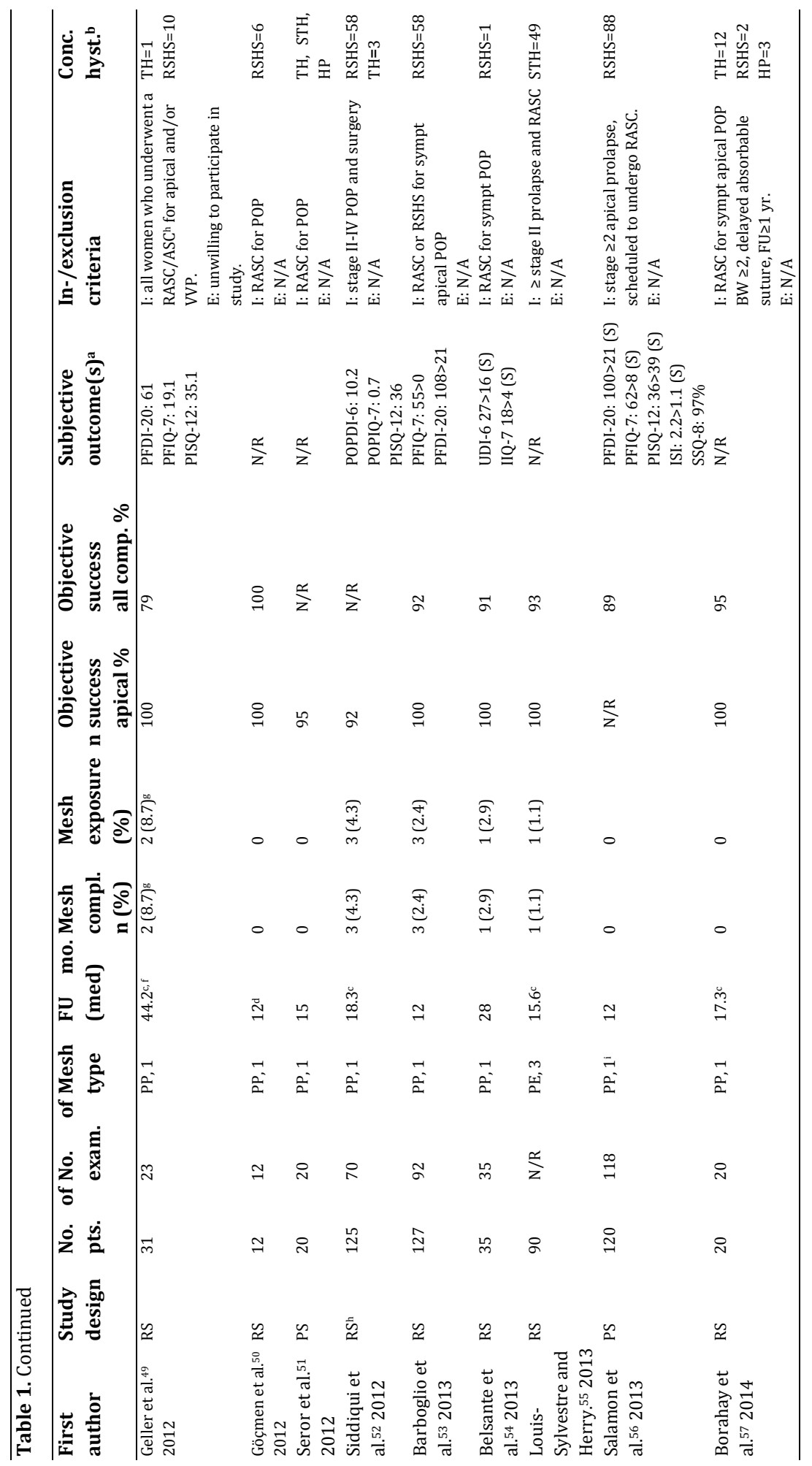




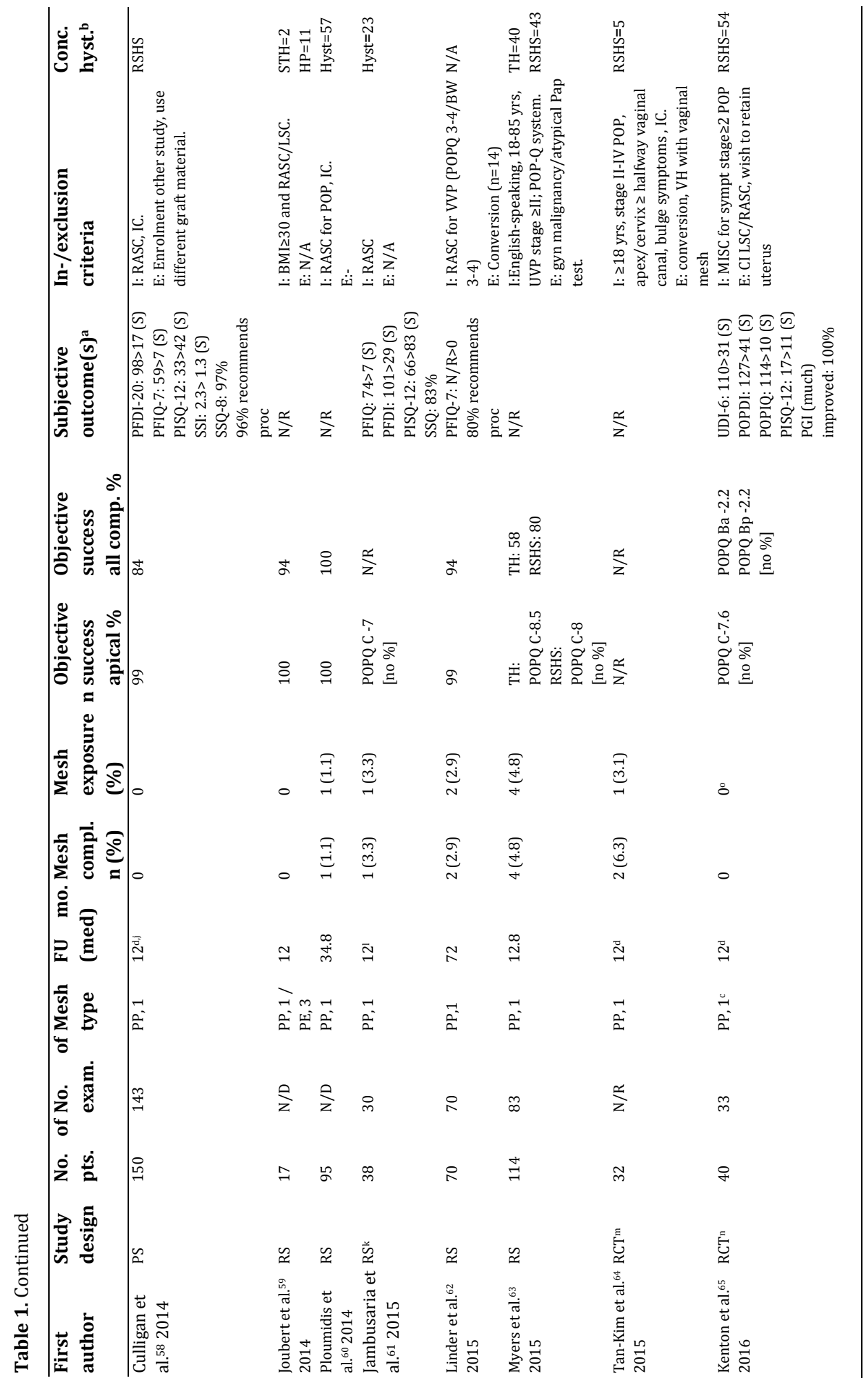




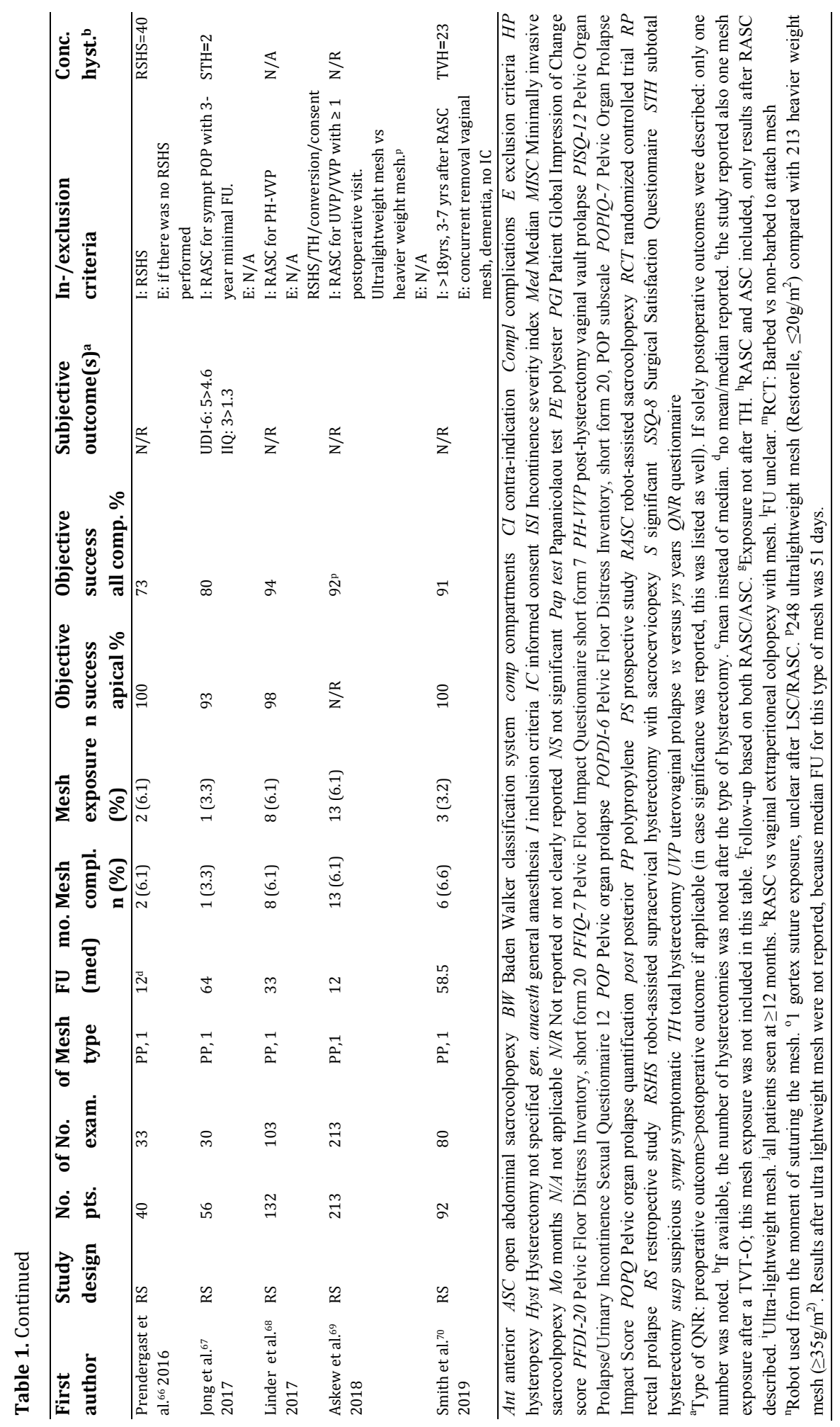




\section{Thesis outline}

Pelvic floor surgery is complex, and there are many ways to treat pelvic organ prolapse. This underlines the complexity of treating all deficiencies and different complaints of patients with POP. High recurrence rates after pelvic floor surgery indicate that there is a need to find alternative treatments for POP. Many different methods have been described making it hard to compare all treatment options with each other. Sacrocolpopexy has been increasingly used as it can be performed minimally invasive and has been associated with reduced recurrence rates compared to vaginal sacrospinous colpopexy. ${ }^{12}$ Responding to the need to develop new methods to treat POP, 'mesh kits' to treat prolapse via transvaginal routes have been developed. The earliest reports on these kits date from 2006. These types of surgery showed good anatomic results, but turned out to have serious mesh-related complications. Due to these complications, surgical repairs with mesh, not only transvaginal, but also trough the abdominal route as used in sacrocolpopexy, became a subject of debate. Although the abdominal route has been used for decades, this debate influenced the treatment of serious prolapse symptoms.

Against this backdrop, we developed a thesis to investigate the following subjects divided into three sections.

Part 1: What are realistic peri- and postoperative outcomes that can be expected when treating patients with robot-assisted laparoscopic sacrocolpopexy, with and without a supracervical hysterectomy?

Part 2: What are the long-term results of robot-assisted laparoscopic sacrocolpopexy for vaginal apical prolapse and sacrocolporectopexy for combined pelvic floor disorders?

Part 3: Is the use of mesh implants in sacrocolpopexy safe in the mid- and long-term?

To address these research questions, 7 studies were conducted. Chapter 2 describes a multicentre European study in which perioperative data for RASC with and without a subtotal hysterectomy was presented. One year postoperative anatomic results and patient-reported outcome measures were examined. With every new technique, surgeons undergo a learning curve. The learning curve for RASC and robot-assisted sacrocolporectopexy (RSCR) for multi-compartment prolapse was set out in chapter 3 . Sexual function after prolapse surgery is an under examined area. Therefore, we evaluated one year sexual function after RASC. This is outlined in chapter 4. In chapter 
5 and 6, the long-term objective outcomes and patients' reported symptoms after RASC and RSCR were described. Mid- and long-term complications specific to the use of mesh implants are investigated in chapter $\mathbf{7}$ and $\mathbf{8}$, and an overview of literature regarding mesh-related complications after minimally invasive SC is given. The results of these studies is presented in an editorial in chapter 9, to provide patients in The Netherlands and their caregivers with an overview of recent developments in the debate of mesh surgery. 


\section{References}

1. Smith FJ, Holman CDJ, Moorin RE, Tsokos N. Lifetime risk of undergoing surgery for pelvic organ prolapse. Obstet Gynecol. 2010;116:1096-1100.

2. de Boer TA, Slieker-ten Hove, Burger CW, Kluivers KB, Vierhout ME. The prevalence and factors associated with previous surgery for pelvic organ prolapse and/or urinary incontinence in a crosssectional study in The Netherlands. European J Obstet Gynecol Reprod Biol. 2011;158:343-349.

3. 't Hoen LA, Utomo E, Steensma AB, Blok BFM, Korfage IJ. The Pelvic Organ Prolapse/Urinary Incontinence Sexual Questionnaire (PISQ-12): validation of the Dutch version. Int Urogynecol J. 2015;26:1293-1303.

4. Jelovsek JE, Maher C, Barber MD. Pelvic organ prolapse. Lancet. 2007; 369: 1027-38

5. Slieker-ten Hove MCPh, Pool-Goudzwaard AL, Eijkemans MJC, Steegers-Theunissen PM, Burger CW, Vierhout ME. Symptomatic pelvic organ prolapse and possible risk factors in a general population. Am J Obstet Gynecol. 2009;200:184.e1-184.e7.

6. $\mathrm{Wu}$ JM, Hundley AF, Fulton RG, Myers ER. Forecasting the prevalence of pelvic floor disorders in U.S. Women: 2010 to 2050. Obstet Gynecol. 2009;114:1278-1283.

7. Subak LL, Waetjen LE, van den Eeden S, Thom DH, Vittinghoff E, Brown JS. Cost of pelvic organ prolapse surgery in the United States. Obstet Gynecol. 2001;98:646-651

8. Subramanian D, Szwarcensztein K, Mauskopf JA, Slack MC. Rate, type, and cost of pelvic organ prolapse surgery in Germany, France, and England. Eur J Obstet Gynecol Reprod Biol. 2009;144:177-81.

9. Clark AL, Gregory T, Smith VJ, Edwards R. Epidemiologic evaluation of reoperation for surgically treated pelvic organ prolapse and urinary incontinence. Am J Obstet Gynecol. 2003;189:1261-7

10. Olsen AL, Smith VJ, Bergstrom JO, Colling JC, Clark AL. Epidemiology of surgically managed pelvic organ prolapse and urinary incontinence. Obstetrics \& Gynaecology. 1997, 89, 501-506.

11. Denman MA, Gregory WT, Boyles SH, Smith V, Edwards SR, Clark AL. Reoperation 10 years after surgically managed pelvic organ prolapse and urinary incontinence. Am J Obstet Gynecol. 2008;198:555.e1-555.e5.

12. Maher C, Feiner B, Baessler K, Glazener CMA. Surgical management of pelvic organ prolapse in women. Cochrane Database of Syst Rev 2013;(4):CD004014:1-138.

13. Costantini E, Brubaker L, Cervigni L, Matthews CA, O'Reilly B, Rizk D, et al. Sacrocolpopexy for pelvic organ prolapse: evidence-based review and recommendations. Eur J Obstet Gynecol Reprod Biol. 2016;205:60-65.

14. Linder BJ, Ochhine JA, Habermann EB, Glasgow AE, Bews KA, Gershman B. A national contemporary analysis of perioperative outcomes of open versus minimally invasive sacrocolpopexy. J of Urology. 2018;200:862-867.

15. FDA. Food and Drug Administration. FDA safety communication: Urogynecologic surgical mesh : update on the safety and effectiveness of transvaginal placement for pelvic organ prolapse. Rev lit arts am. Accessed January 15, 2017. Available at: http://www.fda.gov/downloads/medicaldevices/safety/alertsandnotices/ucm262760.pdf

16. National Institute for Health and Care Excellence (NICE) guideline. Urinary incontinence and pelvic organ prolapse in women: management. Published: 2 April 2019. 
17. Slopnick EA, Petrikovets A, Sheyn D, Kim SP, Nguyen CT, Hijaz AK. Surgical trends and patient factors associated with the treatment of apical pelvic organ prolapse from a national sample. Int Urogynecol J. 2018;30:603-609.

18. Lensen EJ, Withagen MI, Kluivers KB, Milani AL, Vierhout ME. Surgical treatment of pelvic organ prolapse: a historical review with emphasis on the anterior compartment. Int Urogynecol J 2013;24:1593-602.

19. Lane FE. Repair of posthysterectomy vaginal-vault prolapse. Obstet Gynecol. 1962;20:72-77.

20. Holloway RW, Patel SD, Ahmed S. Robotic surgery in gynecology. Scandinavian J of Surgery. 2009;98:96-109.

21. Nationale Richtlijn Prolaps. Nationale richtlijnen database Dec 2014 (tevens. op Heelkunde \& NVOG.nl)

22. Rogers RG, Nolen TL, Weidner AC, Richter HE, Jelovsek JE, Shephard JP, et al. (for the NICHD Pelvic Floor Disorders Network) Open sacrocolpopexy and vaginal apical repair: retrospective comparison of success and serious complications. Int Urogynecol J. 2018;29:1101-1110.

23. Freeman RM, Pantazis K, Thomson A, Frappell J, Bombieri L, Moran P, et al. A randomised controlled trial of abdominal versus laparoscopic sacrocolpopexy for the treatment of posthysterectomy vaginal vault prolapse: LAS study. Int Urogynecol J. 2013;24:377-384.

24. Maher CF, Feiner B, DeCuyper EM, Nichlos CJ, Hickey KV, O'Rourke P. Laparoscopic sacral colpopexy vs. total vaginal mesh for the management of vaginal vault prolapse: a randomized trial. Am J Obstet Gynecol. 2011;204:360e1-7.

25. Vermeulen CKM, Coolen A-L WM, Spaans WA, Roovers J-P WR, Bongers MY. Treatment of vaginal vault prolapse in The Netherlands: a clinical practice survey. Int Urogynecol J. 2019;30:581-587

26. Barber MD, Brubaker L, Nygaard I, Wheeler TL, Schaffer J, Chen Z, et al. Defining success after surgery for pelvic organ prolapse. Obstet Gynecol 2009;114;600-609.

27. Swift S, Morris S, McKinnie V, Freeman R, Petri E, Scotti RJ, et al. Validation of a simplified technique for using the POPQ pelvic organ prolapse classification system. Int Urogynecol J Pelvic Floor Dysfunct. 2006;17:615-620.

28. Bump RC, Mattiasson A, Bø K, Brubaker LP, DeLancey JOL, Klarskov P, et al. The standardization of terminology of female pelvic organ prolapse and pelvic floor dysfunction. Am J Obstet gynecol. 1996;175:10-7.

29. van de Vaart CH, de Leeuw JRJ, Roovers JWR, Heintz APM. Measuring health-related quality of life in women with urogenital dysfunction: The Urogenital Distress Inventory and Incontinence Impact Questionnaire revisited. Neurourol and Urodynam. 2003;22:97-104.

30. Utomo E, Korfage IJ, Wildhagen MF, Steensma AB, Bangma CH, Blok BFM. Validation of the urogenital distress inventory (UDI-6) and incontinence impact questionnaire (IIQ-7) in a Dutch population. Neurourol Urodyn. 2015; 34:24-31.

31. Utomo E, Blok BF, Steensma AB, Korfage IJ. Validation of the Pelvic Floor Distress Inventory (PFDI20) and Pelvic Floor Impact Questionnaire (PFIQ-7) in a Dutch population. Int Urogynecol J 2014;25:531-44.

32. Stefanidis D, Wang F, Korndorffer JR Jr, Dunne JB, Scott DJ. Robotic assistance improves intracorporeal suturing performance and safety in the operating room while decreasing operator workload. Surg Endosc. 2010;24:377-382. 
33. Alleblas CJC, de Man AM, van den Haak L, Vierhout ME, Jansen FW, Nieboer TE. Prevalence of musculoskeletal disorders among surgeons. Performing Minimally Invasive Surgery. Ann Surg 2017;266:905-920.

34. Hubert N, Gilles M, Desbrosses K, Meyer JP, Felblinger J, Hubert J. Ergonomic assessment of the surgeon's physical workload during standard and robotic assisted laparoscopic procedures. Int J Med Robotics Comput Assist Surg. 2013;9: 142-147.

35. Anger JT, Mueller ER, Tarnay C, Smith B, Stroupe K, Rosenman A, et al. Robotic compared with laparoscopic sacrocolpopexy: a randomized controlled trial. Obstet Gynecol. 2014;123:5-12.

36. Durnea CM, Pergialiotis V, Duffy JMN, Bergstrom L, Elfituri A, Doumouchtsis SK, CHORUS, an International Collaboration for harmonising outcomes, research and standards in urogynaecology and women's health. A systematic review of outcome and outcome-measure reporting in randomised trials evaluating surgical interventions for anterior-compartment vaginal prolapse: a call to action to develop a core outcome set. Int Urogynecol J. 2018;29:1727-1745.

37. Pacqueé S, Nawapun K, Claerhout F, Werbrouck E, Veldman J, D'Hoore A, et al. Long-term assessment of a prospective cohort of patients undergoing laparoscopic sacrocolpopexy. Obstet Gynecol 2019;134:323-32.

38. Baines G, Price N, Jefferis H, Cartwright R, Jackson SR. Mesh-related complications of laparoscopic sacrocolpopexy. Int Urogyn J. 2019;30:1475-1481.

39. Elliott DS, Siddiqui SA, Chow GK. Assessment of the durability of robot-assisted laparoscopic sacrocolpopexy for treatment of vaginal vault prolapse. J Robot Surg. 2007;1:163-168.

40. Benson AD, Kramer BA, Wayment RO, Schwartz BF. Supracervical robotic-assisted laparoscopic sacrocolpopexy for pelvic organ prolapse. JSLS. 2010;14:525-530.

41. Shveiky D, Iglesia CB, Sokol AI, Kudish BI, Gutman RE. Robotic sacrocolpopexy versus vaginal colpopexy with mesh: choosing the right surgery for anterior and apical prolapse. Female Pelvic Med Reconstr Surg. 2010;16:121-127.

42. Xylinas E, Ouzaid I, Durand X, Ploussard G, Salomon L, Gillion N, et al. Robot-assisted laparoscopic sacral colpopexy: initial experience in a high-volume laparoscopic reference center. J Endourol. 2010;24:1985-1989.

43. Chan SSC, Pang SMW, Cheung TH, Cheung RYK, Chung TKH. Laparoscopic sacrocolpopexy for the treatment of vaginal vault prolapse: with or without robotic assistance. Hong Kong Med J. 2011;17:54-60.

44. Geller EJ, Parnell BA, Dunivan GC. Pelvic floor function before and after robotic sacrocolpopexy: one-year outcomes. J Minim Invasive Gynecol. 2011;18:322-327.

45. Moreno Sierra J, Ortiz Oshiro E, Fernandez Pérez C, Galante Romo I, Corral Rosillo J, Prieto Nogal S, et al. Long-term outcomes after robotic sacrocolpopexy in pelvic organ prolapse: prospective analysis. Urol Int. 2011;86:414-418.

46. Paraiso MFR, Jelovsek JE, Frick A, Chen CCG, Barber MD. Laparoscopic compared with robotic sacrocolpopexy for vaginal prolapse: a randomized controlled trial. Obstet Gynecol. 2011;118:1005-1013.

47. Shimko MS, Umbreit EC, Chow GK, Elliott DS. Long-term outcomes of robotic-assisted laparoscopic sacrocolpopexy with a minimum of three years follow-up. J Robot Surg. 2011;5:175-180. 
48. Tan-Kim J, Menefee SA, Luber KM, Nager CW, Lukacz ES. Robotic-assisted and laparoscopic sacrocolpopexy: comparing operative times, costs and outcomes. Female Pelvic Med Reconstr Surg. 2011;17:44-49.

49. Geller EJ, Parnell BA, Dunivan GC. Robotic vs abdominal sacrocolpopexy: 44-month pelvic floor outcomes. Urology. 2012;79:532-536.

50. Göçmen A, Sanlıkan F, Uçar MG. Robotic-assisted sacrocolpopexy/sacrocervicopexy repair of pelvic organ prolapse: initial experience. Arch Gynecol Obstet. 2012;285:683-688.

51. Seror J, Yates DR, Seringe E, Vaessen C, Bitker M-O, Chartier-Kastler E, et al. Prospective comparison of short-term functional outcomes obtained after pure laparoscopic and robotassisted laparoscopic sacrocolpopexy. World J Urol. 2012;30:393-398.

52. Siddiqui NY, Geller EJ, Visco AG. Symptomatic and anatomic 1-year outcomes after robotic and abdominal sacrocolpopexy. Am J Obstet Gynecol. 2012;206:435.e1-5.

53. Barboglio PG, Toler AJW, Triaca V. Robotic sacrocolpopexy for the management of pelvic organ prolapse: a review of midterm surgical and quality of life outcomes. Female Pelvic Med Reconstr Surg. 2013;20:38-43.

54. Belsante M, Murray S, Dillon B, Zimmern P. Mid term outcome of robotic mesh sacrocolpopexy. Can J Urol. 2013;20:6656-6661.

55. Louis-Sylvestre C, Herry M. Robotic-assisted laparoscopic sacrocolpopexy for stage III pelvic organ prolapse. Int Urogynecol J. 2013;24:731-733.

56. Salamon CG, Lewis C, Priestley J, Gurshumov E, Culligan PJ. Prospective study of an ultralightweight polypropylene Y mesh for robotic sacrocolpopexy. Int Urogynecol J. 2013;24:13711375.

57. Borahay MA, Oge T, Walsh TM, Patel PR, Rodriguez AM, Kilic GS. Outcomes of robotic sacrocolpopexy using barbed delayed absorbable sutures. J Minim Invasive Gynecol. 2014;21:412416.

58. Culligan PJ, Gurshumov E, Lewis C, Priestley JL, Komar J, Shah N, et al. Subjective and objective results 1 year after robotic sacrocolpopexy using a lightweight Y-mesh. Int Urogynecol J. 2014;25:731-735.

59. Joubert M, Thubert T, Lefranc J-P, Vaessen C, Chartier-Kastler, Deffieux X, et al. Comparison of functional outcomes with purely laparoscopic sacrocolpopexy and robot-assisted sacrocolpopexy in obese women. Progrès en Urol J l'Association Fr d'urologie la Société Fr d'urologie. 2014;24:1106-1113.

60. Ploumidis A, Spinoit A-F, De Naeyer G, Schatteman P, Gan M, Ficarra V, et al. Robot-assisted sacrocolpopexy for pelvic organ prolapse: surgical technique and outcomes at a single high-volume institution. Eur Urol. 2014;65:138-145.

61. Jambusaria LH, Murphy M, Lucente VR. One-year functional and anatomic outcomes of robotic sacrocolpopexy versus vaginal extraperitoneal colpopexy with mesh. Female Pelvic Med Reconstr Surg. 2015;21:87-92.

62. Linder BJ, Chow GK, Elliott DS. Long-term quality of life outcomes and retreatment rates after robotic sacrocolpopexy. Int J Urol. 2015;22:1155-1158..

63. Myers EM, Siff L, Osmundsen B, Geller E, Matthews CA. Differences in recurrent prolapse at 1 year after total vs supracervical hysterectomy and robotic sacrocolpopexy. Int Urogynecol J Pelvic Floor Dysfunct. 2015;26:585-589. 
64. Tan-Kim J, Nager CW, Grimes CL, Luber KM, Lukacz ES, Brown HW, et al. A randomized trial of vaginal mesh attachment techniques for minimally invasive sacrocolpopexy. Int Urogynecol J. 2015;26:649-656.

65. Kenton K, Mueller ER, Tarney C, Bresee C, Anger JT. One-year outcomes after minimally invasive sacrocolpopexy. Female Pelvic Med Reconstr Surg. 2016;22:382-384.

66. Prendergast E, Silver H, Johnson LL, Simon M, Feinglass J, Kielb S, et al. Anatomic outcomes of robotic assisted supracervical hysterectomy and concurrent sacrocolpopexy at a tertiary care institution at initial adaptation of the procedure. Female Pelvic Med Reconstr Surg. 2016;22:29-32.

67. Jong K. Klein T, Zimmern PE. Long-term outcomes of robotic mesh sacrocolpopexy. J Robotic Surg. 2018;12:455-460.

68. Linder BJ, Anand M, Klingele CJ, Trabuco EC, Gebhart JB, Occhino JA. Outcomes of robotic sacrocolpopexy using only absorbable suture for mesh fixation. Female Pelvic Med Reconstr Surg. 2017;23: 13-16.

69. Askew AL, Visco AG, Weidner AC, Truong T, Siddiqui NY, Bradley MS. Does mesh weight affect time to failure after robotic-assisted laparoscopic sacrocolpopexy? Female Pelvic Med Reconstr Surg. 2018;00:00-00.

70. Smith BC, Crisp CC, Kleeman SD, Yook E, Pauls RN. Uterosacral ligament suspension versus robotic sacrocolpopexy for treatment of apical pelvic organ prolapse. Female Pelvic Med Reconstr Surg. 2019;25: 93-98.

71. Collins S, Tulikangas P. Randomized trials in robotic surgery: a practical impossibility? Int Urogynecol J. 2010;21:1045-7. 





\section{Chapter 2}

Robot-assisted surgery for the management of apical prolapse: a bi-centre prospective cohort study

Femke van Zanten, Steven E. Schraffordt Koops, Orfhlaith E. O'Sullivan, Egbert Lenters, Ivo A.M.J. Broeders, Barry A. O'Reilly 


\section{Abstract}

Objective: Robot-assisted surgery is a recognised treatment for pelvic-organ prolapse. Many of the surgical subgroup outcomes for apical prolapse are reported together, leading to a paucity of homogenous data.

Design: Prospective observational cohort study (NCT01598467, clinicaltrials.gov) assessing outcomes for homogeneous subgroups of robot-assisted apical prolapse surgery.

Setting: Two European tertiary referral hospitals.

Population: Consecutive patients undergoing robot-assisted sacrocolpopexy (RASC) and supracervical hysterectomy with sacrocervicopexy (RSHS).

Methods: Anatomical cure (simplified Pelvic Organ Prolapse Quantification, sPOPQ, stage 1), subjective cure (symptoms of bulge), and quality of life (Pelvic Floor Impact Questionnaire, PFIQ-7).

Main outcome measures: Primary outcome: anatomical and subjective cure. Secondary outcomes: surgical safety and intraoperative variables.

Results: A total of 305 patients were included (RASC n=188; RSHS n=117). Twelve months follow-up was available for 144 (RASC 76.6\%) and 109 (RSHS 93.2\%) women. Anatomical success of the apical compartment occurred for 91\% (RASC) and in 99\% (RSHS) of the women. In all compartments, the success percentages were 67 and 65\%, respectively. Most recurrences were in the anterior compartment [15.7\% RASC (symptomatic 12.1\%); 22.9\% RSHS (symptomatic 4.8\%)]. Symptoms of bulge improved from 97.4 to $17.4 \%(p<0.0005)$. PFIQ-7 scores improved from $76.7 \pm 62.3$ to $13.5 \pm 31.1$ $(p<0.0005)$. The duration of surgery increased significantly for RSHS [183.1 \pm 38.2 versus $145.3 \pm 29.8(p<0.0005)]$. Intraoperative complications and conversion rates were low (RASC, 5.3 and 4.3\%; RSHS, 0.0 and 0.0\%). Four severe postoperative complications occurred after RASC (2.1\%) and one occurred after RSHS (1.6\%).

Conclusions: This is the largest reported prospective cohort study on robot-assisted apical prolapse surgery. Both procedures are safe, with durable results. 


\section{Introduction}

Over the last 15 years, the number of robot-assisted procedures performed for the treatment of female pelvic organ prolapse (POP) has increased. This is in part a result of the perceived simplification of complex laparoscopic manoeuvres and improved surgeon ergonomics. ${ }^{1,2}$ Despite this increase, there is a paucity of scientific papers reporting on anatomical outcomes and surgical safety for large groups after robot-assisted sacrocolpopexy. Furthermore, there should be a concern regarding the heterogeneity of surgical subgroups and techniques used within the published literature. ${ }^{3}$ In particular, most studies examining robot-assisted surgery for apical prolapse tend to combine surgery to support the vault (sacrocolpopexy) with surgery to support the cervix in patients with prior subtotal hysterectomy (sacrocervicopexy) and surgery to support the uterus (sacrohysteropexy) together. This makes it impossible to define outcomes relevant to each surgical subgroup, which has implications for how women are counselled regarding the selection of surgical approach. A recent paper by Anglim et al. reported on the factors influencing patient and surgeon decision making regarding uterine preservation or hysterectomy in the management of apical prolapse. ${ }^{4} \mathrm{~A}$ factor was literature bias: the Cochrane review in 2016 stated that the level of published evidence was poor. ${ }^{5}$ Furthermore, randomised controlled studies comparing robotic and laparoscopic apical repair include a low number of patients and acknowledge a diversity of surgeon experience, which makes a true assessment of outcomes very challenging. 6,7 The difficulty in performing a randomised control trial (RCT) in robotic surgery was described in an editorial by Collins et al. They describe the negative effect of patient, surgeon, and healthcare system bias on RCT, and highlight the role of prospective cohort studies in robot-assisted surgery. ${ }^{8}$ The strength of this prospective European bi-centre cohort study was to address these issues and, in particular, to provide results from homogeneous groups of procedures performed by robotically experienced surgeons. The main outcome measurements were long-term prolapse outcomes, intraoperative variables, and safety. 


\section{Methods}

\section{Study design}

The Prospective Assessment of Robotic Sacrocolpopexy: a European Bi-centre Cohort (PARSEC; NCT01598467, clinicaltrials.gov) was set up to collect data from European hospitals performing robot-assisted apical repair for symptomatic POP. Patients were not involved in the development of this research. All consecutive patients undergoing robot-assisted sacrocolpopexy (RASC) or robot-assisted supracervical hysterectomy with sacrocervicopexy (RSHS) between 2008 and 2016 in Cork University Maternity Hospital (Ireland) and in Meander Medical Centre (the Netherlands) were included. Both hospitals provide tertiary-level care for patients with POP. Preoperative counselling on alternative treatments and the risks and benefits of the procedure were discussed. Patients were consented accordingly.

Vaginal prolapse was defined using the simplified Pelvic Organ Prolapse Quantification (sPOPQ). ${ }^{9}$ sPOPQ describes four vaginal landmarks (Ba, anterior vaginal wall; Bp, posterior vaginal wall; $\mathrm{C}$, vaginal cuff/cervix; and $\mathrm{D}$, fornix posterior). ${ }^{9,10} \mathrm{SPOPQ}$ stage 1 describes either no prolapse or a minimal prolapse ( $>1 \mathrm{~cm}$ above the hymnal remnants). In stage 2 , the given point descends $1 \mathrm{~cm}$ above to $1 \mathrm{~cm}$ below the hymnal remnants. Stage 3 describes a prolapse that descends more than $1 \mathrm{~cm}$ beyond the hymenal remnants, but does not represent stage 4 , which includes complete vaginal vault eversion or complete procidentia uteri. Stage 0 is not defined in the SPOPQ system. Inclusion criteria were: symptomatic vaginal or uterine prolapse with an SPOPQ stage of $\geq 2$. Exclusion criteria were: age $<18$ years, poor health status with inability to undergo general anaesthesia, three or more previous laparotomies, planned pregnancy, and patients with a known pelvic malignancy. In patients who still retained their uterus, preoperative work-up for endometrial cancer or sarcoma was performed. An ultrasound and preoperative cervical cytology were performed for all patients.

The primary outcome measurement was anatomical cure, described as any SPOPQ score of $<2$. Patients were followed-up routinely with vaginal examination at 6 weeks and 12 months postoperatively, or at the onset of new symptoms. Failure was defined as any 
postoperative SPOPQ score of $\geq 2$ or retreatment. Recurrences were divided into symptomatic and asymptomatic recurrences and scored per compartment. Recurrences were considered symptomatic when there were symptoms of bulge (sensation of and/or seeing vaginal bulge) and/or retreatment [any POP reoperation (planned) or insertion of a vaginal pessary]. Patients received a questionnaire preoperatively and at follow-up (at 1 and at 5 years). The questionnaires included questions regarding the sensation of prolapse, quality of life (QoL) based on the Pelvic Floor Impact Questionnaire (PFIQ-7), ${ }^{11}$ urinary and defecation symptoms, the presence of dyspareunia, and the use of medication. The PFIQ-7 combines three QoL scales: the Urinary Impact Questionnaire, the Colorectal-Anal Impact Questionnaire, and the Pelvic Organ Prolapse Impact Questionnaire. Higher scores indicate a higher impact of symptoms on daily life (range $0-300$ ). The questionnaire was discussed during the 1-year postoperative visit or was returned by post if clinical consultation was not possible. If no vaginal examination (sPOPQ) was available and no questionnaire was completed at the 12-month time point, patients were considered lost to follow-up.

The secondary outcomes measured were safety of the procedure and intraoperative variables. 'Total surgery time' was defined as the time from first incision until the final suture was tied. Postoperative pain scores were measured on the first morning after surgery using the visual analogue scale (VAS, range 0-10). Intraoperative complications were scored using the following definition: 'Any deviation from the ideal intraoperative course occurring between skin incision and skin closure, including both surgery or anaesthesia-related complications'.12 A deviation from the planned intervention to manage unexpected intraoperative findings was not regarded as a complication (e.g. in the case of severe intra-abdominal adhesions). In the case of a conversion, an open abdominal sacrocolpopexy was performed unless otherwise specified. Postoperative complications occurring within 6 weeks were defined as 'early complications' and scored following the Clavien-Dindo Classification. ${ }^{13}$ Complications occurring after 6 weeks postoperatively were defined as 'late'. To date, there are no agreed standardised core outcome sets available for urogynaecology, and therefore specific recogniseed outcome measures were used in this study.

The surgical technique used for the RASC group is similar to that described by Clifton et al. ${ }^{14}$ Surgeries were performed by five gynaecologists. All surgeries were performed with the assistance of the da Vinci robot (Intuitive Surgical, Inc., Sunnyvale, CA, USA), and suspension was performed with type-1 polypropylene mesh [Prolene (Ethicon Inc., 
Somerville, NJ, USA) or Restorelle (Coloplast, Humlebaek, Denmark)]. Either a preformed Y-shaped mesh was used or two separate meshes, configured into a Y shape intracorporeally, were used. The mesh was attached distally using non-absorbable sutures (Ethibond, Ethicon, Johnson and Johnson or Gore-Tex, Gore medical W.L. Gore and Assoc, Inc, Flagstaff, AZ, USA). Proximal anchoring of the mesh to the sacral promontory was performed with titanium tacks (Autosuture Protack $5 \mathrm{~mm}$; Covidien, Mansfield, MA, USA) or non-absorbable sutures (Gore-tex). The peritoneum was approximated to cover the mesh completely using a 23-cm V-Loc suture (Covidien). The RSHS group had a supracervical hysterectomy performed prior to attaching the mesh to the anterior and posterior aspect of the cervix using the mesh and sutures as described for the RASC group. Additional procedures were performed when clinically indicated. As a result of recent scientific data on the pathophysiology of ovarian malignancy and spill in the case of sarcoma, concomitant salpingectomy and 'in bag' morcellation was performed from the year 2015 onwards. ${ }^{15,16}$

This study was performed in accordance with the ethical regulations of the Clinical Research Ethics Committee (CREC, University College Cork, Ireland) and the National Central Committee on Research Involving Human Subjects (CCMO, the Netherlands). No funding was received to conduct this study. Statistical analysis was performed using SPSS 22.0 (IBM Corp., Armonk, NY, USA). Data were presented as mean \pm standard deviation (SD) and median and interquartile range (IQR) for normally and non-normally distributed continuous values, respectively. In the case of SPOPQ values with only four stages, data were presented as means \pm SDs. Numbers and percentages were used for nominal and categorical values. Independent samples t-test, Mann-Whitney U-test, and chi-square test or Fisher's exact test were used to compare data for mean, median, and nominal values, respectively.

\section{Results}

In total 305 patients were included. One hundred and eighty-eight patients underwent RASC and 117 patients underwent RSHS (Figure 1). One hysteropexy was performed instead of supracervical hysterectomy for severe adhesions (0.9\%). The baseline characteristics of patients are depicted in Table 1 . When no hysterectomy was performed previously, patients were significantly younger (59.9 versus 63.1 years; $p=0.009$ ), and on average had a more severe preoperative prolapse of the anterior compartment than patients undergoing RASC (mean sPOPQ point Ba, stage 2.9 versus 
$2.5 ; p<0.0005)$. The median follow-up time was 12.6 and 14.8 months for RASC and RSHS, respectively. Ninety-five percent of all patients were seen 6 weeks postoperatively and $83 \%$ were seen 12 months postoperatively. Loss to follow-up per subtype of surgery is listed in Figure 1.

Figure 1. Flow chart for the follow-up of included patients

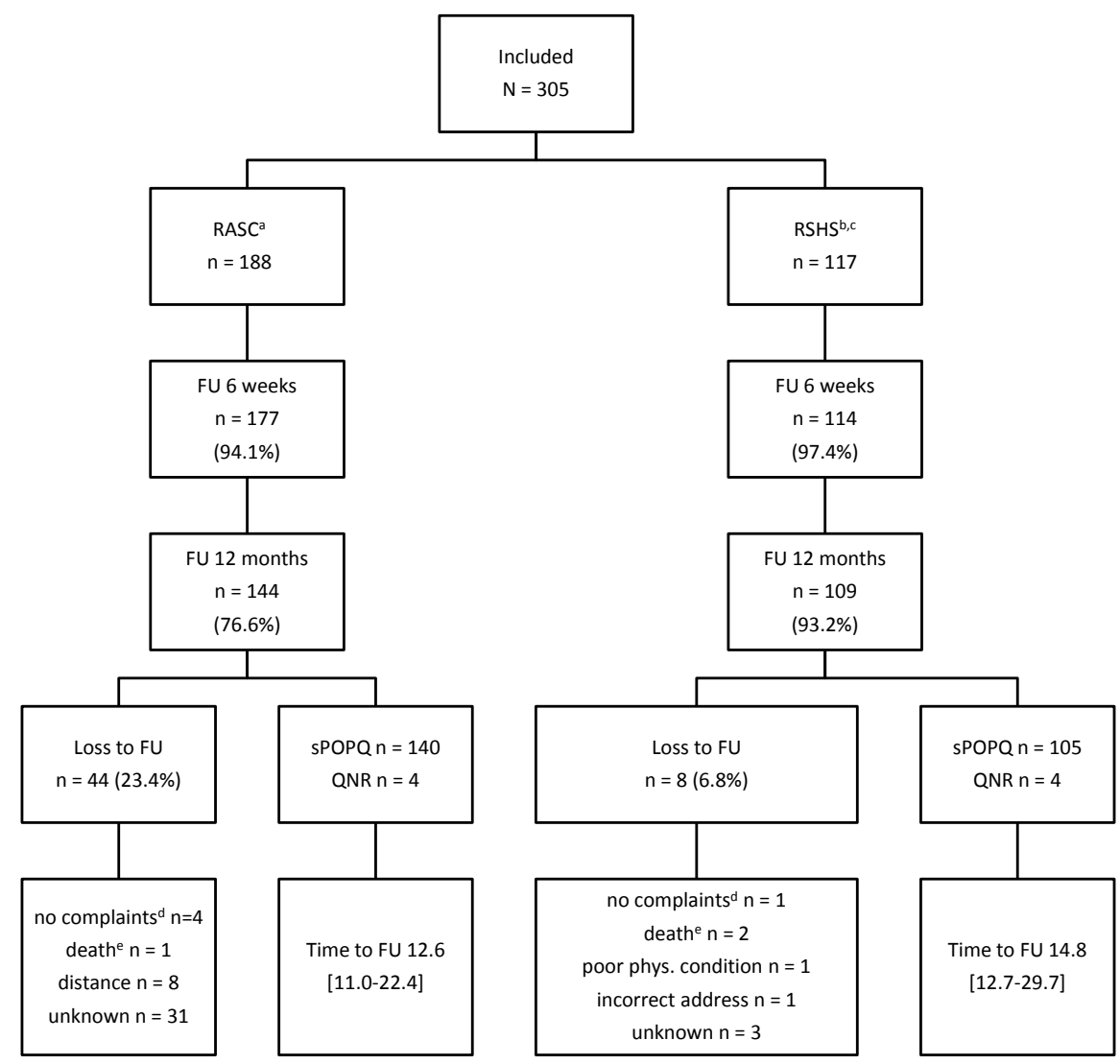

Time to FU is presented as median [IQR]. FU follow up $I Q R$ interquartile range $M o$ months $N$ number $R A S C$ robot-assisted sacrocolpopexy RSHS robot-assisted supracervical hysterectomy with sacrocervicopexy $S P O P Q$ number of patients with a simplified pelvic organ prolapse quantification $Q N R$ questionnaire. ane patient with a history of laparoscopic sacrocolpopexy. ${ }^{\mathrm{b} T}$ Two patients with a history of ventral mesh rectopexy. ${ }^{c}$ One hysteropexy due to adhesions. ${ }^{d P a t i e n t s ~ h a d ~ n o ~ c o m p l a i n t s ~ a n d ~}$ therefore declined consultation. eDue to natural causes. 
Table 1. Baseline characteristics and follow-up data

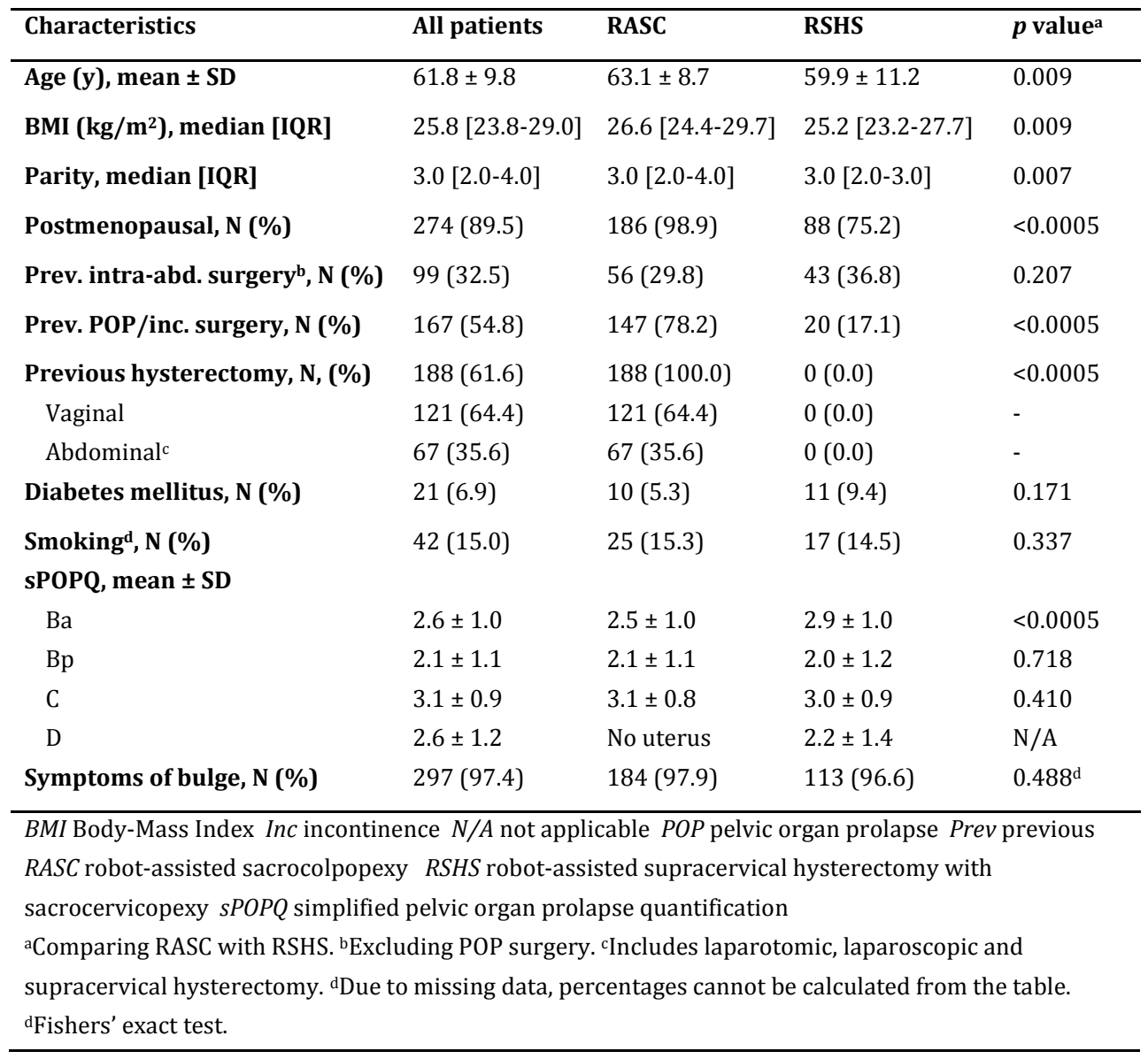

\section{Anatomical results}

For both types of surgery, the mean values for all the SPOPQ anatomical landmarks improved significantly (RASC $p<0.0005$; RSHS $p<0.0005$ ). The apical compartment success rate was $91.4 \%$ for RASC and $99.0 \%$ for RSHS. All compartments were associated with a success rate of $67.1 \%$ for RASC and $64.8 \%$ for RSHS. When solely looking at symptomatic recurrences, however, the success rates increased to $73.6 \%$ and $88.6 \%$, respectively. Complaints of symptoms of bulge diminished significantly after surgery: preoperatively 297 of 305 patients (97.4\%) reported symptoms of bulge; postoperatively 44 of 253 patients $(17.4 \%)$ reported symptoms of bulge $(p<0.0005)$. QoL improved significantly: the mean preoperative PFIQ-7 score was $76.7 \pm 62.3$, and this diminished postoperatively to $13.5 \pm 31.1(p<0.0005)$. Further details of anatomical results are listed in Table 2 . 
Table 2. Recurrences and retreatment

\begin{tabular}{|c|c|c|c|}
\hline & $\begin{array}{l}\text { RASC } \\
\mathrm{N}=188\end{array}$ & $\begin{array}{l}\text { RSHS } \\
\mathrm{N}=117\end{array}$ & $p$ value \\
\hline 6 weeks SPOPQ exam & $\mathrm{n}=177$ & $\mathrm{n}=114$ & \\
\hline Success & $156(88.1)$ & $97(85.1)$ & 0.463 \\
\hline Apical recurrence & $2(1.1)$ & $0(0.0)$ & $0.521^{b}$ \\
\hline Retreatmenta $^{\mathrm{a}}$ & $3(1.7)$ & $0(0.0)$ & $0.282^{\mathrm{b}}$ \\
\hline 12 months SPOPQ exam & $n=140$ & $n=105$ & \\
\hline sPOPQ Ba & 1.4 & 1.4 & $<0.0005^{c}$ \\
\hline sPOPQ Bp & 1.2 & 1.2 & $<0.0005^{c}$ \\
\hline sPOPQ C & 1.1 & 1.0 & $<0.0005^{c}$ \\
\hline sPOPQ D & $\mathrm{N} / \mathrm{A}$ & 1.1 & $<0.0005^{c}$ \\
\hline Success & $94(67.1)$ & $68(64.8)$ & 0.680 \\
\hline No sympt. recurrence. & $103(73.6)$ & $93(88.6)$ & 0.006 \\
\hline Recurrence anterior compart. & $22(15.7)$ & $24(22.9)$ & 0.099 \\
\hline Sympt. recurrence anterior compart. & $17(12.1)$ & $5(4.8)$ & \\
\hline Recurrence post compart. & $6(4.3)$ & $8(7.6)$ & 0.285 \\
\hline Sympt. recurrence posterior compart. & $2(1.4)$ & $4(3.8)$ & \\
\hline Recurrence apical compart. & $1(0.7)$ & $0(0.0)$ & $1.000^{\mathrm{b}}$ \\
\hline Sympt. recurrence apical compart. & $1(0.7)$ & $0(0.0)$ & \\
\hline Recurrence multiple compart. incl. apical & $11(7.9)$ & $1(1.0)$ & 0.012 \\
\hline Sympt. recurrence multiple compart. incl. apical & $11(7.9)$ & $1(1.0)$ & \\
\hline Recurrence multiple compart. not incl. apical & $6(4.3)$ & $4(3.8)$ & $1.000^{\mathrm{b}}$ \\
\hline Sympt. recurrence multiple compart. not incl. apical & $6(4.3)$ & $2(1.9)$ & \\
\hline Retreatments & $\mathrm{n}=144$ & $n=109$ & \\
\hline Prolapse related & $33(22.9)^{\mathrm{d}}$ & $4(3.7)$ & $<0.0005$ \\
\hline Anterior vaginal repair & $12(8.3)^{\mathrm{e}}$ & $2(1.8)$ & \\
\hline Posterior vaginal repair & $6(4.2)$ & - & \\
\hline Anterior and posterior vaginal repair & $12(8.3)^{\mathrm{e}, \mathrm{f}}$ & - & \\
\hline Vaginal pessary & - & $1(0.9)$ & \\
\hline Redo sacrocolpopexy & - & $1(0.9)^{\mathrm{g}}$ & \\
\hline Other & $3^{\mathrm{h}}(2.1)$ & - & \\
\hline Complication related & $3(2.1)$ & $3(2.8)$ & $1.000^{\mathrm{b}}$ \\
\hline Remove (part) mesh & $3^{\mathrm{i}}(2.1)$ & $1(0.9)^{j}$ & \\
\hline ACNES & - & $1(0.9)$ & \\
\hline Incisional hernia & - & $1(0.9)$ & \\
\hline
\end{tabular}


Table 2. Continued

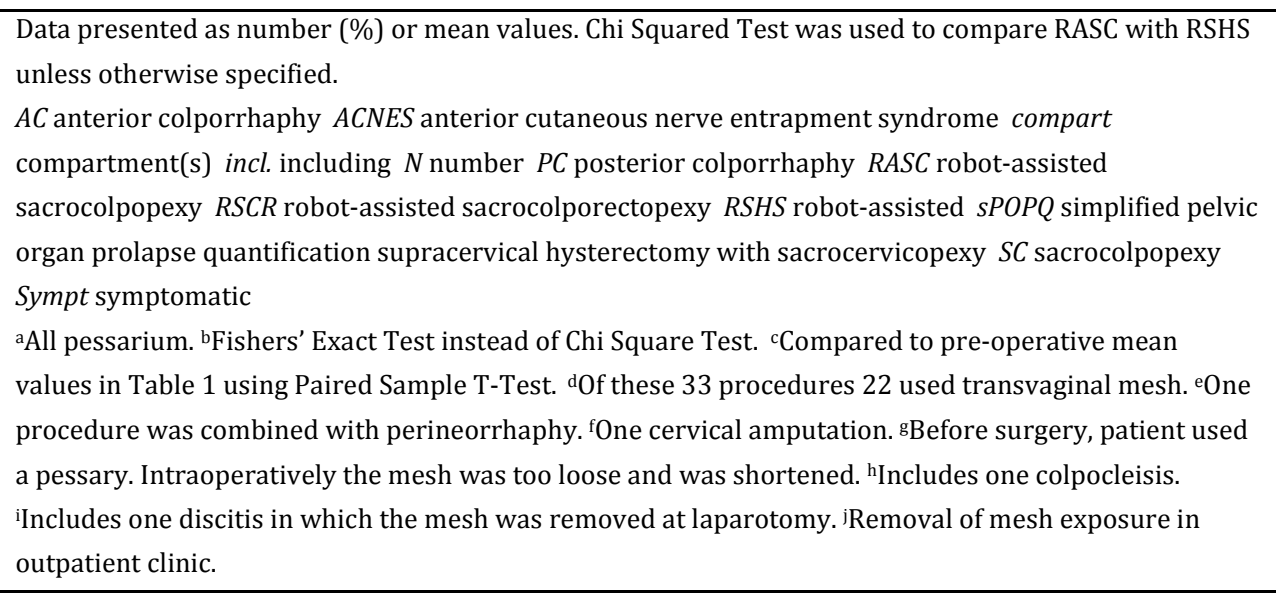

Robot-assisted sacrocolpopexy ( $N=188)$

At 6 weeks after surgery $88.1 \%$ of patients showed no prolapse $(\mathrm{sPOPQ}=1$ for all anatomical landmarks). There were two apical recurrences, both of stage 2 (1.1\%). After 12 months, 94 of 140 examined patients (67.1\%) showed no objective recurrence and 103 patients (73.6\%) had no symptomatic recurrence (Table 2). One hundred and twenty-eight patients had no recurrence in the apical compartment (91.4\%). Of the 12 patients with apical recurrence, more than half were stage 2 [stage $2, n=7$ (5.0\%); stage $3, n=4(2.9 \%)$; stage $4, n=1(0.7 \%)]$. When assessing all three compartments an isolated anterior wall prolapse occurred most frequently in 22 patients $(15.7 \%)$ [sPOPQ stage 2, $n=10(7.1 \%)$; stage $3, n=9(6.4 \%)$; stage $4, n=1(0.7 \%)$; unreported stage at repeat surgery, $n=2(1.4 \%)]$. Assessing recurrences, isolated cystoceles accounted for $47.8 \%$ of recurrences; nine recurrences were asymptomatic (19.6\%). Approximately one-quarter $(22.9 \%)$ of the postoperative patients required a prolapse-related reoperation, mostly consisting of vaginal repair (Table 3). Eight out of 140 patients (5.7\%) reported symptoms of bulge, but had no objective prolapse during physical examination. Compared with RSHS, there was no difference in the percentage of objective success; however, a significant difference was found in the number of patients with a symptomatic recurrence (73.6 versus $88.6 \%$; $p=0.006$ ). In RASC, 184/188 patients (97.9\%) reported preoperative symptoms of bulge versus $26 / 144$ patients $(18.1 \%)$ postoperatively $(p<0.0005)$. PFIQ-7 scores improved significantly: $89.7 \pm 64.1$ preoperatively versus $14.6 \pm 32.3$ postoperatively $(p<0.0005)$. 
Table 3. Intra-operative variables, hospital stay, pain scores and postoperative complications

\begin{tabular}{|c|c|c|c|}
\hline Characteristics & RASC N=188 & RSHS N=117 & $p$ value \\
\hline Intraoperative complications & $10(5.3)$ & $0(0.0)$ & $0.008^{\mathrm{a}}$ \\
\hline Bladder injury & $4(2.1)$ & - & \\
\hline Bladder injury resulting in conversion & $2(1.1)$ & - & \\
\hline Conversion for bleeding & $2(1.1)^{\mathrm{b}}$ & - & \\
\hline Vaginal injury & $1(0.5)$ & - & \\
\hline Ureteric injury & $1(0.5)$ & - & \\
\hline Intraoperative conversions & $8(4.3)$ & $0(0.0)$ & $0.026^{\mathrm{a}}$ \\
\hline Intraoperative complication & $4(2.1)$ & - & \\
\hline Adhesions & $1(0.5)$ & - & \\
\hline Promontory inaccessible & $3(1.6)^{\mathrm{c}}$ & - & \\
\hline Concomitant surgeryd & $15(8.0)$ & $38(32.5)$ & $<0.0005$ \\
\hline TVT & $4(2.1)$ & $11(9.4)$ & 0.004 \\
\hline Adnexal (single/bilateral) & $1(0.5)$ & $10(8.5 \mathrm{~s})$ & $<0.0005^{\mathrm{a}}$ \\
\hline Anterior colporrhaphy & $6(3.2)$ & $13(11.1)$ & 0.005 \\
\hline Posterior colporrhaphy & $3(1.6)$ & $5(4.3)$ & $0.268^{\mathrm{a}}$ \\
\hline Other & $2(1.1)$ & $2(1.7)$ & $0.640^{\mathrm{a}}$ \\
\hline Salpingectomy & $18(9.6)$ & $38(32.5)$ & $<0.0005$ \\
\hline Blood loss in millimeters, median (IQR) & $25(10-50)$ & $50(10-100)$ & 0.007 \\
\hline Total surgery time, mean \pm SD & $145.3 \pm 29.8$ & $183.1 \pm 38.2$ & $<0.0005$ \\
\hline Hospital stay nights, median (IQR) & $1.0(1-2)$ & $2.0(1-3)$ & $<0.0005$ \\
\hline VAS, median (IQR) & $2.0(1-3)$ & $2.5(2-4)$ & 0.305 \\
\hline Early postoperative complications, & $16(8.5)$ & $6(5.1)$ & 0.341 \\
\hline \multicolumn{4}{|l|}{ Clavien-Dindo classification } \\
\hline Grade 1 & $4(2.1)$ & $1(0.9)$ & \\
\hline Grade 2 & $8(4.3)$ & $4(3.4)$ & \\
\hline Grade 3 & $1(0.5)$ & - & \\
\hline Grade 4 & $3(1.6)$ & $1(0.9)$ & \\
\hline Grade 5 & - & - & \\
\hline Late complications & $4 / 144(2.8)$ & $4 / 109(3.7)$ & 0.472 \\
\hline
\end{tabular}

Data presented as number (\%), unless otherwise specified.

$I Q R$ interquartile range $N / A$ not applicable $R A S C$ robot-assisted sacrocolpopexy RSHS robot-assisted supracervical hysterectomy with sacrocervicopexy TVT Tension-free vaginal tape.

aFishers' Exact Test instead of Chi Squared Test (expected count $<5$ ). b ${ }^{\text {b }}$ onversion to laparotomy as first step to stop the bleeding; secondly an anterior colporrhaphy was performed. 'Includes one conversion to sacrospinal fixation with anterior/posterior colporrhaphy. ${ }^{\mathrm{d}}$ More than one concomitant surgery in one patient was possible: scores do not add up. 
Robot-assisted supracervical hysterectomy with sacrocervicopexy $(N=117)$

After 6 weeks, no recurrences were found in the apical compartment. After 1 year, one patient had a recurrent prolapse of the apical compartment (stage 4, 1.0\%; Table 2). A redo-cervicopexy was performed, which revealed a laxity in the mesh; it was shortened, with no recurrence afterwards. Across all compartments, there were 37 recurrences (35.2\%), 12 of which were symptomatic (11.4\%). Twenty-four recurrences were anterior compartment prolapses, mostly at stage $2(\mathrm{n}=18)$ and asymptomatic. In $3.7 \%$ of patients, prolapse-related retreatment was necessary, including the redo-cervicopexy mentioned above. One hundred and thirteen of 117 patients (96.6\%) reported symptoms of bulge prior to surgery versus 18 of 109 patients (16.5\%) after surgery $(p<0.0005)$. A significant improvement in QoL scores was found: $53.7 \pm 52.0$ versus 12.5 $\pm 30.0(p=0.002)$. In one of the postoperative pathology examinations, one patient with endometrial cancer was identified. Further diagnostics showed an endometrial cancer of International Federation of Gynecology and Obstetrics (FIGO) stage IVB.

\section{Secondary outcomes}

Intraoperative complications

In total, ten intraoperative complications (3.3\%) were identified in both groups. All of these complications occurred in the RASC group, which was significantly higher in comparison with the RSHS group (Table 3; 5.3 versus $0.0 \% ; p=0.008$ ). The most common complication was cystotomy (6/10), two of which resulted in conversion. There was one haemorrhage from the presacral venous plexus resulting from the use of a metal retractor for holding the small bowel out of the operative field. There were eight conversions, four as a result of intraoperative complications, one of which was because of excessive adhesions. Three were the result of atypical anatomy of the sacral promontory (prominent vasculature and therefore a high risk of haemorrhage; unidentifiable sacral promontory because of significant presacral fat). Furthermore, in two cases, ventilation problems in steep Trendelenburg position prior to incision occurred: one surgery was abandoned and in the other an open sacrocolpopexy was performed instead.

Intraoperative data

The intraoperative variables are listed in Table 3. Concomitant procedures such as tension-free vaginal tape obturator (TVT-0), salpingo-oophorectomy, and anterior colporrhaphy were significantly performed more frequently in RSHS than RASC [9.4 
versus $2.1 \%(p=0.004) ; 8.5$ versus $0.5 \%(p<0.0005) ; 11.1$ versus $3.2 \%(p=0.005)]$. The duration of surgery was the lowest in the RASC group $(145.3 \pm 29.8$ minutes). The performance of supracervical hysterectomy made the surgery significantly longer (mean difference 38 minutes). Median blood loss was low for both surgeries: 25-50 ml (IQR $10-100 \mathrm{ml})$.

Early postoperative complications

There were 22 (7.2\%) early postoperative complications: 16 (8.5\%) after RASC and six (5.1\%) after RSHS. The majority were minor stage- 1 or -2 complications requiring small interventions. Five complications were severe (Clavien-Dindo classification $\geq 3$; Table 3). One ischaemic cerebrovascular accident (CVA) occurred after RSHS (0.9\%), with full recovery after therapy with anticoagulants. The remaining severe postoperative complications occurred after RASC (2.1\%): one incisional hernia needed surgical correction; one haemorrhagic CVA resulted in subdural haematomas requiring surgery; and one bowel perforation required colostomy and admission to an intensive care unit (ICU).

Late complications

There were four mesh-related complications after RASC (2.1\%): three vaginal mesh exposures and one patient with a vaginal mesh exposure and sacral discitis. Two of these four patients needed complete surgical mesh removal of the mesh $(1.1 \%)$. Four late complications occurred after RSHS (3.4\%): one vaginal mesh exposure, one exposure of a concomitant inserted TVT-0, and two incisional port herniations, with one needing surgical correction. In total, with early postoperative complications included, $1.0 \%$ of patients $(3 / 305)$ were identified with an incisional hernia at a trocar incision site.

\section{Discussion}

\section{Main findings}

The results from this large bi-centre prospective cohort study demonstrate that the robotic approach is an effective and reproducible technique with excellent results associated with the apical compartment (91-99\%). Recurrences were mostly located in the anterior compartment: $15.7 \%$ after RASC (symptomatic $12.1 \%$ ) and $22.9 \%$ after RSHS (symptomatic 4.8\%). Both QoL and the subjective symptoms of bulge improved 
significantly. Intra- and postoperative complications were low. The mean duration of surgery was 145 minutes for RASC and 183 minutes for RSHS.

\section{Strengths and limitations}

Strengths of this study include the size of study group, the prospective design, high recall, and the duration of follow-up. Further strengths were the avoidance of heterogeneity across both the surgery subgroups and the surgical technique used, as well as the experience of the surgeons involved. The main goal of this study was to provide accurate numbers for each procedure, as often these two subtypes of surgery are not clearly differentiated in other studies. The choice of RASC or RSHS was dependent upon the presence of the uterus. As there were differences in baseline characteristics between RASC and RSHS, interpretation of comparisons between the results should therefore be made with caution. This was a limitation of this study.

\section{Interpretation}

The results regarding the apical compartment compare favourably with results from a previously reported systematic review. ${ }^{3}$ The systematic review reported success rates for all compartments from 84 to $100 \%$, which are higher than the success rates of $65-$ $67 \%$ in this study. The systematic review included papers with low numbers of patients, heterogeneous surgeries, different definitions, and variable follow-up periods, and most were retrospective by design. Only one study by Culligan et al. prospectively presented 1-year anatomical data on more than 100 robotic cases $(n=150$; physical exam $n=$ 143). ${ }^{16}$ Although their definition of success was comparable with ours, they reported on a heterogenous group of sacrocolpopexy and sacrocervicopexy. Approximately $80 \%$ of their group required a concomitant supracervical hysterectomy, which affects the surgical variables. They also had a much higher rate of concomitant anti-incontinence surgery $(81 \%)$ than our two groups combined (5\%). Of note, most of their recurrences were seen in the anterior compartment, similar to our findings. When solely looking at symptomatic recurrences occurring for all compartments, we found a 74 and 89\% success rate for RASC and RSHS, respectively. Nygaard et al. performed a large systematic review assessing abdominal sacrocolpopexy, with a follow-up that ranged from 6 months to 3 years, and showed an apical success rate of 78-100\%.17 Success rates in all compartments varied from 58 to $100 \%$, showing that women are at risk for postoperative prolapse in other compartments. 
Historically the treatment of anterior wall prolapse has been problematic, ${ }^{18}$ and as our study illustrates it is similar after both RASC and RSHS. Placing the mesh as distal as possible on the anterior vaginal wall could possibly improve anterior compartment results. There is huge variation in the techniques used by surgeons to anchor the mesh to the anterior vaginal wall, as described previously, which possibly leads to different results. ${ }^{19}$ Wong et al. described 79 women undergoing laparoscopic sacrocolpopexy (LSC), who considered themselves postoperatively cured or improved with no reoperation. ${ }^{20}$ After 3 years, 62\% showed recurrence in the anterior compartment. Furthermore, this highlighted that for every $\mathrm{mm}$ that the mesh was located further from the bladder neck (on Valsalva), the probability of a recurrent cystocele increases by 6$7 \%$. Placing the mesh as close as possible to the bladder neck may improve the recurrence rate, and the robotic system should facilitate this difficult and challenging dissection with the improved freedom of movement and better suturing skills; however, as it might also increase complications rates, further research is necessary to confirm these theories.

Postoperatively a higher percentage of recurrent cystocele was seen after RSHS, which could possibly be explained by the higher SPOPQ stage Ba reported preoperatively, this is associated with a higher risk of recurrence. ${ }^{21}$ Comparisons between the two types of surgeries must be analysed carefully, however, as this study was not set up as a randomized controlled trial. Prendergast et al. ${ }^{22}$ conducted a study where just RSHS was included. The cure rate after 1 year (stage 1, using the standard POPQ assessment) was $72 \%$. We found a success rate of $65 \%$, again, mostly affected by recurrent anterior wall prolapses, many being stage 2 and not symptomatic. When scoring solely symptomatic recurrences, the success percentage rose to $89 \%$ for RSHS. The clinical relevance of asymptomatic prolapse is unclear. Many definitions to describe success after POP repair have been used. ${ }^{23}$ The hymen appears to be an important cut-off point in the occurrence of symptoms, which would be in line with our cut-off point of sPOPQ stage 2 or higher. Repeat surgery, for recurrence, was higher after RASC than after RSHS.

A significantly higher number of intraoperative complications appeared in the RASC group. A history of previous hysterectomy, scar tissue, and adhesions can complicate the RASC procedure, possibly resulting in a higher number of complications. Intraoperative blood loss, duration of hospital stay, and postoperative pain scores were low overall. The duration of surgery time was prolonged when a supracervical hysterectomy was included. Two RCTs on RASC have reported procedure and total surgery time: $227 \pm 47$ 
and $265 \pm 50$ minutes, respectively ( $n=35$, all post-hysterectomy patients); $202.8 \pm 46.1$ and $246.5 \pm 51.3$ minutes, respectively $(n=40$, concomitant hysterectomy $n=25){ }^{6}$ Surgeons were required to have performed at least ten procedures of RASC before participation in the study. The mean surgery time in our study is shorter, probably because of the larger number of patients included and the expertise of the surgeons. Increasing surgical expertise is associated with reduced operative times. ${ }^{24}$

Based on the low percentage of severe early and late postoperative complications, both procedures can be classified as safe. In RSHS, one patient with endometrial cancer was identified postoperatively. Proper preoperative workup should be performed for patients with a uterus. As the US Food and Drug Administration (FDA) suggested in-bag morcellation in their statement in $2014,{ }^{25}$ we started in-bag morcellation to avoid the risk of morcellating a possible malignancy intra-abdominally. Postoperative pain scores were low for both procedures. The duration of hospital stay was significantly shorter for RASC than for RSHS, which could possibly be explained by different postoperative hospital regimes.

Strengths of this study include the size of study group, the prospective design, high recall, and the duration of follow-up. Further strengths were the avoidance of heterogeneity across both the surgery subgroups and the surgical technique used, as well as the experience of the surgeons involved.

This study provides answers to the questions: (1) when I am a competent robotic surgeon, how long on average should it take to perform either a sacrocolpopexy or a sacrocervicopexy; (2) when counselling patients regarding success rates for robotassisted apical prolapse surgery, what are the figures for each subgroup; (3) are each of the robot-assisted surgical procedures for apical prolapse safe for my patients?

\section{Conclusion}

This large prospective cohort study shows that robot-assisted apical repair surgery gives durable anatomical results. Apical success rates were 91 and 99\% for RASC and RSHS, respectively. Postoperative anterior wall recurrences can occur and patients should be counselled accordingly. Both procedures are safe and, when performed regularly, are performed within accessible time ranges. 


\section{References}

1. Akl MN, Long JB, Giles DL, Cornella JL, Pettit PD, Chen AH, et al. Robotic-assisted sacrocolpopexy: technique and learning curve. Surg Endosc 2009;23:2390-4.

2. Tarr ME, Brancato SJ, Cunkelman JA, Polcari A, Nutter B, Kenton K. Comparison of postural ergonomics between laparoscopic and robotic sacrocolpopexy: a pilot study. J Minim Invasive Gynecol 2015;22:234-8.

3. Serati M, Bogani G, Sorice P, Braga A, Torella M, Salvatore S, et al. Robot-assisted sacrocolpopexy for pelvic organ prolapse: a systematic review and meta-analysis of comparative studies. Eur Urol 2014;66:303-18.

4. Anglim B, O Sullivan OE, O'Reilly BA. How do patients and surgeons decide on uterine preservation or hysterectomy in apical prolapse? Int Urogynecol J 2018;29:1075-9.

5. Maher C, Feiner B, Baessler K, Christmann-Schmid C, Haya N, Brown J. Surgery for women with apical vaginal prolapse. Cochrane Database Syst Rev 2016;(10):CD012376.

6. Paraiso MF, Jelovsek JE, Frick A, Chen CC, Barber MD. Laparoscopic compared with robotic sacrocolpopexy for vaginal prolapse: a randomized controlled trial. Obstet Gynecol 2011;118:1005-13.

7. Anger JT, Mueller ER, Tarnay C, Smith B, Stroupe K, Rosenman A, et al. Robotic compared with laparoscopic sacrocolpopexy: a randomized controlled trial. Obstet Gynecol 2014;123:5-12.

8. Collins S, Tulikangas P. Randomized trials in robotic surgery: a practical impossibility? Int Urogynecol J 2010;21:1045-7.

9. Swift S, Morris S, McKinnie V, Freeman R, Petri E, Scotti RJ, et al. Validation of a simplified technique for using the POPQ pelvic organ prolapse classification system. Int Urogynecol J Pelvic Floor Dysfunct 2006;17:615-20.

10. Parekh M, Swift S, Lemos N, Iskander M, Freeman B, Arunkalaivanan AS, et al. Multicenter interexaminer agreement trial for the validation of simplified POPQ system. Int Urogynecol J 2011;22:645-50.

11. Utomo E, Blok BF, Steensma AB, Korfage IJ. Validation of the Pelvic Floor Distress Inventory (PFDI-20) and Pelvic Floor Impact Questionnaire (PFIQ-7) in a Dutch population. Int Urogynecol J 2014; 25:531-44.

12. Rosenthal R, Hoffmann H, Clavien PA, Bucher HC, Dell-Kuster S. Definition and classification of intraoperative complications (CLASSIC): Delphi study and pilot evaluation. World J Surg 2015;39:1663-71.

13. Dindo D, Demartines N, Clavien PA. Classification of surgical complications: a new proposal with evaluation in a cohort of 6336 patients and results of a survey. Ann Surg 2004;240:205-13.

14. Clifton MM, Pizarro-Berdichevsky J, Goldman HB. Robotic female pelvic floor reconstruction: a review. Urology 2016;91:33-40.

15. Rimbach S, Schempershofe M. In-bag morcellation as a routine for laparoscopic hysterectomy. Biomed Res Int 2017; article ID 6701916:1-6.

16. Culligan PJ, Gurshumov E, Lewis C, Priestley JL, Komar J, Shah N, et al. Subjective and objective results 1 year after robotic sacrocolpopexy using a lightweight Y-mesh. Int Urogynecol J 2014;25:731-5. 
17. Nygaard IE, McCreery R, Brubaker L, Connolly A, Cundiff G, Weber AM, et al. Abdominal sacrocolpopexy: a comprehensive review. Obstet Gynecol 2004;104:805-23.

18. Lensen EJ, Withagen MI, Kluivers KB, Milani AL, Vierhout ME. Surgical treatment of pelvic organ prolapse: a historical review with emphasis on the anterior compartment. Int Urogynecol J 2013;24:1593-602.

19. O'Sullivan OE, Matthews CA, O'Reilly BA. Sacrocolpopexy: is there a consistent surgical technique? Int Urogynecol J 2016;27:747-50.

20. Wong V, Guzman RR, Shek KL, Chou D, Moore KH, Dietz HP. Laparoscopic sacrocolpopexy: how low does the mesh go? Ultrasound Obstet Gynecol 2017;49:404-8.

21. Aslam MF, Osmundsen B, Edwards SR, Matthews C, Gregory WT. Preoperative prolapse stage as predictor of failure of sacrocolpopexy. Female Pelvic Med Reconstr Surg 2016;22:156-60.

22. Prendergast E, Silver H, Johnson LL, Simon M, Feinglass J, Kielb S, et al. Anatomic outcomes of robotic assisted supracervical hysterectomy and concurrent sacrocolpopexy at a tertiary care institution at initial adaptation of the procedure. Female Pelvic Med Reconstr Surg 2016;22:29-32.

23. Barber MD, Brubaker L, Nygaard I, Wheeler TL II, Schaffer J, Chen Z, et al. Defining success after surgery for pelvic organ prolapse. Obstet Gynecol 2009;114:600-9.

24. Lenihan JP Jr, Kovanda C, Seshadri-Kreaden U. What is the learning curve for robotic assisted gynecologic surgery? J Minim Invasive Gynecol 2008;15:589-94.

25. US Food and Drug Administration. Laparoscopic uterine power morcellation in hysterectomy and myomectomy: FDA safety communication. 2014 http://www.fda.gov/medicaldevices/safety/ alertsandnotices/ucm393576.htm. Accessed 20 April 2019. 




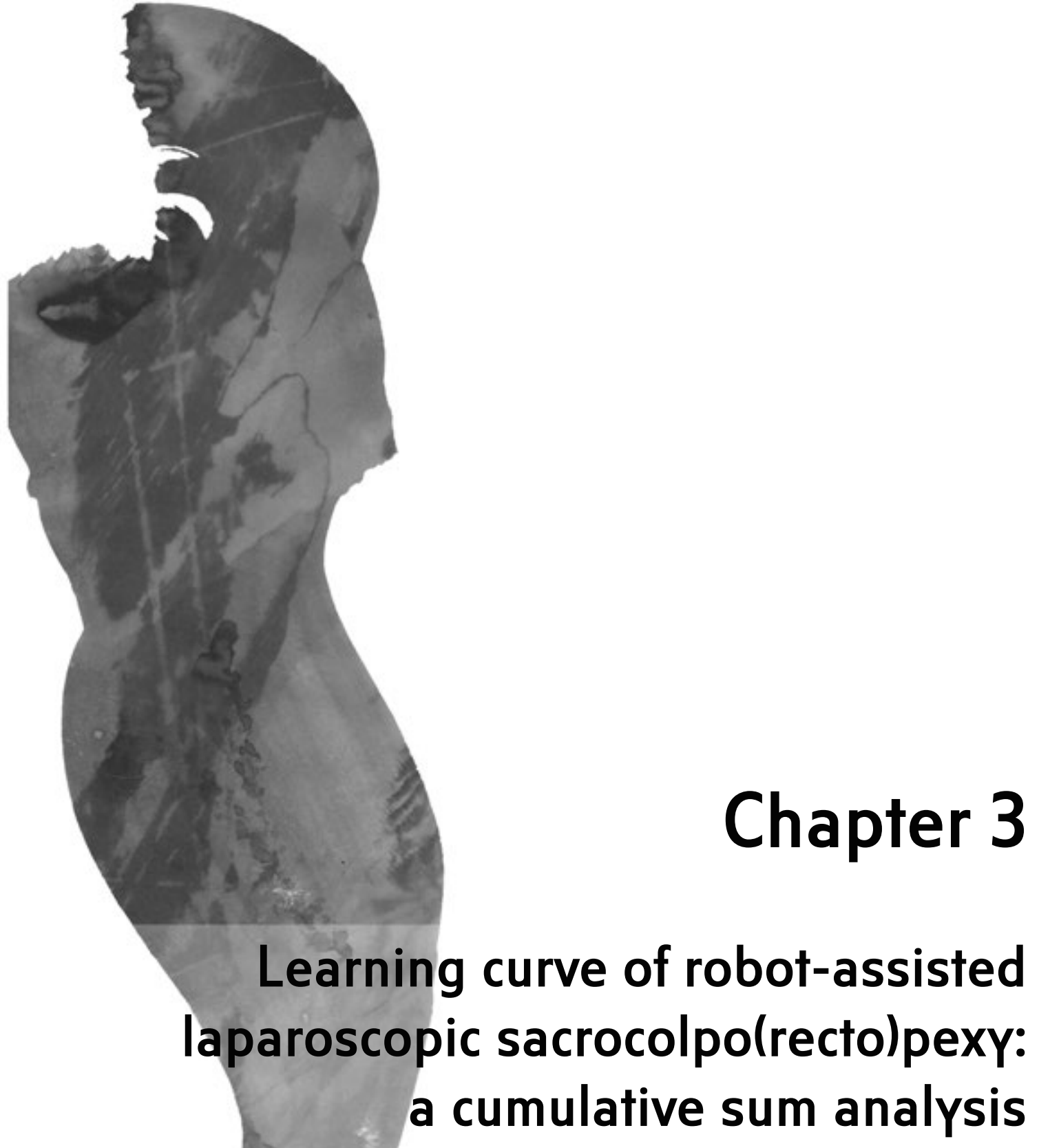

Femke van Zanten, Steven E. Schraffordt Koops, Pieternel C.M. Pasker-de Jong. Egbert Lenters Henk W.R. Schreuder

American Journal of Obstetrics and Gynecology 2019;221:483.e1-11 


\section{Abstract}

Background: Determination of the learning curve of new techniques is essential to improve safety and efficiency. Limited information is available regarding learning curves in robot-assisted laparoscopic pelvic floor surgery.

Objective: The purpose of this study was to assess the learning curve in robot-assisted laparoscopic pelvic floor surgery.

Study Design: We conducted a prospective cohort study. Consecutive patients undergoing robot-assisted laparoscopic sacrocolpopexy or sacrocolporectopexy were included $(\mathrm{N}=372)$. Patients were treated in a teaching hospital with a tertiary referral function for gynaecological/multicompartment prolapse. Procedures were performed by two experienced conventional laparoscopic surgeons (surgeons A and B). Baseline demographics were scored per groups of 25 consecutive patients. The primary outcome was the determination of proficiency, which was based on intraoperative complications. Cumulative sum control chart analysis allowed us to detect small shifts in a surgeon's performance. Proficiency was obtained when the first acceptable boundary line of cumulative sum control chart analysis was crossed. Secondary outcomes that were examined were shortening and/or stabilization of surgery time (measured with the use of cumulative sum control chart analysis and the moving average method).

Results: Surgeon A performed 242 surgeries; surgeon B 137 ( $n=7$ surgeries were performed by both surgeons). Intraoperative complications occurred in $1.9 \%$ of the procedures. The learning curve never fell below the unacceptable failure limits and stabilized after 23/41 cases. Proficiency was obtained after 78 cases for both surgeons. Surgery time decreased after 24-29 cases in robot-assisted sacrocolpopexy (no distinct pattern for robot-assisted sacrocolporectopexy). Limitations were the inclusion of 2 interventions and concomitant procedures, which limited homogeneity. Furthermore, analyses treated all complications in cumulative sum as equal weight, although there are differences in the clinical relevance of complications.

Conclusions: After 78 cases proficiency was obtained. After 24-29 cases, surgery time stabilized for robot-assisted sacrocolpopexy. In this age of rapidly changing surgical techniques, it can be difficult to determine the learning curve of each procedure. Cumulative sum control chart analysis can assist with this determination and prove to be a valuable tool. Training programs could be individualized to improve both surgical performance and patient benefits. 


\section{Introduction}

The arrival of robot-assisted laparoscopic surgery strongly affected surgical approaches to treat apical prolapse. ${ }^{1}$ This has also impacted training in gynaecologic surgery, by broadening the surgical education that is required. ${ }^{2}$ With the rising implementation of new techniques, standardization of self-teaching programs and quality-control measurements are very much needed to assess safety and optimize patient outcomes. Evaluation of learning curves allows for more accurate detection of potential pitfalls and could improve surgical training. Cumulative sum control chart (CUSUM) analysis has been shown to evaluate surgical performance efficiently by detecting small shifts in the parameters of a process, ${ }^{3,4}$ which results in the visualizing of trends that would not be detectable with other techniques. CUSUM analysis can be used as self-monitoring tool or to compare results with numbers from the literature. The analysis can mark phases in which complications arise, thereby warning the surgeon to change the training program or add additional training. A limitation of the CUSUM procedure is that it may signal not a change in surgical failure rate, but in the referral pattern. An increased proportion of high-risk patients could be reason for a rise in complication rate, rather than a change in surgical performance. ${ }^{3}$ This must be examined before training is adjusted.

The objective of this study was to use CUSUM analysis to examine the learning curve of robot-assisted laparoscopic sacrocolpopexy (RASC) for experienced laparoscopic surgeons. This could help determine the best format of structured training programs for laparoscopic surgeons to incorporate robot-assisted surgery safely and efficiently.

\section{Material and Methods}

All patients were treated in 1 large teaching hospital with a tertiary referral function for patients with gynaecological prolapse. Consecutive patients undergoing RASC or robotassisted sacrocolporectopexy (RSCR) in case of multicompartment prolapse were included. If the uterus was present, a subtotal hysterectomy was performed concomitantly. Surgical techniques and materials have been described in detail previously.5,6 All procedures were performed with the aid of the da Vinci Si-HD system (Intuitive Surgical, Inc, Sunnyvale, CA). Pneumoperitoneum was created through a Veress needle or Hasson open entry. Placement of two 12-mm and three 8-mm robotic trocars followed (intra-abdominal pressure $12 \mathrm{~mm} \mathrm{Hg}$ ). Patients were placed in the lithotomy and Trendelenburg positions. The peritoneum was incised to reveal the 
promontory and to create an anterior vesicovaginal and posterior rectovaginal space. The mesh (Prolene; weight 80-85 g/m2; Ethicon Inc, Johnson \& Johnson, Hamburg, Germany) was sutured distally with nonabsorbable sutures to the posterior and anterior vaginal wall and vaginal cuff or cervix. The 2 meshes were configured intracorporeally to a Y-shape. The posterior mesh was attached proximally to the sacral promontory with titanium tacks (Autosuture Protack 5 mm; Covidien, Minneapolis, MN). In case of RSCR, the colorectal surgeon started the procedure by performing a ventral mesh rectopexy according to the procedure described by D'Hoore and Penninckx. ${ }^{6}$ In this case, no separate second mesh was placed directly on the posterior vaginal wall: the ventral rectopexy mesh was sutured on the anterior side to the posterior vaginal wall. The peritoneum was closed over the graft with a V-Loc suture (Covidien).

Surgical details of the procedures from the start of the use of robot-assistance until 4 years later were evaluated prospectively. Surgeries were performed by 2 gynecologists: 'surgeon A' and 'surgeon B'. Surgeon A had 17 years of conventional laparoscopic experience and surgeon B 21 years of experience at the starting point. They had each performed approximately 300 laparoscopic sacrocolpopexy procedures. Before using robot assistance, surgeons and their surgical team followed a robotic learning course on cadavers. They had no previous robotic experience. The first 2 robotic cases that were performed were supervised by an urogynecologist who was experienced in RASC. No other robot-assisted surgeries that could have influenced the learning curve were performed by the surgeons in the study period.

The primary outcome measure was the determination of proficiency. The determination of proficiency was based on intraoperative complications, crossing $\geq 1$ acceptable boundary lines of the CUSUM analysis. A secondary outcome was stabilization of surgery time to a steady state.

CUSUM analysis was performed to detect differences in the surgeon's performance and to determine proficiency. ${ }^{4}$ Results were put into a graph, in which the X-axis represented the number of procedures and the $Y$-axis represented the cumulative sum of success and failure. With each success, the graph will rise by 's' and with each failure, the graph will fall by ' $1-s$ '. ' $s$ ' is dependent on predetermined acceptable and unacceptable failure rates (Appendix). Acceptable failure rate was set at 4.4\%, which was based on prospective or randomized controlled trials that described RASC and intraoperative complications $(n=14 / 321) .7,8,9,10,11$ Unacceptable failure rate was set at 2 times the acceptable failure 
rate $(8.8 \%)$. When there were no intraoperative complications, the CUSUM graph increased by $s=0.064$ (Appendix). When a complication arose, the graph fell by 0.936 (1s). Boundary lines were calculated to determine whether the surgical performance was acceptable $\left(\mathrm{H}_{1}\right)$ or unacceptable $\left(\mathrm{H}_{-1}\right)^{4,12}$ Crossing more than one boundary line was also possible $\left(\mathrm{H}_{2} / \mathrm{H}_{-2}, \mathrm{H}_{3} / \mathrm{H}_{-3}\right.$, etc), gaining either more skills $\left(\mathrm{H}_{2} / \mathrm{H}_{3}\right)$ or falling further behind $\left(\mathrm{H}_{-2} / \mathrm{H}_{-3}\right)$. With the aid of these multiple boundary lines, the CUSUM graph can also alert surgeons when they first perform acceptably, but then, for some reason, their performance rate decreases. The probability of falsely stating that the surgeon's performance is 'acceptable' or 'unacceptable' is called a type 1 error $(\alpha)$ and type 2 error $(\beta)$. In this study $10 \%$ type 1 and 2 errors were considered acceptable. When performing in the acceptable zone, a surgeon's performance is considered to be significantly better than the acceptable rate, with a false positive rate of $\alpha$. Proficiency was obtained when the graph crossed $\mathrm{H}_{1}$. Proficiency could be lost by crossing 2 (or more) unacceptable boundary lines. When the graph maintains between $\mathrm{H}_{1}$ and $\mathrm{H}-1$, (ie. circles round the 0 line) performance does not improve or deteriorate significantly, and neither nullhypothesis is rejected.

CUSUM analysis was also performed to detect differences in total surgery time. CUSUM analysis was used as a self-assessment tool. The mean surgery time per surgeon was calculated. This value was used as a reference value. When a surgery took more or less time than the mean surgery time, the graph would rise or fall with the absolute difference. Robotic setup time, console time and total surgery time were also analyzed with the use of a moving average method (MOA). In MOA, big fluctuations are filtered out. The mean duration of the first ten surgery times is point 1 , mean duration of surgery $2-11$ is point 2 , etc.

The procedures were scored sequentially based on operation date and time. Data were divided into groups of 25 procedures to look for differences in patients' risk profile. Intraoperative complications were defined as any deviation from the ideal intraoperative course between incision and closure, which included conversions as a result of genitourinary, bowel or vascular injury. Intraoperative complications that were detected postoperatively were scored as well. Because the main objective is surgeon's performance, conversions because of adhesions, anesthetics or malfunction of the robot were excluded. The Satava Classification system was used to score intraoperative complications. ${ }^{13}$ 
Time to set up the robot, total time the surgeon was seated in the console to perform the surgery, and total time from first incision until last suture was defined as 'robot docking time', 'console time' and 'total surgery time' respectively. Times were scored separately for RASC and RSCR. The simplified Pelvic Organ Prolapse Quantification (sPOPQ) 14 examination was used for the determination of stage of prolapse. As result of recent evidence on the prevention of ovarian cancer, in consultation with the patient, a salpingectomy was performed simultaneously at the end of this study. ${ }^{15}$ Because of new national and Food and Drug Administration guidelines, in-bag morcellation was performed starting from 2015 to prevent spill in case a sarcoma would be present. ${ }^{16,17}$ The start of in-bag morcellation and consequent performance of concomitant salpingectomy is marked in the figures.

This study was judged as an exempt study by the National Central Committee on Research Involving Human subjects (CCMO). Statistical analysis was performed with SPSS statistics software (version 22.0; IBM, Armonk, NY) Data were presented as number and percentage for categoric data, mean \pm standard deviation, or median and range for continuous data. The Independent $T$-Test and 1-way analyses of variance, Mann-Whitney $U$ Test, and Kruskal Wallis test were used to test shifts in normally distributed and nonnormally distributed continuous values, respectively. Chi squared test and Fisher's Exact Test were used for categoric data, as appropriate. Post hoc tests were used in case of significance. All tests were considered significant at .05 level.

\section{Results}

In total 372 surgeries were performed. Surgeon A performed 242 surgeries [RASC, 129 (53.3\%); RSCR, 113 (46.7\%)] and Surgeon B 137 surgeries [RASC, 62 (45.3\%); RSCR, 75 (54.7\%)]. Seven procedures were performed by both surgeons. Baseline characteristics and surgical details are shown in Table 1. Concomitant anterior colporrhaphy was performed more often by surgeon B than surgeon A.

Intraoperative complications occurred in $1.9 \%$ of patients $(7 / 372$; Table 2$)$. There were 2 conversions because of adhesions without visceral damage and 2 ventilation-related problems; all of which were not considered to be a complication. There were no conversions because of robotic system failure. Figure 1 shows the CUSUM analysis of the intraoperative complications for both surgeons. Boundary lines were calculated (appendix A). After 2 complications at an early stage, a steadily climbing line was seen 
for surgeon A after 23 cases and for surgeon B after 41 cases. Proficiency was obtained for both surgeons after 78 cases. A second trend for surgeon A was seen: after 172 complication free cases, new complications arose without falling below 2 unacceptable performance lines. The last 3 complications of surgeon A occurred in case numbers 175200. Table 3 shows the baseline characteristics per 25 surgeries, forming 10 groups. As a result of the addition of salpingectomy, the number of concomitant surgeries in case numbers 175-200 for surgeon A were significantly different. Furthermore, more RSCRs were performed, with 3 patients receiving re-rectopexy. Looking at all groups of 25 procedures for surgeon A, there was no significant difference in patients' demographics, besides age and concomitant surgery. For surgeon $B$, there was a significant difference between early and late groups of 25 patients regarding the performance of RSCR and salpingectomy. In-bag morcellation was used starting from procedure number 218 of 242 (RASC, 123/129; RSCR, 96/113) for surgeon A and 126/137 (RASC, 60/62; RSCR, $67 / 75$ ) for surgeon B. Describing changes in patients' profile and surgical procedure aids in understanding the CUSUM analysis.

Table 1. Baseline characteristics and surgical details

\begin{tabular}{|c|c|c|c|c|}
\hline & $\begin{array}{l}\text { Total } \\
\mathrm{N}=372^{\mathrm{a}}\end{array}$ & $\begin{array}{l}\text { Surgeon } A \\
n=242\end{array}$ & $\begin{array}{l}\text { Surgeon B } \\
n=137\end{array}$ & $p$ value $^{b}$ \\
\hline Age in years & $60.5 \pm 11.3$ & $60.2 \pm 11.3$ & $61.1 \pm 11.3$ & 0.424 \\
\hline BMI & $26.4 \pm 3.9$ & $26.3 \pm 3.8$ & $26.6 \pm 4.2$ & 0.572 \\
\hline Parity & $3.0(0-11)$ & $3.0(0-6)$ & $3.0(0-11)$ & 0.364 \\
\hline Postmenopausal & $296(79.6)$ & $190(78.5)$ & 112 (81.8) & 0.299 \\
\hline Diabetes mellitus & $30(8.1)$ & $17(7.0)$ & $13(9.5)$ & 0.393 \\
\hline Active smoker & $67(18.0)$ & $45(18.6)$ & $23(16.8)$ & 0.787 \\
\hline ASA grading & & & & 0.854 \\
\hline ASA 1 & $135(36.3)$ & $89(36.8)$ & $49(35.8)$ & \\
\hline ASA 2 & $200(53.8)$ & $130(53.7)$ & $74(54.0)$ & \\
\hline ASA 3 & $19(5.1)$ & $11(4.5)$ & $8(5.8)$ & \\
\hline Not reported & $18(4.8)$ & $12(5.0)$ & $6(4.4)$ & \\
\hline Previous POP/inc & & & & 0.836 \\
\hline \multicolumn{5}{|l|}{ surgeryc } \\
\hline None & $210(56.5)$ & $138(57.0)$ & 78 (56.9) & \\
\hline 1 & $123(33.1)$ & $79(32.6)$ & 45 (32.8) & \\
\hline 2 & $31(8.3)$ & 21 (8.7) & $10(7.3)$ & \\
\hline$\geq 3$ & $8(2.1)$ & $4(1.7)$ & $4(2.9)$ & \\
\hline Other abd. surgery ${ }^{d}$ & $181(48.7)$ & $117(48.3)$ & $66(48.2)$ & 0.974 \\
\hline
\end{tabular}


Table 1. Continued

\begin{tabular}{|c|c|c|c|c|}
\hline & $\begin{array}{l}\text { Total } \\
\mathrm{N}=372^{\mathrm{a}}\end{array}$ & $\begin{array}{l}\text { Surgeon A } \\
\mathrm{n}=242\end{array}$ & $\begin{array}{l}\text { Surgeon } B \\
n=137\end{array}$ & $p$ value $^{b}$ \\
\hline \multicolumn{5}{|l|}{ SPOPQ } \\
\hline $\mathrm{Ba}$ & $3.0(1-4)$ & $3.0(1-4)$ & $3.0(1-4)$ & 0.336 \\
\hline $\mathrm{Bp}$ & $2.0(1-4)$ & $2.0(1-4)$ & $2.0(1-4)$ & 0.878 \\
\hline $\mathrm{C}$ & $3.0(1-4)$ & $3.0(1-4)$ & $3.0(1-4)$ & $0.004^{\mathrm{e}}$ \\
\hline $\mathrm{D}$ & $2.0(1-4)$ & $2.0(1-4)$ & $2.0(1-4)$ & 0.764 \\
\hline Type of surgery & & & & 0.132 \\
\hline RASC & $184(49.5)^{\mathrm{a}}$ & $129(53.3)$ & $62(45.2)$ & \\
\hline RSCR & $188(50.5)$ & 113 (46.7) & 75 (54.7) & \\
\hline \multicolumn{5}{|c|}{ Concomitant surgery } \\
\hline $\begin{array}{l}\text { Subtotal } \\
\text { hysterectomy }\end{array}$ & $228(61.3)$ & $147(60.7)$ & $81(59.1)$ & 0.827 \\
\hline TVT & $19(5.1)$ & $12(5.0)$ & $8(5.8)$ & 0.812 \\
\hline $\mathrm{AC}$ & $22(5.9)$ & $6(2.5)$ & $16(11.7)$ & $<0.0005^{\mathrm{e}}$ \\
\hline $\mathrm{PC}$ & $5(1.3)$ & $3(1.2)$ & $2(1.5)$ & $0.595^{\mathrm{f}}$ \\
\hline Oophorectomy & $19(5.1)$ & $16(6.6)$ & $3(2.2)$ & 0.058 \\
\hline Salpingectomy & $122(32.8)$ & $88(36.4)$ & $36(26.3)$ & $0.044^{\mathrm{e}}$ \\
\hline Other & $11(3.0)$ & $6(2.5)$ & $5(3.6)$ & $0.536^{f}$ \\
\hline
\end{tabular}

Data presented as mean \pm SD, median and range, or N (\%). Pearson's Chi Squared Test, Independent samples T-test, Mann-Whitney U test were used for categorical data, mean values and median values respectively, unless otherwise specified.

$A b d$ abdominal $A C$ anterior colporrhaphy $A S A$ American society of anesthesiologists $B M I$ body-mass index Inc. incontinence $N$ number $P C$ posterior colporrhaphy $P O P$ pelvic organ prolapse $R A S C$ robotassisted sacrocolpopexy $R S C R$ robot-assisted sacrocolporectopexy $S D$ standard deviation $s P O P Q$ simplified pelvic organ prolapse quantification TVT Tension-free vaginal tape aSeven surgeries performed by both surgeon A and surgeon B: scores do not add up. bomparing Surgeon A with Surgeon B. cIn case two or more POP/inc. procedure were combined during one surgery, this was counted as one POP/inc. surgery. ${ }^{\mathrm{d} A b d o m i n a l ~ s u r g e r y ~ i n c l u d e d ~ i n ~ p r e v i o u s ~ P O P / i n c ~}$ surgery are not included. eStatistically significant. fFisher's exact test ( $>20 \%$ expected count $<5$ ).

Table 2. Intraoperative complications (Satava Classification) ${ }^{5}$

\begin{tabular}{clllll}
\hline & Grade 1 & Grade 2 & Grade 3 & Total & Conversion \\
\hline Surgeon A & 0 & $5^{\mathrm{a}}$ & 0 & $5(2.1 \%)$ & 0 \\
Surgeon B & 0 & $1^{\mathrm{b}}$ & $1^{\mathrm{c}}$ & $2(1.5 \%)$ & 1 \\
Total & 0 & 6 & 1 & $7(1.9 \%)$ & 1 \\
\hline
\end{tabular}

Grade I: incidents without consequences Grade II: incidents repaired intraoperatively Grade III: incidents requiring reoperation.

aBladder lesion $(\mathrm{N}=2)$, bowel serosa lesion $(\mathrm{N}=2)$, vaginotomy $(\mathrm{N}=1)$. ${ }^{\mathrm{b} B l a d d e r}$ lesion. ${ }^{\mathrm{c}}$ Intra-abdominal bleeding. 
Figure 1. CUSUM analysis of intraoperative complications per surgeon.

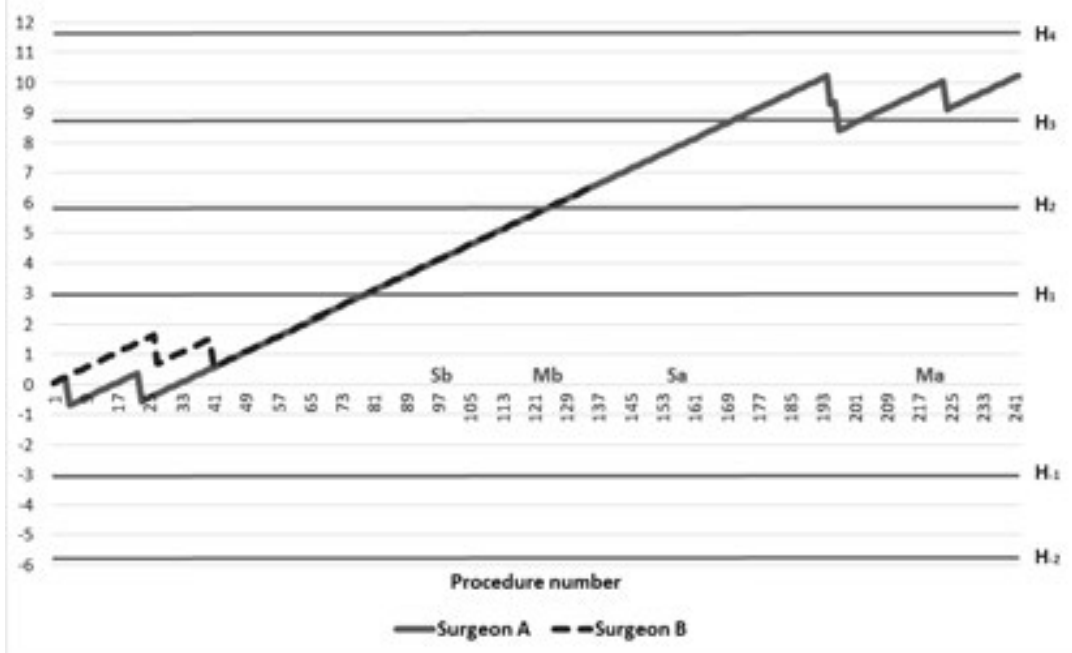

X-axis: number of procedures performed. Y-axis: cumulative sum of succes and failure of the surgeon. Consecutive line: surgeon A. Dotted line: surgeon B. Ma start of endobag morcellation surgeon A $M b$ start of endobag morcellation surgeon B $S a$ start consistent salpingectomy surgeon A $S b$ start consistent salpingectomy surgeon B

Cumulative sum (CUSUM) analysis for intraoperative complications. CUSUM analysis is based on acceptable performance rate of $4.4 \%$ and unacceptable performance rate of $8.8 \%$. In case of a complication the graph falls with 0.936. In case of no complication the graph rises with 0.064 (Appendix A). The horizontal lines represent the upper and lower control limits: the boundary lines. Unacceptable perfomace is achieved when the graph falls below $\mathrm{H}_{-1}$. Proficiency is obtained when the graph crosses $\mathrm{H}_{1}$. The surgeons can cross a second or third boundary line $\left(\mathrm{H}_{2}, \mathrm{H}_{3}\right)$, indicating even better performance. Proficiency can be lost by a fall in the graph and crossing two or more boundary lines. The CUSUM analysis alerts at this point that measurements should be taken to improve performance again. There is an upward slope after 23-41 cases. Proficiency is obtained after 78 cases for both surgeons. For surgeon A, a second trend in complications is seen after 172 complication free cases. The graph never falls in the unacceptable performance zone. 


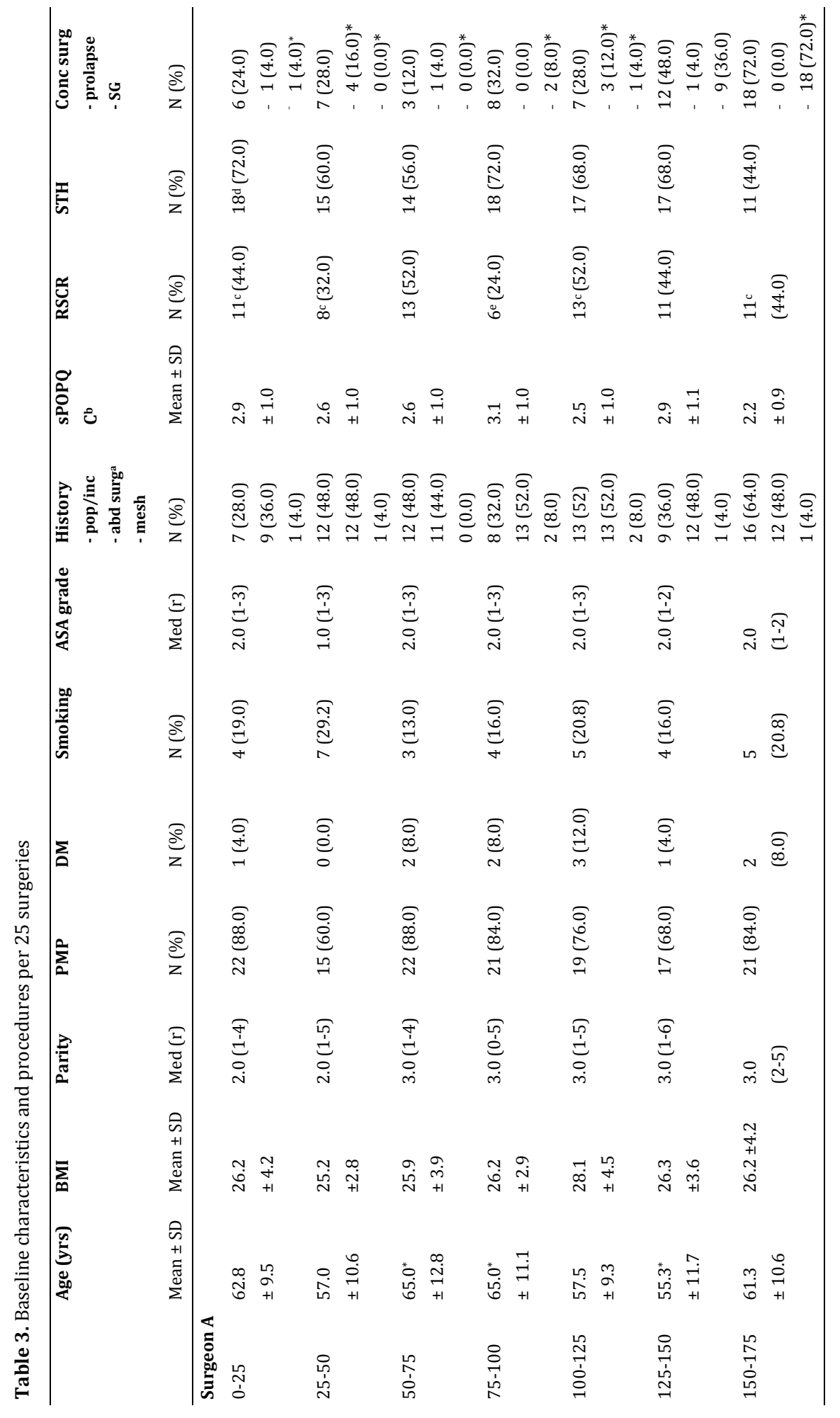




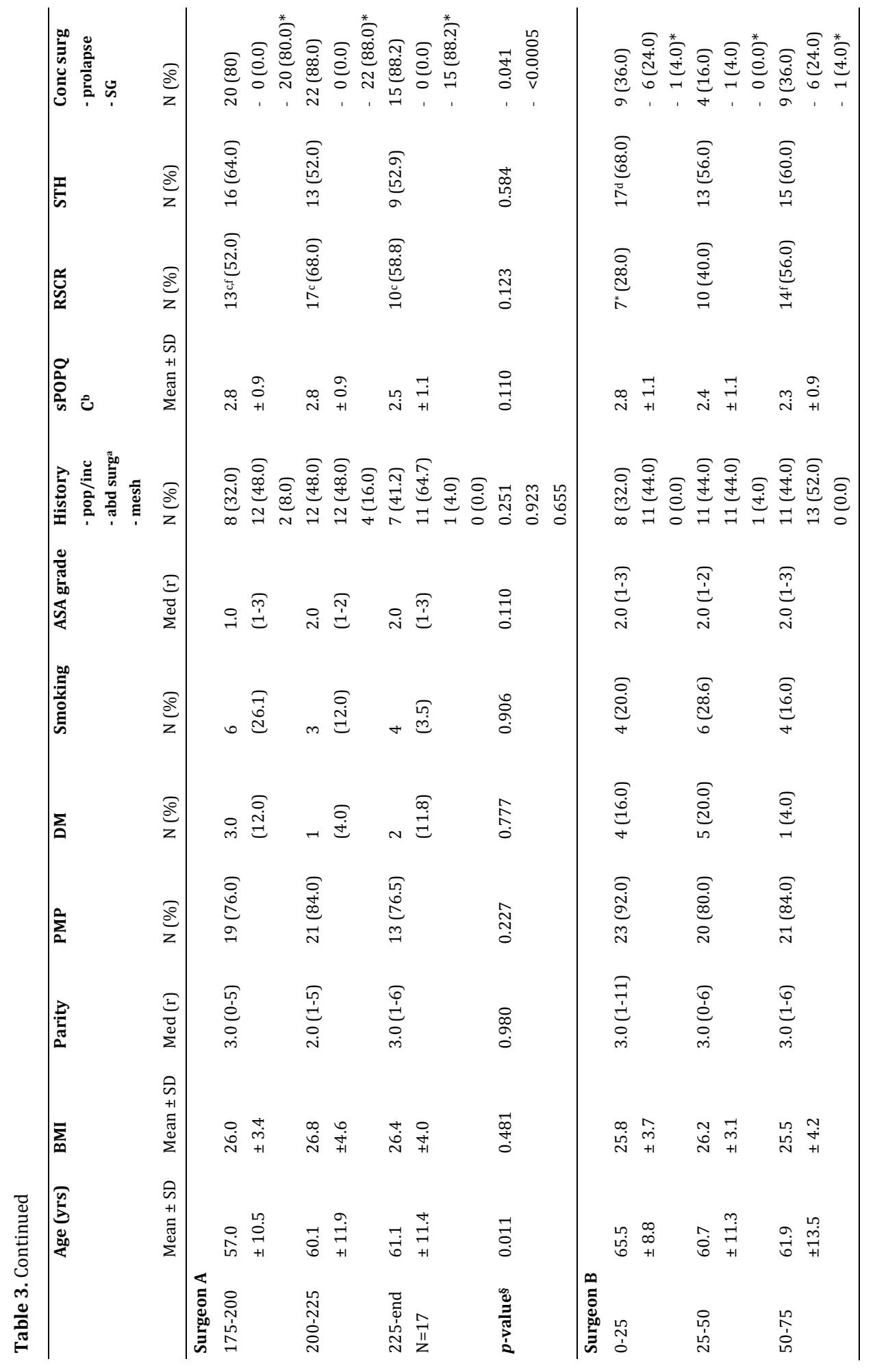




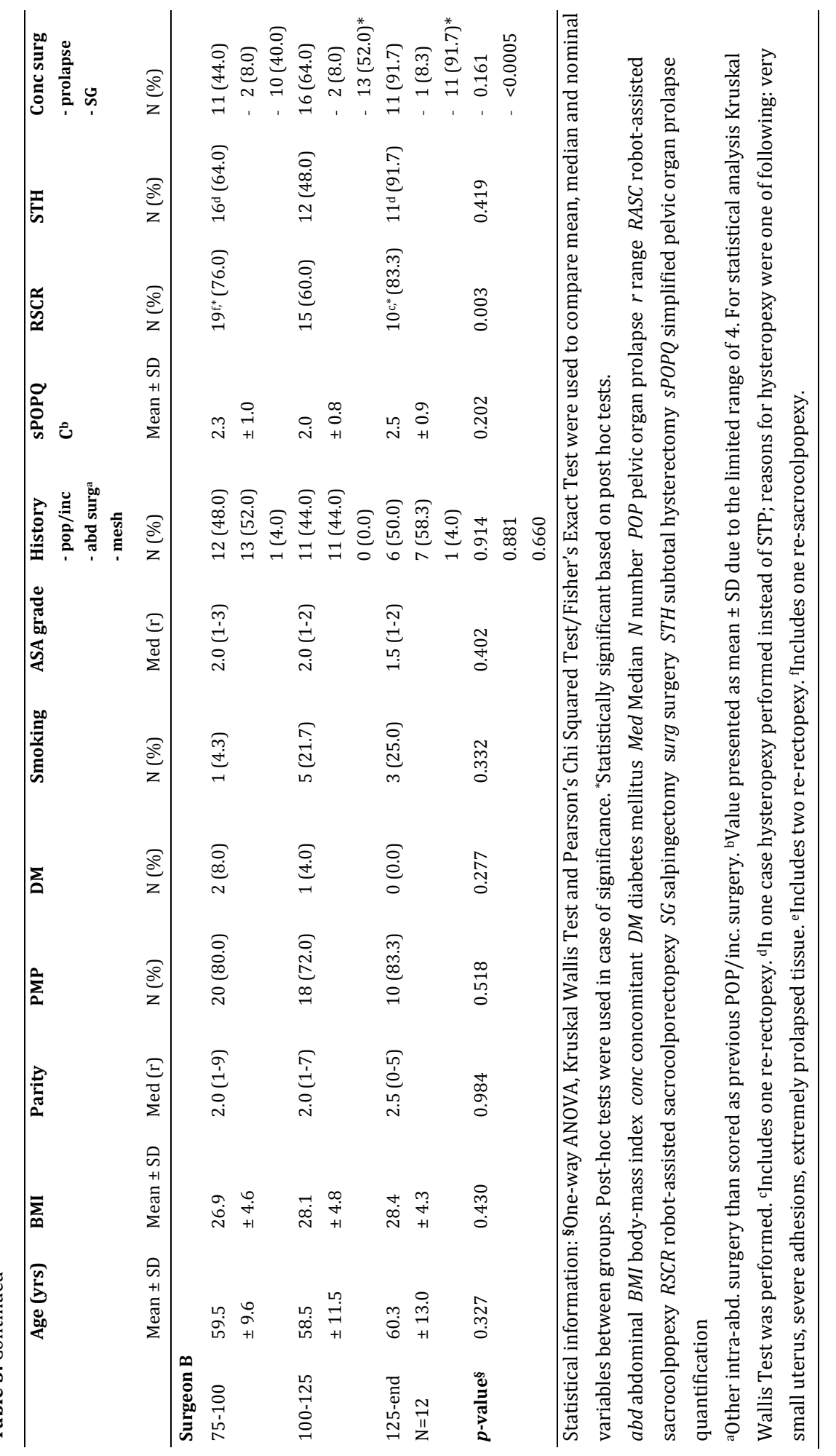


Mean surgery time was $173 \pm 39$ minutes for RASC (surgeon A, $176 \pm 35$ minutes; surgeon $B, 170 \pm 39$ minutes) and $187 \pm 25.8$ for RSCR (surgeon A, $192 \pm 40$ minutes; surgeon $B, 179 \pm 36$ minutes). Moving averages for surgery time are shown in Figure 2 (RASC) and Figure 3 (RSCR). Robot setup time in RASC stabilized after 29 cases, console time and total surgery time plateaued after 26 cases. Console time and total surgery time corresponded well with each other. In RSCR no robot setup time was presented because of missing data. Regarding console and total surgery time, no clear pattern was detectable. CUSUM analysis for total surgery time is shown in Figure 4 (RASC) and Figure 5 (RSCR). In RASC, after 24 cases for surgeon A and after 20-23 cases for surgeon $\mathrm{B}$, surgery time started to diminish. Combining results of MOA and CUSUM analysis, a steady state in surgery time was achieved after 24-29 cases for RASC. Again, no clear pattern could be detected for RSCR. 
Figure 2. Moving average method of surgery time in RASC

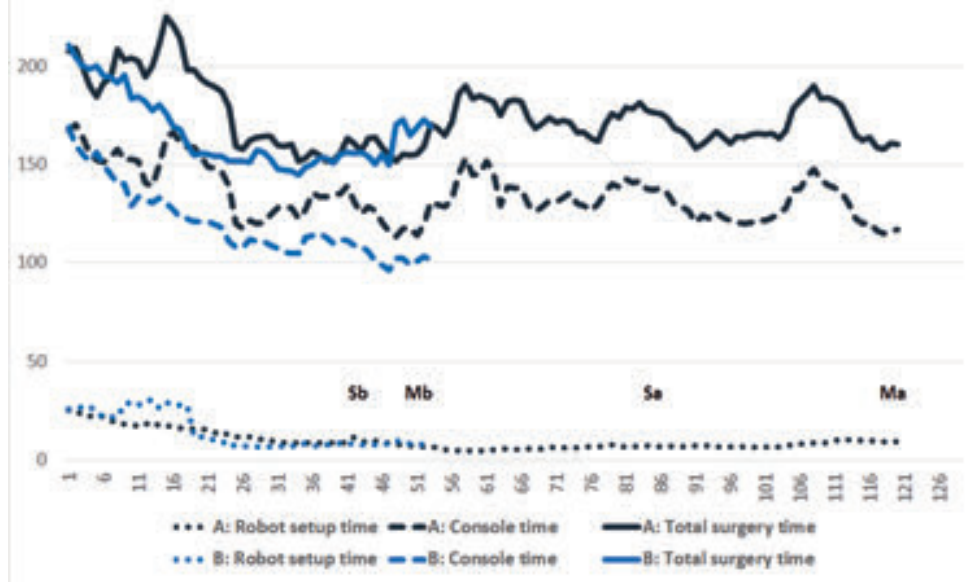

$\mathrm{X}$-axis: number of procedures performed. Y-axis: surgery time (min).

$A$ Surgeon A B Surgeon B Ma start of endobag morcellation surgeon A $M b$ start of endobag morcellation surgeon B $S a$ significantly more salpingectomies performed by surgeon A $S b$ significantly more salpingectomies performed by surgeon B RASC robot-assisted sacrocolpopexy Surgery time was ordered and the mean values were calculated per 10 cases.

Figure 3. Moving average method of surgery time in RSCR

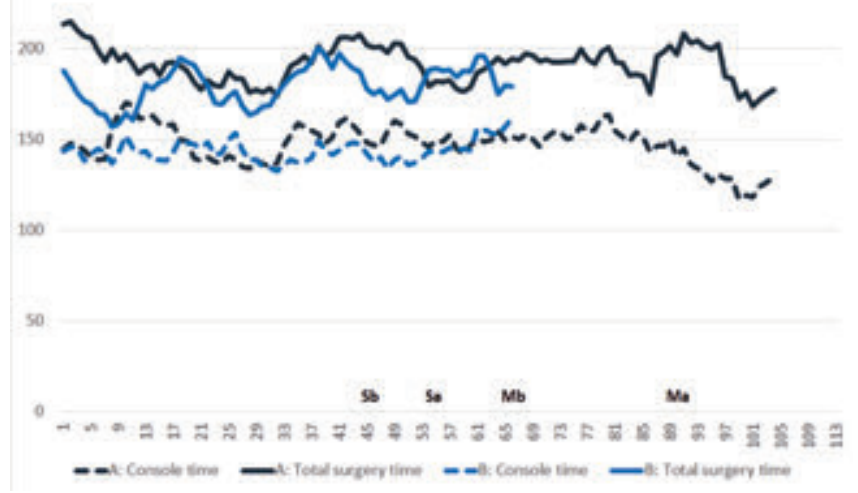

$\mathrm{X}$-axis: number of procedures performed. Y-axis: surgery time (min).

$A$ Surgeon A B Surgeon B A Ma start of endobag morcellation surgeon A $M b$ start of endobag morcellation surgeon B $S a$ significantly more salpingectomies performed by surgeon $S b$ significantly more salpingectomies performed by surgeon B RSCR robot-assisted sacrocolporectopexy Surgery time was ordered and the mean values were calculated per 10 cases. 
Figure 4. CUSUM analysis of total surgery time per surgeon in RASC

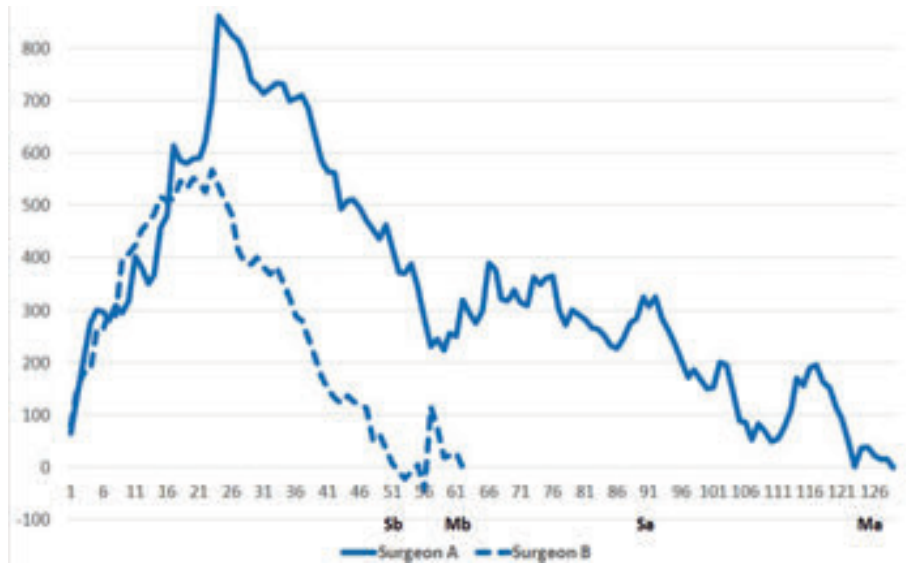

X-axis: number of procedures performed. Y-axis: cumulative surgery time (min). CUSUM cumulative sum analysis $M a$ start of endobag morcellation surgeon A $M b$ start of endobag morcellation surgeon B $S a$ significantly more salpingectomies performed by surgeon A $S b$ significantly more salpingectomies performed by surgeon B RASC robot-assisted sacrocolpopexy In case of perfoming longer or shorter than mean surgery time, the graph respectively rises or falls with the absolute difference in minutes. As rising or falling of the graph is based on mean surgery time, the graph should end on zero minutes again. Surgery time dropped after 20-24 cases.

Figure 5. CUSUM analysis of total surgery time per surgeon in RSCR

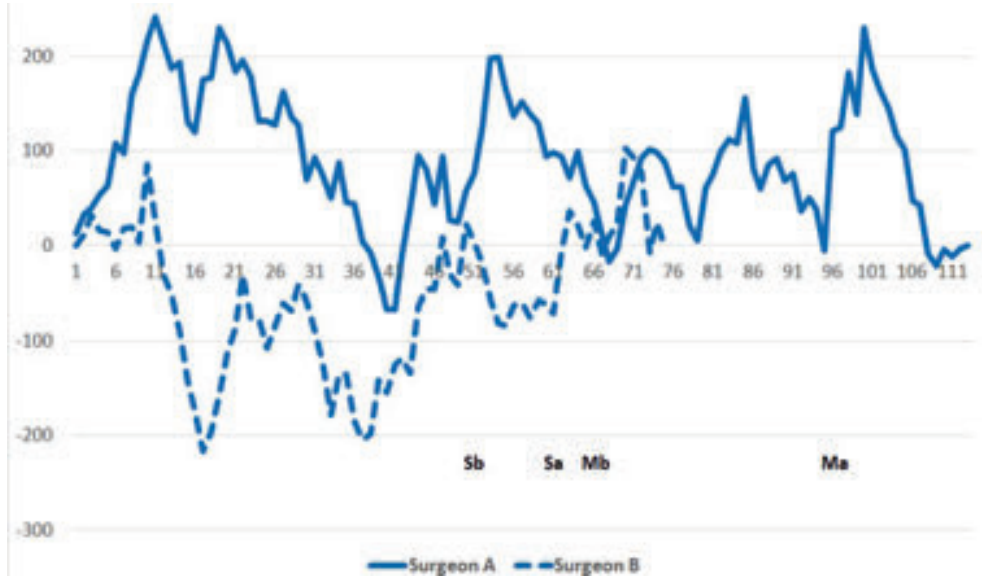

X-axis: number of procedures performed. Y-axis: cumulative surgery time (min). CUSUM cumulative sum analysis $M a$ start of endobag morcellation surgeon A $M b$ start of endobag morcellation surgeon B $S a$ significantly more salpingectomies performed by surgeon A $S b$ significantly more salpingectomies performed by surgeon B RSCR robot-assisted sacrocolporectopexy

In case of perfoming longer or shorter than mean surgery time, the graph respectively rises or falls with the absolute difference in minutes. As rising or falling of the graph is based on mean surgery time, the graph should end on zero minutes again. No clear cutoff point can be estimated. 


\section{Discussion}

\section{Principal findings}

Stabilization of the learning curve regarding intraoperative complications was obtained after 23-41 cases for both surgeons. Proficiency was obtained later, after 78 cases. Intraoperative complications overall were infrequent $(1.9 \%)$ and neither surgeon performed in the unacceptable zone, which suggests that implementation of RASC/RSCR is safe for experienced laparoscopic surgeons. A second trend in complications was seen after 195 cases for surgeon A. Three new complications occurred, although performance remained acceptable. No difference in patient referral pattern could be detected in this period based on baseline demographics. However, there was a difference in surgical technique at the end of the study. The addition of salpingectomy, performing more multicompartment surgeries and the start of endobag morcellation could have influenced performance. This should raise awareness that procedures and patient population can change over time. Monitoring results with the aid of CUSUM analysis could possibly signal this and alert surgeons to potential issues.

Stabilization of total surgery time for RASC occurred between 24-29 cases. Regarding RSCR for multicompartment prolapse, there was no clear increase or decrease of surgery time detectable, which suggests that surgeon's experience is not the key factor for diminishing surgery time in this procedure. Other factors, such as team effort, have been shown to diminish surgery times ${ }^{18}$ and should be examined possibly to improve efficiency in RSCR. A dedicated robotic team during RASC has been described to decrease operative time with $18 \%$. In RSCR, focus of the team should also be on fluently alternating between surgeons.

\section{Results of the study in the context of other observations}

Studies that have reported the learning curve of RASC are scarce and their methods differ. ${ }^{19-24}$ Reduction of mean surgery time of $25 \%$ after 10 cases $^{19}$ and significant shortening of mean total surgery time after 15 cases (mean surgery time, 187 minutes; $\mathrm{n}=40)^{20}$ were reported. This quick drop in reduction of total surgery time is comparable with our findings. Linder et al. ${ }^{21}$ reported that surgery times plateaued after 60 cases. Proficiency was achieved after 55 cases for intraoperative complications and 84 cases for intraoperative or postoperative complications. Risk-adjusted CUSUM analysis was 
used, in which complications that occurred in patients with a higher risk profile (based on American Society of Anesthesiologists, body mass index, number of vaginal deliveries) were scored less heavily. Comparing these numbers for proficiency based on intraoperative complications with our results, our number is slightly higher. Possibly this could be explained by the difference in the applied method of the use of a risk profile. Myers et al. ${ }^{22}$ performed a CUSUM-analysis based on the results of 2 surgeons who performed RASC in a smaller group of patients (surgeon 1, 107; surgeon 2, 62). Both surgeons were performing constantly in the acceptable performance zone, thereby maintaining proficiency the whole time. Their study set the complication target value at $10 \%$, based on previous literature, which is less strict than our target level of $4.4 \%$. Even with our much stricter target level, both surgeons never performed outside of the acceptable performance zone. One systematic review on RASC reports that surgery times drop quickly in the first 10-20 cases based on 2 studies. ${ }^{24}$ Complications were scored, but no specific analysis regarding complications and learning curve was made. CUSUM analysis, especially when intraoperative complications are analyzed, can aid surgeons in receiving feedback on their performance and safety. Even small shifts in surgical performance can be detected, which makes this technique very interesting for the rapidly developing industry. Because a limited amount of studies described the learning curve with aid of CUSUM analysis, our results can be an added value to the literature.

Of studies that describe the learning curve of conventional laparoscopy, ${ }^{25-28}$ Claerhout et al. ${ }^{25}$ used CUSUM analysis. The study included 206 patients who chose conventional laparoscopic sacrocolpopexy. Their primary outcome was failure (laparotomy, complications or anatomical failure $<3$ months). After 60 cases the learning curve was obtained (failure < 10\%). However, failure rate temporarily exceeded the $10 \%$ threshold later in the study, which shows that remaining at a $90 \%$ success rate is a demanding job.

Looking at the scientific literature and our results, reducing surgery time is very different from accomplishing proficiency. To perform significantly better than the acceptable rate $(4.4 \%)$ takes much longer than to lower surgery time. The procedure must be performed many times in order to lower the risk of complications and incidence of injuries. ${ }^{29}$ We found surgery time to decrease first, and then the complication rate was lowered. The difference between these types of learning curves should be acknowledged and taken into account when RASC or RSCR is started. 


\section{Strengths and limitations}

Combining the results of 2 surgeons is a strength of this study. An increase in the CUSUM graph regarding intraoperative complications was seen for surgeon A after 23 cases and for surgeon B after 41 cases. Surgeon B performed fewer surgeries in the same time period. After 2 years surgeon A had performed 87 procedures and surgeon B had performed 49 surgeries. This could have influenced the learning curve because high exposure at regular intervals improves learning. The large number of cases included and use of CUSUM technique are other strengths. This study also has its limitations. Two different procedures were included in the CUSUM analysis. This limited the study homogeneity and therefore may have affected the outcome. Another limitation was that concomitant procedures were included in total surgery time. This was corrected partially by the assessment of sheer console time. However, concomitant subtotal hysterectomy remained within console time. Finally, all complications in CUSUM analysis were of equal weight, whereas there is a difference in the clinical relevance of the different complications.

\section{Clinical implications}

With the advent and integration of new techniques, it becomes more important to have a clear understanding of the learning curves of complex surgical interventions. Knowing surgical pitfalls may prepare surgeons better. More important than describing an absolute number of procedures to obtain proficiency, CUSUM analysis can also be used as a tool to individualize training programs. It can alert surgeons when additional training is necessary. Consensus on surgical training is essential and requirements to begin performing robotic surgery without supervision should be clear. ${ }^{30}$ Results from this study indicate that it might be best to perform the first 23-41 cases with an experienced robotic surgeon. Second, an extensive caseload is necessary to obtain proficiency. It should be assessed if this is possible in every hospital. More research in this field therefore is recommended to set up standardized guidelines eventually.

\section{Conclusion}

Proficiency based on CUSUM analysis in robot-assisted sacrocolpo(recto)pexy was obtained after 78 cases. Stabilization of total surgery time for RASC occurred between 24-29 cases. CUSUM analysis could prove to be a useful tool in training programs that are set up for robot-assisted medical devices to determine robotic proficiency. 


\section{Appendix}

$\mathrm{P}_{0}=$ acceptable failure rate $=0.044$

$\mathrm{P}_{1}=$ unacceptable failure rate $=0.088$

Type 1 error $(\alpha)=$ 'out of control' false $=0.10$

Type 2 error $(\beta)=$ 'in control' false $=0.10$

$\mathrm{a}=\ln [(1-\alpha) / \beta]=\ln (0.9 / 0.1)=\ln 9=2.197$

$\mathrm{b}=\ln [(1-\alpha) / \beta]=\ln (0.9 / 0.1)=\ln 9=2.197$

$\mathrm{P}=\ln \left(\mathrm{P}_{1} / \mathrm{P}_{0}\right)=\ln (0.088 / 0.044)=\ln 2=0.690$

$\mathrm{Q}=\ln \left[\left(1-\mathrm{P}_{0}\right) /\left(1-\mathrm{P}_{1}\right)\right]=\ln [0.956 / 0.912]=\ln 1.048=0.047$

With $\ln =$ natural logarithm $\log _{\mathrm{e}}$.

$\mathrm{s}=\mathrm{Q} /(\mathrm{P}+\mathrm{Q})=0.047 /(0.690+0.047)=0.047 / 0.737=0.064$

Meaning with success, slope goes 0.064 upwards and with failure, slope goes 0.936 (1-s) downwards.

$\mathrm{H}-1$ represent the unacceptable boundary lines: $\mathrm{H}_{-1}=\mathrm{b} /(\mathrm{P}+\mathrm{Q})$

$\mathrm{H}_{1}$ represents the acceptable boundary lines: $\mathrm{H}_{1}=\mathrm{a} /(\mathrm{P}+\mathrm{Q})$

$\mathrm{H}-1=\mathrm{H}_{1}=2.197 /(0.690+0.047)=2.197 / 0.737=2.934$

Notice that since $\alpha=\beta$, this results in $\mathrm{H}_{-1}=\mathrm{H}_{1}$ (space between unacceptable and acceptable boundary lines are equal). To determine the extreme boundary lines $\left(\mathrm{H}_{2}, \mathrm{H}_{3}, \mathrm{H}_{4}\right.$,etc and $\left.\mathrm{H}_{-2}, \mathrm{H}_{-3}, \mathrm{H}_{-4}, \mathrm{etc}\right)$, the same spacing is used. 


\section{References}

1. Carroll AW, Lamb E, Hill AJ, Gill EJ, Matthews CA. Surgical management of apical pelvic support defects : the impact of robotic technology. Int Urogynecol J. 2012;23:1183-1186.

2. Hoekstra AV, Morgan JM, Lurain JR, Buttin BM, Singh DK, Schink JC, et al. Robotic surgery in gynecologic oncology: Impact on fellowship training. Gynecol Oncol. 2009;114:168-172.

3. Steiner SH, Cook RJ. Monitoring surgical performance using risk-adjusted cumulative sum charts. Biostatistics. 2000;1:441-452.

4. Bolsin S, Colson M. The use of the CUSUM Technique in the assessment of trainee competence in new procedures. Int J Qual Health Care. 2000;12:433-438.

5. van Zanten F, Brem C, Lenters E, Broeders IAMJ, Schraffordt Koops SE. Sexual function after robotassisted prolapse surgery: a prospective study. Int Urogynecol J. 2018;29:905-912.

6. van Iersel JJ, de Witte CJ, Verheijen PM, et al. Robot-assisted sacrocolporectopexy for multicompartment prolapse of the pelvic floor: a prospective cohort study evaluating functional and sexual outcome. Dis Colon Rectum. 2016;59:968-974.

7. Morena Sierra J, Ortiz Oshiro E, Fernandeze Pérez C, et al. Long-term outcomes after robotic sacrocolpopexy for vaginal prolapse: prospective analysis. Urol Int. 2011;86:414-418.

8. Paraiso MFR, Jelovsek JE, Frick A, Chen CCG, Barber MD. Laparoscopic compared with robotic sacrocolpopexy for vaginal prolapse: a randomized controlled trial. Obstet Gynecol. 2011;118:1005-1013.

9. Matthews CA, Carroll A, Hill A, Ramakrishnan V, Gill Ej. Prospective evaluation of surgical outcomes of robot-assisted sacrocolpopexy and sacrocervicopexy for the management of apical pelvic support defects. South Med J. 2012;105:274-8.

10. Seror J, Yates DR, Seringe E, Vaessen C, Bitker M-0, Chartier-Kastler E, et al. Prospective comparison of short-term functional outcomes obtained after pure laparoscopic and robotassisted laparoscopic sacrocolpopexy. World J Urol. 2012;30:393-398.

11. Culligan PJ, Gurshumov E, Lewis C, Priestly JL, Komar J, Shah N, et al. Subjective and objective results 1 year after robotic sacrocolpopexy using a lightweight Y-mesh. Int Urogynecol J. 2014;25:731-735.

12. Woelk JL, Casiano ER, Weaver AL, Gostout BS, Trabuco EC, Gebhart JB. The learning curve of robotic hysterectomy. Obstet Gynecol. 2013;121:87-95.

13. Satava RM. Identification and reduction of surgical error using simulation. Minim Invasive Ther Technol. 2005;14:257-261.

14. Swift S, Morris S, McKinnie V, Freeman R, Petri E, Scotti RJ, et al. Validation of a simplified technique for using the POPQ pelvic organ prolapse classification system. Int Urogynecol J. 2006;17:615-620.

15. Nezhat FR, Apostol R, Nezhat C, Pejovic T. New insights in the pathophysiology of ovarian cancer and implications for screening and prevention. Am J Obstet Gynecol. 2015;213:262-267.

16. Rimbach S, Schempershofe M. In-Bag Morcellation as a Routine for Laparoscopic Hysterectomy. BioMed Res Int. 2017;Article ID 6701916:1-6.

17. Tan-Kim J, Hartzell KA, Reinsch CS, et al. Uterine sarcomas and parasitic myomas after laparoscopic hysterectomy with power morcellation. Am J Obstet Gynecol. 2015;212:594.e1-e10. 
18. Carter-Brooks CM, Du AL, Bonidie MJ, Shepherd JP. The impact of a dedicated robotic team on robotic-assisted sacrocolpopexy outcomes. Female Pelvic Med Reconstr Surg. 2018;24:13-16.

19. Akl MN, Long JB, Giles DL, Cornella JL, Pettit PD, Chen AH, et al. Robotic-assisted sacrocolpopexy: Technique and learning curve. Surg Endosc. 2009;23:2390-2394.

20. Awad N, Mustafa S, Amit A, Deutsch M, Eldor-Itskovitz J, Lowenstein L. Implementation of a new procedure: Laparoscopic versus robotic sacrocolpopexy. Arch Gynecol Obstet. 2013;287:11811186.

21. Linder BJ, Anand M, Weaver AL, Woelk JL, Klingele CJ, Trabuco EC, et al. Assessing the learning curve of robotic sacrocolpopexy. Int Urogynaecol J. 2016;27:239-246.

22. Myers EM, Geller EJ, Connolly A, Bowling JM, Matthews CA. Robotic sacrocolpopexy performance and cumulative summation analysis. Female Pelvic Med Reconstr Surg. 2014;20:83-86.

23. Sharma S, Calixte R, Finamore PS. Establishing the learning curve of robotic sacral colpopexy in a start-up robotics program. J Minim Invasive Gynecol. 2016;23:89-93.

24. Serati M, Bogani G, Sorice P, Braga A, Torella M, Salvatore S, et al. Robot-assisted sacrocolpopexy for pelvic organ prolapse: a systematic review and meta-analysis of comparative studies. Eur Urol. 2014;66:303-318.

25. Claerhout F, Roovers JP, Lewi P, Verguts J, De Ridder D, Deprest J. Implementation of laparoscopic sacrocolpopexy-a single centre's experience. Int Urogynecol J. 2009;20:1119-1125.

26. Vandendriessche D, Giraudet G, Lucot JP, Behal H, Cosson M. Impact of laparoscopic sacrocolpopexy learning curve on operative time, perioperative complications and short term results. Eur J Obstet Gynecol Reprod Biol. 2015;191:84-89.

27. Akladios CY, Dautun D, Saussine C, Baldauf JJ, Mathelin C, Wattiez A. Laparoscopic sacrocolpopexy for female genital organ prolapse: establishment of a learning curve. Eur J Obstet Gynecol Reprod Biol 2010;149:218-221.

28. Claerhout F, Verguts J, Werbrouck E, Veldman J, Lewi P, Deprest J. Analysis of the learning process for laparoscopic sacrocolpopexy: identification of challenging steps. Int Urogynecol J. 2014;25:1185-1191.

29. Moawad G, Tyan P, Corpodean F, Robinson J. Ethical considerations arising from surgeon caseload volume in benign gynecologic surgery. J Minim Invasive Gynecol. 2018;25:749-751.

30. Sinha R, Sanjay M, Rupa B, Kumari S. Robotic surgery in gynecology. J Min Access Surg. 2015;11:50-59. 



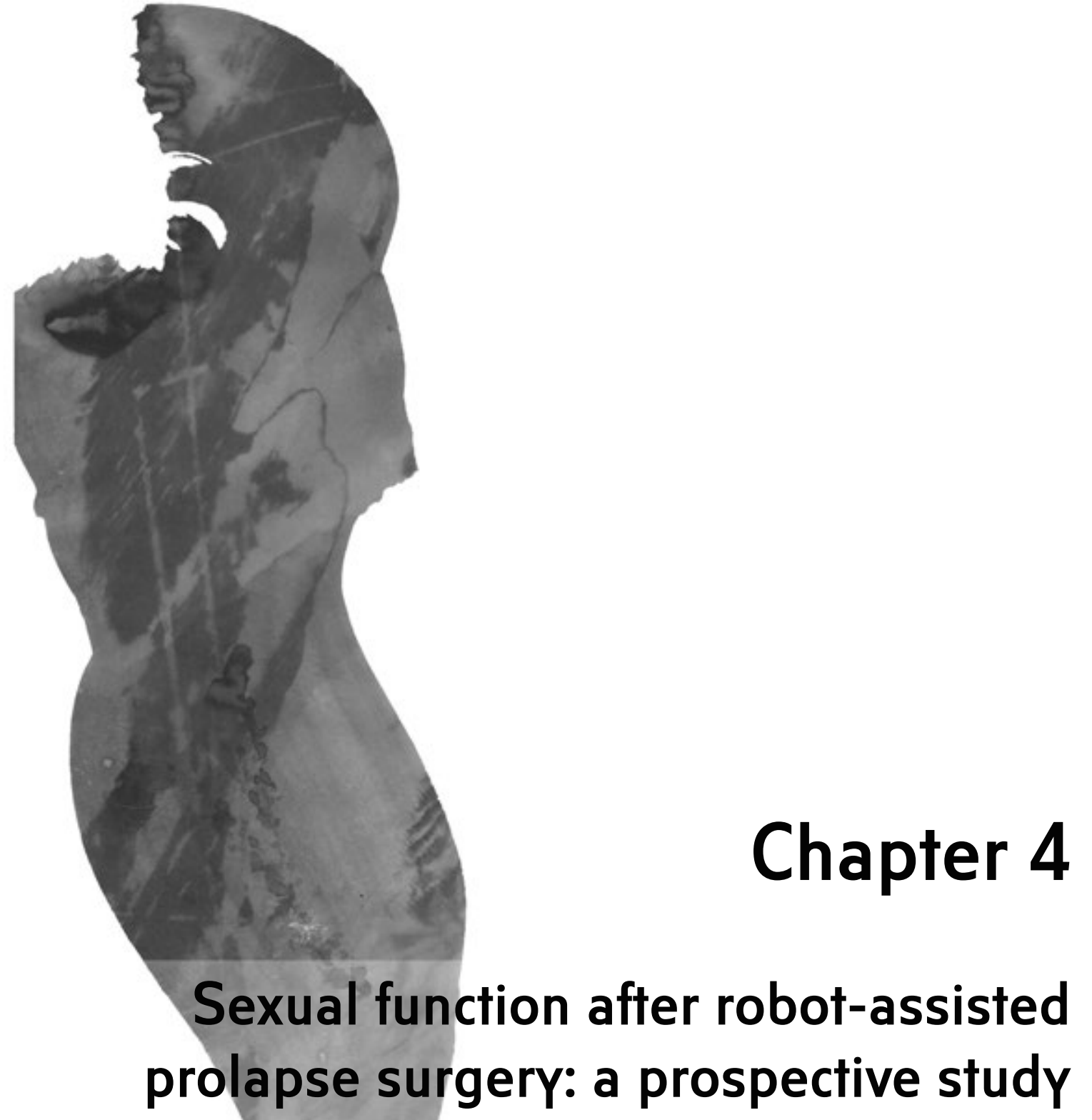

Femke van Zanten, Cherèl Brem, Egbert Lenters Ivo A.M.J. Broeders, Steven E. Schraffordt Koops 


\section{Abstract}

Introduction and hypothesis Female pelvic organ prolapse (POP) can severely influence sexual function. Robot-assisted surgery is increasingly used to treat POP, but studies describing its effect on sexual function are limited. The objective of this study was to evaluate sexual function after robot-assisted POP surgery.

Methods This prospective cohort study included all patients who underwent a robotassisted sacrocolpopexy (RASC) or supracervical hysterectomy with sacrocervicopexy (RSHS). Exclusion criteria were unknown preoperative sexual activity status or concomitant surgery. In sexually active women, sexual function was measured with the translated validated version of the Pelvic Organ Prolapse/Urinary Incontinence Sexual Questionnaire (PISQ-12). Changes in sexual activity were scored. Prolapse stages were described using the simplified Pelvic Organ Prolapse Quantification system.

Results A total of 107 women were included (median follow-up 15.3 months). No difference was found in the total number of sexually active women before and after surgery [63 (58.9\%) vs. 61 (63.5\%), $p=0.999$ ]. Significantly fewer women avoided sexual intercourse postoperatively compared with preoperatively. Preoperatively, sexual intercourse was avoided due to vaginal bulging ( $2 \%$ vs. $24 \%$, respectively, $p=0.021$ ). Total mean PISQ-12 scores improved significantly 1 year after prolapse correction (33.5 versus $37.1 ; p=0.004$ ), mainly due to improved scores on the physical and behavioralemotive domain. No significant difference in pre- and postoperative complains of dyspareunia was found.

Conclusion Robot-assisted middle compartment surgery improved sexual function 1 year after surgery according to enhanced physical and emotional scores. The total number of sexually active women and complains of dyspareunia before and after surgery did not differ. 


\section{Introduction}

An increasing number of women suffer from pelvic organ prolapse (POP). ${ }^{1}$ This condition is known to negatively impact sexual functioning and quality of life (QoL). ${ }^{2,3}$ Sexual dysfunction as a result from POP can be caused by the sensation of vaginal pressure, dyspareunia, and/or embarrassment during sexual activity. Laparoscopic sacrocolpopexy (LSC) is the gold standard for the treatment of apical/vault prolapse. ${ }^{4}$ The open procedure was first reported by Lane in 1962 for women suffering from vaginal vault prolapse following total hysterectomy. ${ }^{5}$ In search of a technique in which vaginal function post hysterectomy was not lost, Lane proposed to anchor the prolapsed vaginal vault to the sacral promontory with the use of synthetic material. This led eventually to the abdominal sacrocolpopexy as we know it today. Throughout the years several studies have shown that sacrocervicopexy following a subtotal hysterectomy is an effective treatment for uterine prolapse.6,7 Investigating the influence of these middle-compartment operative prolapse techniques on sexual function, and therefore QoL, is an important issue.

Robot-assisted prolapse surgery is increasingly used due to the technical advantages of the robot. Three-dimensional vision, physiologic tremor filtering, increased freedom of instrumental movement, and optimal ergonomics characterize the robot-assisted approach. These factors may help the surgeon perform a deep and precise dissection in the pelvis and anchor the mesh over the prolapsed walls as much as possible in an effort to minimize recurrence and mesh related complications. Despite the increasing use of robotics for apical prolapse correction, studies describing sexual function after robotassisted sacrocolpopexy (RASC) are scarce. ${ }^{8-11}$ Considering this lack of studies on sexual function after robot-assisted abdominal prolapse surgery, we performed a prospective, large cohort study using the translated and validated version of the Pelvic Organ Prolapse/Urinary Incontinence Sexual Questionnaire (PISQ-12) as a primary outcome measurement. ${ }^{3}$

\section{Materials and Methods}

This study is part of the Prospective Assessment of Robotic Sacrocolpopexy: a European Multicentric Cohort (PARSEC) database and a planned ancillary analysis (https://clinicaltrials.gov; identifier NCT01598467). All patients suffering from vaginal middle compartment prolapse treated with RASC or robot-assisted supracervical 
hysterectomy with a sacrocervicopexy (RSHS) between 2012 and 2015 were prospectively included. Prolapse stages were described using the simplified Pelvic Organ Prolapse Quantification (SPOPQ).12,13 The SPOPQ is a validated short form of the standard POP-Q system. It describes only four vaginal landmarks (anterior and posterior vaginal walls, cervix/vaginal cuff, posterior fornix) instead of nine. Only women with SPOPQ stage $\geq 2$ (stage 2: given point of the SPOPQ descends between $1 \mathrm{~cm}$ above to 1 $\mathrm{cm}$ below the hymenal remnants) were included. Patients with no documented preoperative sexual activity status were excluded. To eliminate the influence of concomitant surgeries on sexual function, patients undergoing concomitant surgery were excluded (anterior and/or posterior colporrphaphy, rectopexy, anti-incontinence surgery, and oophorectomy). Patients were considered lost to follow-up if their sexual activity status postoperatively was unknown.

Sexual function was measured by scoring sexual activity status (i.e. sexually active versus inactive) and by using the translated and validated Dutch version of the Pelvic Organ Prolapse/Urinary Incontinence Sexual Questionnaire (PISQ-12). ${ }^{3}$ The PISQ-12 was given to all patients before and 1 year after surgery. All patients were seen preoperatively in the outpatient clinic and 6 weeks and 1 year after surgery. Patients filled in the PISQ-12 independently and the questionnaire was handed in during the 1year clinical evaluation. In case patients refused or were not able to visit the clinic, the questionnaire was sent by mail. The PISQ-12 evaluates sexual function in women with urinary incontinence and/or pelvic organ prolapse (POP). ${ }^{14}$ It is the validated short form of the PISQ-31 and therefore more manageable for patients to answer. The PISQ-12 contains 12 questions concerning sexual functioning in three domains: behavioralemotive (items 1-4), physical (items 5-9) and partner-related (items 10-12). ${ }^{3}$ Five response options were provided (always, mostly, sometimes, seldom, never). The scores of the first four questions ranged from 0 'never' to 4 'always' and the last eight questions ranged from 0 'always' to 4 'never'. The sum of all answers was computed to form a total score (range 0-48), with higher scores indicating better sexual function. Questionnaires with up to two missing answers were accepted, and missing items were replaced with the mean of all answered items. The PISQ-12 is not validated for patients who are sexually inactive. In such cases, this was listed in their medical record to detect changes in sexual activity before and after surgery. To allow for comparison of the PISQ-12 scores, only women who filled in the questionnaire both before and after surgery were analyzed. 
Each PISQ-12 item was divided to describe percentages, in which answers of 'always or mostly' were considered as one group, 'sometimes' as a second group and 'seldom or never' as the third group. De novo dyspareunia was defined when dyspareunia preoperatively was scored 'seldom or never' and postoperatively 'always or mostly'.

Deterioration and improvement ratios according to the PISQ-12 in individual sexually active patients were calculated using the following formula: [(total PISQ-12 score followup) - (total PISQ-12 score preoperative)] / (total PISQ-12 score preoperative) x 100. Ratios were considered stable if they changed by $<10 \%$, as improved if they increased by $10-50 \%$, very improved if they increased by $>50 \%$, worsened if they decreased by 10 $50 \%$, and very worsened if they decreased by $>50 \%$. Ultimately, separate scores for the two surgical techniques (RASC and RSHS) and for two different age groups $(\leq 60$ and $>60$ ) were given, as more recurrent prolapses may be seen in the age group of $\leq 60$ years after prolapse surgery. ${ }^{15}$

All surgical procedures were performed by experienced gynecologists using the da Vinci ${ }^{\circledR}$ Robot (Intuitive Surgical, Inc., Sunnyvale, CA, USA). The technique used to perform pelvic floor correction was similar to that described by Clifton et al. ${ }^{16}$ In patients with the uterus present, a subtotal hysterectomy was performed. For prolapse correction, macroporous polypropylene mesh (type 1, Prolene, Ethicon Inc., Johnson \& Johnson, Hamburg, Germany) was used. The mesh was sutured to the anterior and posterior vaginal wall with non-absorbable sutures (Ethibond, Ethicon Inc., Johnson \& Johnson, Hamburg, Germany) and intracorporeally configured into a $Y$ shape. The mesh was anchored proximally to the sacral promontory using titanium tacks (Autosuture Protack 5mm, Covidien, USA).

This study was carried out in accordance with the ethical standards of The National Central Committee on Research Involving Human Subjects (CCMO). Statistical analysis was performed using IBM SPSS statistics version 22.0. Mean values were compared using the two-sided independent or paired samples t-test and median values using a Mann Whitney $U$ Test or Wilcoxon signed-rank test, as appropriate. Nominal or ordinal data were analyzed with a chi- squared, Fisher's exact, or McNemar tests. An alpha value of 0.05 was considered significant. 


\section{Results}

One hundred and nineteen patients underwent eligible surgery. In 12 patients, sexual activity was unknown at baseline, resulting in a final sample size of 107 patients (Figure 1). Sixty-three patients (58.9\%) were sexually active and 44 patients (41.1\%) sexually inactive. Eleven of the 107 patients (10.3\%) were lost to follow-up (patient preference because of no complains $n=5$, incorrect contact details $n=1$, no reason/other $n=5$ ). Of the remaining 96 participants, $92(95.8 \%)$ were seen postoperatively in the outpatient clinic, and 4 (4.2\%) sent in the completed questionnaire by post. The median follow-up duration was 15.3 months (range 8.9-44.4). The baseline characteristics of all patients are listed in Table 1. RASC was performed on 45 patients (42\%; including two conversions to an open abdominal sacrocolpopexy) and RSHS on 62 (58\%) patients. Almost half of all patients (44.9\%) had a history of prolapse or anti-incontinence surgery, and $82.2 \%$ were postmenopausal. The use of preoperative vaginal estrogens was low (3.7\%). Sexually active and inactive patients were compared on various demographic characteristics. These two groups differed in age, postmenopausal status and mean SPOPQ C (middle compartment). A more severe prolapse was seen in sexually inactive women (Table 1).

Figure 1. Flow chart of included patients

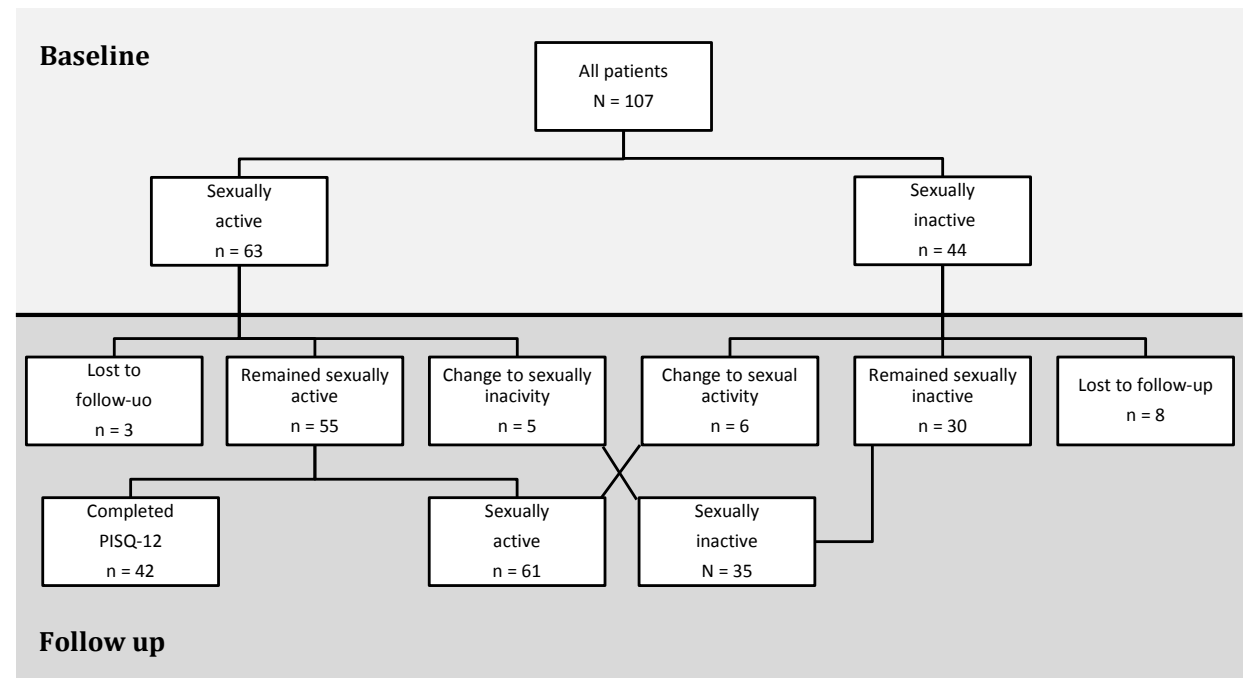

$N$ number PISQ-12 Pelvic Organ Prolapse/Urinary Incontinence Sexual Questionnaire 
Table 1. Baseline patient characteristics

\begin{tabular}{|c|c|c|c|c|}
\hline Characteristics & $\begin{array}{l}\text { All } \\
\text { patients } \\
(\mathrm{N}=107)\end{array}$ & $\begin{array}{l}\text { Sexually } \\
\text { active } \\
(n=63)\end{array}$ & $\begin{array}{l}\text { Sexually } \\
\text { inactive } \\
(n=44)\end{array}$ & $\begin{array}{l}p \\
\text { value }^{a}\end{array}$ \\
\hline Age $(y)$, mean \pm SD (range) & $61.5 \pm 10.6$ & $58.8 \pm 10.1$ & $65.4 \pm 10.2$ & $0.001^{\mathrm{b}}$ \\
\hline $\begin{array}{l}\mathrm{BMI}\left(\mathrm{kg} / \mathrm{m}^{2}\right) \text {, } \\
\text { median (range) }\end{array}$ & $\begin{array}{l}25.5 \\
(19.6-37.8)\end{array}$ & $\begin{array}{l}25.1 \\
(19.8-35.5)\end{array}$ & $\begin{array}{l}26.8 \\
(19.6-37.8)\end{array}$ & 0.093 \\
\hline Parity, median (range) & $3.0(0-7)$ & $2.5(1-7)$ & $3.0(0-6)$ & 0.208 \\
\hline Postmenopausal, N (\%) & $88(82.2)$ & $47(74.6)$ & $41(93.2)$ & $0.027^{b}$ \\
\hline \multicolumn{5}{|l|}{ sPOPQ, mean (range) } \\
\hline $\mathrm{Ba}$ & $2.9(1-4)$ & $2.8(1-4)$ & $3.0(1-4)$ & 0.382 \\
\hline Bp & $2.1(1-4)$ & $2.0(1-4)$ & $2.3(1-4)$ & 0.236 \\
\hline $\mathrm{C}$ & $2.9(1-4)$ & $2.7(1-4)$ & $3.1(1-4)$ & $0.046^{\mathrm{b}}$ \\
\hline D & $2.2(1-4)$ & $1.9(1-4)$ & $2.5(1-4)$ & 0.103 \\
\hline Previous intra-abdominal surgery, ${ }^{c} \mathrm{~N}(\%)$ & $72(67.3)$ & $40(63.5)$ & $32(72.7)$ & 0.316 \\
\hline Previous prolapse/incontinence surgery, $\mathrm{N}(\%)$ & $48(44.9)$ & $29(46.0)$ & $19(43.2)$ & 0.771 \\
\hline Previous hysterectomy, N (\%) & $45(42.1)$ & $23(36.5)$ & $22(50.0)$ & 0.164 \\
\hline Dyspareunia, $\mathrm{N}(\%)$ & $\mathrm{N} / \mathrm{A}$ & $25(39.7)$ & $\mathrm{N} / \mathrm{A}$ & $\mathrm{N} / \mathrm{A}$ \\
\hline Symptoms of bulge, $\mathrm{N}(\%)$ & $103(96.3)$ & $61(96.8)$ & $42(95.5)$ & 0.999 \\
\hline Preoperative use of vaginal estrogens, $N(\%)$ & $4(3.7)$ & $1(1.6)$ & $3(6.8)$ & 0.303 \\
\hline
\end{tabular}

Sexual function measured with PISQ-12

Forty-two of the 55 patients (76.4\%) who were sexually active both before and after surgery filled in a pre- and postoperative PISQ-12. The other patients were either sexually inactive pre-operatively $(n=44)$ or changed from being sexually active to sexually inactive postoperatively $(n=5)$, and could therefore not complete a PISQ-12. Furthermore, 3 patients were lost to follow-up (Figure 1). Total PISQ-12 scores improved significantly from 33.5 to 37.1 ( $p=0.004)$. Of the 12 separate items, 2 showed a significant improvement in orgasm frequency and avoidance of sexual activity because of symptoms of vaginal bulging (Table 2). Both the physical and the behavioral-emotive domain showed overall significant improvement in mean scores $[ \pm$ standard deviation (SD)] scores (respectively, $2.9 \pm 0.9$ vs $3.5 \pm 0.6 ; p=0.003$ and $2.5 \pm 0.7$ vs $2.8 \pm 0.7$; $p=0.029)$. No difference was seen in the partner related domain $(2.9 \pm 0.6$ vs $2.9 \pm 0.6$; $p=0.804)$. 


\begin{tabular}{|c|c|c|c|}
\hline & $\begin{array}{l}\text { Preoperative } \\
\text { baseline } \\
(n=42)\end{array}$ & $\begin{array}{l}1 \text { year after } \\
\text { surgery } \\
(n=42)\end{array}$ & $p$ value \\
\hline \#1: Sexual desire & $2.3(0.7)$ & $2.4(0.8)$ & 0.675 \\
\hline \#2: Orgasm frequency & $2.3(1.1)$ & $2.7(1.0)$ & $0.044^{b}$ \\
\hline \#3: Arousal & $2.8(1.0)$ & $3.0(0.9)$ & 0.146 \\
\hline \#4: Satisfaction variety sexual activities & $2.7(1.0)$ & $3.0(0.9)$ & 0.110 \\
\hline \#5: Dyspareunia & $2.5(1.5)$ & $2.7(1.4)$ & 0.391 \\
\hline $\begin{array}{l}\text { \#6: Urinary incontinence during sexual } \\
\text { activities }\end{array}$ & $3.3(1.1)$ & $3.6(0.9)$ & 0.104 \\
\hline $\begin{array}{l}\text { \#7: Fear of fecal or urinary incontinence } \\
\text { during sexual activities }\end{array}$ & $3.3(1.2)$ & $3.7(0.7)$ & 0.104 \\
\hline $\begin{array}{l}\text { \#8: Avoidance of sexual activity because } \\
\text { of symptoms of vaginal bulging }\end{array}$ & $2.6(1.4)$ & $3.8(0.6)$ & $<0.0005^{b}$ \\
\hline $\begin{array}{l}\text { \#9: Negative emotions during sexual } \\
\text { intercourse }\end{array}$ & $3.1(1.1)$ & $3.6(0.9)$ & 0.063 \\
\hline \#10: Erection problem partner & $3.4(0.9)$ & $3.5(1.0)$ & 0.393 \\
\hline \#11: Premature ejaculation partner & $3.6(0.7)$ & $3.6(0.8)$ & 0.762 \\
\hline \#12: Change in orgasm intensity & $1.6(1.0)$ & $1.7(0.7)$ & 0.618 \\
\hline Total PISQ-12 score & $33.5(5.6)$ & $37.1(5.4)$ & $0.004^{\mathrm{b}}$ \\
\hline \multicolumn{4}{|c|}{ Data presented as mean (SD) \# Question number PISQ-12 Pelvic Organ Prolapse/Urinary Incontinence } \\
\hline $\begin{array}{l}\text { Sexual Questionnaire } S D \text { standard deviatior } \\
\text { significant. }\end{array}$ & ${ }^{a}$ Questions of th & 12 are shorten & tically \\
\hline
\end{tabular}

The individual questions of the PISQ-12 were converted into percentages and these results are depicted in Table 3. Preoperatively, almost a quarter of all sexually active women (24\%) avoided sex always or mostly because of symptoms of vaginal bulging. This number significantly decreased to $2 \% 1$ year after prolapse correction $(p=0.021)$. Similarly, the number of women suffering from dyspareunia decreased after surgery, from 29 to 17\%. Patients who reported to have dyspareunia sometimes increased from 10 to $26 \%$. Item 5, reporting the mean score of dyspareunia, improved, but not significantly (Table 2). De novo dyspareunia occurred in 2 patients (4.7\%). One suffered from vaginal atrophy; neither had a mesh exposure. In total, 7 patients (6.5\%) started using vaginal estrogens 6 weeks after surgery and continued use until follow-up 1 year later. Four patients (3.7\%) were advised to start using vaginal estrogens at their 1-year consultation. In total, one patient suffered from a small vaginal mesh exposure. She had no complaints of dyspareunia either before or after surgery. 
Table 3. PISQ-12 items as percentages

\begin{tabular}{|c|c|c|c|c|c|c|c|}
\hline & \multicolumn{3}{|c|}{$\begin{array}{l}\text { Preoperative baseline } \\
(n=42)\end{array}$} & \multicolumn{3}{|c|}{$\begin{array}{l}1 \text { year after surgery } \\
(n=42)\end{array}$} & \multirow[t]{2}{*}{$p$ value $^{a}$} \\
\hline & $\begin{array}{l}\text { Always } \\
\text { or } \\
\text { Mostly }\end{array}$ & $\begin{array}{l}\text { Some- } \\
\text { times }\end{array}$ & $\begin{array}{l}\text { Seldom } \\
\text { or } \\
\text { Never }\end{array}$ & $\begin{array}{l}\text { Always } \\
\text { or } \\
\text { Mostly }\end{array}$ & $\begin{array}{l}\text { Some- } \\
\text { times }\end{array}$ & $\begin{array}{l}\text { Seldom } \\
\text { or } \\
\text { Never }\end{array}$ & \\
\hline \#1: Sexual desire & 43 & 45 & 12 & 48 & 38 & 12 & 0.999 \\
\hline \#2: Orgasm frequency & 41 & 38 & 21 & 67 & 21 & 12 & 0.063 \\
\hline \#3: Arousal & 62 & 24 & 7 & 79 & 14 & 7 & 0.999 \\
\hline $\begin{array}{l}\text { \#4: Satisfaction variety } \\
\text { sexual activities }\end{array}$ & 69 & 10 & 14 & 81 & 5 & 12 & 0.999 \\
\hline \#5: Dyspareunia & 29 & 10 & 62 & 17 & 26 & 57 & 0.687 \\
\hline $\begin{array}{l}\text { \#6: Urinary incontinence } \\
\text { during sexual activities }\end{array}$ & 10 & 12 & 79 & 5 & 5 & 90 & 0.687 \\
\hline $\begin{array}{l}\text { \#7: Fear of fecal or urinary } \\
\text { incontinence during sexual } \\
\text { activities }\end{array}$ & 10 & 12 & 79 & 2 & 7 & 88 & 0.375 \\
\hline $\begin{array}{l}\text { \#8: Avoidance of sexual } \\
\text { activity because of symptoms } \\
\text { of vaginal bulging }\end{array}$ & 24 & 21 & 50 & 2 & 2 & 91 & $0.021^{b}$ \\
\hline $\begin{array}{l}\text { \#9: Negative emotions } \\
\text { during sexual intercourse }\end{array}$ & 7 & 21 & 71 & 5 & 7 & 88 & 0.999 \\
\hline $\begin{array}{l}\text { \#10: Erection problem } \\
\text { partner }\end{array}$ & 2 & 21 & 76 & 10 & 5 & 86 & 0.999 \\
\hline $\begin{array}{l}\text { \#11: Premature ejaculation } \\
\text { partner }\end{array}$ & 2 & 10 & 88 & 2 & 12 & 83 & 0.999 \\
\hline $\begin{array}{l}\text { \#12: Change in orgasm } \\
\text { intensity }\end{array}$ & 17 & 31 & 50 & 7 & 60 & 31 & 0.999 \\
\hline \multicolumn{8}{|l|}{ PISQ-12 questions are shortened. } \\
\hline \multicolumn{8}{|l|}{ Data presented as $\%$} \\
\hline \multicolumn{8}{|c|}{ \# Question number PISQ-12 Pelvic Organ Prolapse/Urinary Incontinence Sexual Questionnaire } \\
\hline $\begin{array}{l}\text { aComparing preoperative 'alway } \\
\text { and 'seldom or never'. bStatistica }\end{array}$ & s or mostl & $\begin{array}{l}\text { and 'seld } \\
\text { nt. }\end{array}$ & om or nev & er' with p & stoperat & ve 'always & or mostly' \\
\hline
\end{tabular}

After surgery 48 and $7 \%$ of patients' sexual function was improved and very improved respectively, and remained stable in 31\%. In 6 women (14\%) sexual function worsened, in 3 of these patients a recurrent prolapse was diagnosed (Table 4). In subgroup analyses (age and surgery type) no statistically significant differences were seen in deterioration and amelioration of sexual function (Table 4). No difference was seen in total PISQ-12 scores pre- and postoperative when comparing the two age groups and 
surgical technique [preoperative: 33.9 ( $\leq 60)$ vs 32.3 (>60), $p=0.343$; postoperative: 37.5 ( $\leq 60$ ) vs 37.0 (>60), p=0.741; preoperative: 32.4 (RASC) vs 33.7 (RSHS), $p=0.416$; postoperative 37.0 (RASC) vs 37.5 (RSHS), $p=0.726$ ).

Table 4. Deterioration and amelioration of total PISQ-12 scores

\begin{tabular}{llllllll}
\hline & $\begin{array}{l}\text { All patients } \\
(\mathbf{n = 4 2 )}\end{array}$ & $\begin{array}{l}\text { Age } \leq \mathbf{6 0} \\
(\mathbf{n = 2 4 )}\end{array}$ & $\begin{array}{l}\text { Age }>\mathbf{6 0} \\
\mathbf{( n = 1 8 )}\end{array}$ & $\begin{array}{l}\boldsymbol{p} \\
\text { value }^{\mathbf{a}}\end{array}$ & $\begin{array}{l}\text { RASC } \\
(\mathbf{n = 1 8})\end{array}$ & $\begin{array}{l}\text { RSHS } \\
(\mathbf{n = 2 4 )}\end{array}$ & $\begin{array}{l}\boldsymbol{p} \\
\text { value }^{\mathbf{b}}\end{array}$ \\
\hline Very improved & $7.1 \%(3)$ & $4.2 \%(1)$ & $11.1 \%(2)$ & 0.567 & $16.7 \%(3)$ & $0 \%(0)$ & 0.071 \\
Improved & $47.6 \%(20)$ & $54.2 \%(13)$ & $38.9 \%(7)$ & 0.327 & $33.3 \%(6)$ & $58.3 \%(14)$ & 0.108 \\
Stable & $31.0 \%(13)$ & $33.3 \%(8)$ & $27.8 \%(5)$ & 0.700 & $33.3 \%(6)$ & $29.2 \%(7)$ & 0.773 \\
Worsened & $14.3 \%(6)$ & $8.3 \%(2)$ & $22.2 \%(4)$ & 0.375 & $16.7 \%(3)$ & $12.5 \%(3)$ & 0.999 \\
Very worsened & $0.0 \%(0)$ & $0.0 \%(0)$ & $0.0 \%(0)$ & N/A & $0.0 \%(0)$ & $0.0 \%(0)$ & N/A \\
\hline
\end{tabular}

Data presented as \% (N)

N/A not applicable PISQ-12 Pelvic Organ Prolapse/Urinary Incontinence Sexual Questionnaire RASC robot-assisted sacrocolpopexy RSHS robot-assisted supracervical hysterectomy with sacrocervicopexy ${ }^{a}$ Comparing age $\leq 60$ with age $>60$. ${ }^{b}$ Comparing RASC with RSHS.

\section{Changes in sexual activity status}

No significant differences were found in the number of sexually active women before and after surgery [63 (58.9\%) vs 61 (63.5\%), $p=0.999]$. Most women who were sexually active before surgery stayed sexually active after surgery (55/63). Six patients $(9.5 \%)$ who were sexually inactive before surgery reported being sexually active after prolapse correction. Two of these six patients cited prolapse related issues as the reason for this change. For the remaining four patients, the source of the change is unknown. Five patients (7.9\%) were sexually active before surgery, but sexually inactive after surgery. Reasons for this change were loss of their partner $(n=2)$, no more interest in sex $(n=1)$, impossible to have sexual intercourse due to a painful sensation in the vagina since the operation $(n=1)$, and no reason given $(n=1)$.

\section{Discussion}

This prospective study shows a significant improvement in sexual function in patients with POP 1 year after robot-assisted prolapse surgery. Both physical and behavioralemotive domains of the PISQ-12 improved significantly. This suggests that amelioration of total sexual function was mainly caused by better physical and emotional scores after surgery. Due to the complicated nature of sexual functioning, ${ }^{17}$ it must be taken into 
account that more factors could have contributed to the changes observed here. Significantly fewer women avoided sex postoperatively compared with preoperatively due to vaginal bulging. However, the total number of patients suffering from dyspareunia did not significantly change. Postoperatively, fewer patients reported having dyspareunia always or mostly. De novo dyspareunia occurred in $4.7 \%$ of patients. No differences were seen in the total number of sexually active women before and after surgery, and there was no discernible trend in the reasons for changes in sexual activity. Additionally, the amount of improvement of sexual function in women $>60$ years appeared to be similar to that in younger women.

A more severe prolapse of the middle compartment (sPOPQ C) was found in sexually inactive women. This implies that severity of apical prolapse and sexual function are directly related, which is in line with the study of Ellerkmann et al. ${ }^{18}$ Another explanation could be that participants who are sexually active seek medical help at a less severe stage of prolapse. There was no difference in the use of vaginal estrogens between sexually active and inactive patients. Vaginal estrogens were only prescribed in case of complains (such as vaginal dryness or dyspareunia) and not as a preventive measure. Some research indicates that intravaginal estrogens may improve symptoms of vaginal atrophy in postmenopausal patients, but the evidence is not conclusive. ${ }^{19}$ Only a very small percentage of all patients in this study used vaginal estrogens, and therefore, we believe the influence on the results will be limited.

The robot-assisted technique may be of influence in the improvement of sexual function. Women with POP may have decreased vaginal wall sensitivity and surgical correction could possibly play a role in the restoration of clitoral and vaginal wall sensation. ${ }^{20,21}$ The robot-assisted approach is associated with improved performance of intracorporeal suturing compared with laparoscopic surgery. ${ }^{22}$ Precise suturing of the vaginal walls could benefit sexual function. A randomized controlled trial with adequate power is the only way to resolve this question. Only a few other studies with limited patients have described sexual function after using robot-assisted prolapse surgery. ${ }^{8-11}$ One study performed by Geller et al. ${ }^{8}$ investigated outcomes 1 year after RASC in 25 women with advanced POP and showed stable sexual function but no improvement after surgery. One randomized trial compared LSC with RASC and showed an improvement in total PISQ-12 scores for both groups. ${ }^{23}$ The number of sexually active patients postoperative was 14 and 15 in the two groups. Prior published articles after LSC have documented an improvement in sexual function, which is in line with our results. ${ }^{24-28}$ 
Strong points of this study are the prospective design, the large number of patients included, and the long median follow-up (15.3 months). Heterogeneity of the study group was minimized by standardized surgery and only evaluating patients after a RASC or RSHS. Patients who underwent concomitant vaginal surgery were excluded, as this may lead to vaginal narrowing and possibly dyspareunia. ${ }^{29}$ Anti-incontinence procedures were also eliminated. The latter is thought to compromise neural tissue in the vaginal wall, thereby affecting orgasmic response. ${ }^{30}$

Limitations of this study are that patients were treated in a tertiary referral hospital for pelvic floor dysfunction. Therefore, this population often had a history of pelvic floor disorders which could limit the generalizability of the study. Procedures were performed by surgeons experienced in open abdominal, conventional laparoscopic and robot-assisted pelvic prolapse procedures. This is important, because tensioning of the mesh is a crucial aspect of this procedure. Using too little or too much tension may result in either insufficient repair or postoperative pain or discomfort. ${ }^{16}$ Secondly, only one center was included in this study. Third, we did not conduct a randomized controlled trial comparing robot-assisted surgery with other techniques, which is a limitation. Finally, not all sexually active patients filled in the questionnaire, resulting in loss to follow-up. Although all patients received the questionnaire, some patients left it blank or only partly completed it. Possible reasons are negative feelings, such as shame regarding questions about their sexual life, or due to different cultural aspects.

Patients who underwent a subtotal hysterectomy concomitantly were not excluded, since this is an essential part of treating apical prolapse. A hysterectomy may affect sexual function negatively (absence of rhythmic uterine contractions during orgasms) or positively (resolving underlying uterine condition such as prolapse, menorrhagia). ${ }^{29}$ Zucchi et al. found an improvement of sexual function after removal of the uterus with prolapse surgery. ${ }^{31}$ We found no difference in pre- and postoperative sexual function between the two surgery types. However, our finding was based on subgroup analysis with a limited number of patients $(n=24$ and $n=18)$, making further research necessary. In spite of the worldwide increased use of robot-assisted prolapse surgery, ${ }^{32}$ data on sexual function after the robotic technique are limited. The results of this study contribute to a better knowledge of sexual dysfunction in patients suffering from POP treated with the robot-assisted approach. 


\section{Conclusion}

This prospective cohort study shows improvement in sexual function one year after robot-assisted apical prolapse surgery due to enhanced physical and emotional scores. The total number of sexually active women and women suffering from dyspareunia before and after surgery did not differ. The findings are important for proper counselling of patients prior to operative POP treatment.

\section{Commentary}

A commentary on 'Sexual function after robot-assisted prolapse surgery: a prospective study' by Debjyoti Karmakar was published in the International Urogynecology Journal (Commentary on "Sexual function after robot-assisted prolapse surgery". Int Urogyn J. 2018;29:921). 


\section{References}

1. Wu JM, Hundley AF, Fulton RG, Myers ER. Forecasting the prevalence of pelvic floor disorders in U.S. women: 2010 to 2050. Obstet Gynecol. 2009;114:1278-1283.

2. Novi JM, Jeronis S, Morgan MA, Arya LA. Sexual Function in women with pelvic organ prolapse compared to women without pelvic organ prolapse. J Urol. 2005;173:1669-1672.

3. 't Hoen LA, Utomo E, Steensma AB, Blok BFM, Korfage IJ. The pelvic organ prolapse/urinary incontinence sexual questionnaire (PISQ-12): validation of the Dutch version. Int Urogynecol J. 2015;26:1293-1303.

4. Freeman RM, Pantazis K, Thomson A, Frappell J, Bombieri L, Moran P, et al. A randomised controlled trial of abdominal vs. laparoscopic sacrocolpopexy for the treatment of posthysterectomy vaginal vault prolapse: LAS study. Int Urogynecol J. 2013;24:377-384.

5. Lane FE. Repair of posthysterectomy vaginal-vault prolapse. Obstet Gynecol. 1962;20:72-77.

6 Rosati M, Bramante S, Bracale U, Pignata G, Azioni G. Efficacy of laparoscopic sacrocervicopexy for apical support of pelvic organ prolapse. JSLS. 2013;17:235-244.

7. Sarlos D, Brandner S, Kots L, Gygax N, Schaer G. Laparoscopic sacrocolpopexy for uterine and posthysterectomy prolapse: anatomical results, quality of life and perioperative outcome-a prospective study with 101 cases. Int Urogynecol J. 2008;19:1415-1422.

8. Geller EJ, Parnell BA, Dunivan GC. Pelvic floor function before and after robotic sacrocolpopexy: one-year outcomes. J Minim Invasive Gynecol. 2011;18:322-327.

9. Geller EJ, Parnell BA, Dunivan GC. Robotic vs abdominal sacrocolpopexy: 44-month pelvic floor outcomes. Urology. 2012;79:532-536.

10. Gupta P, Payne J, Killinger KA, Ehlert M, Bartley J, Gilleran J, et al. Analysis of changes in sexual function in women undergoing pelvic organ prolapse repair with abdominal or vaginal approaches. Int Urogynecol J. 2016;27:1919-1924.

11. van Iersel JJ, de Witte CJ, Verheijen PM, Broeders IAMJ, Lenters E, Consten ECJ, et al. Robot-assisted sacrocolporectopexy for multicompartment prolapse of the pelvic floor: a prospective cohort study evaluating functional and sexual outcome. Dis Colon Rectum. 2016;59:968-974.

12. Swift S, Morris S, McKinnie V, Freeman R, Petri E, Scotti RJ, et al. Validation of a simplified technique for using the POPQ pelvic organ prolapse classification system. Int Urogynecol J. 2006;17615-620.

13. Parekh M, Swift S, Lemos N, Iskander M, Freeman B, Arunkalaivanan AS, et al. Mulitcenter interexaminer agreement trial for the validation of simplified POPQ system. Int Urogynecol J. 2011;22:645-650.

14. Rogers RG, Coates KW, Kammerer-Doak D, Khalsa S, Qualls C. A short form of the pelvic organ prolapse / urinary incontinence sexual questionnaire (PISQ-12 ). Int Urogynecol J. 2003;14:164168.

15. Diez-Itza I, Aizpitarte I, Becerro A. Risk factors for the recurrence of pelvic organ prolapse after vaginal surgery: a review at 5 years after surgery. Int Urogynecol J. 2007;18:1317-24.

16. Clifton MM, Pizarro-Berdichevsky J, Goldman HB. Robotic female pelvic floor reconstruction: a review. Urology. 2016;91:33-40.

17. Handa VL, Zyczynski HM, Brubaker L, Nygaard I, Janz NK, Richter HE, et al. Sexual function before and after sacrocolpopexy for pelvic organ prolapse. Am J Obstet Gynecol. 2007;197:629.e1-6. 
18. Ellerkmann RM, Cundiff GW, Melick CF, Nihira MA, Leffler K, Bent AE. Correlation of symptoms with location and severity of pelvic organ prolapse. Am J Obstet Gynecol. 2001;185:1332-8.

19. Lethaby A, Ayeleke RO, Roberts H. Local oestrogen for vaginal atrophy in postmenopausal women. Cochrane Database of Syst Rev. 2016;8;CD001500.

20. Gruenwald I, Mustafa S, Gartman I, Lowenstein L. Genital sensation in women with pelvic organ prolapse. Int Urogynecol J. 2015;26:981-984.

21. Lowenstein L, Mustafa-Mikhail S, Gartman I, Gruenwald I. The effect of pelvic organ prolapse repair on vaginal sensation. Int Urogynecol J. 2016;27:915-918.

22. Stefanidis D, Wang F, Korndorffer JR Jr, Dunne JB, Scott DJ. Robotic assistance improves intracorporeal suturing performance and safety in the operating room while decreasing operator workload. Surg Endosc. 2010;24:377-382.

23. Kenton K, Mueller ER, Tarney C, Bresee C, Anger JT. One-year outcomes after minimally invasive sacrocolpopexy. Female Pelvic Med Reconstr Surg. 2016;22:382-384.

24. Salamon CG, Lewis CM, Priestley J, Culligan PJ. Sexual function before and 1 year after laparoscopic Sacrocolpopexy. Female Pelvic Med Reconstr Surg. 2014;20:44-47.

25. Price N, Slack A, Jackson SR. Laparoscopic sacrocolpopexy: an observational study of functional and anatomical outcomes. Int Urogynecol J. 2011;22:77-82.

26. Perez T, Crochet P, Descargues G, Tribondeau P, Soffray F, Gadonneix P, et al. Laparoscopic sacrocolpopexy for management of pelvic organ prolapse enhances quality of life at one year: a prospective observational study. J Minim Invasive Gynecol. 2011;18:747-754.

27. Thibault F, Costa P, Thanigasalam R, Seni G, Brouzyine M, Cayzergues L, et al. Impact of laparoscopic sacrocolpopexy on symptoms, health-related quality of life and sexuality: a mediumterm analysis. BJU Int. 2013;112:1143-1149.

28. Liang S, Zhu L, Song X, Xu T, Sun Z, Lang J. Long-term outcomes of modified laparoscopic sacrocolpopexy for advanced pelvic organ prolapse: a 3-year prospective study. Menopause. 2016;23:765-770.

29. Rogers RG. Sexual function in women with pelvic floor disorders. Can Urol Assoc J. 2013;7:S199201.

30. Szell N, Komisaruk B, Goldstein S, Qu X, Shaw M, Goldstein I. A meta-analysis detailing overall sexual-function and orgasmic function in women undergoing midurethral sling surgery for stress incontinence. J Sex Med. 2017;S2050-1161.

31. Zucchi A, Costantini E, Mearini L, Fioretti F, Bini V, Porena M. Female sexual dysfunction in urogenital prolapse surgery: colposacropexy vs. hysterocolposacropexy. J Sex Med. 2008;5:139-45.

32. Carroll AW, Lamb E, Hill AJ, Gill EJ, Matthews CA. Surgical management of apical pelvic support defects : the impact of robotic technology. Int Urogynecol J. 2012;1183-1186. 




\section{Chapter 5}

Long-term anatomical and functional results of robot-assisted pelvic floor surgery for the management of multicompartment prolapse: a prospective study

Femke van Zanten, Emma M. van der Schans, Esther C.J. Consten Paul M. Verheijen, Egbert Lenters, Ivo A.M.J. Broeders Steven E. Schraffordt Koops 


\section{Abstract}

Background: Long-term data on robot-assisted sacrocolporectopexy for the treatment of multicompartment pelvic organ prolapse is scarce. With the rising prevalence of prolapse and increasing surgical repair, it is essential to evaluate long-term results.

Objective: To evaluate long-term functional and anatomic outcomes after sacrocolporectopexy.

Design: Prospective, observational cohort study.

Settings: Teaching hospital with tertiary referral function for patients with gynecological/rectal prolapse.

Patients: All patients undergoing robot-assisted sacrocolporectopexy in 2011-2012.

Intervention: Robot-assisted sacrocolporectopexy.

Main Outcome Measures: Primary outcome was anatomic cure rate after one and four years, defined as simplified pelvic organ prolapse quantification stage 1 vaginal apical prolapse and no external rectal prolapse or internal rectal prolapse present. Kaplan Meier Curves were used for determination of recurrence free intervals. Secondary outcomes were functional pelvic floor symptoms (symptoms of bulge, obstructed defecation, fecal incontinence, urogenital distress inventory) and quality of life (Pelvic Floor Impact Questionnaire).

Results: Fifty-three patients were included. After 12 and 48 months, recurrence free interval based on Kaplan Meier estimates were 100\% and 90\% respectively. In total there were ten recurrences: two apical and eight internal rectal prolapses. Symptoms of bulge ( $94 \%$ to $12 \% ; p<0.0005$ ), fecal incontinence ( $62 \%$ to $32 \%$; $p<0.0005$ ), obstructed defecation (59\% to $24 \% ; p=0.008)$ and median Pelvic Floor Impact Questionnaire scores (124 to $5 ; p=0.022$ ) improved significantly at final follow-up. Median urogenital distress inventory scores showed improvement after one year (30 to $13 ; p=0.021$ ).

Limitations: Observational, single center study with selective postoperative imaging.

Conclusions: Ninety percent of patients were recurrence free 48 months after robotassisted sacrocolporectopexy. Symptoms of vaginal bulge, quality of life, constipation and fecal incontinence improved significantly. However, a subgroup of patients showed persistent bowel complaints which underlies the complexity of multicompartment prolapse. 


\section{Introduction}

Pelvic organ prolapse (POP), including rectal prolapse (RP), is a condition mostly affecting middle-aged women. Depending on the type of prolapse, symptoms can include urinary or fecal incontinence, obstructed defecation, pelvic pain, symptoms of bulge, and sexual dysfunction. These symptoms greatly impair quality of life (QoL) and have a growing worldwide serious socio-economic burden due to the increasing prevalence of POP with aging population. ${ }^{1,2}$

Pelvic floor disorders can be found in the anterior, middle or posterior compartment. They are traditionally treated by the associated specialty, i.e. urology, gynecology or colorectal surgery. Multicompartment prolapses are commonly found as well with rates reported of $47 \%$ and higher. 3,4 These numbers are probably still underestimated as limited research has been performed. Therefore, single compartment prolapse should alert the possibility of prolapse in another part of the pelvis. After hysterectomy, the prevalence of multicompartment prolapse is high, as a hysterectomy leads to loss of pelvic support. ${ }^{5}$ Rectoceles often occur simultaneously with an internal rectal prolapse (IRP). ${ }^{6}$ Therefore, evaluation of patients with POP by a multidisciplinary team is crucial.

Currently, for vaginal apical prolapse, laparoscopic sacrocolpopexy (LSC) is one of the preferred treatments. ${ }^{7}$ In surgery for RP - i.e. external rectal prolapse (ERP), IRP with or without rectocele and enterocele - laparoscopic ventral mesh rectopexy (LVR) is widely accepted, although other types of surgical repair are being performed as well (e.g. resection or suture rectopexy and perineal approaches). ${ }^{8}$ Complex, multicompartment prolapses have been treated by combining sacrocolpopexy with ventral mesh rectopexy, which has shown to be safe without raised morbidity. ${ }^{6,9,10}$ This integrative treatment, performed laparoscopically or with robotic assistance, has improved functional outcomes on the short and mid-term. ${ }^{11,12,13}$ Long-term results are scarce though. ${ }^{14}$ Robotic assistance is rapidly increasing in pelvic floor surgery due to its advantages in complex maneuvers such as intracorporeal suturing deep in the narrow pelvis. ${ }^{15}$ The objective of this study was to assess short- and long-term outcome after robot-assisted laparoscopic sacrocolporectopexy (RSCR). 


\section{Materials \& Methods}

\section{Study design}

All consecutive patients undergoing RSCR in 2011 and 2012 for the treatment of female multicompartment pelvic organ prolapse were included. Patients were treated in our tertiary referral center for pelvic floor dysfunction. This prospective study was carried out in accordance with the ethical standards of the Central Committee on Research Involving Human Subjects (CCMO) and with the Declaration of Helsinki. For all patients informed consent was obtained.

\section{Patient evaluation}

Preoperatively all patients were evaluated by an urogynecologist and a colorectal surgeon. Proctoscopy and speculum examination were performed routinely. Genital prolapse was scored using the simplified Pelvic Organ Quantification (sPOPQ). SPOPQ scores prolapse by using the following landmarks: $\mathrm{Ba}$ ) anterior vaginal wall, $\mathrm{Bp}$ ) posterior vaginal wall, C) cervix/vaginal vault in post hysterectomy patients, D) posterior fornix. ${ }^{16}$ Stage 1 represents either no or minimal prolapse. Stage 2 represents descending of the most distal point of the prolapsed tissue one centimeter proximal until one centimeter distal to the hymnal remnants. Stage 3 and 4 are prolapse of landmarks beyond one centimeter distal to the hymnal remnants. Where the patient had a hysterectomy (e.g. no cervix), landmark ' $\mathrm{D}$ ' of the sPOPQ is by definition no longer described. Rectal prolapse was diagnosed based on a combination of physical examination and imaging.

All women underwent dynamic magnetic resonance imaging (dMRI) in supine position and/or a conventional defecogram preoperatively (Table 1). All patients were discussed in a multidisciplinary team (Figure 1) and patients were counselled. RSCR was considered if a combination was present of:

- an ERP or a high-grade IRP (a recto-anal intussusception) ${ }^{17} \pm$ rectocele or enterocele;

- gynecological middle compartment prolapse: vaginal vault prolapse/ uterine descensus (sPOPQ C $\geq$ stage 2);

- disabling multicompartment complaints not responding to conservative therapy (i.e. fecal incontinence, obstructed defecation, sensation of a vaginal bulge, micturition symptoms). 
Figure 1. Flowchart of diagnostic work-up

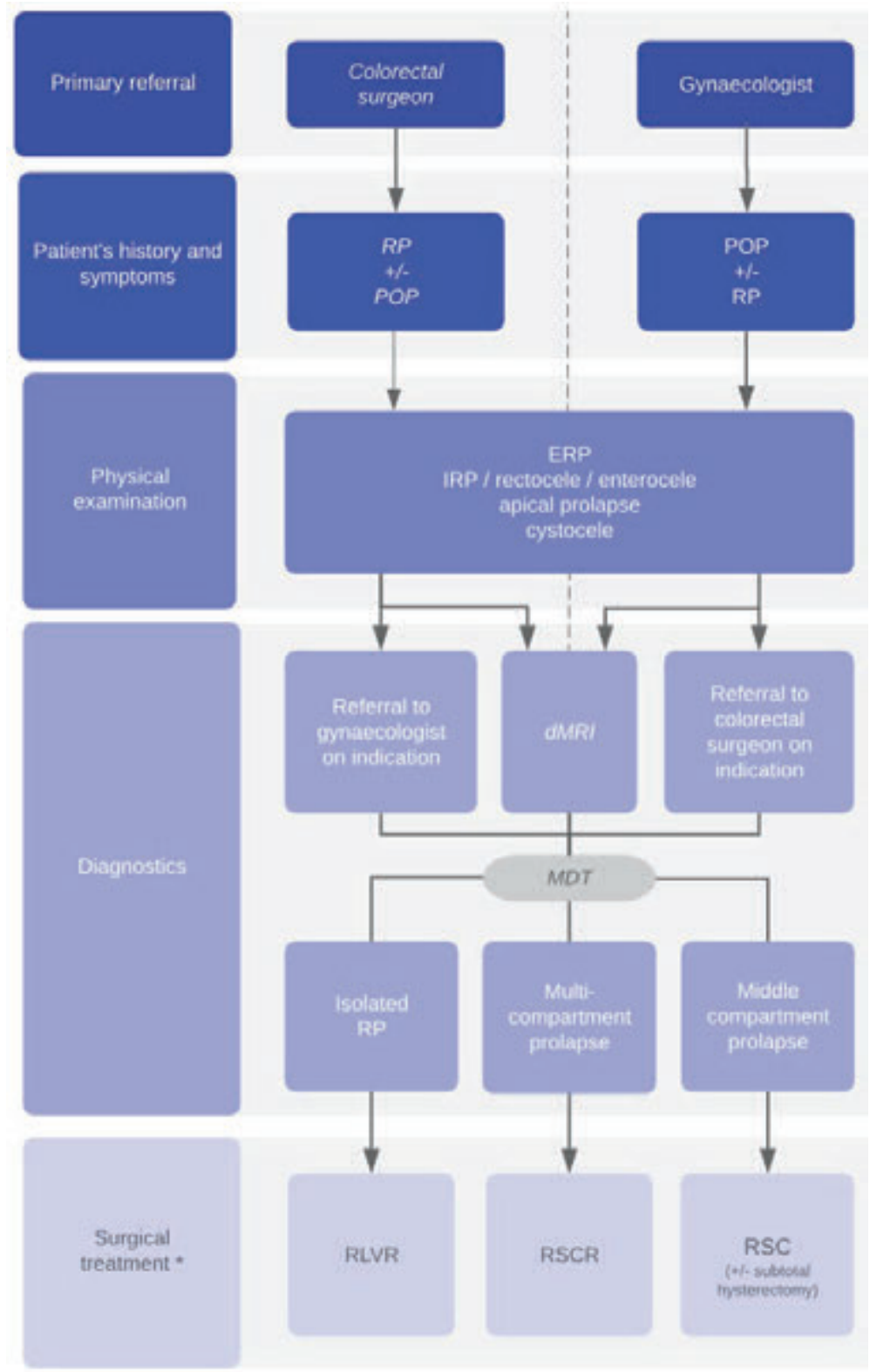

*Surgical treatment is offered after unsuccessful conservative treatment when no contra-indication for (minimal invasive) abdominal surgery exists. $d M R I$ dynamic magnetic resonance imaging ERP external rectal prolapse IRP internal rectal prolapse $M D T$ multidisciplinary team $P O P$ pelvic organ prolapse $R L V R$ robot-assisted laparoscopic ventral rectopexy $R P$ rectal prolapse $R S C$ robot-assisted laparoscopic sacrocolpopexy RSCR robot-assisted laparoscopic sacrocolporectopexy 
Functional complaints were assessed pre-and postoperatively with help of (validated) questionnaires:

- questions on bowel complaints of:

○ obstructed defecation following the Rome III criteria ${ }^{18}$

$\circ$ frequency and consistency of fecal incontinence. Fecal incontinence was then indexed according to the Browning \& Parks scale (B\&P). ${ }^{19}$ This scale includes four categories: grade 1 continent; grade 2 incontinent for gas; grade 3 incontinent for gas and liquid defecation; grade 4 incontinent for gas, liquid and solid defecation. Fecal incontinence was defined as B\&P grade 3-4 with complaints at least once a month;

- questions on symptoms of vaginal prolapse (sensation of and/or seeing vaginal bulge);

- urogenital distress inventory (UDI-6) for micturition symptoms. The UDI-6 consists of three subscales: irritative, stress incontinence and obstructive symptoms (range of 0-100);20

- Pelvic Floor Impact Questionnaire (PFIQ-7) for pelvic floor related quality of life (QoL). A higher score indicates a higher impact of complaints on daily life (range $0-300) \cdot{ }^{21}$

\section{Outcome-measurements and follow-up}

Primary outcome was anatomic cure rate, defined as SPOPQ C stage 1 (middle compartment), with no ERP or high-grade IRP present (posterior compartment). In patients with POP related complaints, postoperative imaging was performed additionally. Recurrence was scored in case patients did not meet the criteria of anatomic cure. Kaplan-Meier estimates were used to describe the recurrence free interval. Secondary outcomes were functional symptoms and pelvic floor related QoL assessed by the questionnaire. Postoperative re-interventions, including pessary use, were scored.

Routine post-surgery consultations were set at six weeks, one year and four years. Patients were considered lost to follow-up if there was no postoperative consultation available and no questionnaire. 


\section{Surgical technique}

The technique and materials used have been described in detail previously. ${ }^{11}$ In summary, after docking the da Vinci Si (Intuitive Surgical, Inc., Sunnyvale, CA, USA) the repair was started by performing a ventral mesh rectopexy according to D'Hoore et al.22 A polypropylene mesh (sized $3 \mathrm{~cm} \times 20 \mathrm{~cm}$ ) was used (Prolene, Ethicon Inc., Johnsen \& Johnson, Hamburg, Germany, weight 80$85 \mathrm{~g} / \mathrm{m}^{2}$ ). This was followed by a sacrocolpopexy and, if the uterus was in situ, a supracervical hysterectomy was performed, followed by a cervicosacrocolpopexy. Then dissection of the bladder from the anterior vaginal wall took place and a second piece of polypropylene mesh was sutured to the anterior vaginal wall and cervix/vaginal apex with non-absorbable sutures. The posterior vaginal wall was sutured to the rectopexy mesh. Both meshes were then connected to the cervix/vaginal apex into a Y-shape. Finally, the peritoneum was closed to cover the mesh. The rectopexy was performed by a colorectal surgeon; the sacrocolpopexy /supracervical hysterectomy with cervicopexy by an urogynecologist. Our multidisciplinary team holds three colorectal and two urogynecologists.

\section{Statistical analysis}

Statistical analysis was performed using SPSS v. 22.0 (IBM Corp., Armonk, NY, USA). A pvalue of $<0.05$ was considered significant. Data were presented as mean \pm SD and median and range for parametric and non-parametric distributed continues values respectively. Number and percentages were used for nominal and categorical values. Independent-Samples T-test, Mann-Whitney $\mathrm{U}$ test, Chi-square test were used to compare data for mean, median and nominal values respectively. Kaplan Meier curves were made to describe time till recurrence.

\section{Results}

\section{Patients}

Fifty-three patients were included. Baseline characteristics of the patients are depicted in Table 1. Mean age was $62.0 \pm 12.1$. Forty-three percent of patients had a history of previous POP or anti-incontinence surgery. Median time to follow-up was 14.1 months (range 5.5-35.1) for the one-year postoperative consultation and 48.2 months (range 37.7-62.1) for the final consultation. 
Table 1. Baseline demographics and perioperative data $\mathrm{N}=53$

\begin{tabular}{|c|c|c|c|}
\hline \multicolumn{2}{|l|}{ Baseline demographics } & \multicolumn{2}{|l|}{ Perioperative data } \\
\hline Age (years) & $62.0 \pm 12.1$ & Mean blood loss (ml) & 5362 \\
\hline BMI & $25.8 \pm 3.4$ & Mean total surgery time (min) & $183 \pm 35$ \\
\hline Previous pelvic floor surgery & $23(43.4)$ & Concomitant surgery & \\
\hline Hysterectomy for POP & $19(35.8)$ & Subtotal hysterectomy & $28(52.8)^{c}$ \\
\hline Hysterectomy for other indications & $6(11.3)$ & TVT & $3(5.7)$ \\
\hline Ant. Colporrhaphy & $16(30.2)$ & Oophorectomy ${ }^{\mathrm{d}}$ & $1(1.9)$ \\
\hline Post. Colporrhaphy & $14(26.4)$ & Ant. Colporrhaphy & $2(3.8)$ \\
\hline Minimal-invasive VMR & $1(1.9)$ & Post. Colporrhaphy & $0(0.0)$ \\
\hline TVT & $4(7.5)$ & Other & $1(1.9)$ \\
\hline Other & $6(11.3)$ & & \\
\hline Intra-abdominal surgery ${ }^{a}$ & $31(58.5)$ & Intraoperative complications & $3(5.7)$ \\
\hline Postmenopausal & $42(79.2)$ & Conversion & $1(1.8)$ \\
\hline Parity & $3(1-6)$ & $\begin{array}{l}\text { Mean length of hospital stay } \\
\text { (days) }\end{array}$ & $3.2 \pm 1.1$ \\
\hline Sphincter rupture labour & $1(1.9)$ & & \\
\hline Episiotomy labour & $22(41.5)$ & & \\
\hline \multicolumn{4}{|l|}{ ASA score } \\
\hline 1 & $17(32.1)$ & & \\
\hline 2 & $33(62.3)$ & & \\
\hline 3 & $3(5.7)$ & & \\
\hline Sexually active & $27(50.9)$ & & \\
\hline Smoking (active) & $12(22.6)$ & & \\
\hline \multicolumn{4}{|l|}{ Preoperative imaging } \\
\hline dMRI & $49(92.5)$ & & \\
\hline Conventional defecogram & $1(1.9)^{\mathrm{b}}$ & & \\
\hline both & $3(5.7)$ & & \\
\hline \multicolumn{4}{|c|}{ 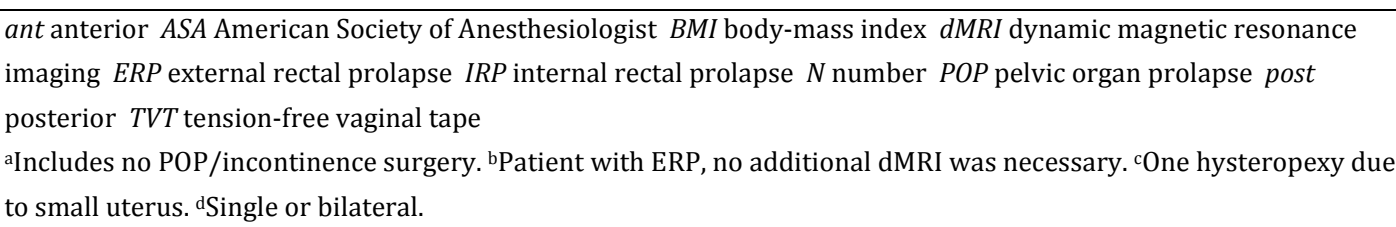 } \\
\hline
\end{tabular}

Forty-eight patients (91\%) were examined after one year and 42 (79\%) after four years (Figure 2). At final follow-up, two patients declined prolapse related consultations because they received a stoma (one due to therapy-resistant fecal incontinence as a result of severe sphincter dysfunction and one in the setting of treatment for colorectal malignancy). One patient was lost to follow-up for unknown reasons. One patient 
declined physical examination since she experienced no complaints. Seven patients solely responded by questionnaire.

Figure 2. Flowchart of included patients

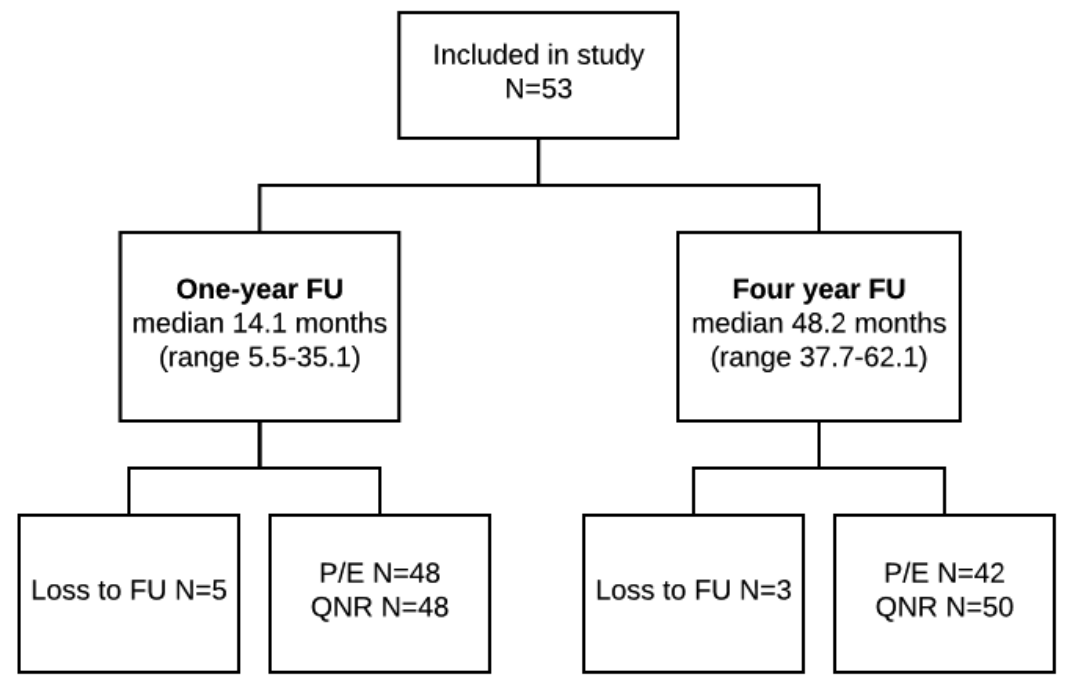

$F U$ follow-up $N$ number $P / E$ physical examination $Q N R$ questionnaire

\section{Anatomic results and post-interventions}

The mean values of SPOPQ Ba, Bp and C improved significantly ( $p<0.0005$; Table 2). Two patients developed an asymptomatic stage 2 recurrence of the apical compartment. There were no patients with a recurrent full-thickness RP (Table 2). In thirteen patients with suspected POP complaints an additional dMRI was performed: recurrent highgrade IRP and/or rectocele was diagnosed in eight patients (Table 2). One redorectopexy and one Delormes procedure was performed. In other symptomatic patients no imaging was performed due to minimal complaints or other pathologies causing the symptoms (e.g. dysfunctional sphincter, stress urinary incontinence).

With aid of Kaplan Meier analyses, estimated recurrence free percentages were calculated: $100 \%$ after 12 months, 94\% after 24 months, 92\% after 36 months and 90\% after 48 months (Figure 3). After 60 months, this percentage lowered till 56\%, but showed broad $95 \%$ CI. 
Seven patients were treated for hemorrhoids and two for sphincter dysfunction. Due to micturition problems seven patients received further treatment (Tension-free vaginal tape $n=5$, other $n=2$ ). At final follow-up, one patient was diagnosed with an asymptomatic vaginal mesh exposure (1.9\%). This was treated with vaginal estrogen cream.

Table 2. Pre- and postoperative Pelvic Organ Prolapse

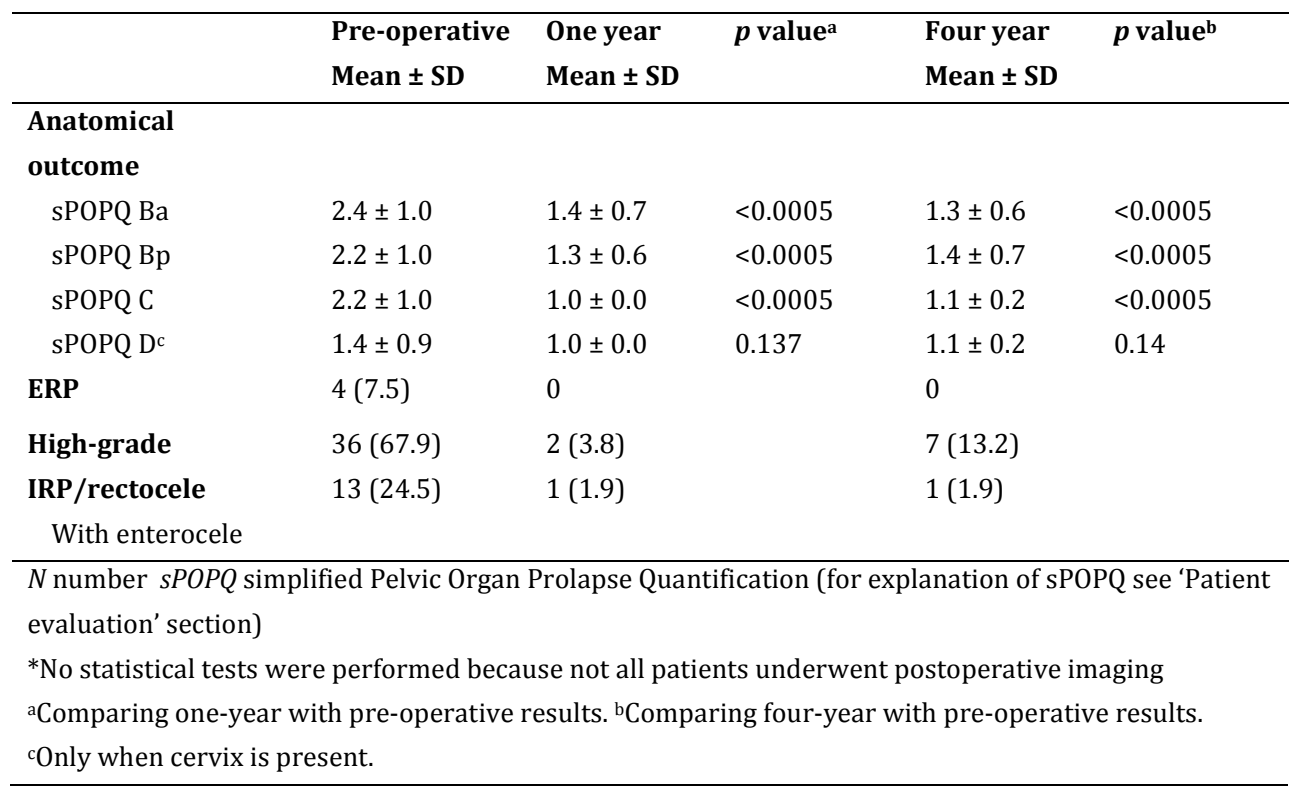


Figure 3. Kaplan-Meier curve for recurrence after RSCR

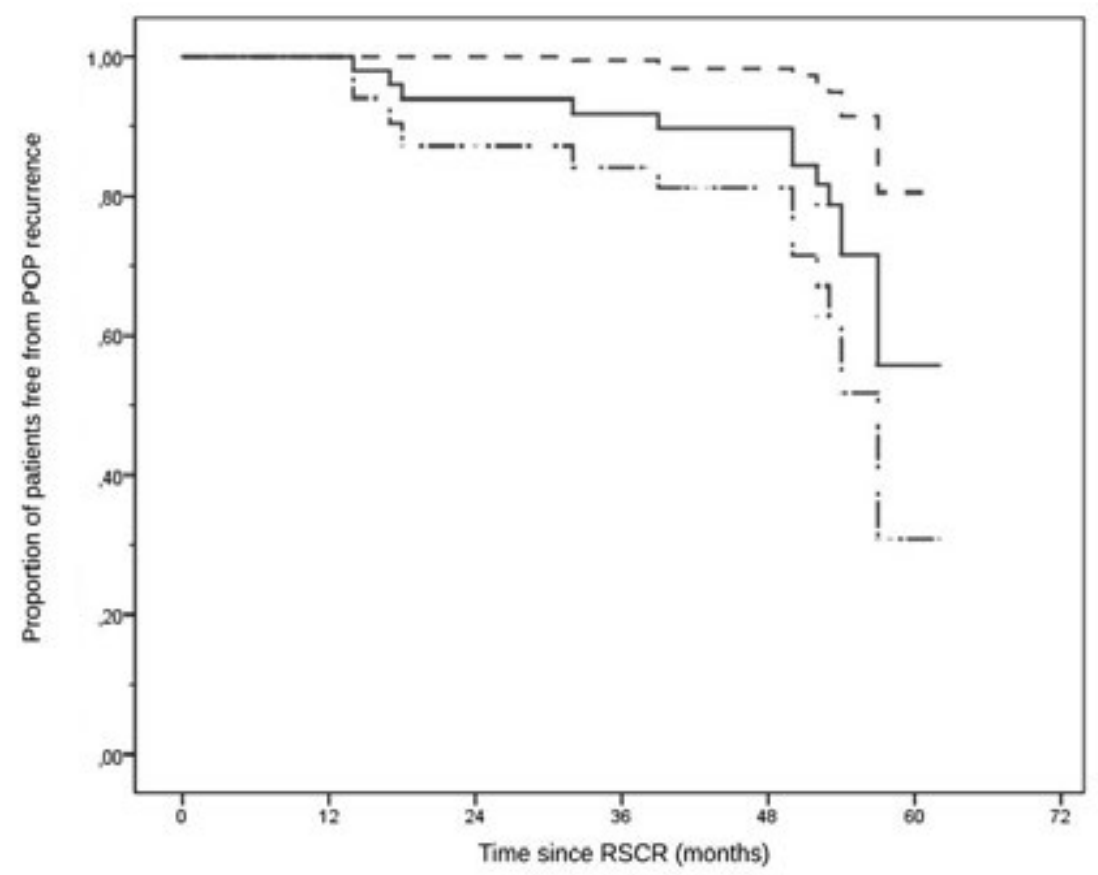

\begin{tabular}{l|l|l|l|l|l|l|}
\hline No. at risk & 53 & 49 & 45 & 44 & 23 & 1 \\
\hline Mean RFS & & 1.0 & 0.94 & 0.92 & 0.90 & 0.56 \\
\hline $\mathbf{9 5 \%} \mathrm{CI}$ & & $1.0-1.0$ & $0.87-1.0$ & $0.84-1.0$ & $0.81-0.99$ & $0.30-0.81$
\end{tabular}

Kaplan-Meier curve for recurrence after RSCR (straight line) The dotted lines represent the upper and lower 95\% CI limits.

$C I$ confidence interval $P O P$ pelvic organ prolapse $R F S$ recurrence free survival $R S C R$ robot-assisted laparoscopic sacrocolporectopexy

\section{Functional results}

Functional results are presented in Table 3. Symptoms of vaginal bulge diminished significantly from $94 \%$ prior to surgery to $12 \%$ at final follow-up. After one year UDI-6 total scores improved significantly $(p=0.021)$, especially in respect to obstructive complaints (Table 3). Before surgery 31 patients (59\%) and 33 patients $(62 \%)$ had complaints of obstructed defecation and fecal incontinence respectively. At last followup symptoms significantly improved in $63 \%$ of patients with obstructed defecation and $45 \%$ of patients with fecal incontinence prior to surgery. Five patients experienced newonset obstructed defecation: two patients after one year (4.2\%) and three patients after four years $(6.0 \%)$. There were no cases of de-novo fecal incontinence. 
The PFIQ-7 questionnaire on quality of life showed a low preoperative response rate of only $25 \%$. However, postoperatively the response rate was $79 \%$. Significant improvement of median PFIQ-7 scores were seen. Mean improvement of scores was 54.7 and 71.0 points after one and four years respectively.

Table 3. Functional results

\begin{tabular}{|c|c|c|c|c|c|}
\hline & $\begin{array}{l}\text { Preoperative } \\
\mathrm{N}=53\end{array}$ & $\begin{array}{l}\text { FU } 14 \mathrm{mo} . \\
\mathrm{n}=48\end{array}$ & $p$ value & $\begin{array}{l}\text { FU } 48 \mathrm{mo} . \\
\mathrm{n}=50\end{array}$ & $p$ value \\
\hline Symptoms of bulge & $50(94.3)$ & $7(14.6)$ & $<0.0005$ & $6(12.0)$ & $<0.0005$ \\
\hline UDI-6 total $(0-100)$ & $30.0(0-80)$ & $13.3(0-60)$ & 0.02 & $33.3(0-83)$ & 0.18 \\
\hline $\begin{array}{l}\text { Irritative symptoms } \\
(0-100)\end{array}$ & $33.3(0-100)$ & $16.7(0-83)$ & 0.11 & $33.3(0-100)$ & 0.01 \\
\hline Stress symptoms $(0-100)$ & $33.3(0-100)$ & $33.3(0-100)$ & 0.25 & $33.3(0-83)$ & 0.31 \\
\hline $\begin{array}{l}\text { Obstructive/discomfort } \\
(0-100)\end{array}$ & $33.3(0-100)$ & $16.7(0-67)$ & 0.003 & $16.7(0-100)$ & 0.18 \\
\hline Rome III ODS present & $31(58.5)$ & $13(27.1)$ & 0.001 & $12(24.0)$ & 0.008 \\
\hline Fecal incontinence & $33(62.3)$ & $19(39.6)$ & 0.02 & $16(32.0)$ & $<0.0005$ \\
\hline $\mathrm{B} \& \mathrm{P}$ grade 3 & $17(32.1)$ & $8(16.7)$ & & $4(8.0)$ & \\
\hline B\&P grade 4 & $16(30.2)$ & $11(22.9)$ & & $12(24.0)$ & \\
\hline \multicolumn{6}{|l|}{ PFIQ-7 (0-300) } \\
\hline Urinary impact (0-100) & $123.8(0-266.7)$ & $23.8(0-200.0)$ & 0.04 & $5.0(0-226.1)$ & 0.02 \\
\hline Colorectal-anal impact & $35.8(0-90.5)$ & $0(0-81.0)$ & 0.24 & $1.0(0-76.2)$ & 0.04 \\
\hline$(0-100)$ & $33.3(0-100)$ & $5.2(0-76.2)$ & 0.03 & $4.0(0-77.7)$ & 0.07 \\
\hline POP impact (0-100) & $28.6(0-85.7)$ & $0(0-66.6)$ & 0.08 & $0(0-72.2)$ & 0.008 \\
\hline \multicolumn{6}{|c|}{ Data presented as median (range) or N (\%). } \\
\hline $\begin{array}{l}B \& P \text { Browning and Parks } M \\
\text { pelvic floor impact question }\end{array}$ & $\begin{array}{l}\text { onths } N A \text { not al } \\
\text { e } P O P \text { pelvic or }\end{array}$ & $\begin{array}{l}\text { licable } O D S \text { obs } \\
\text { n prolapse } U D I\end{array}$ & icted def & tion syndrom & PFIQ \\
\hline
\end{tabular}

\section{Discussion}

Pelvic organ support and its relation to pelvic floor function and dysfunction is complex and still incompletely understood. It is thought that prolapse of each compartment of the pelvic floor shares a common pathophysiologic pathway, which is supported by the high prevalence of multicompartment prolapse. ${ }^{3,4}$ Acknowledgement of the existence of more than one prolapsed organ is essential and has changed surgical treatment to a multidisciplinary setting over the recent years, with improved patient outcome on the short- and mid-term view. ${ }^{11,12,13}$ This current study is the first to report on longer follow-up after RSCR. 
After 12 and 48 months, recurrence free interval based on Kaplan Meier estimates was $100 \%$ and $90 \%$ respectively. In total ten recurrent prolapses were seen, mainly of the posterior compartment. Two of these patients needed a re-intervention to treat recurrent posterior prolapse. In literature, the majority of studies quote recurrence rates between 0 and $6.9 \%$ for sacrocolporectopexy with a median follow-up between 12 and 54 months. ${ }^{11,13,14,23}$ These studies had either a shorter follow-up period ${ }^{11,13}$, high loss to follow-up ${ }^{23}$ or only postoperative evaluation with questionnaires. ${ }^{23}$ Furthermore, heterogeneity in definitions and outcome measurements was high. Since symptoms of bulge are associated with prolapsing of vaginal tissue beyond the hymnal remnants, we used the cut-off value of SPOPQ stage 2 or more as clinically relevant. ${ }^{24}$ Jallad et al. who retrospectively scored recurrence of both vaginal prolapse as ERP (but no IRP), and used a more similar definition of recurrence to ours, found recurrence rates of $20.3 \%$ [8.5\% vaginal prolapse, 13.5\% RP (median follow-up: 17 months)] after sacrocolporectopexy. ${ }^{12}$ We found similar rates with considerably longer follow-up. Our RP rates were higher, but did include IRP. A large study of 919 patients undergoing LVR for isolated RP, showed a 10-year recurrence rate of $8.2 \%(n=242)$ in ERP patients and $14.2 \%(n=677)$ for patients initially diagnosed with IRP. ${ }^{8}$ The slightly higher RP recurrence found in our cohort underlines the complexity and severity of multicompartment POP. Also our results of the Kaplan-Meier curve after 48 months are less reliable because of broad 95\% confidence intervals. We did find more cases of recurrent POP after 48 months implying the need for research with longer follow-up.

Relief of functional complaints and improved QoL are outcomes most important for the patient. We have earlier reported our one-year results on safety and functional outcome after RSCR of another comparable series of patients. ${ }^{11}$ With the current study we focused specifically on the sustainability of this surgery. To our knowledge, this is the second study reporting on long-term minimal-invasive results and the first on long term results after robotic surgery for multicompartment prolapse.

At final follow-up, symptoms of obstructed defecation and fecal incontinence were resolved in $63 \%$ and $45 \%$ of our patients respectively. Although this is a significant improvement, there still remains a fair amount of patients with persisting defecation problems. In the existing literature on POP a wide range of definitions for (improved) obstructed defecation and (improved) fecal incontinence are used which makes comparison complicated. Slawik et al., the only study reporting on long-term outcome after SCR (median follow-up 54 months) found a higher improvement of bowel symptoms: $80 \%$ of patients with resolved obstructed defecation (definition unclear) and 
91\% with improved fecal incontinence (Wexner incontinence score). ${ }^{14}$ These higher success rates could possibly be explained by the different definitions used. Also, their series of patients had a higher percentage of ERP at baseline (55\% versus $7.5 \%$ ), and only 31\% (versus 59\%) had preoperative obstructed defecation. No results of complaints of the other compartments were reported. Silvis et al. also used B\&P scale for fecal incontinence. When using the same definition of cured symptoms, a similar curation rate of $43 \%$ was found in their series of 25 patients 12 months after open SCR. ${ }^{25}$

More long-term results are reported in literature on single compartment prolapse. After minimal invasive ventral mesh rectopexy for prolapse of the posterior compartment, wide ranges of improved fecal incontinence and obstructive defecation are reported of $20-92 \%$ and $45-93 \%$ respectively. ${ }^{6}$ Here, a wide range of definitions was used as well. Results from our own pelvic floor clinic after ventral mesh rectopexy in large series of patients showed cure-rates of obstructed defecation and fecal incontinence between 76$79 \%$ and $64-77 \%$ respectively after mid- to long-term follow-up. ${ }^{8,26}$ The lower curerates found in this study again underlines the more complex POP in multicompartment patients. Also, the aetiology of functional bowel complaints can be multifactorial and the relation between anatomic abnormalities and symptoms is complex and not linear. This is supported by the difference found in restored anatomy and cured symptoms. Patients should be counselled for this.

RSCR positively influenced total UDI-6 scores on micturition after one year, mainly based on better obstructive scores. However, no difference was seen after four years. Increase of age could have been of influence on urge and stress incontinence symptoms. This emphasizes the complexity of treating multicompartment pelvic floor disorders once more. While providing apical support with sacrocolpopexy, support of the anterior compartment may prove more challenging. ${ }^{27}$

QoL improved significantly. Mean PFIQ-7 scores improved with 71 points at long-term evaluation. Utomo et al. ${ }^{21}$ suggested a change in PFIQ-7 of at least 31.8 points as clinically relevant. Missing data on preoperative PFIQ-7 values were high but are thought to be randomly missing. However, the conclusions drawn from these data should be drawn with cautiousness. 
A limitation of this study is that there was no control group. Furthermore, the single center nature of this study limited generalizability. Another limitation is that postoperative dMRI was performed only in patients with suspected recurrent POP. However, asymptomatic anatomic recurrences are, in our opinion, not clinically relevant.

We believe that patients' history could have been of influence on the results. Patients who underwent a previous hysterectomy for prolapse are more prone to have weak connective tissue and are more at risk for recurrence. However, this study was underpowered to look for risk factors for recurrence.

Strong points of this study are the long follow-up period with minimal loss to follow-up and the prospective design. Severity of functional complaints and findings on dMRI are poorly correlated. ${ }^{28}$ Focus on functional outcome was therefore another strength. Dichotomized cut-off points for obstructed defecation and fecal incontinence were used. We suggest future studies to use (patient reported) outcome measures for bowel symptoms with more than two gradations such as the Obstructed Defecation Score according to Altomare for obstructed defecation and the Fecal Incontinence Severity Index for fecal incontinence.

Multidisciplinary assessment is essential and careful preoperative evaluation with a tailored approach for individual patients should be made. This leads to more efficient treatment with single recuperation periods for the patient, and possibly reduces healthcare costs. ${ }^{29}$ This study emphasizes the need of multidisciplinary treatment for pelvic floor disorders. The results we presented are promising and could aid surgeons in treating complex multicompartment prolapse.

In conclusion, $90 \%$ of patients were recurrence free 48 months after RSCR based on physical examination and postoperative imaging. Symptoms of vaginal bulge, quality of life, obstructed defecation and fecal incontinence improved significantly. However, a substantial part of patients experienced persistent complaints of obstructed defecation and/or fecal incontinence. This underlies the complexity of treating multicompartment POP. 


\section{References}

1. Wu JM, Hundley AF, Fulton RG, Myers ER. Forecasting the prevalence of pelvic floor disorders in U.S. women 2010-2050. Obstetrics and Gynecology. 2009;114:1278-1283.

2. Wu JM, Kawasaki A, Hundley AF, Dieter AA, Myers ER, Sung VW. Predicting the number of women who will undergo incontinence and prolapse surgery, 2010-2050. Am J Obstet Gynecol. 2011;205:230.e1-230.e5.

3. Olsen AL, Smith VJ, Bergstrom JO, Colling YC, Clark AL. Epidemiology of surgically managed pelvic organ prolapse and urinary incontinence. Obstetrics \& Gynecology. 1997;89: 501-506.

4. Guzman Rojas R, Kamisan Atan I, Shek KL, Dietz HP. The prevalence of abnormal posterior compartment anatomy and its association with obstructed defecation symptoms in urogynecological patients. Int Urogynecol J. 2016;27:939-44.

5. Elneil S. Complex pelvic floor failure and associated problems. Best Pract Res Clin Gastroenterol. 2009;23:555-573.

6. van Iersel JJ, Paulides TJC, Verheijen PM, Lumley JW, Broeders IAMJ, Consten ECJ. Current status of laparoscopic and robotic ventral mesh rectopexy for external and internal rectal prolapse. World J Gastroenterol 2016;22:4977-4987.

7. Paraiso MFR, Jelovsek JE, Frick A, Chen CCG, Barber MD. Laparoscopic compared with robotic sacrocolpopexy for vaginal prolapse: a randomized controlled trial. Obstet Gynecol. 2011;118:1005-1013.

8. Consten ECJ, van Iersel JJ, Verheijen PM, Broeders IAMJ, Wolthuis AM, D'Hoore A. Long-term outcome after laparoscopic ventral mesh rectopexy: an observational study of 919 consecutive patients. Ann Surg. 2015;262:742-748.

9. Geltzeiler CB, Birnbaum EH, Silviera ML, Mutch MG, Vetter J, Wise PE, et al. Combined rectopexy and sacrocolpopexy is safe for correction of pelvic organ prolapse. Int J of Colorectal Dis 2018;33:1453-1459.

10. Weinberg D, Qeadan F, McKee R, Rogers RG, Komesu YM. Safety of laparoscopic sacrocolpopexy with concurrent rectopexy: peri-operative morbidity in a nationwide cohort. Int Urogynecol J. 2019;30:385-392.

11. van Iersel JJ, de Witte CJ, Verheijen PM, Broeders IAMJ, Lenters E, Consten ECJ, et al. Robot-assisted sacrocolporectopexy for multicompartment prolapse of the pelvic floor: a prospective cohort study evaluating functional and sexual outcome. Dis Colon Rectum. 2016;59:968-974.

12. Jallad K, Ridgeway B, Paraiso MFR, Gurland B, Unger CA. Long-term outcomes after ventral rectopexy with sacrocolpo- or hysteropexy for the treatment of concurrent rectal and pelvic organ prolapse. Female Pelvic Med Reconstr Surg 2018;24: 336-340.

13. Lim M, Sagar, PM, Gonsalves S, Thekkinkattil D, Landon C. Surgical management of pelvic organ prolapse in females: functional outcome of mesh sacrocolpopexy and rectopexy as a combined procedure. Dis Colon Rectum. 2007;50: 1412-1421.

14. Slawik S, Soulsby R, Carter H, Payne H, Dixon AR. Laparoscopic ventral recopexy, posterior colporrhaphy and vaginal sacrocolpopexy for the treatment of recto-genital prolapse and mechanic outlet obstruction. Colorectal Dis. 2008;10:138-143. 
15. Stefanidis D, Wang F, Korndorffer Jr. JR, Dunne JB, Scott DJ. Robotic assistance improves intracorporeal suturing performance and safety in the operating room while decreasing operator workload. Surg Endosc. 2010;24377-382.

16. Swift S, Morris S, McKinnie V, Freeman R, Petri E, Scotti RJ, et al. Validation of a simplified technique for using the $\mathrm{POPQ}$ pelvic organ prolapse classification system. Int Urogynecol J. 2006;17:615-620.

17. Wijffels NA, Collinson R, Cunningham C, Lindsey I. What is the natural history of internal rectal prolapse? Colorectal Dis 2010;12:822-30.

18. Drossman DA. The Functional Gastrointestinal Disorders and the Rome III Process. Gastroenterology. 2006;130:1377-1390.

19. Browning GG, Parks AG. Postanal repair for neuropathic faecal incontinence: correlation of clinical result and anal canal pressures. Br J Surg. 1983;70:101-4.

20. Utomo E, Korfage IJ, Wildhagen MF, Steensma AB, Bangma CH, Blok BF. Validation of the Urogenital Distress Inventory (UDI-6) and Incontinence Impact Questionnaire (IIQ-7) in a Dutch population. Neurourol Urodyn. 2015;34:24-31.

21. Utomo E, Blok BF, Steensma AB, Korfage IJ. Validation of the Pelvic Floor Distress Inventory (PFDI20) and Pelvic Floor Impact Questionnaire (PFIQ-7) in a Dutch population. Int Urogynecol J. 2014;25:531-544.

22. D’Hoore A, Penninckx F. Laparoscopic ventral recto(colpo)pexy for rectal prolapse: surgical technique and outcome for 109 patients. Surg Endosc. 2006;20:1919-1923.

23. Watadani U, Vogler S, Warshaw JS, Sueda T, Lowry AC, Madoff RD, et al. Sacrocolpopexy with rectopexy for pelvic floor prolapse improves bowel function and quality of life. Dis Colon Rectum 2013;56:1415-1422.

24. Barber MD, Brubaker L, Nygaard I, Wheeler TL, Schaffer J, Chen Z, et al. Defining success after surgery for pelvic organ prolapse. Obstet Gynecol 2009;114;600-609.

25. Silvis R, Gooszen HG, Kahraman T, Groenendijk AG, Lock MTWT, Italiaander MV, et al. Novel approach to combined defaecation and micturition disorders with rectovaginovesicopexy. $\mathrm{Br} \mathrm{J}$ Surg. 1998;85(6):813-817.

26. van Iersel JJ, Formijne Jonkers HA, Paulides TJC, Verheijen PM, Draaisma WA, Consten ECJ, et al. Robot-assisted ventral mesh rectopexy for rectal prolapse: a 5-year experience at a tertiary referral center. Dis Colon Rectum. 2017;60:1215-1223.

27. Wong V, Guzman Rojas R, Shek KL, Chou D, Moore KH, Dietz HP. Laparoscopic sacrocolpopexy: how low does the mesh go? Ultrasound Obstet Gynecol 2017;49:404-408.

28. Ramage L, Georgiou P, Qiu S, McLean P, Khan N, Kontnvounisios C, et al. Can we correlate pelvic floor dysfunction severity on MR defecography with patient-reported symptom severity? Updates Surg. 2018;70:467-476.

29. Kapoor DS, Sultan AH, Thakar R, Abulafi MA, Swift RI, Ness W. Management of complex pelvic floor disorders in a multidisciplinary pelvic floor clinic. Colorectal Dis. 2008;10:118-23. 



\section{Chapter 6}

Robot-assisted sacrocolpopexy: not only for vaginal vault suspension? An observational cohort study

Femke van Zanten, Egbert Lenters, Ivo A.M.J. Broeders, Steven E. Schraffordt Koops 


\section{Abstract}

Objective: Surgery for pelvic organ prolapse (POP) has high recurrence rates. Longterm anatomic and patient reported outcomes after pelvic floor repair are therefore required.

Design: Observational, prospective cohort study.

Setting: Teaching hospital with tertiary referral function for patients with POP.

Population: All patients undergoing robot-assisted sacrocolpopexy (RASC) or supracervical hysterectomy with sacrocervicopexy (RSHS) for symptomatic POP [simplified POP Quantification (sPOPQ) stage $\geq 2$ ].

Methods: Follow-up visits with SPOPQ evaluations were planned 5 years after surgery. Patients received pre- and postoperative questionnaires reporting symptoms of vaginal bulge, Urogenital Distress Inventory (UDI-6), and Pelvic Floor Impact Questionnaire (PFIQ-7).

Main Outcome Measures: Primary outcome was anatomic cure (sPOPQ stage 1) for all vaginal compartments. Secondary outcomes were patient reported outcomes.

Results: Seventy-seven patients were included. Sixty-one patients (79\%) were evaluated after 50 months (physical examination $n=51$ ). Median SPOPQ stages improved significantly in all four landmarks. Thirty patients (59\%) were completely recurrence free and $96 \%$ of patients had no apical recurrence. Most recurrences were asymptomatic cystoceles (20\%). Two patients (3.0\%) received a re-intervention due to prolapse. On the patient self-reporting questionnaires, symptoms of bulge (95\% vs $15 \%$ $p<0.0005$ ), median UDI-6 scores (26.7 vs $22.2 ; p=0.048$ ) and median PFIQ-7 (60.0 vs 0; $p=0.008$ ) scores improved significantly from pre to post-operative visit.

Conclusions: RASC and RSHS show sustainable results in the treatment of prolapse. Symptoms of bulge, urinary symptoms and quality of life improved substantially 50 months postoperatively. Patients should be counselled about the risk of an anterior wall recurrence and small chance for recurrent surgery. 


\section{Introduction}

About 1 in 6 women (11-19\%) undergo a surgical pelvic organ prolapse (POP) correction due to prolapse or urinary incontinence related complaints. ${ }^{1}$ After primary surgical repair of female POP, high recurrence rates are found.2,3 Vaginal vault prolapse is common and specifically recurrences in the anterior compartment are a recognized long standing problem. ${ }^{2-4}$ Determination of long-term outcomes for the patient after prolapse surgery is therefore essential. Open abdominal sacrocolpopexy (ASC) has been shown to have lower recurrent vault prolapse than vaginal approach for prolapse, but is related to a longer return to daily activities. ${ }^{2}$ In order to avoid this long recovery time, a minimally invasive approach for sacrocolpopexy has been used. The current literature describes objective cure rates for the apical compartment to be $97-100 \%$ after robotassisted sacrocolpopexy (RASC). ${ }^{5}$ However, these results are mostly based on short- to mid-term time frames. Solely a few studies describe outcomes more than 24 months after surgery. ${ }^{5}$ In addition, long-term postoperative results on patient reported outcomes are lacking. Female POP influences quality of life as well as day-to-day activities, emphasizing the need for long-term subjective results even more. ${ }^{6}$ The objective of this study was to evaluate whether RASC or robot-assisted supracervical hysterectomy with sacrocervicopexy (RSHS) leads to both long-term improved anatomic results as well as subjective patient reported outcomes. As the anterior compartment is victim of most recurrences, we specifically focus on this and the occurrence of postoperative micturition symptoms, as these often occur after pelvic floor repair. ${ }^{7}$

\section{Methods}

\section{Study design}

This study is part of the PARSEC database: Prospective Assessment of Robotic Sacrocolpopexy: a European Multicentric Cohort (https://clinicaltrials.gov; identifier NCT01598467). This study was carried out in accordance with the ethical standards of the Central Committee on Research Involving Human Subjects (CCMO).

\section{Patients and Evaluation}

All patients with symptomatic vaginal prolapse, who underwent RASC or RSHS in 2011 and 2012 were included. Stages of prolapse were identified with aid of the simplified Pelvic Organ Prolapse Quantification (sPOPQ). ${ }^{8}$ SPOPQ describes four vaginal landmarks 
(Ba: anterior vaginal wall; Bp: posterior vaginal wall; C: vaginal cuff/cervix; D: fornix posterior). Examples of the SPOPQ stages are shown in Figure 1. Patients were treated in our hospital with tertiary referral function for patients with pelvic organ prolapse (POP). Surgery was performed by an urogynaecologist. Patients were advised of alternative treatments available to them and informed about the risks and benefits of the procedure. Inclusion criteria were patients with symptomatic vaginal vault prolapse SPOPQ stage $\geq 2$. Exclusion criteria were a poor health status with inability to undergo general anaesthesia, age $<18$ years, $\geq 3$ laparotomic surgeries, planned pregnancy, known pelvic malignancies.

Primary outcome measure was objective anatomic cure rate, defined as SPOPQ stage 1 for all anatomic landmarks. Retreatments regarding recurrent prolapse were scored. Secondary outcomes were patient reported outcomes on quality of life and pelvic floor functions. Examination of patients and evaluation of complaints with a questionnaire were obtained preoperatively and at 1 and 5 years postoperatively. The questionnaires included questions regarding symptoms of vaginal bulge (seeing and/or sensation), micturition symptoms (Urogenital Distress Inventory; UDI-6) ${ }^{9}$, and quality of life (Pelvic Floor Impact Questionnaire; PFIQ-7) ${ }^{10}$. The UDI-6 questionnaire consists of three subdomains with each two questions, describing irritative, stress and obstructive micturition complaints. ${ }^{9}$ Question number 2 and 3 of the UDI-6 'do you experience urinary leakage related to feelings of urgency' and 'do you experience urinary leakage during physical activity, coughing or sneezing?' were described separately. The answer 'not at all' to these questions of the UDI-6 (in combination with no incontinence during Valsalva manoeuvres with a full bladder at clinical consultation), were defined as success for stress urinary incontinence (SUI) and urge urinary incontinence (UUI) respectively. Participants answer the UDI-6 using one of four options: 'not at all (0)', 'slightly (1)', moderately (2)', 'greatly (3)'. To calculate the total score, no more than two items can be missing. The mean score of the six items is multiplied by $331 / 3$, resulting in a total score that ranges from 0-100. A higher score indicates more symptom distress. The PFIQ-7 includes the Incontinence Impact Questionnaire (IIQ-7), Colorectal-Anal Impact Questionnaire (CRAIQ-7), and Pelvic Organ Prolapse Impact Questionnaire (POPIQ-7). ${ }^{10}$ Answers are scored similar to the UDI-6 (0-3; 'not at all' to 3 'greatly'). Each subscale ranges from 0-100 (mean score x $331 / 3$ ). The total score is the sum of all three subscales (0-300). A higher score indicates an increased negative impact on daily life.

Patients with no postoperative consultation available, and who also did not sent in a questionnaire were considered lost to follow-up. 
Figure 1. Example of prolapse before and after surgery and optimal surgical outcomes

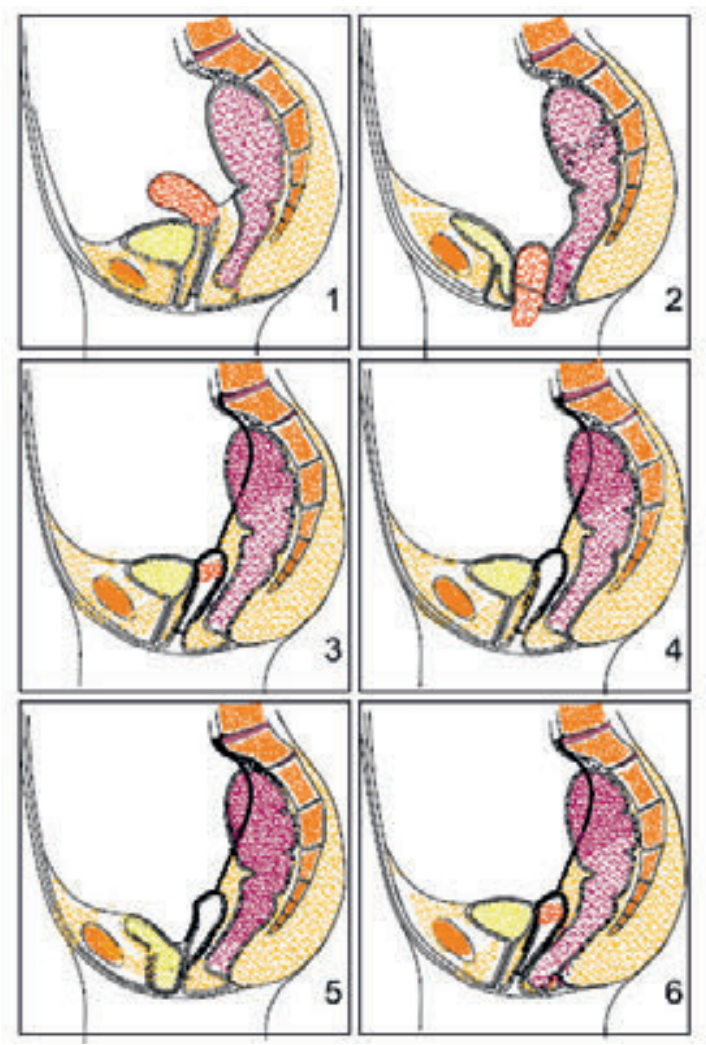

SPOPQ stage 1 describes either no prolapse or a minimal prolapse ( $>1 \mathrm{~cm}$ above the hymnal remnants). In stage 2 , the given point descends $1 \mathrm{~cm}$ above until and $1 \mathrm{~cm}$ below the hymnal remnants. Stage 3 describes a prolapse which descends more than $1 \mathrm{~cm}$ beyond the hymenal remnants, but does not represent stage 4 , which includes complete vaginal vault eversion or complete procidentia uteri. Stage 0 does not exists by definition of the SPOPQ system.

1. No prolapse.

2. Stage 3 prolapse of bladder, uterus and rectum.

3. Ideal anatomic situation after RSHS.

4. Ideal anatomic situation after RASC.

5 . Stage 3 prolapse of anterior wall after RASC.

6. Stage 2 prolapse of posterior wall after RSHS.

The black line represents the hymnal remnants.

RASC Robot-assisted sacrocolpopexy RSHS Robot-assisted supracervical hysterectomy with sacrocervicopexy 


\section{Surgical Technique}

The surgical technique used has been described previously. ${ }^{11}$ In short all procedures were performed with robotic assistance using the da Vinci Si HD (Intuitive Surgical, Inc, Sunnyvale, CA). Prolene mesh was used (Prolene, Ethicon Inc, Johnson \& Johnson, Hamburg, Germany). Proximal attachment to the sacral promontory was performed with titanium tacks (Autosuture Protack $5 \mathrm{~mm}$, Covidien, Mansfield, MA). Distally, the mesh was attached with nonabsorbable sutures (Ethibond, Ethicon Inc.) to the anterior and posterior vaginal wall and to the vaginal apex/cervix. If the uterus was present, a supracervical hysterectomy was performed. The peritoneum was closed with a $23-\mathrm{cm} \mathrm{V-}$ Loc suture (Covidien). At the end of the procedure, a vaginal examination was performed by the urogynaecological surgeon to evaluate the correction of the prolapse. Postoperatively, all of the patients were prescribed a laxative (Macrogol 3350/electrolytes, Movicolon, Norgine Limited, Mid Glamorgan, United Kingdom). Patients were advised to refrain from postoperative heavy lifting and sexual intercourse for 6 weeks postoperatively.

\section{Statistical Analysis}

Statistical analysis was performed using SPSS v. 22.0 (IBM Corp., Armonk, NY, USA). A pvalue of $<0.05$ was considered significant. Data were presented as mean \pm SD or median and range for normally and non-normally distributed continues values respectively. Number and percentages were used for nominal and categorical values. Independentsamples T-test, Mann-Whitney $U$ test, $X^{2}$ test were used to compare data for mean, median and nominal values respectively. Paired T-test, Wilcoxon signed rank test and McNemar test were used to compare scores before and after surgery as appropriate.

\section{Results}

In total, 77 patients were included (Figure 2). Patients had a mean age of $63.1 \pm 10.3$ years and BMI of $26.0 \pm 3.5$ (Table 1). One surgery was converted to an open procedure due to anaesthetic related problems. Ten patients (13\%) had a concomitant placement of a Tension-free vaginal tape (TVT) because of severe preoperative SUI. A concomitant anterior or posterior colporrhaphy was performed in 11 patients $(14.3 \%)$. In 61 patients $(79.2 \%)$ long term follow-up was available with a mean follow-up of $49.6 \pm 6.6$ months. Of these 61 patients, seven responded with a questionnaire only: the questionnaire was sent back by mail. Patients who were seen for follow-up in our outpatient clinic were 
examined by an independent (not the surgeon) researcher (FZ). During consultation, three patients declined physical examination, because these patients judged this exam unnecessary as they had no complaints.

Figure 2. Flow chart of included patients

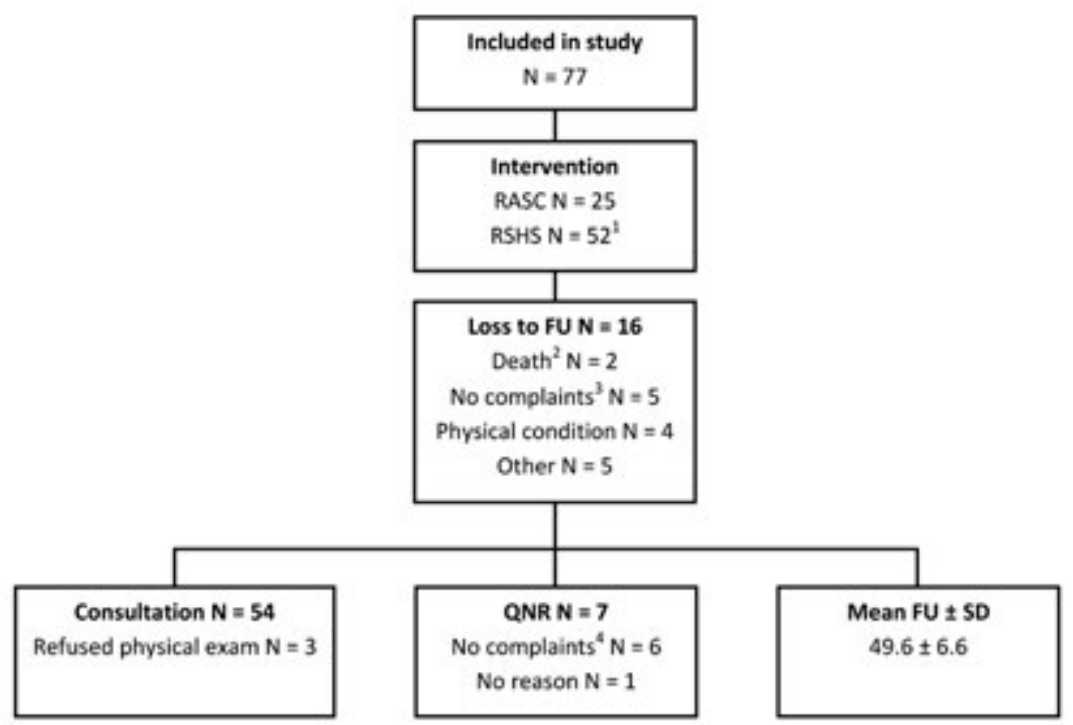

${ }^{1}$ One hysteropexy, decided during surgery ${ }^{2}$ Due to natural causes ${ }^{3}$ Patients had no complaints and therefore refused consultation ${ }^{4}$ Patients had no complaints and therefore declined consultation in the outpatient clinic, but did return a QNR

$F U$ follow up $N$ number $Q N R$ questionnaire $S D$ standard deviation

\section{Anatomical results.}

The pre- and postoperative stages of the SPOPQ are shown in Table 2 per anatomic landmark. The percentages presented describe preoperatively the 77 patients included and postoperatively the 51 patients with a postoperative physical examination available. Thirty patients $(30 / 51 ; 58.8 \%)$ were completely recurrence free at final follow-up. Patients with a vaginal prolapse present, mostly consisted of stage $2 \mathrm{sPOPQ}$ prolapse. After 50 months $96 \%$ of patients had no apical recurrence. Two stage 4 recurrent apical prolapses were seen (3.9\%) Both patients had a stage 4 apical prolapse preoperatively. Most recurrences were in the anterior compartment (Table 2). There were 16 patients $(31.4 \%)$ with a recurrent cystocele, mostly consisting of a mild stage 2 cystocele without complaints and no whish for repeat surgery $(n=10,19.6 \%)$. Two patients $(3.9 \%)$ received a re-intervention due to prolapse: 1 anterior colporrhaphy and 1 ring pessary. 
Table 1. Baseline demographics $\mathrm{N}=77$

\begin{tabular}{|c|c|c|c|}
\hline Age & $63.1 \pm 10.3$ & \multicolumn{2}{|l|}{ Sexually active } \\
\hline BMI & $26.0 \pm 3.5$ & No & $27(35.1)$ \\
\hline \multirow{2}{*}{ Parity } & \multirow{2}{*}{$3(0-11)$} & Yes & $40(51.9)$ \\
\hline & & Not reported & $10(13.0)$ \\
\hline Postmenopausal & $63(81.8)$ & Smoking (active) & $14(18.2)^{\mathrm{b}}$ \\
\hline Prev hysterectomy & $25(32.5)$ & Vaginal estrogens & $2(2.6)$ \\
\hline Previous POP/inc surgery & $29(37.7)$ & \multicolumn{2}{|l|}{ Preoperative sPoPQ } \\
\hline Intra-abd. surgerya & $33(42.9)$ & A & $2.3 \pm 1.0$ \\
\hline \multirow{2}{*}{ Sphincter rupture labour } & \multirow{2}{*}{$2(2.6)$} & $\mathrm{B}$ & $2.2 \pm 1.0$ \\
\hline & & $\mathrm{C}$ & $2.2 \pm 1.0$ \\
\hline Episiotomy labour & $35(45.5)$ & $\mathrm{D}^{\mathrm{c}}$ & $1.4 \pm 0.9$ \\
\hline \multicolumn{2}{|l|}{ ASA score } & Pre-operative SUI & $34(44.2)$ \\
\hline 1 & $25(32.5)$ & \multirow[t]{3}{*}{ Pre-operative UUI } & \multirow[t]{3}{*}{$32(41.6)$} \\
\hline 2 & $49(63.6)$ & & \\
\hline 3 & $3(3.9)$ & & \\
\hline \multicolumn{4}{|c|}{ Numbers presented as mean \pm SD, median (range) or number (\%). } \\
\hline \multicolumn{4}{|c|}{$\begin{array}{l}A S A \text { American Society of Anesthesiologist } B M I \text { body-mass index; inc: incontinence } N \text { number } P O P \\
\text { pelvic organ prolapse post posterior } S P O P Q \text { simplified pelvic organ prolapse quantification } S U I \text { stress } \\
\text { urinary incontinence } U U I \text { stress urinary incontinence }\end{array}$} \\
\hline \multicolumn{4}{|c|}{ 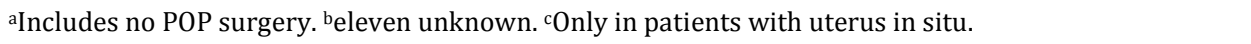 } \\
\hline
\end{tabular}


Table 2. Pre- and postoperative anatomic results

\section{Preoperative sPOPQ}

Anterior

compartment

\section{Posterior}

compartment

\section{Apical}

compartment

\section{Posterior}

fornix $^{a, b}$
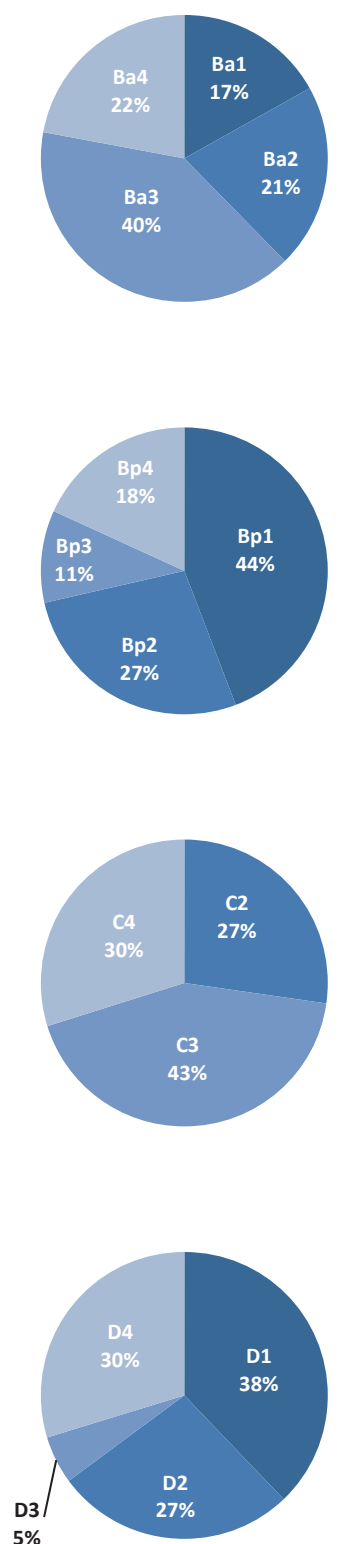

\section{Postoperative SPOPQ}
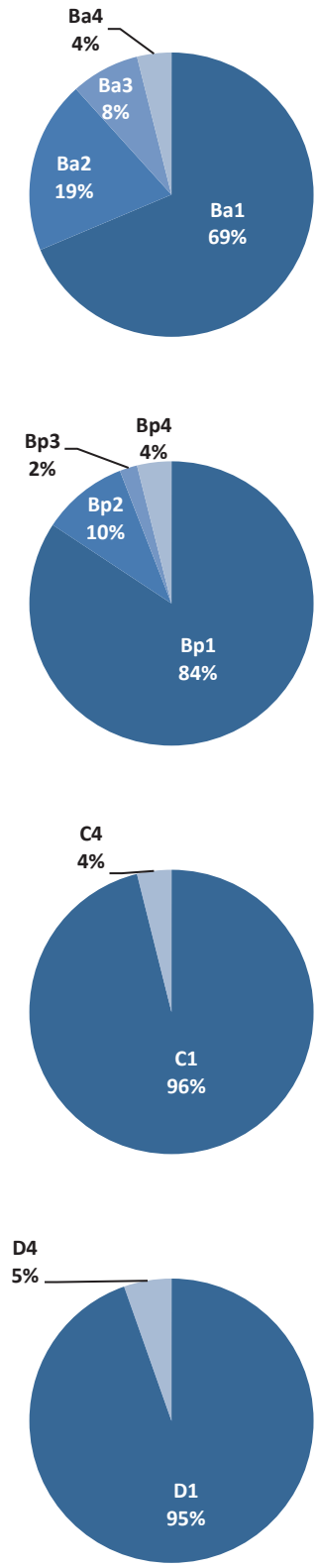

SPOPQ simplified pelvic organ prolapse quantification (stage).

aOnly when cervix is present: 25/77 patients had no cervix preoperatively, 14/51 postoperatively. bIn 15 patients 'landmark D' was not described preoperatively. 
The median SPOPQ stages of all four anatomic landmarks improved significantly from pre- to postoperative visit [median preoperative and postoperative stages: SPOPQ Ba: 3.0 to $1.0(p<0.0005)$ sPOPQ Bp: 2.0 to $1.0(p<0.0005)$; sPOPQ C: 3.0 to $1.0(p<0.0005)$; sPOPQ D: 2.0 to $1.0(p<0.010)]$.

\section{Patient reported outcome measures}

Symptoms of bulge improved from $95 \%$ to $10 \%(p<0.0005$; Table 3). Quality of life scores also improved significantly, mainly due to improved incontinence and POP impact scores. Colorectal quality of life scores were low both pre- and postoperatively, and did not change. The total UDI-6 scores after five years improved significantly ( $p=0.048)$. Exploring the three subdomains, improvement of urinary symptoms was mostly caused by enhancement of obstructive micturition. Twenty percent of patients showed to suffer from, mostly mild, de novo SUI at follow-up (slightly 16\%; moderately $1.6 \%$; greatly $1.6 \%$ ). In $14 \%$ of all patients, SUI symptoms resolved completely postoperatively. Three patients (4.9\%) needed a TVT postoperatively. Of the ten patients receiving a TVT during surgery, two patients had persistent complaints of moderate SUI. De novo UUI occurred in 10 patients (16\%), with half of patients having mild complaints postoperatively. Spontaneous relieve of UUI symptoms occurred in $6 \%$ of patients. No difference in complaints of urinary frequency or nocturia was observed.

Table 3. Patient reported outcome measures

\begin{tabular}{lccc}
\hline & $\begin{array}{c}\text { Preoperative } \\
\mathbf{N = 7 7}\end{array}$ & $\begin{array}{c}\text { Postoperative } \\
\mathbf{N = 6 1}\end{array}$ & $\boldsymbol{p}$-value \\
\hline Bulge symptoms & $73(94.8)$ & $6 / 61(9.8)$ & $<0.0005$ \\
PFIQ-7 total (0-300) & $60.0(0-185.7)$ & $0(0-300)$ & 0.008 \\
UIQ-7 (0-100) & $16.7(0-90.5)$ & $0(0-100)$ & $0.016^{*}$ \\
CRAIQ-7 (0-100) & $0(0-57.1)$ & $0(0-100)$ & 0.051 \\
POPIQ-7 (0-100) & $31.0(0-95.2)$ & $0(0-100)$ & $0.005^{*}$ \\
UDI-6 total (0-100) & $26.7(0-93.3)$ & $22.2(0-72.2)$ & $0.048^{*}$ \\
Irritative (0-100) & $33.3(0-100)$ & $33.3(0-100)$ & 0.450 \\
Stress (0-100) & $33.3(0-100)$ & $16.7(0-100)$ & 0.574 \\
Obstructive (0-100) & $33.3(0-100)$ & $0.0(0-100)$ & $0.008^{*}$ \\
Urine-incontinence & & & \\
Stress urine-incontinence & & & 1.000 \\
No & $55 \%$ & $48 \%$ & \\
Yes & $45 \%$ & $52 \%$ & \\
\hline
\end{tabular}


Table 3. Continued

\begin{tabular}{|c|c|c|c|}
\hline & $\begin{array}{l}\text { Preoperative } \\
\qquad N=77\end{array}$ & $\begin{array}{l}\text { Postoperative } \\
\qquad \mathrm{N}=61\end{array}$ & $p$-value \\
\hline Urge urine-incontinence & & & 0.454 \\
\hline Yes & $59 \%$ & $53 \%$ & \\
\hline No & $41 \%$ & $47 \%$ & \\
\hline \multicolumn{4}{|l|}{ Urinary symptoms } \\
\hline Urgency & $45(63.4)$ & $24(31.2)$ & 0.026 \\
\hline Frequency ( $\geq 8$ times/day) & $32(41.6)$ & $21(27.3)$ & 0.167 \\
\hline Nycturia ( $\geq 2 /$ night $)$ & $25(36.2)$ & $27(35.1)$ & 0.454 \\
\hline Recurrent UTI ( $\geq 2$ /year) & $11(14.3)$ & $6(9.8)$ & 0.289 \\
\hline \multicolumn{4}{|c|}{$\begin{array}{l}\text { Data presented as number (percentage), median (range). } \\
\text { CRAIQ-7 Colorectal-Anal Impact Questionnaire NA not applicable PFIQ-7 Pelvic Floor Impact } \\
\text { Questionnaire POPIQ-7 Pelvic Organ Prolapse Impact Questionnaire POPQ pelvic organ prolapse } \\
\text { quantification SUI stress urinary incontinence UDI-6 Urinary Distress Inventory UIQ-7 Urinary Impact } \\
\text { Questionnaire UUI urge urinary incontinence }\end{array}$} \\
\hline
\end{tabular}

\section{Discussion}

\section{Main findings}

Ninety-six percent of patients had no apical recurrence after 50 months of follow-up. Thirty patients (58.8\%) showed no vaginal prolapse in any compartment. Most recurrences were sPOPQ stage 2 cystoceles with no patient reported symptoms of vaginal bulge. The clinical relevance of these asymptomatic recurrences remains unclear. This finding is consistent with the study of Slieker et al. ${ }^{12}$ An epidemiologic study in which a high rate of stage 2 prolapse was found in women who reported not to have any complaints.

For the patients, the most important findings pertained to improvement in functional wellbeing. Symptoms of bulge and quality of life improved substantially. Patients should be counselled that de occurrence of 'de novo' SUI is not infrequent, although it is usually mild and only in a low percentage additional incontinence surgery is necessary (5\%).

\section{Strengths and limitations}

A strong point of this study is the long follow-up period. Results of improvement after RASC have been shown on the short and mid-term, however few studies report longterm anatomic and subjective results. Secondly, this study was set up as a prospective 
cohort study. Loss to follow-up was known in most cases. Some of these patients were willing to return the validated questionnaire via mail, improving our ability to measure long-term results. Patients were treated in a single tertiary referral centre for prolapse. This limited generalizability. Secondly, the limited sample size meant low power to perform logistic regression that determines risk factors for recurrence.

\section{Interpretation}

There are only a few studies reporting long-term results after sacrocolpopexy. Linder et al. performed a retrospective study on 70 patients undergoing RASC (median follow-up 72 months). ${ }^{13}$ The study identified four patients (5.7\%) who needed repeat surgery for recurrent prolapse, including one for apical recurrence. These numbers are in line with our $96 \%$ recurrence free interval. In a retrospective cohort study evaluating 95 patients receiving RASC, persistent POP was observed in $4.2 \%$ after 34 months. ${ }^{14}$ Follow-up was scheduled at 1 month and 12 months, and a phone interview in case patients were not recently assessed. The percentage of recurrences is lower than our objective results, but this number could be underestimated based on different study design. A recent prospective cohort study included 331 patients who underwent laparoscopic sacrocolpopexy (LSC), of which 270 patients were seen or interviewed for follow-up ( $n=185$ physically exam, interview; n=95 interview). After 85.5 months, 83\% reported improvement based on the Patient Global Impression of Change score, 7\% reported clear deterioration. Apical recurrence was reported in $9 \%$ of patients and anterior and posterior prolapse recurrences were more common (22\% and $29 \%$, respectively). The reintervention rate for prolapse was 3.3\%, comparable to our results. ${ }^{15}$

One study, which described both RASC and RSHS ( $\mathrm{N}=33)$, changed absorbable sutures for non-absorbable sutures after one apical recurrence in which the mesh had detached from the underlying tissue. ${ }^{16}$ In our cohort only non-resorbable sutures were used. One randomized trial compared LSC $(\mathrm{N}=61)$ with ASC $(\mathrm{N}=60)$ [follow-up 41.7 months]. ${ }^{17}$ Cure, defined as apical prolapse stage $\leq 1$ (based on the Pelvic Organ Prolapse Quantification ${ }^{18}$ ), point $C / D \leq-5$, and $\geq 7 \mathrm{~cm}$ total vaginal length, was $100 \%$ for both approaches. This study performed a secondary analysis in which any kind of prolapse (including asymptomatic) in any compartment was evaluated. Patients who underwent LSC, showed significantly earlier recurrence, mostly within 12 months of surgery. Anterior POP recurrences were significantly higher after LSC. 


\section{Quality of life}

Studies describing quality of life after RASC based on validated questionnaires are scarce. One large cohort study $(\mathrm{N}=150)$ with one-year follow-up evaluations, showed improved PFIQ-7 scores from 59 to 6.5 (p<.0001). ${ }^{19}$ These results are in line with our scores. Another smaller study $(\mathrm{N}=50)$, reported long-term QoL after robot-assisted hysteropexy based on a visual analogue scale. QoL scores improved significantly. ${ }^{20}$

\section{Anterior compartment}

In general, the most common type of prolapse is prolapse of the anterior compartment. ${ }^{21}$ Treatment of this compartment remains challenging and many different methods have been described.4,21 Mild prolapses on examination are seen often and they are frequently asymptomatic. A long-term study (four years) reported 55\% recurrent cystoceles (stage $\geq 2$ ) after anterior colporrhaphy. ${ }^{22}$ In this study, after sacrocolpopexy, we found a lower recurrent anterior wall rate of $31 \%$. Loss of apical support often occurs in women with anterior wall prolapse that extends beyond the hymen. ${ }^{23}$ Chen et al. looked at the interaction between the pubovisceral muscle and apical support. ${ }^{24}$ Results suggested that prolapse can develop as a result of impairment of the pubovisceral muscle and apical vaginal suspension. The larger the pubovisceral impairment, the larger the anterior wall prolapse becomes. Suspension of the apical compartment in patients with significant anterior wall descend is important but does not repair the pubovisceral muscle. This could possibly explain the occurrence of anterior wall prolapse after RASC/RSHS. After combined apical and cystocele repair procedures, a significantly lower prolapse reoperation rate was seen than in women with an isolated anterior wall repair ( 11.6 vs $20.2 \%, p<0.01) .{ }^{23}$ In this study, preoperatively $83 \%$ of women had an anterior wall prolapse ( $22 \%$ stage 4 ), compared to $31 \%$ postoperatively ( $4 \%$ stage 4 ). As opening the vaginal fascia during anterior colporrhaphy could theoretically lead to more mesh exposure, further research should first be performed, before combining RASC with concomitant anterior colporrhaphy in women with combined anterior and middle compartment prolapse stage 3-4.

The degree of anterior dissection and tensioning of the mesh is an important step in a sacrocolpopexy procedure, but these steps are mostly based on experience. ${ }^{25}$ Recent studies suggest that more caudal anterior dissection could lead to less recurrences. On the other hand, placement of the mesh too caudal can possibly lead to new functional symptoms such as urine-incontinence. ${ }^{7,25}$ Future research should be focused on this subject. 


\section{Micturition symptoms}

Preoperative bladder over activity may resolve in as much as $40 \%$ of patients undergoing POP surgery. On the other hand, de novo bladder over activity occurs in $12 \% .7,26$ Costantini et al. described de novo urgency after RASC in $10.5 \%$ of patients, but most complaints resolved in the first postoperative weeks or after treatment with anticholinergics. ${ }^{17} \mathrm{We}$ found complaints of urgency to diminish from $63 \%$ to $31 \%$ and de novo UUI occurred in 16\%. UDI-6 irritative impact scores remained similar.

De novo SUI after POP surgery has a wide range of occurrence, ranging from 16 to $51 \% .{ }^{27} \mathrm{~A}$ systematic review on urine incontinence after prolapse surgery found a lower number needed to treat (NNT) for women with SUI or continent women with occult SUI after prolapse surgery combined with an anti-incontinence procedure than the NNT to prevent one women developing de novo SUI in all continent women. ${ }^{28}$ Other reviews highlight this finding, but heterogeneity among studies is high. Wide ranges of additional anti-incontinence surgery after prolapse repair were reported: $28 \%$ in women with SUI, $4-16 \%$ in asymptomatic women without occult SUI, and $1.5-24 \%$ in women with occult SUI. ${ }^{28}$ Data are inconsistent on whether a prophylactic colposuspension should be performed in continent women undergoing sacrocolpopexy. ${ }^{7}$ The Colpopexy and Urinary Reduction Efforts trial reported de novo SUI to be $44.1 \%$ after abdominal sacrocolpopexy without a Burch procedure. ${ }^{27}$ A retrospective study evaluated de novo SUI in women with no preoperative occult SUI. The authors found no difference in de novo SUI rates between apical and non-apical POP procedures $(9.7 \%$ vs $10.5 \%$ respectively). ${ }^{27}$ They did identify sacrocolpopexy and patients with preoperative SUI as risk factors for de novo SUI. Both vaginal as abdominal procedures were included in this study. As we found de novo SUI which needed to be treated surgically in 5\%, patients should be counselled preoperatively of this possibility.

\section{Conclusions}

RASC and RSHS show sustainable results in the treatment of prolapse. After a follow-up of 50 months, $96 \%$ of patients showed no apical recurrence. Roughly one in nine patients can expect a symptomatic anterior wall recurrence. Patient reported outcome measures, such as symptoms of bulge and quality of life, improved substantially. Patients should preoperatively be counselled about the risk of 'de novo' SUI. 


\section{References}

1. Smith FJ, Holman CDJ, Moorin RE, Tsokos N. Lifetime risk of undergoing surgery for pelvic organ prolapse. Obstet Gynecol. 2010;116:1096-1100.

2. Maher C, Feiner B, Baessler K, Glazener CMA. Surgical management of pelvic organ prolapse in women. Cochrane Database of Syst Rev. 2013;(4):CD004014:1-138.

3. Clark AL, Gregory T, Smith VJ, Edwards R. Epidemiologic evaluation of reoperation for surgically treated pelvic organ prolapse and urinary incontinence. Am J Obstet Gynecol. 2003;189:1261-7

4. Lensen EJM, Withagen MIJ, Kluivers KB, Milani AL, Vierhout ME. Surgical treatment of pelvic organ prolapse: a historical review with emphasis on the anterior compartment. Int Urogynecol J. 2013;24:1593-1602.

5. Serati M, Bogani G, Sorice P, Braga A, Torella M, Salvatore S, et al. Robot-assisted sacrocolpopexy for pelvic organ prolapse: a systematic review and meta-analysis of comparative studies. Eur Urol. 2014;66:303-318.

6. 't Hoen LA, Utomo E, Steensma AB, Blok BFM, Korfage IJ. The pelvic organ prolapse/urinary incontinence sexual questionnaire (PISQ-12): validation of the Dutch version. Int Urogynecol J. 2015;26:1293-303.

7. Baessler K, Maher C. Pelvic organ prolapse surgery and bladder function. Int Urogynecol J. 2013;24:1843-1852.

8. Swift S, Morris S, McKinnie V, Freeman R, Petri E, Scotti RJ, et al. Validation of a simplified technique for using the POPQ pelvic organ prolapse classification system. Int Urogynecol J. 2006;17:615-620.

9. Utomo E, Korfage IJ, Wildhagen MF, Steensma AB, Bangma CH, Blok BF. Validation of the Urogenital Distress Inventory (UDI-6) and Incontinence Impact Questionnaire (IIQ-7) in a Dutch population. Neurourol Urodyn. 2015;34:24-31.

10. Utomo E, Blok BF, Steensma AB, Korfage IJ. Validation of the Pelvic Floor Distress Inventory (PFDI20) and Pelvic Floor Impact Questionnaire (PFIQ-7) in a Dutch population. Int Urogynecol J. 2014;25:531-544.

11. van Zanten F, Schraffordt Koops SE, O'Sullivan OE, Lenters E, Broeders IAMJ, O'Reilly BA. Robotassisted surgery for the management of apical prolapse: a bi-centre prospective cohort study. BJOG. 2019;126:1065-1073.

12. Slieker-ten Hove MCPh, Pool-Goudzwaard AL, Eijkemans MJC, Steegers-Theunissen RPM, Burger CW, Vierhout ME. Prediction model and prognostic index to estimate clinically relevant pelvic organ prolapse in a general female population. Int Urogynecol J. 2009;20:1013-1021.

13. Linder BJ, Chow GK, Elliott DS. Long-term quality of life outcomes and retreatment rates after robotic sacrocolpopexy. Int J Urol. 2015;22:1155-1158.

14. Ploumidis A, Spinoit A-F, De Naeyer G, Schatteman P, Gan M, Ficarra V, et al. Robot-assisted sacrocolpopexy for pelvic organ prolapse: surgical technique and outcomes at a single high-volume institution. Eur Urol. 2014;65:138-145.

15. Pacquée S, Nawapun K, Claerhout F, Werbrouck E, Veldman J, D’hoore A, et al. Long-term assessment of a prospective cohort of patients undergoing laparoscopic sacrocolpopexy. Obstet Gynecol. 2019;134:323-32. 
16. Benson AD, Kramer B a, Wayment RO, Schwartz BF. Supracervical robotic-assisted laparoscopic sacrocolpopexy for pelvic organ prolapse. JSLS. 2010;14:525-530.

17. Costantini E, Mearini L, Lazzeri M, Bini V, Nunzi E, di Biase M, et al. Laparoscopic versus abdominal sacrocolpopexy: a randomized, controlled trial. J Urology. 2016;196:159-165.

18. Bump RC, Mattiasson A, Bo K, Brubaker LP, DeLancey JO, Klarskov P, et al. The standardization of terminology of female pelvic organ prolapse and pelvic floor dysfunction. Am J Obstet Gynecol. 1996;175:10-7.

19. Culligan PJ, Gurshumov E, Lewis C, Priestly JL, Komar J, Shah N, et al. Subjective and objective results 1 year after robotic sacrocolpopexy using a lightweight Y-mesh. Int Urogynecol J. 2014;25:731-5.

20. Mourik SL, Martens JE, Aktas M. Uterine preservation in pelvic organ prolapse using robot assisted laparoscopic sacrohysteropexy: quality of life and technique. Eur J Obstet Gynecol Reprod Biol. 2012;165:122-7.

21. Durnea CM, Pergialiotis V, Duffy JMN, Bergstrom L, Elfituri A, Doumouchtsis SK, CHORUS, an International Collaboration for Harmonising Outcomes, Research and Standards in Urogynaecology and Women's Health. A systematic review of outcome and outcome-measure reporting in randomised trials evaluating surgical interventions for anterior-compartment vaginal prolapse: a call to action to develop a core outcome set. Int Urogynecol J. 2018;29:1727-1745.

22. Wong V. Shek KL, Goh J, Krause H, Martin A, Dietz HP. Cystocele recurrence after anterior colporrhaphy with and without mesh use. Eur J Obstet Gynec Reprod Biol. 2014;172:131-135.

23. Alas AN, Anger JT. Management of apical pelvic organ prolapse. Curr Urol Rep. 2015;16:33.

24. Chen L, Ashton-Miller JA, Hsu Y, DeLancey JOL. Interaction between apical supports and levator ani in anterior vaginal support: theoretical analysis. Obstet Gynecol. 2006;108:324-332.

25. Habib N, Centini G, Pizzoferrato A-C, Bui C, Istvan A, Bader G. Laparoscopic promontofixation: Where to stop the anterior dissection? Medical Hypotheses. 2019;124:60-63.

26. Hamamsy DEJ, Fayyad AM. New onset stress urinary incontinence following laparoscopic sacrocolpopexy and its relation to anatomical outcomes. Int Urogynecol J. 2015;26:1041-1045.

27. Alas AN, Chinthakanan O, Espaillat L, Plowright L, Davila GW, Aguilar VC. De novo stress urinary incontinence after pelvic organ prolapse surgery in women without occult incontinence. Int Urogynecol J. 2016;28:583-590.

28. van der Ploeg JM, van der Steen A, Oude Rengerink K, van der Vaart CH, Roovers JP. Prolapse surgery with or wihtout stress incontinence surgery for pelvic organ prolapse: a systematic review and meta-analysis of randomised trials. BJOG. 2014;121:537-547. 




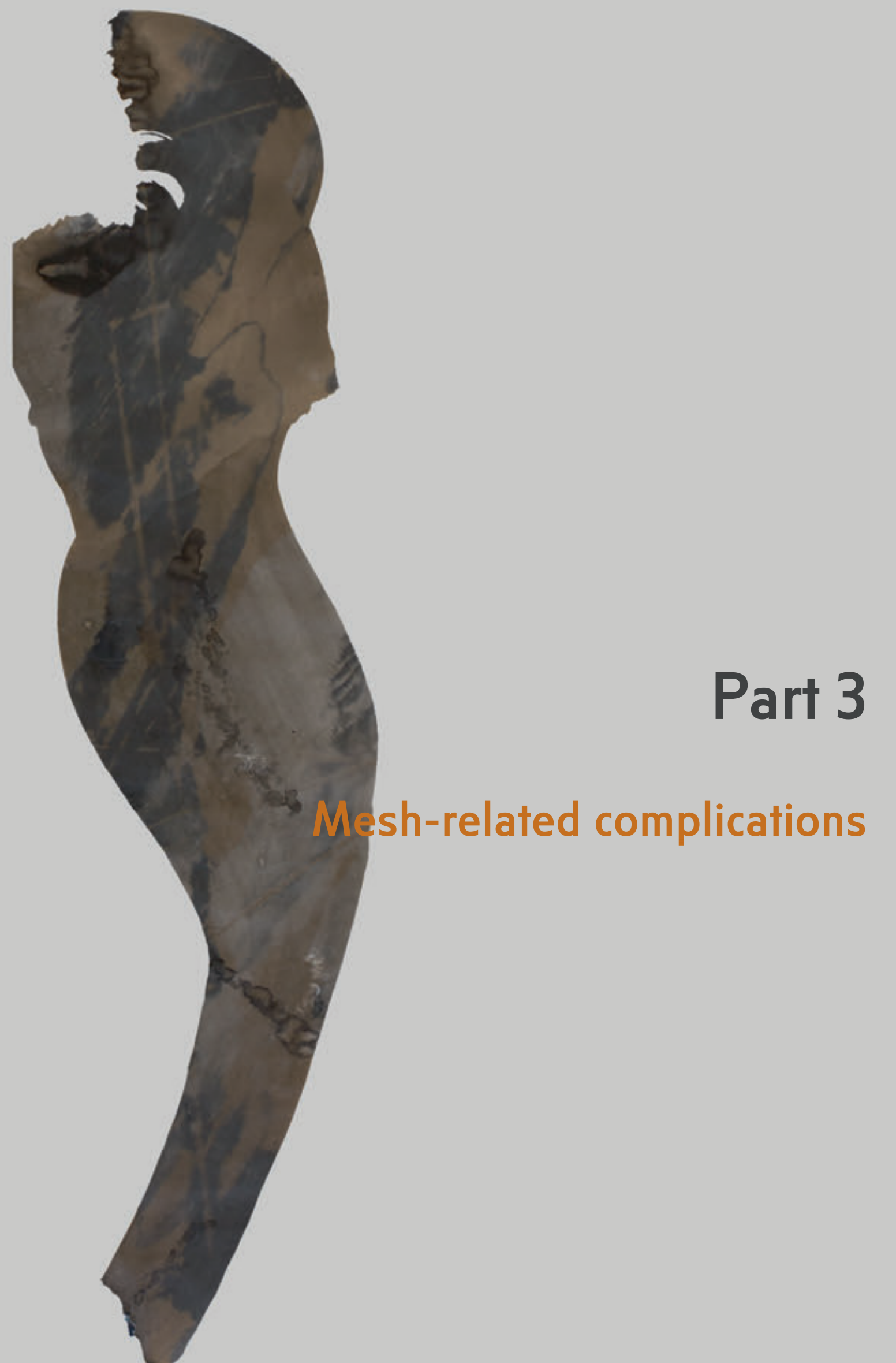





\section{Chapter 7}

Mesh exposure after robot-assisted laparoscopic pelvic floor surgery: a prospective cohort study

Femke van Zanten, Jan J. van lersel, Francis E. Hartog Karin I.M. Aalders, Egbert Lenters, Ivo A.M.J. Broeders, Steven E. Schraffordt Koops 


\section{Abstract}

Study objective: To prospectively evaluate the mesh exposure rate after robot-assisted laparoscopic pelvic floor surgery for the treatment of female pelvic organ prolapse (POP) in a large cohort.

Design: Prospective observational cohort study (Canadian task force classification II-2).

Setting: Two large teaching hospitals with a tertiary referral function for pelvic floor disorders.

Patients: Patients with symptomatic POP and simplified POP quantification (sPOPQ) stage $\geq 2$. Patients with a history of mesh repair or concomitant insertion of a tensionfree vaginal tape were excluded.

Interventions: Robot-assisted laparoscopic sacrocolpopexy or robot-assisted laparoscopic supracervical hysterectomy with a sacrocervicopexy.

Measurements and Main Results: A vaginal examination with the aid of a transparent speculum was performed to look for mesh-related complications. Mesh exposures were described following the International Urogynecological Association/International Continence Society classification system. One hundred and ninety-two patients were included, of whom 166 patients (86.5\%) were seen for follow-up examination. The median duration of follow-up was 15.7 months (range, 8.2-44.4 months). Two vaginal mesh exposures $(1.2 \%)$ were detected, both of wich were treated in the outpatient clinic. One patient without any complaints had a suture exposure, which was removed in the outpatient clinic.

Conclusion: The safety of the use of mesh in pelvic floor surgery is a matter of debate owing to the occurrence of mesh-related complications. Based on the current literature, mesh-related complications seem to be lower in transabdominal mesh surgery than in transvaginal mesh surgery. In this study, a low mesh exposure rate was observed in robot-assisted abdominal pelvic floor surgery for POP. 


\section{Introduction}

In 2008 and 2011 the US Food and Drug Administration (FDA) published a safety communication on complications related to the use of synthetic meshes. ${ }^{1}$ The FDA based this warning on a systematic review showing a high incidence of mesh-related complications following transvaginal pelvic organ prolapse (POP) repair (incidence 10.3\%; range, 0-29.7\%;, $\mathrm{n}=11,785)$. $^{2}$ Lower rates of mesh complications are seen after abdominal surgery using mesh with a median mesh exposure rate of $4 \%$ within 23 months of surgery. ${ }^{1}$ Owing to the vigorous debate on the consequences of vaginal mesh use and worldwide litigation, patients and doctors are becoming more reserved in the overall use of mesh including in abdominal prolapse surgery. This may lead to suboptimal treatment of patients suffering from POP, resulting in a lower quality of life. Systematic reviews published after the FDA warning have reported a wide range of mesh exposure rates, including mean rates of $2 \%$ in robot-assisted laparoscopic sacrocolpopexy (RASC; range $0-8 \%$ ) and $3 \%$ in laparoscopic sacrocolpopexy (LSC; range $0-9 \%), 3,4$ However, these findings are mostly based on retrospective and/or small cohorts and may underestimate true mesh exposure rates. Given the increasing use of synthetic meshes due to a rising prevalence of female POP, 2,5 determining accurate mesh exposure rates is important.

The use of robotics in place of straight stick conventional laparoscopy, has been gaining popularity in pelvic floor repair because it may make complex minimally invasive procedures more facile. ${ }^{6}$ The aim of this study was to determine the mesh exposure rate in a large cohort of patients undergoing robot-assisted laparoscopic prolapse surgery.

\section{Materials and Methods}

This prospective cohort study was performed in two large teaching hospitals with a tertiary referral function for patients with POP. Our series is part of the PARSEC database (Prospective Assessment of Robotic Sacrocolpopexy: a European Multicentric Cohort). All patients who underwent RASC or robot-assisted laparoscopic supracervical hysterectomy with a sacrocervicopexy (RSHS) at the Meander Medical Center (May 2011- December 2015) and Rijnstate Hospital (September 2011 to June 2013) were consecutively included. The simplified Pelvic Organ Prolapse Quantification (sPOPQ) was used to determine the stage of prolapse. ${ }^{7} \mathrm{sPOPQ}$ is a validated short form of the standard $\mathrm{POPQ}$, describing only four vaginal landmarks using four grades, making it 
more clinically accessible..$^{8}$ Inclusion criteria were symptomatic POP and SPOPQ stage $\geq$ 2 (i.e., descending from the given landmark of the sPOPQ at least $1 \mathrm{~cm}$ above the hymnal remnants or lower). Exclusion criteria were age $<18$ years, inability to undergo general anesthesia, and history of 3 or more previous laparotomies. Patients with a history of previous mesh procedures or with concomitant mesh procedures were excluded as well. The primary study outcome was mesh exposure.

All patients were counseled about alternative treatments and informed of the risks and benefits of the procedure. Patients who did not undergo a postoperative vaginal examination were considered lost to follow-up. Mesh exposure was defined as any epithelial defect with visualization of the mesh through the vaginal or adjacent tissues. Protruding permanent sutures were scored separately. All exposures were described according to the International Urogynecological Association/International Continence Society classification system. ${ }^{9}$ In this classification system, the term exposure is defined as 'a condition of displaying, revealing, exhibiting, or making accessible (e.g., vaginal mesh visualized through separated vaginal epithelium)'. Patients underwent a routine follow-up examination at 12 months after surgery or when presenting with complaints. Follow-up examinations were performed by trained research fellows. Patients who did not attend the routine 1-year follow-up, were invited a second time for postoperative evaluation in 2016.

All patients underwent a vaginal examination with the aid of a transparent speculum. When mesh-related morbidity was found or suspected a second examination was performed by the urogynecologist to confirm the diagnosis. Patients completed a questionnaire before and after surgery to answer questions to elicit information regarding their sensation of prolapse. The questionnaire also included questions regarding urinary symptoms, defecation, and sexual function, and quality of life. Patients who not wish to attend the 1-year consultation were invited to return the postoperative questionnaire by mail and asked about mesh-related complaints over the telephone.

The surgical technique was similar to the technique described by Clifton et al (2016). ${ }^{10}$ The patient was placed under general anesthesia in a dorsal lithotomy position and given prophylactic intravenous antibiotics $(1000 \mathrm{mg}$. cefazolin and $500 \mathrm{mg}$. metronidazole). All surgeries were performed with the assistance of the da Vinci Robot (Intuitive Surgical, Sunnyvale, CA). A peritoneal incision was made over the sacral promontory and extended distal to an inverted J-form and an anterior and posterior 
dissection of the vaginal wall was performed. If the uterus was present a supracervical hysterectomy was performed as the first step. Suspension was performed with polypropylene (type 1, macroporous polypropylene, weight 80-85 g/m2; Prolene; Ethicon, Hamburg, Germany). A $22 \mathrm{~cm}$ long, 30-mm-diameter vaginal probe (Meekers Medical, Utrecht, The Netherlands) was used to spread the mesh. Two meshes were sutured to the anterior and posterior vaginal walls and configured into a ' $\mathrm{Y}$ ' shape intracorporeally. The mesh was distally attached using non-absorbable sutures (Ethibond; Ethicon) and anchored proximally to the sacral promontory using titanium tacks (Covidien Autosuture Protack $5 \mathrm{~mm}$, Medtronic, Minneapolis, MN0. The peritoneum was closed using a $23 \mathrm{~cm}$ Covidien V-Loc suture (Medtronic). Concomitant procedures were performed when clinically indicated.

The PARSEC database is listed on ClinicalTrials.gov (identifier NCT01598467; registration, May 2012). The National Central Committee on Research Involving Human Subject ruled this study exempt, because it encompasses standard survey and interview research as required by Dutch law. Data were processed anonymously (FvZ/JvI). Statistical analysis was performed using SPSS version 22.0 (IBM, Armonk, NY). Normally distributed values are presented as mean \pm standard deviation; non-normally distributed values, as median and range. The independent-samples $t$ test, Mann-Whitney $U$ Test, $X^{2}$ test, and Fisher's exact test were used to compare continuous and nominal data as appropriate.

\section{Results}

A total of 218 patients underwent surgery during the study period. Twenty-six patients $(11.9 \%)$ were excluded due to a history of pelvic floor mesh implants. Sixteen patients were lost to follow-up for various reasons (Figure 1). Another 10 patients preferred to respond solely by mail with the postoperative questionnaire. A total of 166 patients $(86.5 \%)$ were included in our analyses (Figure 1). Sixty-six patients (39.8\%) underwent RASC, and 100 patients (60.2\%) RSHS. Of the 66 patients undergoing RASC, 65 had a history of total hysterectomy and 1 had a history of supracervical hysterectomy. The baseline demographic data and surgical details of all patients by procedure are presented in Table 1. Compared with the patients who underwent RASC, those who underwent RSHS were younger, had a lower body mass index, and were less likely to be postmenopausal. This group underwent fewer previous POP/incontinence surgeries and had less severe prolapse to the posterior compartment. Among all patients, 3 patients 
(1.8\%) used vaginal estrogens preoperatively. Postoperative 12 patients $(7.2 \%)$ were prescribed or continued vaginal estrogens. The median duration of follow-up was 15.7 months [range, 8.2-44.4 months] and was comparable for the 2 surgical techniques [RASC, 16.1 months (range, 8.9-42.9 months); RSHS 15.6 months (range, 8.2-44.4 months); $p=0.865]$.

Figure 1. Flow-chart of included patients

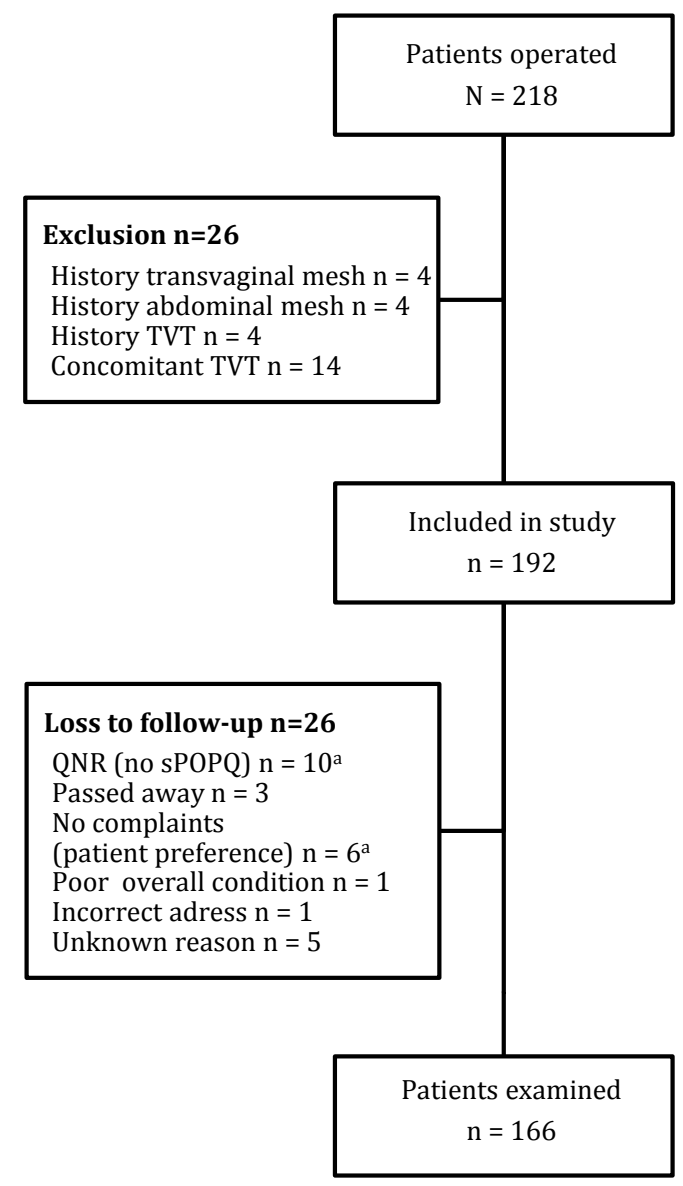

$N$ number $S P O P Q$ simplified pelvic organ prolapse quantification $T V T$ tension-free vaginal tape $Q N R$ questionnaire

aPatients either reported to have no specific mesh-related complaints in their questionnaire or by telephone. 
Table 1. Patient demographics and surgical details

\begin{tabular}{|c|c|c|c|c|}
\hline & $\begin{array}{l}\text { All patients } \\
\mathrm{N}=166\end{array}$ & $\begin{array}{l}\text { RASC } \\
n=66\end{array}$ & $\begin{array}{l}\text { RSHS } \\
n=100\end{array}$ & $P$ value ${ }^{a}$ \\
\hline Age, yr & $61.3 \pm 10.4$ & $64.8 \pm 8.4$ & $59.0 \pm 11.0$ & $<0.0005$ \\
\hline BMI $\left(\mathrm{kg} / \mathrm{m}^{2}\right)$ & $25.1(17.9-44.1)$ & $25.8(19.8-38.3)$ & $24.8(17.9-44.1)$ & 0.022 \\
\hline Parity & $3.0(0-7)$ & $2.0(0-7)$ & $3.0(1-6)$ & 0.764 \\
\hline Postmenopausal & $140(84.3)$ & $64(97.0)$ & $76(76.0)$ & $<0.0005$ \\
\hline ASA score & $2.0(1-3)$ & $2.0(1-2)$ & $2.0(1-3)$ & 0.108 \\
\hline Diabetes Mellitus & $14(8.4)$ & $6(9.1)$ & $8(8.0)$ & 0.804 \\
\hline $\begin{array}{l}\text { Pre-operative vaginal } \\
\text { estrogen }\end{array}$ & $3(1.8)$ & $2(3.0)$ & $1(1.0)$ & 0.564 \\
\hline \multicolumn{5}{|l|}{ Smoking (current) } \\
\hline Yes & $27(16.3)$ & $11(16.7)$ & $16(16.0)$ & \multirow{3}{*}{0.185} \\
\hline No & $105(63.3)$ & $37(56.1)$ & $68(68.0)$ & \\
\hline Unknown & $34(20.5)$ & $18(27.3)$ & $16(16.0)$ & \\
\hline \multicolumn{5}{|l|}{ History } \\
\hline Hysterectomy & $66(39.8)^{\mathrm{b}}$ & $66(100.0)^{\mathrm{b}}$ & $\mathrm{N} / \mathrm{A}$ & N/A \\
\hline POP/incontinence surg. & $67(40.4)$ & $54(81.8)$ & $13(13.0)$ & $<0.0005$ \\
\hline Intra-abdominal surg. & $68(41.0)^{c}$ & $36(54.5)^{\mathrm{c}}$ & $32(32.0)^{c}$ & 0.004 \\
\hline \multicolumn{5}{|l|}{ Pre-operative sPOPQ } \\
\hline sPOPQ Ba & $3(1-4)$ & $3(1-4)$ & $3(1-4)$ & 0.548 \\
\hline sPOPQ Bp & $2(1-4)$ & $2(1-4)$ & $1.5(1-4)$ & 0.035 \\
\hline sPOPQ C & $3(1-4)$ & $3(1-4)$ & $3(1-4)$ & 0.034 \\
\hline sPOPQ D & $2(1-4)$ & N/A & $2(1-4)$ & $\mathrm{N} / \mathrm{A}$ \\
\hline \multicolumn{5}{|l|}{ Concomitant surgery } \\
\hline Oophorectomyd & $9(5.4)$ & $2(3.0)$ & $7(7.0)$ & 0.320 \\
\hline $\mathrm{AC}$ & $15(9.0)$ & $4(6.1)$ & $11(11.0)$ & 0.277 \\
\hline $\mathrm{PC}$ & $2(1.2)$ & $0(0.0)$ & $2(2.0)$ & 0.518 \\
\hline Other & $5(3.0)$ & $3(4.5)$ & $2(2.0)$ & 0.650 \\
\hline Conversion & $2(1.2)$ & $2(3.0)$ & $0(0.0)$ & 0.157 \\
\hline
\end{tabular}

Data presented as number (\%), mean \pm SD or median (range).

$A C$ anterior colporrhaphy $A S A$ American Society of Anaesthesiologists $B M I$ body-mass index $N$ Number $P C$ posteriorcolporrhaphy $P O P$ Pelvic Organ Prolapse $S D$ standard deviation $S P O P Q$ simplified Pelvic Organ Prolapse Quantification RASC Robot-assisted laparoscopic sacrocolpopexy RSHS robot-assisted laparoscopic supracervical hysterectomy with sacrocervicopexy surg. Surgery $y r$ years

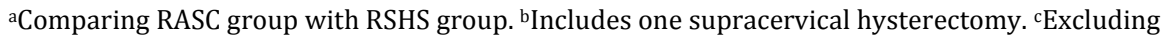
POP/incontinence surgery. dSingle or bilateral. 


\section{Mesh Exposure}

Two patients (1.2\%) were identified with mesh exposure, both $\leq 1 \mathrm{~cm}$ in diameter (Table 2 ). The incidences of mesh exposures after sacrocolpopexy and after supracervical hysterectomy and sacrocervicopexy were not significantly different [1/66 (1.5\%) and $1 / 100$ (1.0\%), respectively; $p=1.000]$.

Table 2. Overview of patients with mesh exposure

\begin{tabular}{|c|c|c|c|c|c|}
\hline $\begin{array}{l}\text { Previous } \\
\text { Surgery }\end{array}$ & $\begin{array}{l}\text { BMI } \\
\left(\mathrm{kg} / \mathrm{m}^{2}\right)\end{array}$ & $\begin{array}{l}\text { Associated. risk } \\
\text { factors }\end{array}$ & Procedure & Mesh exposure $^{a}$ & $\begin{array}{l}\text { Time to } \\
\text { exposure }(m)\end{array}$ \\
\hline None & 29.0 & $\begin{array}{l}\text { Smoking: no } \\
\text { PMP: yes } \\
\text { DM: no }\end{array}$ & RSHS & 2BT3S1 & 7.6 \\
\hline $\begin{array}{l}\text { Supracervical } \\
\text { hysterectomy }\end{array}$ & 21.6 & $\begin{array}{l}\text { Smoking: no } \\
\text { PMP: yes } \\
\text { DM: no }\end{array}$ & RASC & $\begin{array}{l}\text { 2AT3S1 } \\
\text { 2BcT4S1 }\end{array}$ & $\begin{array}{l}8.9 \\
33\end{array}$ \\
\hline \multicolumn{6}{|c|}{$\begin{array}{l}B M I \text { body-mass index DM Diabetes Mellitus } m \text { months } P M P \text { postmenopausal } R A S C \text { robot-assisted } \\
\text { laparoscopic sacrocolpopexy } R S H S \text { robot-assisted laparoscopic supracervical hysterectomy with } \\
\text { sacrocervicopexy }\end{array}$} \\
\hline
\end{tabular}

The first patient underwent a RSHS. She presented at 8 months after surgery with minimal vaginal and postcoital blood loss, without other complaints. Speculum examination revealed an exposition of the mesh (diameter, $0.5 \mathrm{~cm}$ ) on the posterior vaginal wall (2BT3S1), which was excised under local anesthetic after consent in the outpatient clinic. The vaginal wall was closed with Vicryl Rapide suture (Ethicon) and supplementation with vaginal estrogens was started. At follow-up, 13 months after removal, the patient exhibited no vaginal blood loss, no mesh exposure or prolapse. The second patient, with a history of a supracervical hysterectomy, underwent a RASC. Nine months postoperatively at routine follow-up, 2 sutures in the fornix posterior surrounded by granulation tissue were detected (2AT3S1). The sutures were removed at the outpatient clinic and the granulation tissue was treated with silver nitrate. Initially, this had the desirable effect, but 33 months after surgery she suffered from dyspareunia. A mesh exposure of $1 \mathrm{~cm}$ was now visible at the same location of the previous suture expositions (2BcT4S1). The mesh was excised in the outpatient clinic under local analgesia and treatment with vaginal estrogens was restarted. Further follow-up showed no recurrence. 


\section{Suture exposure and other mesh-related complications}

One patient $(0.6 \%)$ was seen 20 months after RASC with no complaints of exposure, but with urinary incontinence. On physical examination, one transmural suture surrounded by granulation tissue was visible at the top of the vagina. This suture was removed and silver nitrate was applied to treat the granulation tissue. Three patients $(1.8 \%)$ complained of vaginal pain during examination. One patient had severe atrophy and a suture was shimmering through the vaginal wall without epithelial separation. In the other two patients a prominence (i.e., a wrinkling or fold palpable without epithelial separation $)^{9}$ was found. Treatment with local estrogens was sufficient.

\section{Discussion}

To our knowledge, this is the largest prospective cohort study on mesh exposure after robot-assisted laparoscopic apical prolapse surgery reported to date. The low incidence of mesh exposure (1.2\%) is in line with previously published systematic reviews (23\%). ${ }^{3,4}$ More recent publications on exposure rates with a minimum follow-up duration of 12 months and a physical examination included, showed rates of $4.5 \%$ for RASC (18/401; range, $0-7.8 \%)^{11-18}$ and $1.4 \%$ for LSC (78/5755; range, $\left.0-21.4 \%\right) .{ }^{11-13,} 19-32$ The retrospective design of most of these studies and the high heterogeneity in definitions must be taken into account. Furthermore, some studies included concomitant total hysterectomy, which is associated with greater risk of mesh exposure. ${ }^{33} \mathrm{~A}$ well-designed prospective study of 143 patients showed no mesh exposures. ${ }^{34}$ Patients were objectively examined 1 year after RASC/RSHS and a lightweight type 1 polypropylene Ymesh (weight $33.5 \mathrm{~g} / \mathrm{m}^{2}$ ) was used. Kenton et al. ${ }^{13}$ conducted a randomized controlled trial (RCT) with an ultra-lightweight mesh but with Gore-Tex sutures, which showed no mesh exposure in either arm (RASC $n=33$; LSC, $n=33$ ). We chose to not change the type of mesh used (weight, $80-85 \mathrm{~g} / \mathrm{m}^{2}$ ), nor the (non-resorbable) suture type to evade heterogeneity. Further scientific evidence on lightweight mesh is scarce.

In studies with longer follow-up ( $\geq 3$ years) and a substantial number of patients $(\mathrm{n} \geq$ 50 ), mesh exposure occurred in $2.9 \%$ of RASC recipients (2/70; follow-up 72 months) ${ }^{14}$ and in $2.8 \%$ of LSC recipients (11/398; follow-up range, 43-60 months). ${ }^{23,28,35,36}$ In all five studies, type 1 polypropylene mesh was used. Nygaard et al. ${ }^{37}$ reported a high rate of mesh exposure after open abdominal sacrocolpopexy (10.5\% after 7 years follow-up). In this study, different types of mesh were used (Gore-Tex, Mersilene, biological material and type 1 polypropylene). Gore-Tex and Mersilene are associated with higher mesh 
exposure rates and biological material is associated with a high recurrence rate.38,39. Given the possible increase of mesh exposure over time, studies with longer follow-up and examining a single implant type are of major importance. Our study group is currently researching mesh exposure after longer follow-up periods.

The exact aetiology of mesh exposure remains unknown with contradicting evidence published. However, based on the literature, the difference between the transabdominal route and transvaginal route is apparent. Opening the vagina carries a theoretical risk of inducing infection of the graft due to contamination from vaginal microbes. ${ }^{39}$ Moreover, placing the mesh on newly created vaginal incisions could play a role in the occurrence of mesh exposure. ${ }^{40}$ The literature shows that this technique eventually results in high mesh exposure rates. ${ }^{2}$ In RASC and RSHS, the vaginal walls are not opened and only precise dissections with minimal tissue damage are made. Other risk factors associated with transvaginal mesh surgery are patient age, smoking, operative technique, surgeon experience, previous prolapse repair, concomitant hysterectomy, mesh properties and load, inverted T colpotomy incision, sexual activity, and diabetes. ${ }^{2}, 41,42$ Risk factors for abdominally placed mesh are more difficult to identify. The use of polytrafluroethylene (ePTFE) mesh, smoking, total hysterectomy (with opening of the vagina) or stage 3 or 4 prolapse have been reported. ${ }^{38,43}$ Even when the vaginal wall is left intact, it may be thin and atrophic, especially in elderly patients. Treatment with vaginal estrogens can possibly prevent exposure. The use of mesh in other techniques, such as minimal invasive sacral hysteropexy, also shows a low risk of mesh exposure. Gutman et al. ${ }^{44}$ reported a mesh exposure rate of $2.7 \%$ after laparoscopic sacral hysteropexy in their 1 year prospective parallel cohort.

Strengths of the present study are the use of standardized surgical procedures, inclusion of a single mesh type, and thorough examination with a specific transparent speculum. Detection of rare complications requires evaluation of large cohorts. RCT's, although considered the gold standard, are often too limited in size. Prospective trials have the benefit of including all patients (solid denominator) and examining a large population. ${ }^{45}$ Limitations of the study include the inclusion of solely tertiary referral hospitals for pelvic floor disorders. Some of the patients had complex pelvic floor disorders and/or an extensive history of pelvic floor surgery, which could possibly limit the generalizability of our findings. Another limitation is the loss to follow-up of 26 patients (13.5\%), who were not physically examined at 12 months postprocedure. Sixteen of these patients responded either by questionnaire or by telephone, reporting no mesh-related 
complaints (Figure 1). Finally, we examined patients who underwent sacrocolpopexy with and without a concomitant supracervical hysterectomy, which limited the homogeneity. However, in general practice it is commen to treat all posthysterectomy patients as patients with an intact uterus; therefore, both interventions were included in this study.

Comparing the abdominal use of mesh with literature on vaginally placed mesh, the abdominal route generated lower mesh exposure rates. These results are currently relevant owing to the public discussion on complications after mesh placement. Clear information about the safety or risk involved in the use of abdominal mesh has potential public health benefits by allowing doctors and patients to make informed decisions about the use of surgical mesh in prolapse surgery.

In conclusion, this large multicenter prospective cohort study shows a low incidence of mesh exposure after robot-assisted minimal invasive abdominal prolapse surgery. 


\section{References}

1. US Food and Drug Administration. Urogynecologic surgical mesh: update on the safety and effectiveness of transvaginal placement for pelvic organ prolapse. Available at: http://www.fda.gov/downloads/medicaldevices/safety/alertsandnotices/ucm262760.pdf Accessed January 15, 2017.

2. Abed H, Rahn DD, Lowenstein L, Balk EM, Clemons JL, Rogers RG. Incidence and management of graft erosion, wound granulation, and dyspareunia following vaginal prolapse repair with graft materials: A systematic review. Int Urogynecol J. 2011;22:789-798.

3. Serati M, Bogani G, Sorice P, Braga A, Torella M, Salvatore S, et al. Robot-assisted sacrocolpopexy for pelvic organ prolapse: a systematic review and meta-analysis of comparative studies. Eur Urol. 2014;66:303-318.

4. Lee RK, Mottrie A, Payne CK, Waltregny D. A review of the current status of laparoscopic and robot-assisted sacrocolpopexy for pelvic organ prolapse. Eur Urol. 2014;65:1128-1137.

5. Wu JM, Hundley AF, Fulton RG, Myers ER. Forecasting the prevalence of pelvic floor disorders in U.S. Women: 2010 to 2050. Obstet Gynecol. 2009;114:1278-1283.

6. Hubens G, Coveliers H, Balliu L, Ruppert M, Vaneerdeweg W. A performance study comparing manual and robotically assisted laparoscopic surgery using the da Vinci system. Surg Endosc. 2003:17:1595-1599.

7. Swift S, Morris S, McKinnie V, Freeman R, Petri E, Scotti RJ, et al. Validation of a simplified technique for using the POPQ pelvic organ prolapse classification system. Int Urogynecol J. Pelvic Floor Dysfunct. 2006;17:615-620. .

8. Parekh M, Swift S, Lemos N, Iskander M, Freeman B, Arunkalaivanan AS, et al. Multicenter interexaminer agreement trial for the validation of simplified POPQ system. Int Urogynecol J. 2011;22:645-650.

9. Haylen BT, Freeman RM, Swift SE, Cosson M, Davila GW, Deprest J, et al. An International Urogynecological Association (IUGA) / International Continence Society (ICS) joint terminology and classification of the complications related directly to the insertion of prostheses (meshes, implants, tapes) and grafts in female pelvic floor surgery. Int Urogynecol J. 2011;22:3-15.

10. Clifton MM, Pizarro-Berdichevsky J, Goldman HB. Robotic female pelvic floor reconstruction: a review. Urology. 2016;91:33-40.

11. Joubert M, Thubert T, Lefranc JP, Vaessen C, Chartier-Kastler É, Deffieux X, et al. Comparison of functional outcomes with purely laparoscopic sacrocolpopexy and robot-assisted sacrocolpopexy in obese women. Progrès en Urologie. 2014;24:1106-1113.

12. Tan-Kim J, Nager CW, Grimes CL, Luber KM, Lukacz ES, Brown HW, et al. A randomized trial of vaginal mesh attachment techniques for minimally invasive sacrocolpopexy. Int Urogynecol J. 2015;26:649-656.

13. Kenton K, Mueller ER, Tarney C, Bresee C, Anger JT. One-year outcomes after minimally invasive sacrocolpopexy. Female Pelvic Med Reconstr Surg. 2016;22:382-384.

14. Linder BJ, Chow GK, Elliott DS. Long-term quality of life outcomes and retreatment rates after robotic sacrocolpopexy. Int J Urol. 2015;22:1155-1158. 
15. Myers EM, Siff L, Osmundsen B, Geller E, Matthews CA. Differences in recurrent prolapse at 1 year after total vs supracervical hysterectomy and robotic sacrocolpopexy. Int Urogynecol J. 2015;26:585-589.

16. Jambusaria LH, Murphy M, Lucente VR. One-year functional and anatomic outcomes of robotic sacrocolpopexy versus vaginal extraperitoneal colpopexy with mesh. Female Pelvic Med Reconstr Surg. 2015;21:87-92.

17. Prendergast E, Silver H, Johnson LL, Simon M, Feinglass J, Kielb S, et al. Anatomic outcomes of robotic assisted supracervical hysterectomy and concurrent sacrocolpopexy at a tertiary care institution at initial adaptation of the procedure. Female Pelvic Med Reconstr Surg. 2016;22:29-32.

18. Linder BJ, Anand M, Klingele CJ, Trabuco EC, Gebhart JB, Occhino JA. Outcomes of robotic sacrocolpopexy using only absorbable suture for mesh fixation. Female Pelvic Med Reconstr Surg. 2017;23:13-16.

19. Culligan PJ, Salamon C, Priestley JL, Shariati A, Porcine dermis compared with polypropylene mesh for laparoscopic sacrocolpopexy: a randomized controlled trial. Obstet Gynecol. 2013;121:143151.

20. Rosati M, Bramante S, Bracale U, Pignata G, Azioni G. Efficacy of laparoscopic sacrocervicopexy for apical support of pelvic organ prolapse. JSLS. 2013;17:235-244.

21. Gracia M, Perelló M, Bataller E, Espuña M, Parellada M, Genís D, et al. Comparison between laparoscopic sacral hysteropexy and subtotal hysterectomy plus cervicopexy in pelvic organ prolapse : a pilot study. Neurourol Urodyn. 2015;34:654-658.

22. Park YH, Yang SC, Park ST, Park SH, Kim HB. Laparoscopic reconstructive surgery is superior to vaginal reconstruction in the pelvic organ prolapse. Int J Med Sci. 2014;11:1082-1088.

23. Sarlos D, Kots L, Ryu G, Schaer G. Long-term follow-up of laparoscopic sacrocolpopexy. Int Urogynecol J. 2014;25:1207-1212.

24. Liu C-K, Tsai CP, Chou MM, Shen PS, Chen GD, Hung YC, et al. A comparative study of laparoscopic sacrocolpopexy and total vaginal mesh procedure using lightweight polypropylene meshes for prolapse repair. Taiwan J Obstet Gynecol. 2014;53:552-558.

25. Li S, Ji M, Zhao Z. The effectiveness of two different laparoscopic surgeries for apical support of pelvic organ prolapse. Eur J Obstet Gynecol Reprod Biol. 2015;188:74-78.

26. El Hamamsy D, Fayyad AM. New onset stress urinary incontinence following laparoscopic sacrocolpopexy and its relation to anatomical outcomes. Int Urogynecol J. 2015;26:1041-1045.

27. Vieillefosse S, Thubert T, Dache A, Hermieu JF, Deffieux X. Satisfaction, quality of life and lumbar pain following laparoscopic sacrocolpopexy: suture vs. tackers. Eur J Obstet Gynecol Reprod Biol. 2015;187:51-56.

28. Costantini E, Mearini L, Lazzeri M, Bini V, Nunzi E, di Biase M, et al. Laparoscopic versus abdominal sacrocolpopexy: a randomized, controlled trial. J Urol. 2016;196:159-165.

29. Dandolu V, Akiyama M, Allenback G, Pathak P. Mesh complications and failure rates after transvaginal mesh repair compared with abdominal or laparoscopic sacrocolpopexy and to native tissue repair in treating apical prolapse. Int Urogynecol J. 2017:28:215-222.

30. Lizee D, Campagna G, Morciano A, Panico G, Ercoli A, Gadonneix P. Laparoscopic sacral colpopexy: how to place the posterior mesh into rectovaginal space? Neurourol Urodyn. 2017;36:1529-1435. 
31. Zebede S, Dawood A, Alarab M, Drutz H, Lovatsis D. A streamlined surgical approach to laparoscopic sacrocolpopexy for post-hysterectomy vault prolapse. J Obstet Gynaecol Can. 2016;38:446-452.

32. Chen Y, Hua K. Medium-term outcomes of laparoscopic sacrocolpopexy or sacrohysteropexy versus vaginal sacrospinous ligament fixation for middle compartment prolapse. Int J Gynecol Obstet. 2017;137:164-169.

33. Osmundsen BC, Clark A, Goldsmith C, Adams K, Denman MA, Edwards R, et al. Mesh erosion in robotic sacrocolpopexy. Female Pelvic Med Reconstr Surg. 2012;18:86-88.

34. Culligan PJ, Gurshumov E, Lewis C, Priestly JL, Komar J, Shah N, et al. Subjective and objective results 1 year after robotic sacrocolpopexy using a lightweight Y-mesh. Int Urogynecol J. 2014;25:731-735.

35. Granese R, Candiani M, Perino A, Romano F, Cucinella G. Laparoscopic sacrocolpopexy in the treatment of vaginal vault prolapse: 8 years experience. Eur J Obstet Gynecol Reprod Biol. 2009;146:227-231.

36. Sabbagh R, Mandron E, Piussan J, Brychaert PE, Tule M. Long-term anatomical and functional results of laparoscopic promontofixation for pelvic organ prolapse. BJU Int. 2010;106:861-866.

37. Nygaard I, Brubaker L, Zyczynski HM, Cundiff G, Richter H, Gantz M, et al. Long-term outcomes following abdominal sacrocolpopexy for pelvic organ prolapse. JAMA. 2013;309:2016-2024.

38. Cundiff GW, Varner E, Visco AG, Zyczynski HM, Nager CW, Norton PA, et al. Risk factors for mesh/suture erosion following sacral colpopexy. Am J Obstet Gynecol. 2008;199:688.e1-688.e5.

39. Nygaard IE, McCreery R, Brubaker L, Connolly A, Cundiff G, Weber AM, et al. Abdominal sacrocolpopexy: a comprehensive review. Obstet Gynecol. 2004;104:805-823.

40. Bensinger G, Lind L, Lesser M, Guess M, Winkler HA. Abdominal sacral suspensions: analysis of complications using permanent mesh. Am J Obstet Gynecol. 2005;193:2094-2098.

41. Collinet P, Belot F, Debodinance P, Ha Duc E, Lucot JP, Cosson M. Transvaginal mesh technique for pelvic organ prolapse repair: mesh exposure management and risk factors. Int Urogynecol J. 2006;17:315-320.

42. Miklos JR, Chinthakanan O, Moore RD, Mitchell GK, Favors S, Karp DR, et al. The IUGA/ICS classification of synthetic mesh complications in female pelvic floor reconstructive surgery: a multicenter study. Int Urogynecol J. 2016;27:933-938.

43. Akyol A, Akca A, Ulker V, Gedikbasi A, Kublay A, Han A, et al. Additional surgical risk factors and patient characteristics for mesh erosion after abdominal sacrocolpopexy. J Obstet Gynaecol Res. 2014;40:1368-1374.

44. Gutman RE, Rardin CR, Sokol ER, Matthews C, Park AJ, Iglesia CB, et al. Vaginal and laparoscopic mesh hysteropexy for uterovaginal prolapse: a parallel cohort study. Am J Obstet Gynecol. 2017;216:38.e1-38e11.

45 Tamussino K, Falconer C, Schraffordt Koops SE, Nilsson CG. Comment on the editorial by Roovers : "Registries: what level of evidence do they provide ?" Int Urogynecol J. 2008;19:1585. 




\section{Chapter 8}

Long-term mesh erosion rate following abdominal robotic reconstructive pelvic floor surgery: a prospective study and overview of the literature

Femke van Zanten, Jan J. van lersel, Tim J.C. Paulides, Paul M. Verheijen, Ivo A.M.J. Broeders, Esther C.J. Consten Egbert Lenters, Steven E. Schraffordt Koops 


\section{Abstract}

Introduction and Hypothesis: The use of synthetic mesh in transvaginal pelvic floor surgery has been subject to debate internationally. Although mesh erosion appears to be less associated with an abdominal approach, the long-term outcome has not been studied intensively. This study was set up to determine the long-term mesh erosion rate following abdominal pelvic reconstructive surgery.

Methods: A prospective, observational cohort study, conducted in a tertiary care setting. All consecutive female patients who underwent robot-assisted laparoscopic sacrocolpopexy and sacrocolporectopexy in 2011 and 2012 were included. Primary outcome was mesh erosion. Preoperative and postoperative evaluation (6 weeks, 1 year, 5 years) with a clinical examination and questionnaire regarding pelvic floor symptoms was performed. Mesh-related complications were assessed using a transparent vaginal speculum, proctoscopy, and digital vaginal and rectal examination. Kaplan-Meier estimates were calculated for mesh erosion. A review of the literature on mesh exposure after minimally invasive sacrocolpopexy was performed ( $\geq 12$ months' follow-up).

Results: Ninety-six of the 130 patients included (73.8\%) were clinically examined. Median follow-up time was 48.1 months (range 36.0-62.1). Three mesh erosions were diagnosed (3.1\%; Kaplan-Meier 4.9\%, 95\% confidence interval 0-11.0); one bladder erosion for which mesh resection and an omental patch interposition were performed, and two asymptomatic vaginal erosions (at 42.7 and 42.3 months) treated with estrogen cream in one. Additionally, 22 patients responded solely by questionnaire and/or telephone, none reported mesh-related complaints. The literature, mostly based on retrospective studies, described a median mesh erosion rate of 1.9\% (range 0-13.3\%).

Conclusions: The long-term rate of mesh erosion following an abdominally placed synthetic graft is low. 


\section{Introduction}

The use of synthetic mesh in pelvic floor surgery has been subject to debate. In 2008 and 2011, the US Food and Drug Administration (FDA) warned about the high rate of meshrelated complications following transvaginal pelvic organ prolapse repair. ${ }^{1}$ The FDA warnings were underlined by a systematic review reporting an incidence of mesh erosion of $10.3 \%$ (range $0-29.7 \%, n=11,785$ ) following transvaginal pelvic organ prolapse repair in the first postoperative year. ${ }^{2}$ Recent literature on transvaginal repair has confirmed this high incidence. ${ }^{3}$ Transabdominal approaches for pelvic reconstructive surgery are associated with a much lower incidence of mesh erosion.1,4 However, most studies describing mesh erosion are retrospective with short-term follow-up. Research focusing specifically on long-term mesh-related morbidity is lacking.

Minimally invasive sacrocolpopexy is currently the preferred treatment for apical prolapse, and ventral mesh rectopexy has gained increasing worldwide acceptance for rectal prolapse.5,6 More recently, the two abdominal procedures combined have been described and are being used as a treatment for combined pathology.7,8

It is against this backdrop that we designed a study to evaluate the long-term mesh erosion rate following robot-assisted laparoscopic sacrocolpopexy (RASC) and robotassisted laparoscopic sacrocolporectopexy (RSCR). Second, we performed a literature review on mesh erosion after minimally invasive sacrocolpopexy with a minimal followup duration of 12 months.

\section{Materials and Methods}

\section{Study design and participants}

All consecutive female patients who underwent RASC or RSCR at a tertiary referral centre for pelvic floor disorders in the Netherlands in 2011 and 2012, were prospectively included. The set-up was an observational cohort study. The primary outcome was long-term mesh erosion. 


\section{Inclusion and exclusion criteria}

Inclusion criteria were patients with symptomatic vaginal vault prolapse [simplified pelvic organ prolapse quantification (SPOPQ) stage $\geq 2$ ] and patients with additional symptomatic internal/external rectal prolapse (Oxford Grading System grade $\geq 3$; an additional enterocele or rectocele may be present). Exclusion criteria were conversion to another procedure without mesh usage, poor health status with inability to undergo general anesthesia, patients younger than 18 years, $\geq 3$ previous laparotomic surgeries, planned pregnancy, known pelvic/abdominal malignancies. This study was carried out in accordance with the ethical standards of the Central Committee on Research Involving Human Subjects and with the Declaration of Helsinki. Patients gave informed consent before inclusion.

\section{Clinical evaluation}

Patients were clinically reviewed preoperatively and postoperatively at 6 weeks, 1 year, 5 years and in cases where complaints occurred. Rectal prolapse was diagnosed and evaluated at follow-up using the Oxford Grading System by proctoscopy and dynamic MRI. ${ }^{9}$ The SPOPQ was used to determine vaginal prolapse. ${ }^{10}$ At follow-up, all patients underwent a digital vaginal and rectal examination, a proctoscopy and a vaginal speculum examination to assess mesh-related complications. Both proctoscope and speculum were transparent. Patients were examined in the supine lithotomy position using leg supports, both in rest and during maximal Valsalva. Clinical examination was performed by an objective researcher (not blinded). If mesh-related morbidity was suspected, a second examination by a gynecologist was performed to confirm the diagnosis. Mesh erosion was graded according to the International Urogynecological Association (IUGA) and the International Continence Society (ICS) joint terminology and category, time and site (CTS) classification, although we used the term mesh erosion instead of mesh exposure. ${ }^{11}$ During every evaluation (pre- and postoperatively), patients received a surgical and urogynecological questionnaire on paper, which included questions regarding symptoms of bulge, micturition (Urinary Distress Inventory; UDI-6), defecation (obstructive defecation and fecal incontinence) and quality of life (Pelvic Floor Impact Questionnaire; PFIQ-7). ${ }^{12,13}$ In case patients declined clinical evaluation, patients were invited to return the questionnaire by mail. Questions regarding meshrelated morbidity were asked postoperatively during the clinical evaluation or, in case patients declined examination, by telephone: 'vaginal/rectal bleeding or discharge', 'vaginal/rectal pain', 'pelvic pain (either spontaneous or during physical activity)', 
'recurrent urinary tract infection'. Patients were considered lost to follow-up in cases where no physical examination or no questionnaire was available. Solely patients with a postoperative physical examination available were included in the analysis to determine the mesh erosion rate.

\section{Surgical technique}

All procedures were performed using the da Vinci robot (Intuitive Surgical, Sunnyvale, CA, USA) by 3 colon surgeons and 2 urogynecologists with $\geq 10$ years' experience. Every patient received prophylactic intravenous antibiotics $(1,000 \mathrm{mg}$ cefazolin and $500 \mathrm{mg}$ metronidazole) 15 minutes prior to incision. The RASC procedures, with or without supracervical hysterectomy, were performed according to the technique described by Clifton et al. ${ }^{14}$ RASC was performed solely by the gynecologist. The technique of RSCR was performed jointly by one colorectal and one urogynecological surgeon. The technique of RSCR has been previously described by our study group. ${ }^{7}$ All meshes (type 1, macroporous polypropylene, Prolene, Ethicon Inc., Johnson \& Johnson, Hamburg, Germany, weight $80-85 \mathrm{~g} / \mathrm{m}^{2}$ ) were distally attached using non-absorbable sutures (Ethibond; Ethicon, Johnson \& Johnson, Hamburg, Germany) and anchored proximally to the sacral promontory using titanium tacks (Autosuture Protack 5mm; Covidien, USA). Two meshes were used, configured into a ' $\mathrm{Y}$ ' shape intracorporeally. The peritoneum was approximated to cover the implant using a $23 \mathrm{~cm}$ V-Loc suture (Covidien, Mansfield, MA, USA). The surgeon performed a vaginal/rectal examination at the end of each procedure to exclude a possible (suture) perforation of the vaginal and/or rectal wall and to determine the correct position of the mesh.

\section{Review of the literature}

To compare our mesh erosion rate with the literature, a literature search was performed describing mesh erosion after minimally invasive sacrocolpopexy with a minimal duration of follow-up of 12 months (Appendix A). Studies describing mesh erosion after open/minimally invasive sacrocolporectopexy were described separately (Appendix A).

\section{Statistical Analysis}

Statistical Package for the Social Sciences, version 20.0 (IBM, Armonk, NY, USA) was used for statistical analysis. Data are presented as percentage, mean \pm standard deviation (SD), and median and range. Chi-squared test, Fisher's exact test and independent sample $\mathrm{t}$ test were used to compare variables between groups for 
categorical and continues data. Because of differences in follow-up between patients, the Kaplan-Meier method with 95\% confidence intervals was used to estimate the erosion rate at various time points. The Log-Rank Test was used to compare Kaplan-Meier estimates between subgroups.

Figure 1. Flowchart of patients included.

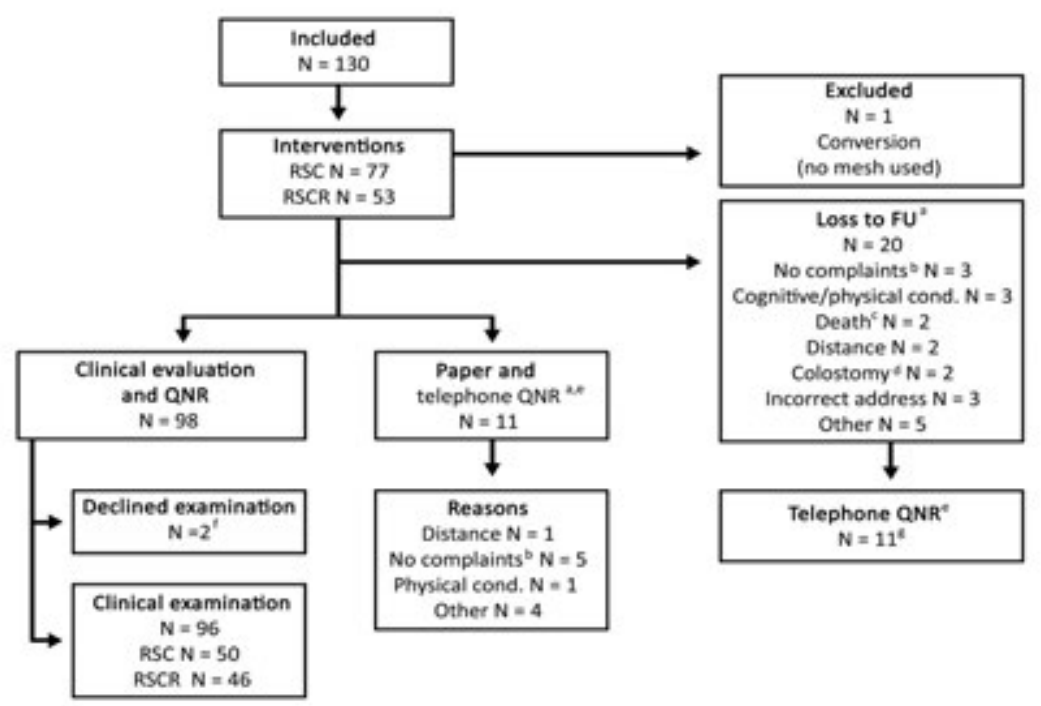

cond. condition $F U$ follow-up $N$ number $R S C$ robot-assisted sacrocolpopexy $R S C R$ robot-assisted sacrocolporectopexy QNR questionnaire

aThe general practitioner was contacted in the case of incorrect address details. bPatients had no complaints and patients themselves judged an examination to be unnecessary. 'Due to natural causes.

dTwo patients received a colostomy and declined further participation: one because of therapyresistant fecal incontinence and extensive sphincter dysfunction, and one because of disabling obstructed defecation. eQuestionnaires regarding pelvic floor symptoms. The paper questionnaire was discussed during consultation. Patients who were unable to attend or declined clinical examination were asked to send back the questionnaire by post. These patients and patients who were lost to follow-up were contacted additionally by telephone to ask for specific anamnestic mesh-related

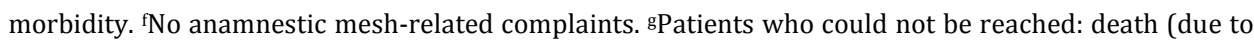
natural causes) $n=2$, cognitive/physical condition $n=3$, untraceable $n=3$, other $n=1$. 


\section{Results}

\section{Patients}

One hundred and thirty patients underwent surgery. One procedure $(0.8 \%)$ was converted to vaginal prolapse surgery owing to a pre-sacral bleeding. This patient was excluded from the study, since no mesh was placed. Twenty patients (15.4\%) were lost to follow-up and 11 patients (8.5\%) solely responded by questionnaire. In total, 96 patients $(73.8 \%)$ were physically examined in the outpatient clinic. The flow chart of patients included is shown in Figure 1.

\section{Demographics and operative data}

Fifty women (52.1\%) underwent an RASC and 46 women (47.9\%) an RSCR (Table 1). RASC and RSCR were combined with a concomitant supracervical hysterectomy in 36 $(72 \%)$ and in $25(54.3 \%)$ cases respectively. Mean age and body mass index of all patients were $62.3 \pm 10.4$ years and $26.1 \pm 4.2 \mathrm{~kg} / \mathrm{m} 2$ respectively. Two cases $(2.1 \%)$ were converted to an open procedure (extensive intra-abdominal adhesions $n=1$; anesthesia-related issues $n=1)$. Intra-operative complications occurred in $3(3.1 \%)$ patients; 2 small bladder perforations in the bladder dome and 1 minor serosal small bowel lesion. No (mesh-related) postoperative complications were observed in these specific patients. Median follow-up time was 48.1 months (range $36.0-62.1$ ). 
Table 1. Patient demographics and operative data

\begin{tabular}{|c|c|c|c|c|}
\hline & $\begin{array}{l}\text { Total } \\
\mathrm{N}=96\end{array}$ & $\begin{array}{l}\text { RASC } \\
n=50^{a}\end{array}$ & $\begin{array}{l}\text { RSCR } \\
n=46\end{array}$ & $p$ value \\
\hline Mean age (SD) & $62.3(10.4)$ & $62.4(9.5)$ & $62.2(11.5)$ & 0.922 \\
\hline Mean ASA classification (SD) & $1.8(0.5)$ & $1.7(0.5)$ & $1.9(0.5)$ & 0.112 \\
\hline Mean parity (SD) & $2.8(1.0)$ & $2.8(1.1)$ & $2.8(1.0)$ & 0.898 \\
\hline Mean BMI (SD) & $26.1(4.2)$ & $25.9(3.7)$ & $26.3(4.7)$ & 0.683 \\
\hline Episiotomy (\%) & $51(53.1)$ & $29(58.0)$ & $22(47.8)$ & 0.318 \\
\hline Prolapse $1^{\text {st }}$ degree relative (\%) & 35 (36.5) & $20(40.0)$ & $15(32.6)$ & 0.648 \\
\hline Smoking (\%) & $23(24.0)$ & $12(24.0)$ & $11(23.9)$ & 0.957 \\
\hline Sexually active (\%) & 45 (46.9) & $25(50.0)$ & $20(43.5)$ & 0.198 \\
\hline \multicolumn{5}{|l|}{ History (\%) } \\
\hline TVT & $5(5.2)$ & $1(2.0)$ & $4(8.7)$ & $0.195^{\mathrm{b}}$ \\
\hline Burch colposuspension & $1(1.0)$ & $1(2.0)$ & 0 & $1.000^{\mathrm{b}}$ \\
\hline Hysterectomy & $34(35.4)$ & $14(28.0)$ & $20(43.5)$ & 0.113 \\
\hline Sacrocolpopexy & $1(1.0)$ & $1(2.0)$ & 0 & $1.000^{\mathrm{b}}$ \\
\hline Anterior colporrhaphy & $20(20.8)$ & $9(18.0)$ & $11(23.9)$ & 0.476 \\
\hline Posterior colporrhaphy & 19 (19.8) & $8(16.0)$ & $11(23.9)$ & 0.331 \\
\hline Rectopexy & $2(2.1)$ & $1(2.0)$ & $1(2.2)$ & $1.000^{\mathrm{b}}$ \\
\hline Perineal procedure & $2(2.1)$ & 0 & $2(4.3)$ & $0.227^{b}$ \\
\hline Sphincter procedure & 0 & 0 & - & $\mathrm{N} / \mathrm{A}$ \\
\hline Hemorrhoidectomy & $2(2.1)$ & 0 & $2(4.3)$ & $0.227^{\mathrm{b}}$ \\
\hline Other abdominal surgery & $32(33.3)$ & $15(30.0)$ & $17(37.0)$ & 0.470 \\
\hline \multicolumn{5}{|l|}{ Rectal prolapse (\%) } \\
\hline ERP & $4(4.2)$ & 0 & $4(8.7)$ & $0.049 \mathrm{~b}$ \\
\hline IRP or/and symp. rectocele* & $49(51.0)$ & $21(42.0)$ & $28(60.9)$ & 0.065 \\
\hline${ }^{*}$ with enterocele & $15(15.6)$ & $3(6.0)$ & $12(26.1)$ & 0.007 \\
\hline \multicolumn{5}{|l|}{ sPOPQ, mean (SD) } \\
\hline sPOPQ Ba & $2.4(1.0)$ & $2.6(0.9)$ & $2.4(0.9)$ & 0.947 \\
\hline sPOPQ Bp & $1.9(1.0)$ & $1.9(1.0)$ & $2.2(1.0)$ & 0.149 \\
\hline sPOPQ C & $2.5(1.0)$ & $2.9(0.9)$ & $2.3(1.0)$ & 0.021 \\
\hline sPOPQ D & $2.0(1.0)$ & $2.4(1.0)$ & $2.2(1.0)$ & 0.273 \\
\hline \multicolumn{4}{|l|}{ hysterectomy (\%) } & 0.073 \\
\hline Conversion (\%) & $2(2.1)$ & $1(2.0)$ & $1(2.2)$ & $1.000^{\mathrm{b}}$ \\
\hline Intra-operative complications (\%) & $3(3.1)$ & 0 & $3(6.5)$ & 0.106 \\
\hline Mean LOS, nights (SD) & $2.8(1.2)$ & $2.3(0.9)$ & $3.4(1.2)$ & $<0.0005$ \\
\hline
\end{tabular}


Table 1. Continued

\begin{tabular}{|c|c|c|c|c|}
\hline & $\begin{array}{l}\text { Total } \\
\mathrm{N}=96\end{array}$ & $\begin{array}{l}\text { RASC } \\
\mathrm{n}=\mathbf{5 0}^{\mathrm{a}}\end{array}$ & $\begin{array}{l}\text { RSCR } \\
\mathrm{n}=46\end{array}$ & $p$ value \\
\hline \multicolumn{5}{|l|}{ Early postoperative complications (\%) } \\
\hline CD grade $\leq 2$ & $2(2.1)$ & $2(4.0)$ & 0 & $0.496^{\mathrm{b}}$ \\
\hline $\mathrm{CD}$ grade $\geq 3$ & $1(1.0)$ & $1(2.0)$ & 0 & $1.000^{\mathrm{b}}$ \\
\hline Mesh erosion (\%) & $3(3.1)$ & $2(4.0)$ & $1(2.2)$ & $1.000^{\mathrm{b}}$ \\
\hline Postoperative in-hospital mortality (\%) & 0 & 0 & 0 & $\mathrm{~N} / \mathrm{A}$ \\
\hline \multicolumn{5}{|c|}{$\begin{array}{l}A S A \text { American Society of Anesthesiologists } B M I \text { body mass index } C D \text { Clavien-Dindo classification } E R P \\
\text { external rectal prolapse } I R P \text { internal rectal prolapse } L O S \text { length of hospital stay } N / A \text { not applicable } \\
R A S C \text { robot-assisted sacrocolpopexy } R S C R \text { robot-assisted sacrocolporectopexy } S D \text { standard deviation } \\
\text { symp. Symptomatic } S P O P Q \text { simplified pelvic organ prolapse quantification } T V T \text { Tension-free vaginal } \\
\text { tape a2 RASC were combined with a TVT. bFisher's Exact Test. }\end{array}$} \\
\hline
\end{tabular}

\section{Mesh-related complications}

Three patients (3.1\%) developed mesh erosion during follow-up (Table 2). The accompanying actuarial erosion rates for the total cohort were $0 \%$ after 1 year, $0 \%$ after 3 years, and $4.9 \%$ after 5 years (95\% confidence interval 0-11.0; Figure 2; Kaplan-Meier curve). The Kaplan Meier estimates for RASC and RSCR after 5 years were 5.3 (95\% CI 0$12.4 \%$ ) and 3.0 (95\% CI 0-8.9) respectively. No significant difference between the two subgroups could be found $(\mathrm{p}=0.808)$. The first patient presented with pain, dysfunctional voiding and recurrent urinary tract infections 45 months after RASC with supracervical hysterectomy. A small defect of the posterior wall of the bladder with mesh exposure was observed with cystoscopy. The mesh was removed and an omental patch interposition was performed. The second mesh erosion was discovered during regular follow-up 42.7 months following RSCR with supracervical hysterectomy. An asymptomatic erosion was found in the posterior wall of the vagina for which vaginal estrogen was prescribed. The third mesh erosion was also asymptomatic and was found in the posterior wall of the vagina at 42.3 months after RASC. Since the mesh exposure was so small, expectant management was chosen. All three patients who developed a mesh erosion had an extensive surgical pelvic floor history (Table 2). Two of the three women were postmenopausal. The three patients with mesh erosion had some of the characteristics and recognized risk factors for mesh erosion, including history of pelvic floor surgery $(n=3)$, vaginal atrophy $(n=3)$, smoking $(n=1)$, sexual activity $(n=1)$ and age $>70$ years $(n=2)$. During the intraoperative vaginal examination of one of these patients, a perforating suture was removed, which may be another risk factor for the occurrence of mesh erosion. 
Figure 2. Kaplan Meier Curve of mesh erosion

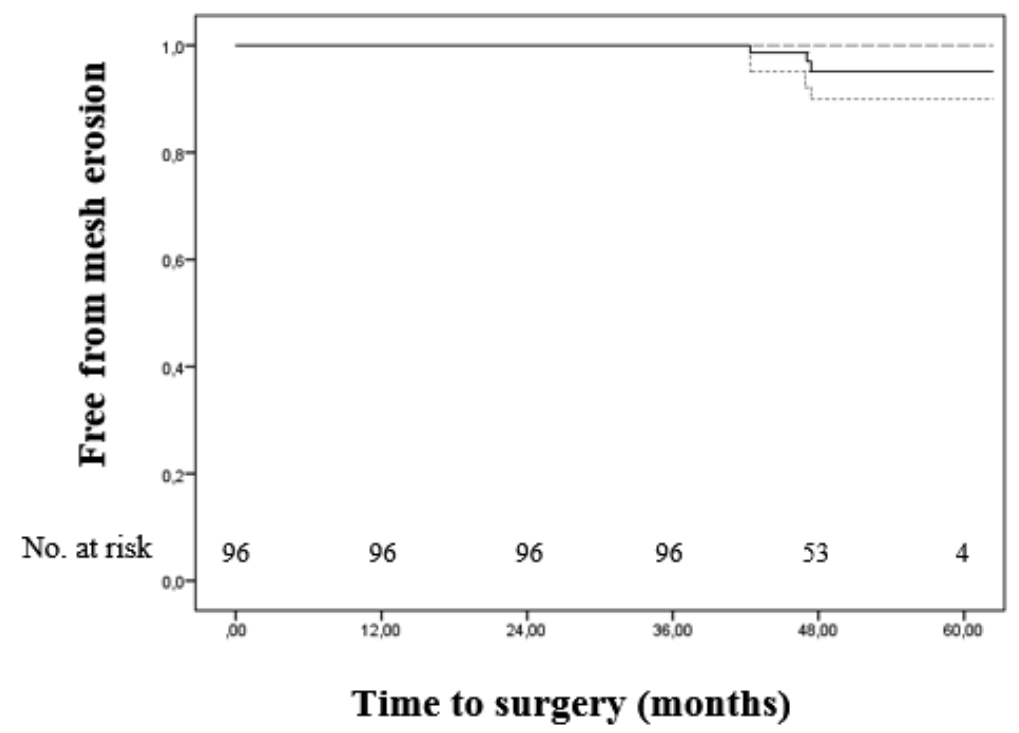

Kaplan-Meier curve for mesh erosion after RASC and RSCR (straight line). Dotted grey lines represent upper and lower $95 \%$ confidence intervals. The duration of event-free survival was measured from the date of surgery to the time of the event (complete) or the last follow-up (censored).

Four (4.2\%) other post-menopausal patients (mean age $70.3 \pm 7.8$ years), all with vaginal atrophy, experienced vaginal discomfort examining the distal side of the mesh. No mesh erosion or other mucosal abnormalities were observed. One of these patients developed postoperative new-onset dyspareunia, but declined the use of vaginal estrogens, because of the sporadic occurrence of complaints. All four patients were offered vaginal estrogen therapy, only two (both sexually active) patients accepted the prescription. No other mesh-related morbidity was observed in the complete cohort. Twenty-two patients were assessed solely by questionnaire; none reported meshrelated complaints.

\section{Overview of literature}

Details of the literature search and a flow-chart of studies included are presented in Appendix A. Sixty-five studies described mesh erosion after laparoscopic sacrocolpopexy (LSC) and/or RASC (Table 3). Most studies were of a retrospective design (73.8\%). The literature on LSC and RASC shows erosion rates between 0 and 13.3\% (range of number of patients included: 12-4,552; range of follow-up $12-72$ months).3,5,15-77 The articles that were included differed in their methods and inclusion 
criteria. Some studies solely included post hysterectomy patients. Other studies also included patients with their uterus still present, performing either a total hysterectomy or supracervical hysterectomy. Furthermore, different types of mesh were used throughout the studies. Eighty-three percent of the articles reported an erosion percentage of $\leq 5 \%$ with an overall median erosion rate of $1.9 \%$. Six studies $(9.2 \%)$ had a follow-up duration of more than 48 months $24,34,43,51,61,74$ One of these six studies included 391 patients. The authors reported mesh-related complications requiring surgical intervention in $2.8 \% .^{51}$ However, follow-up in this study, was carried out by telephone interview and the numbers could therefore be underestimated. The other five studies reported on 361 patients in total, with 15 mesh erosions (4.1\%; range of mesh erosion 2.9-7.8\%). This is in line with the mesh erosion rate found in our study. Dandolu et al. ${ }^{3}$ described a large retrospective cohort of patients ( $N=4,552$, follow-up $\geq 2$ years) with an apical prolapse who underwent LSC. Mesh removal or revision occurred in 52 patients (1.7\%). One study of Geller et al. ${ }^{59}$ reported a mesh erosion rate of $13 \%$. The study included solely 15 patients, which could possibly explain the high erosion rate. Practically all erosions reported in the literature were symptomatic. One study mentions asymptomatic mesh erosion. ${ }^{62}$ Three studies on sacrocolpopexy using a lightweight mesh show an erosion percentage of $0 \%$ after 12 months of follow-up. $41,68,71$

Table 2. Mesh erosions in the current study

\begin{tabular}{|c|c|c|c|c|c|c|c|}
\hline $\begin{array}{l}\text { Age, } y \\
\text { (ASA) }\end{array}$ & $\begin{array}{l}\text { Surgical } \\
\text { history }\end{array}$ & Procedure & $\begin{array}{l}\text { Location, } \\
\text { symptoms }\end{array}$ & CTS $^{11}$ & Defect & $\begin{array}{l}\text { Mo. to } \\
\text { erosion }\end{array}$ & Treatment \\
\hline $50(2)$ & $\begin{array}{l}\text { Cervical } \\
\text { amputation, } \\
\text { VMR, AC, PC }\end{array}$ & $\begin{array}{l}\text { RASC with } \\
\text { supracervical } \\
\text { hysterectomy }\end{array}$ & $\begin{array}{l}\text { Bladder, } \\
\text { posterior wall, } \\
\text { symptomatic }\end{array}$ & 4B/T4/S3 & $<1 \mathrm{~cm}$ & 45.0 & $\begin{array}{l}\text { Mesh resection, } \\
\text { omental patch } \\
\text { interposition }\end{array}$ \\
\hline 77 (2) & $\begin{array}{l}\text { Unknown } \\
\text { prolapse } \\
\text { surgery, } \\
\text { AC, PC }\end{array}$ & $\begin{array}{l}\text { RSCR with } \\
\text { supracervical } \\
\text { hysterectomy }\end{array}$ & $\begin{array}{l}\text { Vagina, } \\
\text { posterior wall, } \\
\text { asymptomatic }\end{array}$ & 2A/T4/S1 & $1 \mathrm{~cm}$ & 42.7 & $\begin{array}{l}\text { Vaginal } \\
\text { estrogen } \\
\text { therapy, twice a } \\
\text { week }\end{array}$ \\
\hline $74(2)$ & $\begin{array}{l}\text { Hysterec- } \\
\text { tomy, PC } \\
\text { and McCall }\end{array}$ & RASC & $\begin{array}{l}\text { Vagina, } \\
\text { posterior wall, } \\
\text { asymptomatic }\end{array}$ & 2A/T4/S1 & $<1 \mathrm{~cm}$ & 42.3 & $\begin{array}{l}\text { Expectant } \\
\text { management }\end{array}$ \\
\hline
\end{tabular}

$A C$ anterior colporrhaphy ASA American Society of Anesthesiologists CTS categorie (C) time (T) and site (S) mo. Months PC posterior colporrhaphy RASC robot-assisted sacrocolpopexy RSCR robotassisted sacrocolporectopexy y years $V M R$ ventral mesh rectopexy 
Four studies described mesh erosion after open/minimal invasive sacrocolporectopexy, varying from 2.0 to $5.4 \%$ (median range of follow-up 195 days - 64 months).7,8,78,79 Only 1 of the 4 studies performed a rectal and vaginal examination after 12 months of followup and noted a $2 \%$ erosion rate. ${ }^{7}$

Table 3. Mesh erosion following minimally invasive sacrocolpopexy with synthetic mesh ( $\geq$ mo FU)

\begin{tabular}{|c|c|c|c|c|c|c|}
\hline First author & $\begin{array}{l}\text { No. of } \\
\text { patients }\end{array}$ & $\begin{array}{l}\text { Material } \\
\text { and type } \\
\text { of mesh }\end{array}$ & $\begin{array}{l}\text { Vaginal/ } \\
\text { rectal } \\
\text { exam }\end{array}$ & $\begin{array}{l}\text { Follow-up } \\
\text { mo } \\
\text { (median) }\end{array}$ & $\begin{array}{l}\text { Mesh } \\
\text { compl. } \\
(\%)\end{array}$ & $\begin{array}{l}\text { Mesh } \\
\text { erosion } \\
(\%)\end{array}$ \\
\hline \multicolumn{7}{|l|}{ Laparoscopic and Robotic } \\
\hline Paraiso et al..$^{5} 2011$ & $\begin{array}{l}33 L \\
35 R\end{array}$ & $\mathrm{PP}, 1$ & Only vag. & 12 & $\begin{array}{l}0 L \\
2^{\mathrm{a}}(5.7) R\end{array}$ & $\begin{array}{l}0 L, 2^{\text {a }} \\
(5.7) R\end{array}$ \\
\hline Chan et al..$^{15} 2011$ & $\begin{array}{l}20 \mathrm{~L}, \\
16 R\end{array}$ & $\mathrm{PP}, 1$ & Only vag. & $\begin{array}{l}39 L \\
16 R\end{array}$ & $\begin{array}{l}0 L \\
0 R\end{array}$ & $\begin{array}{l}0 L \\
0 R\end{array}$ \\
\hline Tan-Kim et al. ${ }^{16} 2011$ & $\begin{array}{l}58 \mathrm{~L} \\
41 R\end{array}$ & $\mathrm{PP}, 1$ & Only vag. & $\begin{array}{l}12 L \\
19 R\end{array}$ & $\begin{array}{l}2(3.6) L \\
2(4.9) R\end{array}$ & $\begin{array}{l}2(3.6) L \\
2(4.9) R\end{array}$ \\
\hline Seror et al. ${ }^{17} 2012$ & $\begin{array}{l}47 \mathrm{~L} \\
20 R\end{array}$ & $\mathrm{PP}, 1$ & Only vag. & $\begin{array}{l}18 L \\
15 R\end{array}$ & $\begin{array}{l}1(2.1) L \\
0 R\end{array}$ & $\begin{array}{l}1(2.1) L \\
0 R\end{array}$ \\
\hline Joubert et al. ${ }^{18} 2014$ & $\begin{array}{l}39 \mathrm{~L}, \\
17 R\end{array}$ & $\begin{array}{l}\mathrm{PP}, 1 / \\
\mathrm{PE}, 3\end{array}$ & Only vag. & $\begin{array}{l}14.9 \\
12 R\end{array}$ & $\begin{array}{l}2(5.1) L \\
0 R\end{array}$ & $\begin{array}{l}2(5.1) L \\
0 R\end{array}$ \\
\hline Tan-Kim et al. ${ }^{19} 2015$ & $\begin{array}{l}32 L \\
32 R\end{array}$ & $\mathrm{PP}, 1$ & Only vag. & 12 & $\begin{array}{l}1(3.1) L \\
2(6.3) R\end{array}$ & $\begin{array}{l}1(3.1) L \\
1(3.1) R\end{array}$ \\
\hline Kenton et al..$^{20} 2016$ & $33 L, 33 R$ & $\mathrm{PP}, 1$ & Only vag. & 12 & 0 & 0 \\
\hline Laparoscopic & & & & & & \\
\hline Antiphon et al..$^{21} 2004$ & 104 & $\mathrm{PE}, 3$ & Only vag. & 17 & $2(1.9)$ & 0 \\
\hline Gadonneix et al. ${ }^{22} 2004$ & 46 & $\mathrm{PE}, 3$ & Only vag. & 24 & 0 & 0 \\
\hline Paraiso et al. ${ }^{23} 2005$ & 56 & $\mathrm{PP}, 1$ & $\mathrm{n} / \mathrm{d}$ & $13.5^{\mathrm{b}}$ & $2(3.6)$ & $2(3.6)$ \\
\hline Ross et al. ${ }^{24} 2005$ & 51 & $\mathrm{PP}, 1$ & Only vag. & 60 & $6(11.8)$ & $4(7.8)$ \\
\hline Rozet et al. ${ }^{25} 2005$ & 325 & $\mathrm{PE}, 3$ & Only vag. & $14.5^{\mathrm{b}}$ & $8^{c}(2.5)$ & $3(0.9)$ \\
\hline Agarwala et al. ${ }^{26} 2007$ & 72 & $\mathrm{PP}, 1$ & Only vag. & 24 & $1(1.4)$ & 0 \\
\hline Rivoire et al. ${ }^{27} 2007$ & 108 & $\mathrm{PP}, 1$ & Only vag. & $33.7^{b}$ & $9(8.3)$ & $7(6.5)$ \\
\hline Stepanian et al..$^{28} 2008$ & 402 & $\mathrm{PP}, 1$ & $\mathrm{n} / \mathrm{d}$ & 12 & $12(3.0)$ & $5(1.2)$ \\
\hline Deprest et al. ${ }^{29} 2009$ & $104^{\mathrm{d}}$ & $\mathrm{PP}, 1^{\mathrm{d}}$ & Only vag. & $33^{\mathrm{b}}$ & $12(11.5)$ & $8(7.7)^{\mathrm{e}}$ \\
\hline Granese et al. ${ }^{30} 2009$ & 165 & $\mathrm{PP}, 1$ & Yes, both & 43 & $7(4.2)^{\mathrm{f}}$ & $1(0.6)$ \\
\hline Loffeld et al. ${ }^{31} 2009$ & 20 & $\mathrm{PP}, 1$ & Only vag. & $45^{b}$ & $1(5.0)$ & $1(5.0)$ \\
\hline North et al. 322009 & 22 & $\mathrm{PP}, 1$ & Only vag. & $27.5^{\mathrm{b}}$ & $1(4.5)$ & $1(4.5)$ \\
\hline Akladios et al. ${ }^{33} 2010$ & 48 & $\mathrm{PP}, 1$ & Only vag. & $15.8^{\mathrm{b}}$ & $1(2.2)$ & $1(2.2)$ \\
\hline
\end{tabular}


Table 3. Continued

\begin{tabular}{|c|c|c|c|c|c|c|}
\hline First author & $\begin{array}{l}\text { No. of } \\
\text { patients }\end{array}$ & $\begin{array}{l}\text { Material } \\
\text { and type } \\
\text { of mesh }\end{array}$ & $\begin{array}{l}\text { Vaginal/ } \\
\text { rectal } \\
\text { exam }\end{array}$ & $\begin{array}{l}\text { Follow-up } \\
\text { mo } \\
\text { (median) }\end{array}$ & $\begin{array}{l}\text { Mesh } \\
\text { compl. } \\
(\%)\end{array}$ & $\begin{array}{l}\text { Mesh } \\
\text { erosion } \\
(\%)\end{array}$ \\
\hline Sabbagh et al. ${ }^{34} 2010$ & 132 & $\mathrm{PP}, 1$ & Only vag. & 60 & $6(4.5)$ & $5(3.8)$ \\
\hline Maher et al..$^{35} 2011$ & 53 & $\mathrm{PP}, 1$ & Only vag. & $24^{\mathrm{b}}$ & $1(1.9)$ & $1(1.9)$ \\
\hline Sergent et al. ${ }^{36} 2011$ & 116 & PE, 3 & Only vag. & 34.2 & $5(4.3)$ & $4(3.4)$ \\
\hline Perez et al. ${ }^{37} 2011$ & 85 & PE, 3 & Only vag. & 12 & $5(5.9)$ & $3(3.5)$ \\
\hline Price et al. ${ }^{38} 2011$ & 84 & $\mathrm{PP}, 1$ & Only vag. & $24^{b}$ & $5(6.0)$ & $5(6.0)^{g}$ \\
\hline Freeman et al. ${ }^{39} 2013$ & 23 & $\mathrm{PP}, 1$ & Only vag. & 12 & 0 & 0 \\
\hline Freeman et al. ${ }^{39} 2013$ & 23 & $\mathrm{PP}, 1$ & Only vag. & 12 & 0 & 0 \\
\hline Leruth et al. ${ }^{40} 2013$ & 55 & PE, 3 & Only vag. & $25^{b}$ & 0 & 0 \\
\hline Liu et al. ${ }^{41} 2014$ & 39 & $\mathrm{PP}, 1$ & Only vag. & 12 & 0 & 0 \\
\hline Park et al. ${ }^{42} 2014$ & 54 & $\mathrm{PP}, 1$ & Only vag. & $29.7^{b}$ & $3(5.6)$ & $3(5.6)$ \\
\hline Sarlos et al. ${ }^{43} 2014$ & 68 & $\mathrm{PP}, 1$ & Only vag. & $60^{\mathrm{b}}$ & $2(2.9)$ & $2(2.9)$ \\
\hline El Hamamsy and & 220 & $\mathrm{PP}, 1$ & Only vag. & 12 & $2(0.9)$ & $2(0.9)$ \\
\hline \multicolumn{7}{|l|}{ Fayyad $^{44} 2015$} \\
\hline Estrade et al. ${ }^{45} 2015$ & 35 & PE, 3 & Only vag. & 13.2 & $1(2.9)$ & $1(2.9)$ \\
\hline Gracia et al. ${ }^{46} 2015$ & 30 & $\mathrm{PP}, 1$ & Only vag. & 12 & 0 & 0 \\
\hline Vieillefosse et al. ${ }^{47} 2015$ & 100 & $\mathrm{PP}, 1 / \mathrm{PE}, 3$ & Only vag. & 23.6 & $2(2.0)$ & $2(2.0)$ \\
\hline Costatini et al. ${ }^{48} 2016$ & 60 & $\mathrm{PP}, 1$ & Only vag. & $41.7^{\mathrm{b}}$ & $3(5.0)$ & $3(5.0)$ \\
\hline Dandolu et al. ${ }^{3} 2016$ & 4552 & $\mathrm{n} / \mathrm{d}$ & $\mathrm{n} / \mathrm{a}$ & 24 & $52(1.7)$ & $52(1.7)$ \\
\hline Liang et al. ${ }^{49} 2016$ & 30 & $\mathrm{PP}, 1$ & Only vag. & 36 & $3(10)$ & $3(10)$ \\
\hline Lizee et al. ${ }^{50} 2016$ & 60 & PE, 3 & Only vag. & 27 & $1(1.7)$ & $1(1.7)$ \\
\hline \multicolumn{7}{|l|}{2016} \\
\hline Zebede et al. ${ }^{52} 2016$ & 144 & $\mathrm{PP}, 1$ & Only vag. & 21 & $4(2.8)$ & 0 \\
\hline Pan et al..$^{53} 2016$ & 99 & $\mathrm{PP}, 1$ & Only vag. & $33^{b}$ & 0 & 0 \\
\hline Chen and Hua ${ }^{54} 2017$ & 102 & $\mathrm{PP}, 1$ & Only vag. & 24 & $1(1.0)$ & $1(1.0)$ \\
\hline \multicolumn{7}{|l|}{ Robotic } \\
\hline Elliott et al..$^{55} 2007$ & 42 & $\mathrm{PP}, 1$ & Only vag. & $36^{\mathrm{b}}$ & $3(7.1)$ & $2(4.8)$ \\
\hline Benson et al. ${ }^{56} 2010$ & 33 & $\mathrm{PP}, 1$ & $\mathrm{n} / \mathrm{d}$ & $20.7-38.4^{\mathrm{i}}$ & $2(6.1)$ & 0 \\
\hline Shveiky et al..$^{57} 2010$ & 17 & $\mathrm{PP}, 1$ & Only vag. & 12.3 & 0 & 0 \\
\hline Xylinas et al. ${ }^{58} 2010$ & 12 & $P P, 1$ & $\mathrm{n} / \mathrm{d}$ & 19.1 & 0 & 0 \\
\hline Geller et al. ${ }^{59} 2011$ & 15 & $\mathrm{PP}, 1$ & Only vag. & $14.8^{b}$ & $2(13.3)$ & $2(13.3)$ \\
\hline
\end{tabular}


Table 3. Continued

\begin{tabular}{|c|c|c|c|c|c|c|}
\hline First author & $\begin{array}{l}\text { No. of } \\
\text { patients }\end{array}$ & $\begin{array}{l}\text { Material } \\
\text { and type } \\
\text { of mesh }\end{array}$ & $\begin{array}{l}\text { Vaginal/ } \\
\text { rectal } \\
\text { exam }\end{array}$ & $\begin{array}{l}\text { Follow-up } \\
\text { mo } \\
\text { (median) }\end{array}$ & $\begin{array}{l}\text { Mesh } \\
\text { compl. } \\
(\%)\end{array}$ & $\begin{array}{l}\text { Mesh } \\
\text { erosion } \\
(\%)\end{array}$ \\
\hline $\begin{array}{l}\text { Moreno-Sierra et al. }{ }^{60} \\
2011\end{array}$ & 31 & $\mathrm{PP}, 1$ & Only vag. & $24.5^{b}$ & $1(3.2)$ & 0 \\
\hline Shimko et al. ${ }^{61} 2011$ & 40 & $P P, 1$ & Only vag. & 62 & $2(5.0)$ & $2(5.0)$ \\
\hline Geller et al. ${ }^{62} 2012$ & 23 & $\mathrm{PP}, 1$ & Only vag. & $44.2^{\mathrm{b}}$ & $2(8.7)$ & $2(8.7)$ \\
\hline Göçmen et al. ${ }^{63} 2012$ & 12 & $P P, 1$ & $\mathrm{n} / \mathrm{d}$. & 12 & 0 & 0 \\
\hline Mourik et al. ${ }^{64} 2012$ & $50^{j}$ & $\mathrm{PP}, 1$ & Only vag. & 16 & $1(2.0)$ & 0 \\
\hline Siddiqui et al. ${ }^{65} 2012$ & 70 & $\mathrm{PP}, 1$ & Only vag. & $18.3^{\mathrm{b}}$ & $3(4.3)$ & $3(4.3)$ \\
\hline Belsante et al. ${ }^{66} 2013$ & 35 & $\mathrm{PP}, 1$ & Only vag. & 28 & $1(2.9)$ & $1(2.9)$ \\
\hline $\begin{array}{l}\text { Louis-Sylvestre and } \\
\text { Herry } 672013\end{array}$ & 90 & PE, 3 & $\mathrm{n} / \mathrm{d}$ & $15.6^{b}$ & $1(1.1)$ & $1(1.1)$ \\
\hline Salamon et al. ${ }^{68} 2013$ & 118 & $P P, 1$ & Only vag. & 12 & 0 & 0 \\
\hline Barboglio et al. ${ }^{69} 2014$ & 127 & PP, 1 & Only vag. & 12 & $3(2.4)$ & $3(2.4)$ \\
\hline Borahay et al. ${ }^{70} 2014$ & 20 & $\mathrm{PP}, 1$ & Only vag. & $17.3^{\mathrm{b}}$ & 0 & 0 \\
\hline Culligan et al. ${ }^{71} 2014$ & 143 & $\mathrm{PP}, 1$ & Only vag. & 12 & 0 & 0 \\
\hline Ploumidis et al..$^{72} 2014$ & 95 & $\mathrm{PP}, 1$ & Only vag. & 34.8 & $1(1.1)$ & $1(1.1)$ \\
\hline Jambusaria et al. ${ }^{73} 2015$ & 30 & $P P, 1$ & Only vag. & 12 & $1(3.3)$ & $1(3.3)$ \\
\hline Linder et al. ${ }^{74} 2015$ & 70 & $\mathrm{PP}, 1$ & $\mathrm{n} / \mathrm{d}$ & 72 & $2(2.9)$ & $2(2.9)$ \\
\hline Myers et al. ${ }^{75} 2015$ & 83 & $\mathrm{PP}, 1$ & Only vag. & 12.8 & $4(4.8)$ & $4(4.8)$ \\
\hline $\begin{array}{l}\text { Prendergast et al. }{ }^{76} \\
2016\end{array}$ & 33 & $\mathrm{PP}, 1$ & Only vag. & 12 & $2(6.1)$ & $2(6.1)$ \\
\hline Linder et al. ${ }^{77} 2017$ & 132 & $\mathrm{PP}, 1$ & Only vag. & 33 & $8(6.1)$ & $8(6.1)$ \\
\hline \multicolumn{7}{|c|}{ 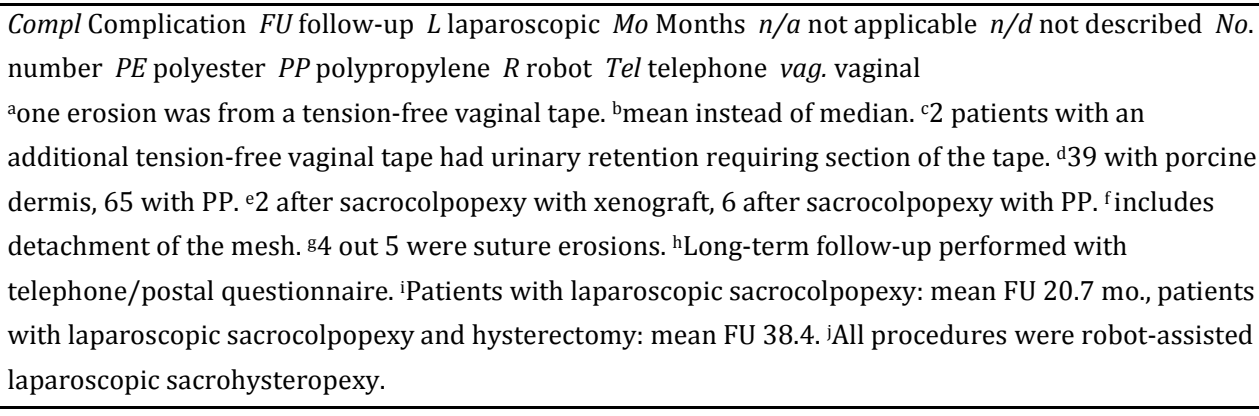 } \\
\hline
\end{tabular}




\section{Discussion}

Synthetic meshes have been used in pelvic reconstructive surgery to reinforce weak or defective supportive tissue since 1959.80 The use of synthetic mesh potentially adds to the complication profile and mesh-related morbidity can have a considerable impact on the quality of life. ${ }^{81}$ The introduction of transvaginal procedures showed a high risk of mesh-related complications. ${ }^{1}$ This study with long-term follow-up shows that meshrelated morbidity following a minimally invasive abdominal pelvic floor repair is low.

In total, there were 3 patients with a mesh erosion (3.1\%), of which 2 were asymptomatic. Two of these 3 patients underwent a concomitant supracervical hysterectomy. A total hysterectomy is associated with a four times higher risk of mesh erosion compared with sacrocolpopexy without hysterectomy. ${ }^{82}$ A subtotal hysterectomy, however, appears to generate mesh erosion rates comparable with patients with a history of a hysterectomy undergoing a sacrocolpopexy. ${ }^{82}$ Other known predictors of mesh erosion include the use of steroids, diabetes, level of surgeon experience, intra-abdominal adhesions and postoperative pelvic hematoma. ${ }^{3,81-84}$

In this study a monofilament and macroporous ( $>75 \mu \mathrm{m}$, type I) mesh was used, allowing host cell colonization with collagen deposition, angiogenesis and infiltration of leukocytes, resulting in good support and a reduced risk of infection. ${ }^{28}$ Research showed that synthetic meshes with smaller pores (type II and III) are associated with a higher erosion rate. ${ }^{81,85}$ It has been suggested that lightweight meshes may be less prone to erosion but may have a higher recurrence rate than heavy-weight grafts. Three studies show a $0 \%$ mesh erosion rate after use of lightweight mesh. ${ }^{41,68,71}$ Studies with longer follow-up or comparative studies for an abdominal prolapse repair, however, do not exist. Data on mesh usage with abdominal hernia repairs suggests an impact of the weight of the mesh, but the optimal balance between weight and porosity is unknown. ${ }^{86}$ No significant difference is observed between synthetic and biological mesh in meshrelated complications. ${ }^{85,87-89}$ Evidence suggests, however, that recurrence rates are higher following a repair with biological mesh compared with synthetic mesh6,29,89,90 To reduce the risk of mesh erosion, we administered preoperative antibiotics, dissected meticulously with strict monitoring of hemostasis to prevent a hematoma, attached the (type I) mesh and closed the incised peritoneum over the mesh. But considering the numerous risk factors and prevention strategies, the occurrence of mesh erosion presumably has a multifactorial origin. Mesh erosion after laparoscopic ventral 
rectopexy has been described to occur in the rectum, vagina or bladder, and strictures or rectovaginal fistulas have also been described. ${ }^{91}$ In this study we have not found rectal mesh erosion, nor did we have patients with symptoms suggesting fistulas or strictures.

Four (4.2\%) patients in this study experienced vaginal discomfort during speculum examination. Two of these 4 patients (both sexually active) occasionally experienced vaginal discomfort in daily life. The possibility of vaginal discomfort, probably due to vaginal atrophy and reduced elasticity of the vaginal wall caused by the mesh, should be considered in the decision to offer pelvic reconstructive surgery using mesh in older sexually active females. Both the rectum and the vagina were examined in this study, but only vaginal erosions were diagnosed. The most probable explanation for this difference is vaginal atrophy, which increases with age. In order to obviate this, surgeons could consider prescribing vaginal estrogen cream pre- and postoperatively.

The erosion rates in the literature are in line with our erosion rates. However, the majority of the studies in the literature were retrospective and lacked a systematic follow-up with a rectal and vaginal examination. Furthermore, this study proves that mesh erosion can also occur asymptomatically. The clinical significance of an asymptomatic mesh erosion is, however, unclear. Only the patient with symptomatic mesh erosion underwent surgical intervention in our series. Because of the difference in methods and follow-up, the retrospective design and the lack of mentioning asymptomatic erosions, it is likely that erosion rates are underestimated in the current literature. We believe that the erosion rate in this study approaches the true rate.

The strong points of this study were its prospective nature, with the use of validated questionnaires and standardized follow-up examinations to confirm our findings. Loss to follow-up was low considering the long duration of the study and reasons for loss to follow-up were known. Furthermore, solely type 1 mesh was used throughout this study, minimizing heterogeneity and variability. Another strong point is that it reports not only on sacrocolpopexy, but also on combined sacrocolporectopexy, making the results more widely applicable.

The most important limitation of this study is that all patients were treated in a single tertiary referral hospital for pelvic floor disorders. Some of the patients had complex pelvic floor disorders and/or an extensive history of pelvic floor surgery, therefore limiting the generalizability of the results. In addition, $26.1 \%$ of all invited patients were 
not physical examined for various reasons, and therefore bias may have occurred. These patients were, however, assessed using a questionnaire specifically assessing erosion related complaints. We aimed for a 5-year follow-up, however, the follow-up time ended up being 48.1 months. Most patients were examined between 48 and 60 months, therefore limiting our Kaplan Meier estimates at the exact time point of 60 months. We added 95\% confidence intervals to make our results more accurate and interpretable with the wider range of follow-up. Results of the Kaplan-Meier curve should therefore be interpret with caution. Another limitation is that we did not perform a power-analysis. This study was set up as an observational cohort study, and our hypothesis, based on literature, was to find a low incidence and significant prognostic factors were therefore not expected. In our literature review, studies with different inclusion criteria and methods were included. This impaired the homogeneity of the literature results.

Mesh-related morbidity is an important issue because of the potential impact on the quality of life, the widespread use of mesh and the global attention to the topic. In recent years the public opinion has turned fiercely against the use of synthetic grafts. Fear of mesh-related morbidity is resulting in under-treatment of all serious, disabling pelvic floor disorders. The results of this study and the literature review demonstrate that abdominally placed synthetic meshes for pelvic reconstructive surgery has a low complication rate in the long-term. This is an encouraging finding for patients, doctors and governmental institutions, in a field marked by a lack of knowledge about the use of mesh. Surgeons using synthetic mesh for pelvic floor repair are encouraged to perform focused and meticulous examinations looking for mesh erosion in the long-term to confirm these results. 


\section{References}

1. US Food and Drug Administration. FDA safety communication: Urogynecologic surgical mesh: update on the safety and effectiveness of transvaginal lacement for pelvic organ prolapse. Rev lit arts am. Available at: http://www.fda.gov/downloads/medical. 2011; Accessed 28 November 2016.

2. Abed H, Rahn DD, Lowenstein L, Balk EM, Clemons JL, Rogers RG, for the systematic review group of the Society of Gynecologic Surgeons. Incidence and management of graft erosion, wound granulation, and dyspareunia following vaginal prolapse repair with graft materials: a systematic review. Int Urogynecol J. 2011; 22:789-798.

3. Dandolu V, Akiyama M, Allenback G, Pathak P. Mesh complications and failure rates after transvaginal mesh repair compared with abdominal or laparoscopic sacrocolpopexy and to native tissue repair in treating apical prolapse. Int Urogynecol J. 2016;28:215-222.

4. Serati M, Bogani G, Sorice P, Braga A, Torella M, Salvatore S, et al. Robot-assisted sacrocolpopexy for pelvic organ prolapse: a systematic review and meta-analysis of comparative studies. Eur Urol. 2014;66:303-318.

5. Paraiso MFR, Jelovsek JE, Frick A, Chen CCG, Barber MD. Laparoscopic compared with robotic sacrocolpopexy for vaginal prolapse: a randomized controlled trial. Obstet Gynecol. 2011;118:1005-1013.

6. van Iersel JJ, Paulides TJC, Verheijen PM, Lumley JW, Broeders IAMJ, Consten ECJ. Current status of laparoscopic and robotic ventral mesh rectopexy for external and internal rectal prolapse. World J Gastroenterol 2016;22:4977-4987.

7. van Iersel JJ, de Witte CJ, Verheijen PM, Broeders IAMJ, Lenters E, Consten ECJ, et al. Robot-assisted sacrocolporectopexy for multicompartment prolapse of the pelvic floor: a prospective cohort study evaluating functional and sexual outcome. Dis Colon Rectum. 2016;59:968-974.

8. Unger CA, Paraiso MFR, Jelovsek JE, Barber MD, Ridgeway B. Perioperative adverse events after minimally invasive abdominal sacrocolpopexy. Am J Obstet Gynecol. 2014;211:547.e1-8.

9. Wijffels NA, Collinson R, Cunningham C, Lindsey I. What is the natural history of internal rectal prolapse? Colorectal Dis. 2010;12:822-830.

10. Swift S, Morris S, McKinnie V, Freeman R, Petri E, Scotti RJ, et al. Validation of a simplified technique for using the POPQ pelvic organ prolapse classification system. Int Urogynecol J Pelvic Floor Dysfunct. 2006;17:615-620.

11. Haylen BT, Freeman RM, Swift SE, Cosson M, Davila GW, Deprest J, et al. An International Urogynecological Association (IUGA)/International Continence Society (ICS) joint terminology and classification of the complications related directly to the insertion of prostheses (meshes, implants, tapes) and grafts in female pelvic floor surgery. Int Urogynecol J. 2011;22:3-15.

12. Utomo E, Korfage IJ, Wildhagen MF, Steensma AB, Bangma CH, Blok BFM. Validation of the urogenital distress inventory (UDI-6) and incontinence impact questionnaire (IIQ-7) in a Dutch population. Neurourol Urodyn. 2015; 34:24-31.

13. Utomo E, Blok BF, Steensma AB, Korfage IJ. Validation of the pelvic floor distress inventory (PFDI20) and pelvic floor impact questionnaire (PFIQ-7) in a Dutch population. Int Urogynecol J. 2014;25:531-544. 
14. Clifton MM, Pizarro-Berdichevsky J, Goldman HB.Robotic female pelvic floor reconstruction: a review. Urology. 2016;91:33-40.

15. Chan SSC, Pang SMW, Cheung TH, Cheung RYK, Chung TKH. Laparoscopic sacrocolpopexy for the treatment of vaginal vault prolapse: with or without robotic assistance. Hong Kong Med J. 2011;17:54-60.

16. Tan-Kim J, Menefee SA, Luber KM, Nager CW, Lukacz ES. Robotic-assisted and laparoscopic sacrocolpopexy: comparing operative times, costs and outcomes. Female Pelvic Med Reconstr Surg. 2011;17:44-49.

17. Seror J, Yates DR, Seringe E, Vaessen C, Bitker M-O, Chartier-Kastler E, et al. Prospective comparison of short-term functional outcomes obtained after pure laparoscopic and robotassisted laparoscopic sacrocolpopexy. World J Urol. 2013;30:393-398.

18. Joubert M, Thubert T, Lefranc J-P, Vaessen C, Chartier-Kastler E, Deffieux X, Rouprêt M. Comparison of functional outcomes with purely laparoscopic sacrocolpopexy and robot-assisted sacrocolpopexy in obese women. Prog Urol. 2014;24:1106-1113.

19. Tan-Kim J, Nager CW, Grimes CL, Luber KM, Lukacz ES, Brown HW, et al. A randomized trial of vaginal mesh attachment techniques for minimally invasive sacrocolpopexy. Int Urogynecol J. 2015;26:649-656.

20. Kenton K, Mueller ER, Tarney C, Bresee C, Anger JT. One-year outcomes after minimally invasive sacrocolpopexy. Female Pelvic Med Reconstr Surg. 2016;22:382-384.

21. Antiphon P, Elard S, Benyoussef A, Fofana M, Yiou R, Gettman M, et al. Laparoscopic promontory sacral colpopexy: is the posterior, recto-vaginal, mesh mandatory? Eur Urol. 2004;45:655-661.

22. Gadonneix P, Ercoli A, Salet-Lizée D, Cotelle O, Bolner B, Van Den Akker M, et al. Laparoscopic sacrocolpopexy with two separate meshes along the anterior and posterior vaginal walls for multicompartment pelvic organ prolapse. J Am Assoc Gynecol Laparosc. 2004;11:29-35.

23. Paraiso MFR, Walters MD, Rackley RR, Melek S, Hugney C. Laparoscopic and abdominal sacral colpopexies: a comparative cohort study. Am J Obstet Gynecol. 2005;192:1752-1758.

24. Ross JW, Preston M. Laparoscopic sacrocolpopexy for severe vaginal vault prolapse: five-year outcome. J Minim Invasive Gynecol. 2005;12:221-226.

25. Rozet F, Mandron E, Arroyo C, Andrews H, Cathelineau X, Mombet A, et al. Laparoscopic sacral colpopexy approach for genito-urinary prolapse: experience with 363 cases. Eur Urol. 2005;47:230-236.

26. Agarwala N, Hasiak N, Shade M. Laparoscopic sacral colpopexy with Gynemesh as graft materialexperience and results. J Minim Invasive Gynecol. 2007;14:577-583.

27. Rivoire C, Botchorishvili R, Canis M, Jardon M, Rabischong B, Wattiez A, et al. Complete laparoscopic treatment of genital prolapse with meshes including vaginal promontofixation and anterior repair: a series of 138 patients. J Minim Invasive Gynecol. 2007;14:712-718.

28. Stepanian AA, Miklos JR, Moore RD, Mattox TF. Risk of mesh extrusion and other mesh-related complications after laparoscopic sacral colpopexy with or without concurrent laparoscopicassisted vaginal hysterectomy: experience of 402 patients. J Minim Invasive Gynecol. 2008;15:188196.

29. Deprest J, De Ridder D, Roovers J-P, Werbrouck E, Coremans G, Claerhout F. Medium term outcome of laparoscopic sacrocolpopexy with xenografts compared to synthetic grafts. J Urol. 2009;182:2362-2368. 
30. Granese R, Candiani M, Perino A, Romano F, Cucinella G. Laparoscopic sacrocolpopexy in the treatment of vaginal vault prolapse: 8 years experience. Eur J Obstet Gynecol Reprod Biol. 2009;146:227-231.

31. Loffeld CJW, Thijs S, Mol BW, Bongers MY, Roovers J-PWR. Laparoscopic sacrocolpopexy: a comparison of Prolene and Tutoplast mesh. Acta Obstet Gynecol Scand. 2009;88:826-830.

32. North CE, Ali-Ross NS, Smith ARB, Reid FM. A prospective study of laparoscopic sacrocolpopexy for the management of pelvic organ prolapse. BJOG. 2009;116:1251-1257.

33. Akladios CY, Dautun D, Saussine C, Baldauf JJ, Mathelin C, Wattiez A. Laparoscopic sacrocolpopexy for female genital organ prolapse: establishment of a learning curve. Eur J Obstet Gynecol Reprod Biol. 2010;149:218-221.

34. Sabbagh R, Mandron E, Piussan J, Brychaert PE, Tu LM. Long-term anatomical and functional results of laparoscopic promontofixation for pelvic organ prolapse. BJU Int. 2010;106:861-866.

35. Maher CF, Feiner B, DeCuyper EM, Nichlos CJ, Hickey KV, O’Rourke P. Laparoscopic sacral colpopexy versus total vaginal mesh for vaginal vault prolapse: a randomized trial. Am J Obstet Gynecol. 2011;204:360.e1-7.

36. Sergent F, Resch B, Loisel C, Bisson V, Schaal J-P, Marpeau L. Mid-term outcome of laparoscopic sacrocolpopexy with anterior and posterior polyester mesh for treatment of genito-urinary prolapse. Eur J Obstet Gynecol Reprod Biol. 2011;156:217-222.

37. Perez T, Crochet P, Descargues G, Tribondeau P, Soffray F, Gadonneix P, et al. Laparoscopic sacrocolpopexy for management of pelvic organ prolapse enhances quality of life at one year: a prospective observational study. J Minim Invasive Gynecol. 2011;18:747-754.

38. Price N, Slack A, Jackson SR. Laparoscopic sacrocolpopexy: an observational study of functional and anatomical outcomes. Int Urogynecol J. 2011;22;77-82.

39. Freeman RM, Pantazis K, Thomson A, Frappell J, Bombieri L, Moran P, et al. A randomised controlled trial of abdominal versus laparoscopic sacrocolpopexy for the treatment of posthysterectomy vaginal vault prolapse: LAS study. Int Urogynecol J. 2013;24:377-384.

40. Leruth J, Fillet M, Waltregny D. Incidence and risk factors of postoperative stress urinary incontinence following laparoscopic sacrocolpopexy in patients with negative preoperative prolapse reduction stress testing. Int Urogynecol J. 2013;24:485-491.

41. Liu C-K, Tsai C-P, Chou M-M, Shen PS, Chen GD, Hung YC, et al. A comparative study of laparoscopic sacrocolpopexy and total vaginal mesh procedure using lightweight polypropylene meshes for prolapse repair. Taiwan J Obstet Gynecol. 2014;53:552-558.

42. Park YH, Yang SC, Park ST, Park SH, Kim HB. Laparoscopic reconstructive surgery is superior to vaginal reconstruction in the pelvic organ prolapse. Int J Med Sci. 2014;11:1082-1088.

43. Sarlos D, Kots L, Ryu G, Schaer G. Long-term follow-up of laparoscopic sacrocolpopexy. Int Urogynecol J. 2014;25:1207-1212.

44. El Hamamsy D, Fayyad AM. New onset stress urinary incontinence following laparoscopic sacrocolpopexy and its relation to anatomical outcomes. Int Urogynecol J. 2015;26:1041-1045.

45. Estrade J-P, Gurriet B, Franquebalme J-P, Chinchole J-M, Glowaczower E, Ferry C, et al. Laparoscopic sacrocolpopexy with a vaginal prosthetic adhesive. Gynecol Obstet Fertil. 2015;43:419-423. 
46. Gracia M, Perello M, Bataller E, Espuna M, Parellada M, Genis D, et al. Comparison between laparoscopic sacral hysteropexy and subtotal hysterectomy plus cervicopexy in pelvic organ prolapse: a pilot study. Neurourol Urodyn. 2015;34:654-658.

47. Vieillefosse S, Thubert T, Dache A, Hermieu J-F, Deffieux X. Satisfaction, quality of life and lumbar pain following laparoscopic sacrocolpopexy: suture vs. tackers. Eur J Obstet Gynecol Reprod Biol. 2015;187:51-56.

48. Costantini E, Mearini L, Lazzeri M, Bini V, Nunzi E, di Biase M, et al. Laparoscopic versus abdominal sacrocolpopexy: a randomized, controlled trial. J Urol. 2016;196:159-165.

49. Liang S, Zhu L, Song X, Xu T, Sun Z, Lang J. Long-term outcomes of modified laparoscopic sacrocolpopexy for advanced pelvic organ prolapse: a 3-year prospective study. Menopause. 2016;23:765-770.

50. Lizee D, Campagna G, Morciano A, Panico G, Ercoli A, Gadonneix P. Laparoscopic sacral colpopexy: how to place the posterior mesh into rectovaginal space? Neurourol Urodyn. 2016;9999:1-6

51. Vandendriessche D, Sussfeld J, Giraudet G, Lucot J-P, Behal H, Cosson M. Complications and reoperations after laparoscopic sacrocolpopexy with a mean follow-up of 4 years. Int Urogynecol J. 2016;28:231-239.

52. Zebede S, Dawood A, Alarab M, Drutz H, Lovatsis D. A streamlined surgical approach to laparoscopic sacrocolpopexy for post-hysterectomy vault prolapse. J Obstet Gynaecol Can. 2016;38:446-452.

53. Pan K, Cao L, Ryan NA, Wang Y, Xu H. Laparoscopic sacral hysteropexy versus laparoscopic sacrocolpopexy with hysterectomy for pelvic organ prolapse. Int Urogynecol J. 2016;27:93-101.

54. Chen Y, Hua K. Medium-term outcomes of laparoscopic sacrocolpopexy or sacrohysteropexy versus vaginal sacrospinous ligament fixation for middle compartment prolapse. Int J Gynecol Obstet. 2017;137:164-169.

55. Elliott DS, Siddiqui SA, Chow GK. Assessment of the durability of robot-assisted laparoscopic sacrocolpopexy for treatment of vaginal vault prolapse. J Robot Surg. 2007;1:163-168.

56. Benson AD, Kramer BA, Wayment RO, Schwartz BF. Supracervical robotic-assisted laparoscopic sacrocolpopexy for pelvic organ prolapse. JSLS. 2010;14:525-530.

57. Shveiky D, Iglesia CB, Sokol AI, Kudish BI, Gutman RE. Robotic sacrocolpopexy versus vaginal colpopexy with mesh: choosing the right surgery for anterior and apical prolapse. Female Pelvic Med Reconstr Surg. 2010;16:121-127.

58. Xylinas E, Ouzaid I, Durand X, Ploussard G, Salomon L, Gillion N, et al. Robot-assisted laparoscopic sacral colpopexy: initial experience in a high-volume laparoscopic reference center. J Endourol. 2010;24:1985-1989.

59. Geller EJ, Parnell BA, Dunivan GC. Pelvic floor function before and after robotic sacrocolpopexy: one-year outcomes. J Minim Invasive Gynecol. 2011;18:322-327.

60. Moreno Sierra J, Ortiz Oshiro E, Fernandez Pérez C, Galante Romo I, Corral Rosillo J, Prieto Nogal S, et al. Long-term outcomes after robotic sacrocolpopexy in pelvic organ prolapse: prospective analysis. Urol Int. 2011;86:414-418.

61. Shimko MS, Umbreit EC, Chow GK, Elliott DS. Long-term outcomes of robotic-assisted laparoscopic sacrocolpopexy with a minimum of three years follow-up. J Robot Surg. 2011;5:175-180.

62. Geller EJ, Parnell BA, Dunivan GC. Robotic vs abdominal sacrocolpopexy: 44-month pelvic floor outcomes. Urology. 2012;79:532-536. 
63. Göçmen A, Sanlıkan F, Uçar MG. Robotic-assisted sacrocolpopexy/sacrocervicopexy repair of pelvic organ prolapse: initial experience. Arch Gynecol Obstet. 2012;285:683-688.

64. Mourik SL, Martens JE, Aktas M. Uterine preservation in pelvic organ prolapse using robot assisted laparoscopic sacrohysteropexy: quality of life and technique. Eur J Obstet Gynecol Reprod Biol. 2012;165:122-127.

65. Siddiqui NY, Geller EJ, Visco AG. Symptomatic and anatomic 1-year outcomes after robotic and abdominal sacrocolpopexy. Am J Obstet Gynecol. 2012;206:435.e1-5.

66. Belsante M, Murray S, Dillon B, Zimmern P. Mid term outcome of robotic mesh sacrocolpopexy. Can J Urol. 2013;20:6656-6661.

67. Louis-Sylvestre C, Herry M. Robotic-assisted laparoscopic sacrocolpopexy for stage III pelvic organ prolapse. Int Urogynecol J. 2013;24:731-733.

68. Salamon CG, Lewis C, Priestley J, Gurshumov E, Culligan PJ. Prospective study of an ultralightweight polypropylene Y mesh for robotic sacrocolpopexy. Int Urogynecol J. 2013;24:13711375.

69. Barboglio PG, Toler AJW, Triaca V. Robotic sacrocolpopexy for the management of pelvic organ prolapse: a review of midterm surgical and quality of life outcomes. Female Pelvic Med Reconstr Surg. 2014;20:38-43.

70. Borahay MA, Oge T, Walsh TM, Patel PR, Rodriguez AM, Kilic GS. Outcomes of robotic sacrocolpopexy using barbed delayed absorbable sutures. J Minim Invasive Gynecol. 2014;21:412416.

71. Culligan PJ, Gurshumov E, Lewis C, Priestley JL, Komar J, Shah N, et al. Subjective and objective results 1 year after robotic sacrocolpopexy using a lightweight Y-mesh. Int Urogynecol J. 2014;25:731-735.

72. Ploumidis A, Spinoit A-F, De Naeyer G, Schatteman P, Gan M, Ficarra V, et al. Robot-assisted sacrocolpopexy for pelvic organ prolapse: surgical technique and outcomes at a single high-volume institution. Eur Urol. 2014;65:138-145.

73. Jambusaria LH, Murphy M, Lucente VR. One-year functional and anatomic outcomes of robotic sacrocolpopexy versus vaginal extraperitoneal colpopexy with mesh. Female Pelvic Med Reconstr Surg. 2015;21:87-92.

74. Linder BJ, Chow GK, Elliott DS. Long-term quality of life outcomes and retreatment rates after robotic sacrocolpopexy. Int J Urol. 2015;22:1155-1158.

75. Myers EM, Siff L, Osmundsen B, Geller E, Matthews CA. Differences in recurrent prolapse at 1 year after total vs supracervical hysterectomy and robotic sacrocolpopexy. Int Urogynecol J. 2015;26:585-589.

76. Prendergast E, Silver H, Johnson LL, Simon M, Feinglass J, Kielb S, et al. Anatomic outcomes of robotic assisted supracervical hysterectomy and concurrent sacrocolpopexy at a tertiary care institution at initial adaptation of the procedure. Female Pelvic Med Reconstr Surg. 2016;22:29-32.

77. Linder BJ, Anand M, Klingele CJ, Trabuco EC, Gebhart JB, Occhino JA. Outcomes of robotic sacrocolpopexy using only absorbable suture for mesh fixation. Female Pelvic Med Reconstr Surg. 2017;23:13-16.

78. Sullivan ES, Longaker CJ, Lee PY. Total pelvic mesh repair: a ten-year experience. Dis Colon Rectum. 2001;44:857-863. 
79. Lim M, Sagar PM, Gonsalves S, Thekkinkattil D, Landon C. Surgical management of pelvic organ prolapse in females: functional outcome of mesh sacrocolpopexy and rectopexy as a combined procedure. Dis Colon Rectum. 2007;50:1412-1421.

80. Wu JS.Rectal prolapse: a historical perspective. Curr Probl Surg. 2009;46:602-716.

81. Bako A, Dhar R. Review of synthetic mesh-related complications in pelvic floor reconstructive surgery. Int Urogynecol J. 2009;20:103-111.

82. Gutman R, Maher. Uterine-preserving POP surgery. Int Urogynecol J. 2013;24:1803-13.

83. Trilling B, Martin G, Faucheron J-L. Mesh erosion after laparoscopic rectopexy: a benign complication? Color Dis. 2014;16:832-833.

84. Deffieux X, Letouzey V, Savary D, Sentilhes L, Agostini A, Mares P, et al. Prevention of complications related to the use of prosthetic meshes in prolapse surgery: guidelines for clinical practice. Eur J Obstet Gynecol Reprod Biol. 2012;165:170-180.

85. Evans C, Stevenson AR, Sileri P, Mercer-Jones MA, Dixon AR, Cunningham C, et al. A multicenter collaboration to assess the safety of laparoscopic ventral rectopexy. Dis Colon Rectum. 2015;58:799-807.

86. Earle DB, Mark LA.Prosthetic material in inguinal hernia repair: how do I choose? Surg Clin North Am. 2008;88:179-201.

87. Smart NJ, Pathak S, Boorman P, Daniels IR.Synthetic or biological mesh use in laparoscopic ventral mesh rectopexy-a systematic review. Color Dis. 2013;15:650-654.

88. Ogilvie JW, Stevenson ARL, Powar M. Case-matched series of a non-cross-linked biologic versus non-absorbable mesh in laparoscopic ventral rectopexy. Int J Colorectal Dis. 2014;29:1477-1483.

89. Tate SB, Blackwell L, Lorenz DJ, Steptoe MM, Culligan PJ. Randomized trial of fascia lata and polypropylene mesh for abdominal sacrocolpopexy: 5-year follow-up. Int Urogynecol J. 2011;22:137-143.

90. Culligan PJ, Blackwell L, Goldsmith LJ, Graham CA, Rogers A, Heit MH. A randomized controlled trial comparing fascia lata and synthetic mesh for sacral colpopexy. Obstet Gynecol. 2005;106:2937.

91. Badrek-Amoudi AH, Roe T, Mabey K, Carter H, Mills A, Dixon AR. Laparoscopic ventral mesh rectopexy in the management of solitary rectal ulcer syndrome: a cause for optimism? Colorectal Dis. 2013;15:575-581. 


\section{Appendix A. An overview of the literature}

A computerized search in Pubmed/Medline for English-language articles until January 31th, 2017 was performed, using the terms: 'sacrocolpopex*' OR 'sacral colpopex*' OR 'sacrocervicopex*' OR 'cervicopex*' OR 'colpopex*' OR '(rectopex* AND sacr*)' OR '(rectopex* AND colp*)' OR 'sacrocolporectopex*' OR 'colporectopex' ${ }^{*}$ OR 'rectovaginopex*' OR 'sacrocolpoperineopex*' OR 'colpoperineopex*'. Studies describing mesh erosion after minimal invasive sacrocolpopexy with follow-up of $\geq 12$ months were included (Figure 3). Titles and abstracts were scrutinized by 2 researchers independently (FvZ,JvI). Full manuscripts of all citations that were likely to meet the predefined selection criteria were obtained. References were scanned to identify other potentially eligible articles. Articles describing solely non-synthetic types of mesh and pediatric articles were excluded. Studies describing mesh erosion after sacrocolporectopexy were described separately. Both open and minimal invasive procedures were included, as the number of studies describing mesh erosion after the combined approach were limited. No separate distinction in follow-up duration was made.

Figure 3. Appendix A Flowchart of included studies.

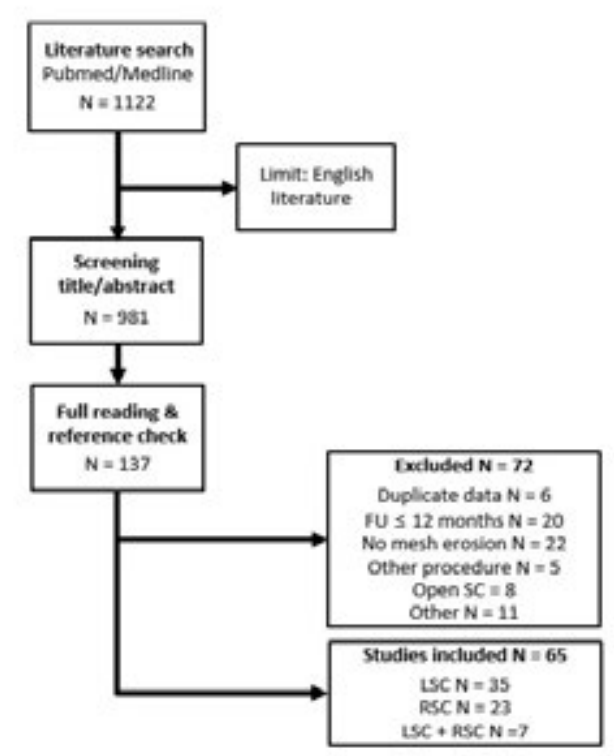

$L S C$ laparoscopic sacrocolpopexy $R S C$ robot-assisted laparoscopic sacrocolpopexy $S C$ sacrocolpopexy 




\section{Chapter 9}

\section{Slechte sinaasappelnetjes, of is er meer onder de zon?}

Steven E. Schraffordt Koops, Femke van Zanten, Egbert Lenters, Esther C.J. Consten, Paul M. Verheijen, Ivo A.M.J. Broeders 


\section{Introductie}

Genitale prolaps is een veel voorkomende benigne gynaecologische aandoening. Het betreft het uitzakken van de vaginawanden of de uterus buiten de normale anatomische positie. Deze aandoening komt na een zwangerschap bij circa 50\% van de vrouwen voor en wordt veroorzaakt door zwakte van de m. levator ani, de fascia rectovaginalis en het ligamentum sacrouterina. ${ }^{1}$ Deze weliswaar benigne aandoening geeft veel klachten en een forse vermindering van kwaliteit van leven voor aangedane vrouwen. Ongeveer 11$19 \%$ van alle vrouwen ondergaat eens in haar leven een chirurgische correctie vanwege prolaps klachten of urine-incontinentie. ${ }^{2} \mathrm{Na}$ een primaire, traditionele chirurgische correctie van vaginale prolaps is bij $30 \%$ van hen een her-operatie noodzakelijk in verband met een recidief prolaps. ${ }^{3}$ Vanwege dit aanzienlijke recidiefpercentage zijn er ruim tien jaar geleden verschillende typen synthetische implantaten geïntroduceerd voor transvaginale chirurgie. Deze 'mesh kits' werden gebruikt om de anatomische positie van de vagina te herstellen en de vaginawanden te verstevigen ter behandeling van prolaps en voorkoming van een recidief. Hetzelfde materiaal wordt al veel langer gebruikt bij tal van ingrepen, waaronder lies- en buikwandbreuken, abdominale prolaps chirurgie voor zowel gynaecologische als darm verzakking en anti urine-incontinentie operaties. Hoewel de resultaten en complicaties van deze operaties van elkaar verschillen, worden ze vaak door elkaar genoemd en beoordeeld.

Al enkele jaren is er veel ophef over het gebruik van deze synthetische materialen bij vaginale chirurgie, omdat dit geassocieerd blijkt te zijn met verschillende ernstige complicaties. In juni 2011 publiceerde de 'U.S. Food and Drug Administration' (FDA) een waarschuwing voor het plaatsen van vaginale implantaten in verband met deze complicaties. ${ }^{4}$ De beschreven complicaties voor vaginale implantaten zijn; het zichtbaar worden van de mesh door de vaginawand (mesh exposure), blaas of rectum (mesh erosion), infectie, bloedingen, pijn, dyspareunie, urinewegproblematiek, incontinentie en recidief prolaps. ${ }^{5}$

Hierover publiceerde de Volkskrant een artikel in november 2011 genaamd 'Schandaal in de bekkenbodem'. In dit artikel uitten patiënten en professionals hun onvrede, problemen, waarschuwingen en twijfels omtrent de vaginale implantaten, ook wel 'matjes' of 'mesh' genoemd. In 2016 heeft de FDA deze transvaginale implantaten als klasse 3, ofwel hoog risicovol genoemd. De FDA heeft op 16 april 2019 de laatste fabrikanten, Boston Scientific en Coloplast, verboden hun mesh te verkopen voor deze specifieke operatietechniek van transvaginale mesh-implantatie. ${ }^{6}$ 
Het onderwerp is ook diverse malen in nationale en internationale televisieprogramma's aan bod gekomen. Gebruik van implantaten zou vrouwen met prolaps meer problemen dan oplossingen opleveren. Kunnen zij deze procedures beter weigeren? Of geen operatie ondergaan? In een van de televisieprogramma's werd een vergelijking met een sinaasappelnetje gemaakt. De schrik voor 'sinaasappel' matjes zit er sindsdien in ieder geval goed in.

Implantaten worden in de geneeskunde al meer dan 50 jaar gebruikt. De vraag is of deze zeer negatieve beoordeling van transvaginale implantaten ook voor alle andere vormen van gebruik van mesh zulke negatieve gevolgen voor patiënten hebben. De term implantaat, matje of mesh heeft een dusdanig negatieve impact gekregen dat patiënten hierdoor niet meer naar een dokter durven te gaan voor een verzakking. Ook andere chirurgische ingrepen met niet lichaamseigen materiaal staan nu ter discussie. Aan de hand van de huidige literatuur lijken implantaat-gerelateerde problemen vooral gerelateerd aan transvaginale mesh implantatie, waarbij eerder de operatieve techniek in combinatie met het gebruik van een implantaat dan het kunststof materiaal zelf de complicaties veroorzaakt.

\section{Effectiviteit plaatsingstechnieken vergeleken}

Genitale prolaps kan zowel via de vaginale als via de abdominale benadering behandeld worden. Bij de behandeling van prolaps van de vaginatop, heeft de abdominale sacrocolpopexie (ASC) de voorkeur, omdat met deze ingreep minder recidieven worden gezien. ${ }^{1}$ Patiënt tevredenheid werd behaald in $84-100 \% .{ }^{7}$ Deze operatie werd al in de jaren vijftig van de vorige eeuw beschreven in de medische literatuur door de gynaecoloog Lane. ${ }^{8}$ De ingreep heeft door de jaren heen steeds modificaties ondergaan, onder meer door de voortschrijdende techniek. Zo wordt deze ingreep nu meestal laparoscopisch of met behulp van de robot verricht. ${ }^{9}$

Om de anatomie van de vagina te herstellen wordt bij deze procedure een synthetisch implantaat enerzijds op de vaginavoorwand, -achterwand en -top en anderzijds aan het ligamentum longitudinale van het sacrum gehecht. ${ }^{9}$ Bij rectum prolaps is de laparoscopische ventrale rectopexie (LVR) in grote delen van Europa en in Nederland de gouden standaard. Dit is een relatief nieuwe techniek, waarbij het implantaat abdominaal op de ventrale zijde van het distale rectum wordt geplaatst en proximaal aan het ligamentum longitudinale ter plaatse van het promontorium wordt gehecht. ${ }^{10} \mathrm{De}$ twee ingrepen kunnen ook gecombineerd worden uitgevoerd. 
Bij vergelijkend gerandomiseerd onderzoek naar de effectiviteit van implantaten middels transvaginale mesh prolaps chirurgie en ASC, blijkt de abdominale benadering significant betere resultaten te geven met succespercentages van $77 \%$ na 6 maanden, versus $44 \%$ na transvaginale mesh chirurgie $(p<0.001)$. De patiënt tevredenheid in de ASC groep was tevens significant hoger. ${ }^{11}$ Ook andere traditionele vaginale benaderingen van gynaecologische prolaps zoals Manchester-Fothergill, vaginale plastieken en sacro-uterine fixatie lijken niet het langdurige succespercentage te halen als de abdominale benadering met gebruik van implantaat, met name niet bij patiënten met recidiverende prolaps. ${ }^{1,12,13}$

Bij de behandeling van rectum prolaps lijkt een abdominaal geplaatst implantaat eveneens zeer effectief te zijn. Lange termijn resultaten tonen een recidief percentage van $8.2 \%$ na 10 jaar (95\% CI 3.7-12.7). Daarnaast werd tevens een sterke afname van fecale incontinentie en obstipatie beschreven. ${ }^{10}$

Naar aanleiding van deze gegevens kan geconcludeerd worden dat abdominale implantaat gebruik zeker effectief is ter behandeling van prolaps. In de publiciteit wordt echter geen aandacht aan de plaatsingstechniek van de implantaten besteed. Dit blijkt echter wel van groot belang te zijn. De FDA meldde destijds (2011) al dat abdominaal geplaatste implantaten minder vaak complicaties veroorzaken dan transvaginaal geplaatste implantaten. ${ }^{4}$ De FDA waarschuwde daarom met name voor de plaatsing van 'transvaginale implantaten'.

\section{Complicaties vergeleken}

Dat complicaties vaker voorkomen na een transvaginale dan na een abdominale procedure blijkt uit vergelijkend onderzoek naar complicatie percentages van vaginale en abdominale implantaten. De gerandomiseerde studie van Maher rapporteerde dat $2 \%$ van de vrouwen na abdominaal gebruik van mesh in vergelijking met $13 \%$ van de vrouwen na een plaatsing van vaginale mesh, een expositie ontwikkelt. Vijf procent van de vrouwen in de abdominale groep had een tweede chirurgische ingreep nodig in verband met recidief of complicaties tegen $22 \%$ in de vaginale groep. ${ }^{11}$ Een heroperatie in verband met complicaties of recidief was vier keer zo vaak nodig in de vaginale groep in vergelijking met de abdominale groep. Systematische reviews die uitkomsten na robot-geassisteerde, laparoscopische en open ASC beschrijven, toonden respectievelijk de volgende gemiddelde mesh complicaties: $2 \%(\mathrm{~N}=1417), 3 \%(\mathrm{~N}=1221)$ en $3.4 \%$ $(\mathrm{N}=2178)^{7,14,15} \mathrm{Na}$ vaginale implantatie van mesh werd in een vergelijkbaar review artikel een percentage van $10,3 \%$ gerapporteerd $(\mathrm{N}=11785){ }^{4}$ 
Amerikaans onderzoek naar abdominaal geplaatste implantaten bij de sacrocolpopexie liet een hogere erosie kans zien met name na langere follow-up. Dit onderzoek wordt vaak aangehaald om de hoge kansen op complicaties aan te tonen. In dit onderzoek werd gebruik gemaakt van Goretex (7\%), mersilene (43\%) en biologisch materiaal $(17 \%)$ voor de suspensie van de vaginatop. ${ }^{16}$ Deze materialen worden in Nederland al jaren bijna niet meer gebruikt vanwege hun hoge recidief kans (biologisch materiaal) of de hogere kans op postoperatieve complicaties (Mersilene en Goretex). In zeer recent gepubliceerd fraai onderzoek met lange follow-up (86 maanden, inclusie vanaf 1996) werden 331 patiënten na laparoscopische ASC geïncludeerd. Een heroperatie in verband met mesh-gerelateerde complicaties werd beschreven in 19 van de 270 onderzochte patiënten (7.0\%). ${ }^{13}$ Bij 83\% van de patiënten werd een (sterke) verbetering van de klachten beschreven. Mogelijk nemen mesh-gerelateerde complicaties dus toe in de tijd. De studie gebruikte door de jaren een steeds lichter gewicht Polypropyleen mesh om te trachten de implantaat gerelateerde complicaties te verlagen. Het is hierbij nog niet duidelijk of dit tot een hoger recidief percentage kan leiden.

In ons centrum werd prospectief onderzoek verricht naar de effectiviteit van het gebruik van de da Vinci operatie robot voor het verrichtten van abdominale prolaps chirurgie. Deze studie omvatte ook de mesh gerelateerde complicaties op korte en lange termijn en werden recent gepubliceerd. ${ }^{17,18}$ In onze studie werd 1 type (niet light) Polypropyleen mesh gebruikt. Onze onderzoekresultaten laten lage implantaat gerelateerde problemen zien. Na gemiddeld 16 maanden werden 166 van de 192 vrouwen die een robotgeassisteerde sacrocolpopexie hadden ondergaan, teruggezien voor inwendig onderzoek met een transparant speculum en vaginaal en rectaal toucher. Twee patiënten werden in deze multicentrische studie geïdentificeerd met een vaginale mesh complicatie, welke poliklinisch behandeld konden worden $(2 / 166 ; 1.2 \%) .{ }^{17}$ Tevens werden 130 vrouwen na sacrocolpo(recto)pexy vier jaar postoperatief opgeroepen voor een controle afspraak. Hiervan werden 96 vrouwen inwendig onderzocht (74\%). Er waren 2 asymptomatische vaginale mesh exposures en 1 symptomatische blaas mesh exposure opgetreden (totaal 3.1\%). ${ }^{18}$ Van de vrouwen die niet werden onderzocht, konden er 22 nog benaderd worden middels een vragenlijst. Geen van deze vrouwen gaf meshgerelateerde klachten aan. Een zeer grote literatuurstudie naar mesh exposure na laparoscopische en robot-geassisteerde sacrocolpopexie, waarbij 65 studies met een minimale follow-up duur van 12 maanden of meer werden geïncludeerd, toonde een mediane mesh exposure van 1.9\% (spreiding 0-13.3\%, spreiding follow-up 12-72 maanden). Hierbij moet worden gemeld dat heterogeniteit tussen de studies hoog was. ${ }^{18}$ 
Complicaties van de ventrale rectopexie zijn laag. In een grote cohortstudie met 919 patiënten die een LVR ondergingen, werd rectale mesh erosie beschreven bij $1.3 \%$ van de patiënten (follow-up duur 10 jaar). ${ }^{10}$

\section{Conclusie}

De FDA/pers en de diverse televisie-uitzendingen waarschuwden destijds terecht voor de mogelijke complicaties van de transvaginale implantatie van matten ter behandeling van vrouwen met prolaps. Helaas is hierdoor de algemene indruk gewekt dat alle soorten niet-lichaamseigen implantaten altijd even slecht zijn. Dit heeft in Nederland ervoor gezorgd dat patiënten met ernstige verzakkingsklachten niet meer naar de dokter durven te gaan. In Engeland is zelfs onder druk van de publieke opinie (tijdelijk) een verbod geplaatst op het gebruik van mesh materiaal. Het Britse NICE (National Institute for Health and Care Excellence) heeft recent o.b.v. wetenschappelijk onderzoek het gebruik onder bepaalde voorwaarden weer toegestaan. ${ }^{19}$

Het is van belang dat bij de introductie van een nieuwe techniek vooraf gedegen onderzoek gedaan wordt naar de mogelijke complicaties. Het is uiteraard noodzakelijk dat in een later stadium, wanneer de operatietechniek klinisch al gebruikt wordt, bij aanwijzingen voor het desondanks ontstaan van mogelijk ernstige complicaties, nader onderzoek gedaan wordt en waarschuwingen afgegeven worden. Echter, de angst voor complicaties is nu gebaseerd op getallen na transvaginale mesh procedures en niet na transabdominale mesh chirurgie. Niet behandelen op basis van complicaties die bij andere typen chirurgie naar voren komen, komt niet ten goede aan de kwaliteit van leven van de patiënt. Ook staat nu het gebruik van mesh materiaal voor andere chirurgische ingrepen, waar vaak jarenlange goede resultaten mee behaald zijn, ter discussie. Goede vervolgafspraken om (mesh-gerelateerde) complicaties in een vroeg stadium te (h)erkennen en behandelen kunnen van toegevoegde waarde zijn.

Bovengenoemde onderzoeken laten zien dat implantaten bij niet-transvaginaal gebruik een heel ander risicoprofiel hebben, en de kwaliteit van leven substantieel kunnen bevorderen. De balans tussen effectiviteit en complicatiekansen moet aantoonbaar ten faveure zijn van de patiënt. Dit moet helder en zonder vooroordeel uitgelegd worden aan de patiënt die voor dit soort ingrepen in aanmerking komt. Patiënten dienen goed geïnformeerd te worden over de effectiviteit en complicatie kansen door het gebruik van niet lichaamseigen materiaal. Op basis daarvan dient vervolgens een zorgvuldige afweging gemaakt te worden door patiënt en behandelaar. De niet complete publieke 
berichtgeving en de negatieve verhalen op internet helpen helaas niet bij dit proces. Het is in het belang van vrouwen met zeer hinderlijke klachten van verzakking om een tegengeluid te laten horen dat gebaseerd is op gedegen vervolgonderzoek bij een grote groep patiënten. Zorgvuldig uitgevoerde transabdominale prolapschirurgie met gebruik van mesh geeft goede lange termijn resultaten, met een laag complicatiepercentage. Bezinning is in dit geval vooral van belang over techniek, niet zozeer over gebruikte materialen.

\section{English summary}

Prolapse of the uterus and vaginal walls is a condition that is commen in women. Surgical repair, because of severe prolapse symptoms is necessary in $11-19 \%$ of women. Unfortunately, recurrence rates are high, estimated around 30\%. To prevent this high recurrence rate, synthetic mesh implants have been introduced to restore the vaginal anatomy through extensive vaginal incisions. This transvaginal surgery was associated with a high number of mesh-related complications. The American Food and Drug Administration published a safety report in 2011 to warn for these mesh-related complications. Reconstructive pelvic floor surgery can also be performed through abdominal surgery, without vaginal incisions. By placing a mesh implant on the prolapsed vaginal vault or rectum, and connect these organs throught the mesh with the sacrum, sufficient results are obtained with a low complication percentage. In the discussion on mesh surgery, often no distinction is made between the surgical techniques (vaginal versus abdominal surgery). As a result, patients with serious pelvic organ prolapse symptoms are not counselled objectively on the different treatment options available. This could leed to undertreatment of severe symptomatic pelvic organ dysfunction. 


\section{Referenties}

1. Maher C, Feiner B, Baessler K, Schmid C. Surgical management of pelvic organ prolapse in women. Cochrane Database of Systematic Reviews. 2013, Issue 4. Art. No.: CD004014.

2. Smith FJ, Holman CDJ, Moorin RE, Tsokos N. Lifetime risk of undergoing surgery for pelvic organ prolapse. Obstet Gynecol. 2010;116:1096-1100.

3. Clark AL, Gregory T, Smith VJ, Edwards R. Epidemiologic evaluation of reoperation for surgically treated pelvic organ prolapse and urinary incontinence. Am J Obstet Gynecol. 2003;189:1261-7.)

4. (2011) FDA. Food and Drug Administration. FDA safety communication: urogynecologic surgical mesh: update on the safety and effectiveness of transvaginal placement for pelvic organ prolapse. Rev Lit Arts Am. Available at: http://www.fda.gov/downloads/medical. July 2011.

5. Haylen BT, Freeman RM, Swift SE, et al. An International Urogynecological Association (IUGA)/International Continence Society (ICS) Joint Terminology and Classification of the complications related directly to the insertion of prostheses (meshes, implants, tapes) and grafts in female pelvic floor surgery. Neurourology and Urodynamics. 2011;30:2-12.

6. https://www.fda.gov/news-events/press-announcements/fda-takes-action-protect-womenshealth-orders-manufacturers-surgical-mesh-intended-transvaginal.

7. Serati M, Bogani G, Sorice P, et al. Robot-assisted sacrocolpopexy for pelvic organ prolapse: A systematic review and meta-analysis of comparative studies. Eur Urol. 2014;66:303-318.

8. Lane FE. Repair of posthysterectomy vaginal-vault prolapse. Obstet Gynecol. 1972;20:72-77.

9. Clifton MM, Pizarro-Berdichevsky J, Goldman HB. Robotic female pelvic floor reconstruction: a review. Urology. 2016;91:33-40.

10. Consten ECJ, van Iersel JJ, Verheijen PM, Broeders IAMJ, Wolthuis AM, D'Hoore A. Long-term outcome after laparoscopic ventral mesh rectopexy. Ann Surg. 2015;262:742-748.

11. Maher CF, Feiner B, DeCuyper EM, Nichlos CJ, Hickey KV, O’Rourke P. Laroscopic sacral colpopexy versus total vaginal mesh for vaginal vault prolapse: a randomized trial. Am J Obstet Gynecol. 2011;204:360.e1-7.

12. Barber MD, Maher C. Apical prolapse. Int Urogynecol J. 2013;24:1815-1833.

13. Pacquée S, Nawapun K, Claerhout F, et al. Long-term assessment of a prospective cohort of patients undergoing laparoscopic sacrocolpopexy. Obstet and Gynecol. 2019;134:323-32.

14. Lee RK, Mottrie A, Payne CK, Waltregny D. A review of the current status of laparoscopic and robot-assisted sacrocolpopexy for pelvic organ prolapse. Eur Urol. 2014;65:1128-1137.

15. Nygaard IE, McCreery, Brubaker L, et al. Abdominal sacrocolpopexy: a comprehensive review. Obstet Gyneacol. 2004;104:805-823.

16. Nygaard I, Brubaker L, Zyczynski H, HM, et al. Long-term outcomes following abdominal sacrocolpopexy for pelvic organ prolapse. JAMA. 2013;309:2016-2024.

17. van Zanten F, van Iersel JJ, Hartog FE, et al. Mesh exposure after robot-assisted laparoscopic pelvic floor surgery: A Prospective Cohort Study. JMIG. 2019;26:636-642.

18. van Zanten F, van Iersel JJ, Paulides TJC, et al. Long-term mesh erosion rate following abdominal robotic reconstructive pelvic floor surgery: a prospective study and overview of the literature. Int Urogyn J. 2019. E-print ahead of journal: doi.org/10.1007/s00192-019-03990-1

19. Urinary incontinence and pelvic organ prolapse in women: management. NICE guideline. Published: 2 April 2019. www.nice.org.uk/guidance/ng123. 




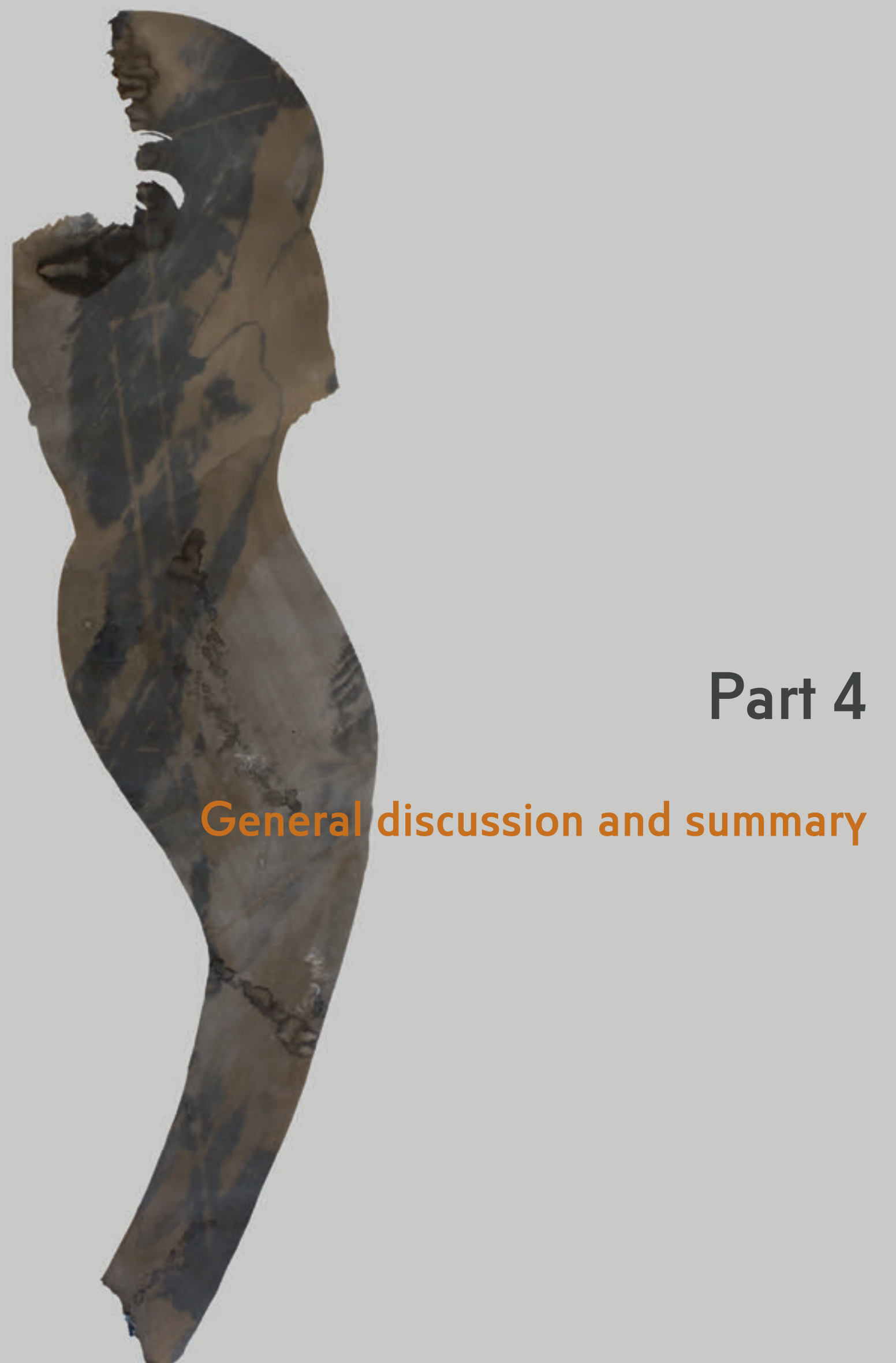





\section{Chapter 10}

\section{General discussion}

and future perspectives 


\section{Main findings}

Synthetic meshes have been used for decades in pelvic reconstructive surgery to reinforce weak or defective supportive tissue. However, the use of synthetic mesh potentially adds to the complication profile and mesh-related morbidity can have a considerable impact on quality of life. Surgical approach has shifted from open surgery to laparoscopic to robotic surgery. This thesis describes results of a large observational cohort study after robot-assisted laparoscopic sacrocolpo(recto)pexy. The findings of the thesis are summarised per section.

\section{Part 1: What are realistic peri- and postoperative outcomes that can be expected when treating patients with robot-assisted laparoscopic sacrocolpopexy, with and without a supracervical hysterectomy?}

\section{Anatomic results}

The results from the bi-centre European cohort performed in this thesis, demonstrated that the robotic approach is an effective and reproducible technique with excellent results associated with the apical compartment (91-99\%). This study included both robot-assisted laparoscopic sacrocolpopexy (RASC) and supracervical hysterectomy with sacrocervicopexy (RSHS). Recurrences, defined as simplified pelvic organ prolapse quantification (sPOPQ) $\geq 2$, were mostly located in the anterior compartment. Theses recurrences occurred both after RASC and RSHS. Less anterior recurrences were symptomatic after RSHS. Regarding all vaginal compartments, the success percentages were $67 \%$ and $65 \%$ for RASC and RSHS respectively. If only recurrences with symptoms of bulge present were considered, anatomic success was 74\% after RASC and $89 \%$ after RSHS. Prolapse-related reinterventions were $23 \%$ after RASC. These interventions mostly consisted of vaginal and/or posterior vaginal wall repairs. After RSHS, the reintervention rate was much lower, $3.7 \%$.

\section{Patient-reported outcomes measurements}

Patient-reported functional outcomes are increasingly becoming the standard of surgical success. The overview of literature in Table 1 in Chapter 1 shows that not all studies describe functional outcomes through validated questionnaires, whereas success of surgery highly depends on these variables. Symptom relief is more important than anatomic restoration. Both quality of life and symptoms of bulge improved significantly 
one year after surgery. Furthermore, sexual function is an important variable, but not yet evaluated extensively. Solving prolapse can improve sexual function, but de novo dysfunction could occur because of shortening the vaginal length or because of mesh complications. A specific validated questionnaire was used to evaluate sexual function. Our cohort showed improvement in sexual function one year after RASC and RSHS evidenced by enhanced physical and emotional scores. The total number of sexually active women and women suffering from dyspareunia before and after surgery did not differ.

\section{Perioperative results}

Intra-operative complications and conversion rates were low (RASC, 5.3 and 4.3\%; RSHS, 0.0 and $0.0 \%$ ). There were significantly more intra-operative complications and conversions observed after RASC than RSHS. The mean duration of surgery was 145 minutes for RASC and 183 minutes for RSHS. Median blood loss per procedure was very low, 25 millimetres after RASC and 50 millimetres after RSHS. Based on 305 procedures, there were five severe (Clavien-Dindo classification $\geq 3$ ) early postoperative complications (1.6\%). Both RASC and RSHS are safe procedures with minimal blood loss.

\section{Learning curve}

Monitoring complications in new techniques is of increasing importance as development in new procedures shifts quickly. Knowing surgical pitfalls may better prepare surgeons. With cumulative sum analysis small shifts in surgical performance can be detected. This can alert surgeons when performance is not optimal. It can also alert a change in patient referral pattern, resulting in more complicated patients with more comorbidity. These analyses can aid in individualising surgical programs. Consensus on surgical training is essential and requirements to begin performing robotic surgery without supervision should be clear. Results from this study indicate that it might be best to perform the first 23-41 cases with an experienced robotic surgeon. Overall, intra-operative complications were infrequent, suggesting implementation of RASC/RSCR to be safe for experienced laparoscopic surgeons. Proficiency regarding intraoperative complications was obtained later, after 78 cases. Surgery time for RASC stabilised much quicker, after 24-29 cases. This suggests that it takes much longer to perform significantly better than the acceptable rate than to lower surgery time. Surgeons should determine if they can perform enough case load to accomplish proficiency. For RSCR, no clear increase or decrease of surgery time was detectable. It implies that surgeon's experience is not the 
sole factor for diminishing surgery time in this procedure. Other factors could contribute, such as team effort. However, this was not investigated in this study.

\section{Robotic technique}

Some question the costs that are related to robotic surgery, since its superiority to laparoscopic surgery is not proven in all fields. The rapid changes make it almost impossible to keep up with well-structured randomized controlled trials to compare all techniques. Objective study results on patient-reported outcome measures, safety of the robotic technique and results on ergonomics are, however, necessary. A different approach in observing learning curves, as described above, could aid in the process of monitoring safety.

It takes time to further develop techniques. In an editorial written by the Editor-in-Chief of 'Obstetrics and Gynaecology' in 1992, he described conventional laparoscopic surgery as a gimmick. Eighteen years later, he withdrew this remark as a result of the benefits of these surgical changes. ${ }^{1,2}$ We now observe developments that result in more compact robotic arms with improved mobility. This enhances faster installing, positively affecting OR time and costs. The console yields improved ergonomics for surgeons. Research on surgeons with musculoskeletal complaints shows that developments in surgical ergonomics are essential and of high priority. ${ }^{3}$ Physical stress can affect surgical performance and result in work leave. This has potential consequences on patient safety and economic costs. With new developments the right surgical instruments for the right surgery can be made, avoiding unnecessary costs. Results of this thesis can aid in answering questions for surgeons that are at the start of introducing a new technique. One of the most important questions surgeons have when starting a new technique is: when am I a competent robotic surgeon? This thesis shows clear intra-operative data on perioperative complications and surgery time, so that surgeons can compare their results per subgroup.

\section{Part 2: What are the long-term results of robot-assisted laparoscopic sacrocolpopexy for vaginal apical prolapse and sacrocolporectopexy for combined pelvic floor disorders?}

\section{Multicompartment prolapse}

Pelvic organ support and its relation to pelvic floor function and dysfunction is complex. Organs in the pelvis are closely connected, and when prolapse in one compartment is 
found, one must be suspicious of prolapse in another compartment. Additional imaging can sometimes be helpful in diagnosing pathology in the pelvis. Acknowledgement of the existence of more than one prolapsed organ is essential and has changed surgical treatment to a multidisciplinary setting over the years. Our results showed at 12 and 48 months after RSCR, recurrence free intervals based on Kaplan Meier estimates of $100 \%$ and $90 \%$ respectively. Patient-reported outcomes, such as symptoms of bulge, fecal incontinence, obstructive defecation, and quality of life improved substantially. However, a part of patients experienced persistent defecation related complaints. This underlies the complexity of treating multicompartment POP.

\section{Long term results for vaginal middle compartment prolapse}

Long-term results after RASC and RSHS shows sustainable results. Ninety-six percent of patients had no apical recurrence. The SPOPQ stages of all four anatomic landmarks improved significantly from pre- to postoperative visit. Similar to our one year results, most recurrences that were found at postoperative visit were (asymptomatic) cystoceles. However, fewer patients received a re-intervention because of prolapse $3 \%$ of patients). For our one year results, we found a $23 \%$ reintervention rate after RASC, mostly consisting of vaginal repairs. This high difference could be explained by the difference of study size, one centre vs bicentre cohort, difference in postoperative indication for repeat surgery among two different European countries and difference in patients' preference regarding retreatment in two different countries (expectative vs surgical management).

Symptoms of bulge, urinary symptoms and quality of life improved substantially. Expectation management plays an important role in the preoperative counselling process. Patients should be counselled about the chance of postoperative (mild) de novo stress urinary incontinence in $20 \%$ of patients, with a $5 \%$ re-intervention rate in the long run.

\section{Part 3: Is the use of mesh implants in sacrocolpopexy safe in the mid- and long-term?}

Both after one year and after four years, a low incidence of mesh exposure was found. The literature overview on minimally invasive sacrocolpopexy (SC) showed also a low median mesh exposure rate $(1.9 \%)$. In this overview solely studies that had a minimal follow-up duration of $\geq 12$ months were included. These results are in line with 
previously published systematic reviews (2-3\%).4,5 More recent publications (followup $\geq 12$ months) showed mesh exposure rates of $1.4-4.5 \%$ for laparoscopic sacrocolpopexy (LSC) and RASC respectively.6-27 The introduction of transvaginal procedures showed a high risk of mesh-related complications (incidence 10.3\%; range, $0-29.7 \%, \mathrm{~N}=11$ 785). ${ }^{28,29}$ This implies that the abdominal use of mesh has a different risk profile than transvaginal mesh surgery. A very recent well designed study, showed that $7 \%$ of women needed a re-intervention due to mesh-related complications after 86 months. ${ }^{30}$ Patients were included starting from 1996, implying that complications could increase with time. Another recent study looked retrospectively at their data as a result of the concerns around mesh usage. They included 660 patients, who underwent LSC between 2005 and 2017 and found five cases (0.7\%) of vaginal mesh exposure and four cases (0.6) of vaginal suture exposure after 4 years and 3 months. The cases were identified from coding data, theatre logs, and the national urogynaecology procedure database. ${ }^{31}$ Difference in mesh exposure after RASC and LSC could exist. As the robotic technique is associated with 3D vision and very low amount of blood loss (median blood loss $25 \mathrm{ml}$, with supracervical hysterectomy $50 \mathrm{ml}$ ), this could prevent hematomas and therefore infection of the mesh implant. Structured follow-up in patients who receive mesh could eventually aid in answering these specific questions.

The debate about the safety of mesh surgery is fierce. Postoperative complications related to mesh-implants are often described without explicitly mentioning subgroups. This cohort study shows that the risk profile of abdominal implants is very different from transvaginal mesh implants. Abdominal use of implants can improve quality of life of women substantially. When you can't use mesh in the treatment of prolapse related complaints, patients either suffer from serious complaints, or could undergo several surgical procedures with their own risks. These considerations should be balanced against each other. Patients should be informed on the efficiency and risk of complications from the use of mesh implants. We aimed to answer the question of the safety of mesh and found a low incidence of complications. This is an encouraging finding for patients with severe POP symptoms and doctors who treat them. However, mesh-related complications can result in severe complaints, implying the need for more research in this field. 


\section{Future research}

\section{Long-term results}

As the incidence of complications is low and after a longer period of time, future research should focus on long-term results with a minimum follow-up of four years, but preferably longer. Women in this thesis had an average age of 60 years. Long-term results are therefore essential to provide these women with a detailed description of the expected outcomes at the long run. A recent study found a higher number of meshrelated reinterventions because of graft-related complications. ${ }^{30}$ Difference in technique (laparoscopic vs robotic), follow-up time and type of mesh used, could explain these differences. Agreements on setting up standard prospective evaluations when introducing new techniques should become standard care. This will take effort and financial investment at the beginning but could avoid unanswered questions and negative results in the longer term. This can not only be the responsibility for clinical working doctors and researchers who often perform this research for free. This should be on the priority list specifically for governmental institutions and professional networks at the national associations for gynaecologists/surgeons and related health professionals.

\section{Robotic surgery}

Technical development and clinical outcomes should become more integrated. In this thesis a narrow collaboration was made between technique and direct clinical outcomes. Not only in surgery, but technical implementations in the whole hospital are evolving (i.e. intensive care unit, emergency care). To integrate these techniques and have efficient clinical outcomes, doctors and technicians should collaborate closely. Although this may sound very logical in the current world surrounded by the newest digital gadgets, in clinical practice this is sometimes far from reality. Improvement in collaboration can improve efficiency, patient-reported outcomes and costs. Special focus can be given to ergonomics for the doctor, aiming to reduce fatigue and improve the health of health care workers. These changes may result in more efficient and better patient care. Medical training can also be improved by ensuring direct feedback of the computer for the trainee. In general, improved technique could enhance better access to medical care by bypassing language barriers or performing surgery from a distance. The opportunities of bringing these two work fields closer together are impossible to overstate. 
Since 2019, the Meander Medical Center, where a big part of this study was conducted, has two robotic consoles: one for the senior surgeon (supervisor) and one for the surgeon in training. The surgeon in training can practice first in a theoretical surgical field. These simulation sessions enhance safety. Direct feedback is given by the computer on which the subject performs her/his training. Examples are more central movements of the surgical instruments, the fluency of movements, time etcetera. During surgery, the supervising surgeon can hand over the controls to the trainee. Specific areas can be pointed out with an arrow to prevent damage of surrounding organs or bleeding of nearby blood vessels.

New developments in robotics are enormous. Feedback from robotic instruments using sensors is now becoming possible. The machine can learn from previous surgeries by using their sensors and aid in informing the surgeon of specific anatomical area's to avoid vessels, nerves etc. Big data will play a major role in this field. The computer can give advice, but the surgeon will still have the final call. In order to prevent damage to healthy tissue, instruments sharing one 'keyhole' incision are being developed and starting to come on the market. Surgical instruments with more freedom of movement at the distal end are also being generated. This can aid gynaecological surgery by performing manoeuvres in the narrow pelvis.

\section{The individual patient}

Preoperative work-up to determine which patient would benefit the most from a certain treatment is essential. One study aimed to determine a preoperative cut-off score to predict improvement after surgery. Postoperative improvement after surgery was nonexistent in women with a preoperative Pelvic Floor Impact Questionnaire (PFIQ-7) score of $<45.25$. Probability of postoperative improvement was $84 \%$ in women with a preoperative PFIQ-7 score of $\geq 45.25$. This supports the use of quality of life questionnaires to select women for LSC, but this method needs further external validation before being used routinely in practice. ${ }^{32}$

Many risk factors have been described for POP and for postoperative recurrence of POP. As there is contradicting evidence, one systematic review included solely risk factors that were significantly associated with POP recurrence in at least two studies. ${ }^{33}$ This study showed preoperative stage as a risk factor for POP recurrence. If more risk factors for recurrent prolapse could be established, a more tailored approach for the patient could be made. This can aid pre-operative decision making. 


\section{Risk factors for mesh exposure}

Preventing mesh-related complications by identifying risk factors could also aid in selecting the right patients for SC. Risk factors for abdominally placed mesh are difficult to identify. The use of polytrafluroethylene (ePTFE) mesh, smoking, total hysterectomy (with opening of the vagina) or stage 3 or 4 prolapse have been reported. ${ }^{34-36}$ Performing a hysterectomy during minimally invasive SC was associated with a 4 times higher risk of mesh exposure compared with no hysterectomy. ${ }^{36}$ Durst et al. aimed to determine if mesh type was an independent predictor for mesh/suture exposure. ${ }^{37}$ Multivariate logistic regression showed prior surgery for incontinence (odds ratio [OR], 2.87; 95\% CI 1.19-6.96), porcine acellular cross-linked collagen matrix with mediumweight polypropylene mesh (OR, 4.95; 95\% CI, 1.70-14.42), heavyweight polypropylene mesh (OR, 6.73; 95\% CI, 1.12-40.63), nonabsorbable braided suture (OR, 4.52; 95\% CI, 1.53-15.37), and immediate perioperative complications (OR, 3.64; 95\% CI, 1.53-13.37) to be independent risk factors. ${ }^{37}$ Few recent studies on light weight mesh reported no mesh exposures. ${ }^{38-40}$ However, the use of light-weight mesh is possibly associated with more recurrences. ${ }^{41}$ In a retrospective study, 461 women underwent RASC with ultralightweight mesh $(\leq 20 \mathrm{~g} / \mathrm{m} 2, \mathrm{n}-248)$ and RASC with heavier weight mesh $(\leq 35 \mathrm{~g} / \mathrm{m} 2,213) .{ }^{41}$ Ultralightweight mesh had twice the risk of failure within 3 years compared to heavier weight mesh (hazard ratio, 2.15; 95\% CI 1.10-4.21; $p=0.03$ ). Among failures, use of ultralightweight mesh was associated with almost 5 times elevated risk of anterior compartment failure. There were less mesh exposures in the ultralightweight mesh group, although this group was followed for less time [ultralightweight: $1.6 \%$, follow-up time 51 days; heavyweight: $6.0 \%$, follow-up 352 days $(p=0.01)]$. Further research should focus on the pathophysiology of heavy and lightweight mesh to improve materials and suggest the perfect weight mesh to prevent both recurrences as mesh-related complications.

\section{Mesh and pain}

Some women describe pelvic pain or lower back pain after surgical repair of their prolapse. As there are many other possible pathophysiologic pathways causing these symptoms, such as back hernia, hypertonic pelvic floor muscles, it would be of interest to further investigate these complaints. Little research has been performed in this area. Pelvic pain, heaviness or discomfort can also be a symptom of mechanical stress of the prolapsing tissues. Underlying conditions that existed already preoperatively, could also cause these symptoms. However, pain complaints can also be related to the way the 
proximal suspension is performed (tackers/stiches placed to the promontory), the mesh itself that has been inserted (shrinkage) into the pelvis or by complications of the surgery. Scoring pain objectively is difficult. Research on chronic pain patients shows that the reliability and validity of pain intensity measurement is best reached by obtaining 3 ratings over 4 days. ${ }^{42}$ An important risk factor for chronic pain is acute or chronic pain at another site. Pain history should therefore be noted preoperatively. Research in this area could play an important role in prevention of pain and should be on the priority list for every researcher working in this field.

\section{Preventive measurements}

The focus of future research should not only be on restoring female POP, but also on preventive measurements. The parauterine ligaments and endopelvic fascia consist of connective tissue. One study investigated the expression of extracellular matrix (ECM) components in fibroblasts when exposed to different sort of strain load. This study concluded that alterations in ECM may be involved in pathogenesis of POP, with decreased synthesis and increased degradation of collagen and elastin. The role of transforming growth factor (TGF- $\beta 1$ ) may play an important part in this process. TGF- $\beta 1$ has a specific value in predicting the severity of POP, but based on these findings, it can possibly also be considered as a therapeutic target. ${ }^{43}$

In recent studies, the alternative of stem cell therapy to classical POP surgery has been investigated. Women with genetic variants/mutations make them more susceptible to the development of POP. Other aetiological factors can influence this process, such as age (transcriptional gene activation and repression), vaginal delivery (accelerating the process) or obesity (mechanical stretching and influencing protein expression). Stem cell therapy could be used in a selected group of women with POP. ${ }^{44}$ These studies show great potential in the treatment of POP symptoms. 


\section{References}

1. Pitkin RM. Operative laparoscopy: surgical advance or technical gimmick. Obstet Gynecol 1992; 79:441-2.

2. Pitkin RM, Parker WH. Operative laparoscopy: a second look after 18 years. Obstet Gynecol 2010; 115:890-1.

3. Alleblas CJC, de Man AM, van den Haak L, Vierhout ME, Jansen FW, Nieboer TE. Prevalence of musculoskeletal disorders among surgeons. Performing minimally invasive surgery. Ann Surg 2017;266:905-920.

4. Lee RK, Mottrie A, Payne CK, Waltregny D. A review of the current status of laparoscopic and robot-assisted sacrocolpopexy for pelvic organ prolapse. Eur Urol. 2014;65:1128-1137.

5. Serati M, Bogani G, Sorice P, Braga A, Torella M, Salvatore S, et al. Robot-assisted sacrocolpopexy for pelvic organ prolapse: a systematic review and meta-analysis of comparative studies. Eur Urol. 2014;66:303-318.

6. Joubert M, Thubert T, Lefranc JP, Vaessen C, Chartier-Kastler É, Deffieux X, et al. Comparison of functional outcomes with purely laparoscopic sacrocolpopexy and robot-assisted sacrocolpopexy in obese women. Progrès en Urologie. 2014;24:1106-1113.

7. Tan-Kim J, Nager CW, Grimes CL, Luber KM, Lukacz ES, Brown HW, et al. A randomized trial of vaginal mesh attachment techniques for minimally invasive sacrocolpopexy. Int Urogynecol J. 2015;26:649-656.

8. Kenton K, Mueller ER, Tarney C, Bresee C, Anger JT. One-year outcomes after minimally invasive sacrocolpopexy. Female Pelvic Med Reconstr Surg. 2016;22:382-384.

9. Linder BJ, Chow GK, Elliott DS. Long-term quality of life outcomes and retreatment rates after robotic sacrocolpopexy. Int J Urol. 2015;22:1155-1158.

10. Myers EM, Siff L, Osmundsen B, Geller E, Matthews CA. Differences in recurrent prolapse at 1 year after total vs supracervical hysterectomy and robotic sacrocolpopexy. Int Urogynecol J. 2015;26:585-589.

11. Jambusaria LH, Murphy M, Lucente VR. One-year functional and anatomic outcomes of robotic sacrocolpopexy versus vaginal extraperitoneal colpopexy with mesh. Female Pelvic Med Reconstr Surg. 2015;21:87-92.

12. Prendergast E, Silver H, Johnson LL, Simon M, Feinglass J, Kielb S, et al. Anatomic outcomes of robotic assisted supracervical hysterectomy and concurrent sacrocolpopexy at a tertiary care institution at initial adaptation of the procedure. Female Pelvic Med Reconstr Surg. 2016;22:2932.

13. Linder BJ, Anand M, Klingele CJ, Trabuco EC, Gebhart JB, Occhino JA. Outcomes of robotic sacrocolpopexy using only absorbable suture for mesh fixation. Female Pelvic Med Reconstr Surg. 2017;23:13-16.

14. Culligan PJ, Salamon C, Priestley JL, Shariati A, Porcine dermis compared with polypropylene mesh for laparoscopic sacrocolpopexy: a randomized controlled trial. Obstet Gynecol. 2013;121:143-151.

15. Rosati M, Bramante S, Bracale U, Pignata G, Azioni G. Efficacy of laparoscopic sacrocervicopexy for apical support of pelvic organ prolapse. JSLS. 2013;17:235-244. 
16. Gracia M, Perelló M, Bataller E, Espuña M, Parellada M, Genís D, et al. Comparison between laparoscopic sacral hysteropexy and subtotal hysterectomy plus cervicopexy in pelvic organ prolapse : a pilot study. Neurourol Urodyn. 2015;34:654-658.

17. Park YH, Yang SC, Park ST, Park SH, Kim HB. Laparoscopic reconstructive surgery is superior to vaginal reconstruction in the pelvic organ prolapse. Int J Med Sci. 2014;11:1082-1088.

18. Sarlos D, Kots L, Ryu G, Schaer G. Long-term follow-up of laparoscopic sacrocolpopexy. Int Urogynecol J. 2014;25:1207-1212.

19. Liu C-K, Tsai CP, Chou MM, Shen PS, Chen GD, Hung YC, et al. A comparative study of laparoscopic sacrocolpopexy and total vaginal mesh procedure using lightweight polypropylene meshes for prolapse repair. Taiwan J Obstet Gynecol. 2014;53:552-558.

20. Li S, Ji M, Zhao Z. The effectiveness of two different laparoscopic surgeries for apical support of pelvic organ prolapse. Eur J Obstet Gynecol Reprod Biol. 2015;188:74-78.

21. El Hamamsy D, Fayyad AM. New onset stress urinary incontinence following laparoscopic sacrocolpopexy and its relation to anatomical outcomes. Int Urogynecol J. 2015;26:1041-1045.

22. Vieillefosse S, Thubert T, Dache A, Hermieu JF, Deffieux X. Satisfaction, quality of life and lumbar pain following laparoscopic sacrocolpopexy: suture vs. tackers. Eur J Obstet Gynecol Reprod Biol. 2015;187:51-56.

23. Costantini E, Mearini L, Lazzeri M, Bini V, Nunzi E, di Biase M, et al. Laparoscopic versus abdominal sacrocolpopexy: a randomized, controlled trial. J Urol. 2016;196:159-165.

24. Dandolu V, Akiyama M, Allenback G, Pathak P. Mesh complications and failure rates after transvaginal mesh repair compared with abdominal or laparoscopic sacrocolpopexy and to native tissue repair in treating apical prolapse. Int Urogynecol J. 2017:28:215-222.

25. Lizee D, Campagna G, Morciano A, Panico G, Ercoli A, Gadonneix P. Laparoscopic sacral colpopexy: how to place the posterior mesh into rectovaginal space? Neurourol Urodyn. 2017;36:1529-1435.

26. Zebede S, Dawood A, Alarab M, Drutz H, Lovatsis D. A streamlined surgical approach to laparoscopic sacrocolpopexy for post-hysterectomy vault prolapse. J Obstet Gynaecol Can. 2016;38:446-452.

27. Chen Y, Hua K. Medium-term outcomes of laparoscopic sacrocolpopexy or sacrohysteropexy versus vaginal sacrospinous ligament fixation for middle compartment prolapse. Int J Gynecol Obstet. 2017;137:164-1.

28. Abed H, Rahn DD, Lowenstein L, Balk EM, Clemons JL, Rogers RG. Incidence and management of graft erosion, wound granulation, and dyspareunia following vaginal prolapse repair with graft materials: A systematic review. Int Urogynecol J. 2011;22:789-798.

29. US Food and Drug Administration. Urogynecologic surgical mesh: update on the safety and effectiveness of transvaginal placement for pelvic organ prolapse. Available at: http://www.fda.gov/downloads/medicaldevices/safety/alertsandnotices/ucm262760.pdf.

30. Pacquée S, Nawapun K, Claerhout F, Werbrouck E, Veldman J, D’hoore A, Wyndaele J, Verguts J, de Ridder D, Deprest J. Long-Term Assessment of a Prospective Cohort of Patients Undergoing Laparoscopic Sacrocolpopexy. Obstet and Gynecol. 2019;134:323-32.

31. Baines G, Price N, Jefferis H, Cartwright R, Jackson SR. Mesh-related complications of laparoscopic sacrocolpopexy. International Urogynecology Journal. 2019;30:1475-1481. 
32. Laas E, Haddad M, Muhlstein J, Bendifallah S, Ballester M, Darai E. Preoperative quality of life questionnaires are an adequate tool to select women with genital prolapse for laparoscopic sacrocolpopexy. Int Urogynecol J. 2017;28:1833-1839.

33. Vergeldt TFM, Weemhoff M, IntHout J, Kluivers KB. Risk factors for pelvic organ prolapse and its recurrence: a systematic review. Int Urogynecol J. 2015;26:1559-1573.

34. Cundiff GW, Varner E, Visco AG, Zyczynski HM, Nager CW, Norton PA, et al. Risk factors for mesh/suture erosion following sacral colpopexy. Am J Obstet Gynecol. 2008;199:688.e1-688.e5.

35. Akyol A, Akca A, Ulker V, Gedikbasi A, Kublay A, Han A, et al. Additional surgical risk factors and patient characteristics for mesh erosion after abdominal sacrocolpopexy. J Obstet Gynaecol Res. 2014;40:1368-1374.

36. Gutman R, Maher C. Uterine-preserving POP surgery. Int Urogynecol J. 2013;24:1803-1813.

37. Durst PJ, Heit MH. Polypropylene mesh predicts mesh/suture exposure after sacrocolpopexy independent of known risk factors: a retrospective case-control study. Female Pelvic Med Reconstr Surg 2017;24: 360-366).

38. Liu C-K, Tsai C-P, Chou M-M, Shen PS, Chen GD, Hung YC, et al. A comparative study of laparoscopic sacrocolpopexy and total vaginal mesh procedure using lightweight polypropylene meshes for prolapse repair. Taiwan J Obstet Gynecol. 2014;53:552-558.

39. Salamon CG, Lewis C, Priestley J, Gurshumov E, Culligan PJ. Prospective study of an ultralightweight polypropylene Y mesh for robotic sacrocolpopexy. Int Urogynecol J. 2013;24:13711375.

40. Culligan PJ, Gurshumov E, Lewis C, Priestley JL, Komar J, Shah N, et al. Subjective and objective results 1 year after robotic sacrocolpopexy using a lightweight Y-mesh. Int Urogynecol J. 2014;25:731-735.

41. Askew AL, Visco AG, Weidner AC, Truong T, Siddiqui NY, Bradley MS. Does mesh weight affect time to failure after robotic-assisted laparoscopic sacrocolpopexy? Female Pelvic Med Reconstr Surg. 2018;00:00-00.

42. Jensen McFarland CA. Increasing the reliability and validity of pain intensity measurement in chronic pain patients. Pain. 1993 Nov;55:195-203.

43. Liu C, Wang Y, Li B-S et al. Role of transforming growth factor $\beta-1$ in the pathogenesis of pelvic organ prolapse: A potential therapeutic target. Int J of molecular med. 2017;40:347-356.

44. Ho MH, Heydarkhan S, Vernet D, Kovanecz I, Ferrini MG, Bhatia NN, Gonzalez-

Cadavid NF: Stimulating vaginal repair in rats through skeletal muscle-derived stem cells seeded on small intestinal submucosal scaffolds. Obstet Gynecol. 2009; 114:300-309. 



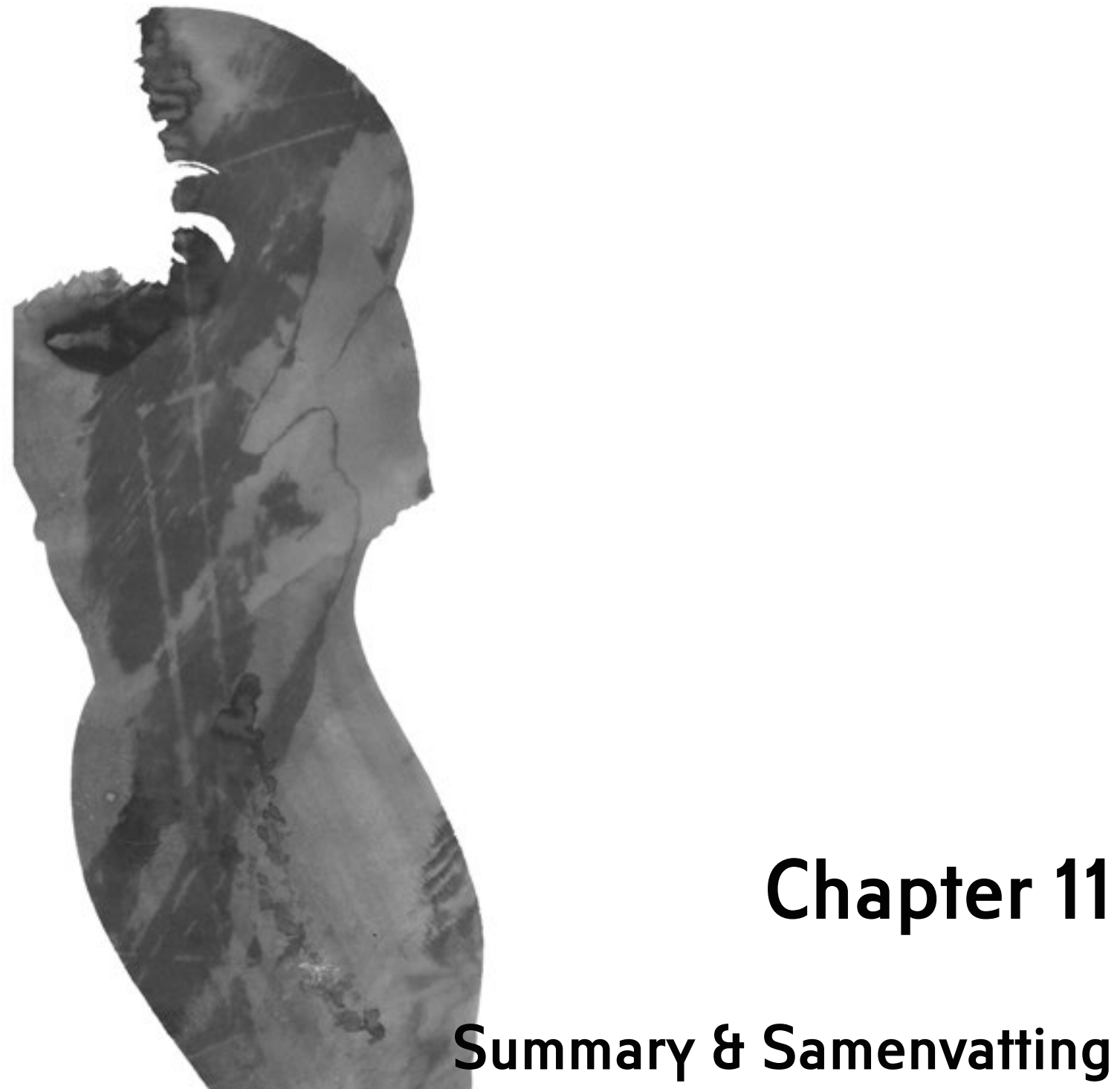




\section{Summary}

In chapter 1, a general introduction on pelvic organ prolapse (POP) and the treatment of POP is provided. Prolapse is a world-wide problem with a high social-economic burden as the number of women with prolapse-related complaints are increasing as a result of aging population. About 1 in 6 women will undergo a surgical treatment because of prolapse or incontinence related symptoms. Sacrocolpopexy (SC) has been used to treat vaginal vault prolapse and is associated with less postoperative recurrences than vaginal sacrospinous colpopexy. In sacrocolpopexy, a mesh implant is used to restore the normal anatomical situation by connecting the prolapsed vaginal apex (and vaginal walls) with the promontory of the sacrum. The procedure can be combined with a supracervical hysterectomy or rectopexy on indication.

In the past decade, robot-assisted surgery has increasingly been used. The optimal ergonomics and improved intracorporeal suturing and 3D vision, aid surgeons in their performance. With robotic assistance, intracorporeal suturing is much easier because the surgeon can make movements with the tip of the instruments that are similar to movements made by his or her own wrists. In contrast to general laparoscopic surgery, or 'straight stick surgery', in which the freedom of movement is much more limited. In general, minimally invasive surgery is characterized by less postoperative pain, shorter hospital stay and quicker return to normal activities with better quality of life. In this thesis, mid- and long-term postoperative patient-reported outcomes, safety of the surgical procedure with robotic assistance and its learning curve were assessed.

Chapter 2 describes perioperative data of a large bicentre European cohort. This cohort included robot-assisted laparoscopic sacrocolpopexy (RASC) and supracervical hysterectomy with sacrocervicopexy (RSHS). The 1-year outcomes on 118 RASCs and 117 RSHSs were reported. Anatomical success of the apical compartment occurred in 91\% (RASC) and in 99\% (RSHS) of patients. If cure of all vaginal compartments was evaluated, the success percentages were lower: $67 \%$ and $65 \%$, respectively. Most recurrences were cystoceles (anterior compartment). Not all recurrences were symptomatic. Twelve percent of women had a symptomatic anterior vaginal wall recurrence after RASC, and 5\% after RSHS. Symptoms of bulge improved from 97.4 to $17.4 \%(p<0.0005)$. Quality of life, measured with the Pelvic Floor Impact Questionnaire (PFIQ-7), improved from $76.7 \pm 62.3$ to $13.5 \pm 31.1(p<0.0005)$. The duration of surgery was significantly longer for RSHS than for RASC (183 minutes versus 145 minutes). 
Intraoperative complications and conversion rates were low (RASC, 5.3 and 4.3\%; RSHS, 0.0 and $0.0 \%$ ). Severe postoperative complications were low after both types of surgery (RASC, 2.1\%; RSHS, 1.6\%). This large prospective cohort study shows that robotassisted apical repair surgery gives durable anatomical results. Both procedures are safe regarding intra- and postoperative complications.

In the hospital where most of this study was conducted, data from two surgeons were monitored from the start of using the robotic approach. These data were used to analyse the learning curve of these two surgeons. This was set out in chapter 3. Determination of the learning curve of new techniques is essential to improve safety and efficiency. Limited information is available regarding learning curves in robot-assisted laparoscopic pelvic floor surgery. Cumulative sum (CUSUM) analysis has been shown to efficiently evaluate surgical performance by detecting small shifts in the parameters of a process. This type of analysis can be used as a self-monitoring tool or to compare results with numbers from the literature. The analysis can mark phases in which complications arise, thereby warning the surgeon to change the training program, or add additional training. We performed a CUSUM analysis for two surgeons during 4 years of surgery. After 78 cases proficiency was obtained. After 24-29 cases, surgery time stabilized for robot-assisted sacrocolpopexy. In this age of rapidly changing surgical techniques, it can be difficult to determine the learning curve of each procedure. Training programs could be individualized with help of CUSUM analysis, improving safety and patient benefits.

Sexual function after prolapse surgery is an underexposed area. In chapter 4 sexual function was examined by a validated questionnaire: the Pelvic Organ Prolapse/Urinary Incontinence Sexual Questionnaire (PISQ-12). Robot-assisted middle compartment surgery improved sexual function 1 year after surgery according to enhanced physical and emotional scores. The total number of sexually active women and complaints of dyspareunia before and after surgery did not differ.

Chapter 5 comprises the long-term results of robotic sacrocolporectopexy (RSCR) for multicompartment prolapse. In RSCR, prolapse of both the vaginal apex and rectum can be restored. Multicompartment prolapses are commonly found, with rates reported of $47 \%$ and higher. Therefore, evaluation of patients with POP by a multidisciplinary team is essential. Ninety percent of patients were recurrence free 48 months after RSCR. Symptoms of vaginal bulge, quality of life, obstructive defecation and faecal incontinence 
improved significantly. However, a subgroup of patients showed persistent bowel complaints, which underlines the complexity of treating multicompartment prolapse.

As female POP is plagued by a high recurrence rate, evaluation of the long-term sustainability of different procedures to treat urogenital prolapse are therefore required. Chapter 6 describes the long-term results after sacrocolpopexy (RASC/RSHS). Seventy-seven patients were included (RASC $n=25$, RSHS $n=52$ ). After 50 months, 96\% of patients showed no apical recurrence. One in nine patients had a symptomatic cystocele. Five percent of patients needed a re-intervention because of recurrent prolapse complaints. Patient-reported outcome measures, such as symptoms of bulge, urinary symptoms and quality of life improved substantially. Preoperatively patients should be counselled on the possibility of 'de novo' stress urinary incontinence. A reintervention because of these complaints can be expected to be around $5 \%$.

The safety of the use of mesh in pelvic floor surgery is matter of debate as result to the occurrence of transvaginal mesh-related complications. The American Food and Drug Administration published a safety report in 2008 and 2011. These warnings were based on the high incidence of mesh-related complications found after surgical use of transvaginal mesh implants (10.3\%). One of the objectives of this thesis was to determine the number of women with mesh exposure (vaginal mesh visualised through separated vaginal epithelium or visualised in other organs) after robotic sacrocolpo(recto)pexy. Mesh exposure can result in severe complaints such as blood loss, pain and infection. Chapter 7 evaluates the mesh exposure rate in a multicentre observational cohort study. After 15.7 months two vaginal mesh exposures were found in 166 patients who underwent RASC or RSHS (1.2\%). Both complications could be treated in the outpatient clinic.

In chapter 8 mesh-related complications after a longer follow-up period were examined. The follow-up was performed in patients in one institution. Both RASC and RSCR were included. A transparent vaginal speculum, proctoscopy, and digital vaginal and rectal examination were used to look for mesh exposure. Seventy-four percent of patients were clinically examined (96/130). Three mesh exposures were diagnosed after a median follow-up time of 48 months (3.1\%; Kaplan-Meier 4.9\%, 95\% confidence interval 0-11.0). Additionally, 22 patients responded solely by questionnaire and/or telephone. They had no mesh-related complaints. An overview of literature, including studies describing mesh exposure after minimal invasive sacrocolpopexy (follow-up $\geq 12$ 
months) was presented. The median mesh exposure rate was $1.9 \%$ (range $0-13.3 \%$, mostly retrospective studies). Based on the current literature and our findings, meshrelated complications seem to be lower in transabdominal mesh surgery than in transvaginal mesh surgery.

To provide Dutch patients and doctors with an overview of these findings, a Dutch editorial was written (chapter 9). The intention of this editorial was to illustrate the current situation on the use of mesh. The use of implants in transabdominal mesh have a different risk profile and can improve quality of life in women with severe prolapse complaints substantially. An overview of literature is also provided in this chapter. Decent follow-up appointments to recognise and treat (mesh-related) complications at an early stage can be of great additional value. Patients should be counselled about the efficiency and risk of surgery with mesh implants after this specific type of surgery. A precise consideration by patient and doctor in deciding their individual treatment plan should then be made. Careful performed transabdominal prolapse surgery with use of mesh implants shows sustainable results regarding patient-reported outcomes and anatomic restoration, with a low complication percentage after four years. 


\section{Samenvatting}

In hoofdstuk 1 wordt een algemene introductie gegeven over het onderwerp verzakking van de bekkenbodem organen (prolaps) en de behandeling hiervan. Prolapsgerelateerde klachten zijn een wereldwijd probleem met een hoge sociaal-economische last, omdat het aantal vrouwen met klachten door een verzakking sterk toeneemt, mede door de vergrijzing. Circa 1 op 6 vrouwen ondergaat een chirurgische behandeling als gevolg van prolapsklachten of urine-incontinentie. Een sacrocolpopexie kan worden verricht bij vrouwen die in het verleden een baarmoederverwijdering hebben ondergaan en zich nu presenteren met een verzakking van de top van de vagina. Deze operatie is geassocieerd met het ontstaan van minder postoperatieve recidieven dan bij vaginale procedures. Via kleine incisies in de buik wordt bij sacrocolpopexie de verzakte vaginatop (en vaginawanden) met behulp van een implantaat weer in de normale anatomische positie gezet en vastgemaakt aan het heiligbeen. De ingreep kan op indicatie gecombineerd worden met een subtotale baarmoederverwijdering of rectopexie (hierbij wordt het laatste deel van de dikke darm ook opgetrokken).

De afgelopen tien jaar wordt robot-geassisteerde chirurgie in toenemende mate gebruikt. De operateur wordt bij robotchirurgie ondersteund door optimale ergonomie en een perfecte visie door 3D en vergroot beeld. Het plaatsen van intracorporele hechtingen (inwendige hechtingen) is veel makkelijker met de robot, doordat bewegingen gemaakt kunnen worden gelijkend op de beweging van de pols. Dit in tegenstelling tot laparoscopische chirurgie of 'kijkoperaties', waarbij de bewegingsvrijheid van het instrumentarium veel minder is. In het algemeen wordt minimaal invasieve chirurgie, zoals robotchirurgie, gekarakteriseerd door minder postoperatieve pijn, kortere opname duur en het sneller kunnen oppakken van de dagelijkse activiteiten. In dit proefschrift worden de mid- en lange-termijn anatomische en functionele resultaten, de intraoperatieve uitkomstmaten en de veiligheid en leercurve van de robot geassisteerde procedure beschreven.

Hoofdstuk 2 beschrijft de perioperatieve data van een groot Europees cohort waarin 2 centra zijn geïncludeerd. Er werden 188 robot-geassisteerde sacrocolpopexiën (RASC) en 117 robot-geassisteerde sacrocolpopexiën met een subtotale baarmoederverwijdering (RSHS) uitgevoerd. Herstel van de anatomie van de top van de vagina was succesvol bij 91\% (RASC) en 99\% (RSHS) van de vrouwen. Als alle vaginale compartimenten beoordeeld werden, dan was herstel lager, namelijk $67 \%$ 
respectievelijk 65\%,. De meeste recidieven betrof een verzakking van de voorwand. Niet alle recidieven gaven klachten. Twaalf procent van de vrouwen had een symptomatische verzakking van de voorwand na RASC en 5\% na RSHS. Klachten van 'balgevoel' verbeterde sterk (van 97\% naar 17\%; $p<0.0005$ ). Kwaliteit van leven, beoordeeld met behulp van de 'Pelvic Floor Impact Questionnaire (PFIQ-7)', verbeterde significant. De duur van de operatie was significant langer voor RSHS dan voor RASC (183 minuten en 145 minuten). Intraoperatieve complicaties en het peroperatief omzetten van de robotoperatie naar open buik chirurgie was laag (RASC, 5.3 en 4.3\%; RSHS, 0.0 en $0.0 \%$ ). Ernstige postoperatieve complicaties waren laag na beide typen chirurgie (RASC, $2.1 \%$; RSHS, 1.6\%). Deze grote, prospectief opgezette studie, laat zien dat robot-geassisteerde reconstructieve bekkenbodemchirurgie om verzakkingen van de top van de vagina te herstellen, goede anatomische resultaten geeft en een forse verbetering van de kwaliteit van leven. Daarnaast zijn beide procedures veilig met betrekking tot intra- en postoperatieve complicaties.

In het ziekenhuis waar het grootste deel van dit onderzoek heeft plaatsgevonden, werd vanaf het begin dat er geopereerd werd met behulp van de robot, data van 2 operateurs bijgehouden. Deze data konden vervolgens gebruikt worden voor het analyseren van een leercurve. De resultaten hiervan zijn te lezen in hoofdstuk 3. Het bepalen van een leercurve voor nieuwe technieken is essentieel en bevordert veiligheid en efficiëntie. Er is echter weinig bekend over het bepalen van leercurven voor robotchirurgie. 'Cumulative sum (CUSUM)' analyse is een techniek waarbij kleine veranderingen in het chirurgisch handelen geobserveerd en ontdekt kunnen worden. De techniek kan gebruikt worden als zelfcontrole, of de eigen resultaten kunnen vergeleken worden met cijfers die bekend zijn in de literatuur. Door middel van de analyse kunnen chirurgische fasen gemarkeerd worden, waarin complicaties optreden. De operateur, en het team, kunnen zo gewaarschuwd worden dat er mogelijk aanvullende of andere training gegeven moet worden. In de analyse uitgevoerd in dit proefschrift werden twee chirurgen over een periode van 4 jaar geëvalueerd. Na 78 ingrepen was 'bekwaamheid' bereikt. Na 24-29 operaties stabiliseerde de operatietijd voor RASC. In deze tijd waarin chirurgische technieken snel veranderen, kan het lastig zijn om een leercurve uit te voeren voor elke procedure. Met behulp van de CUSUM analyse kunnen leerprogramma's gemakkelijker geïndividualiseerd worden, zodat de veiligheid voor patiënten bewaakt wordt. 
Wetenschappelijk onderzoek naar seksuele klachten voor en na prolaps chirurgie is schaars. In hoofdstuk 4 wordt hierop ingegaan. Door middel van een gevalideerde vragenlijst, de 'Pelvic Organ Prolapse/Urinary Incontinence Sexual Questionnaire (PISQ12)', werd deze uitkomstmaat geobjectiveerd. Een jaar na de operatie meldden vrouwen een duidelijke verbetering van de seksualiteit. Dit werd vooral veroorzaakt door verbeterde lichamelijke (opheffen van de verzakking) en emotionele scores. Het totaal aantal seksueel actieve vrouwen en vrouwen met klachten van dyspareunie verschilde niet voor en na de operatie.

In hoofdstuk $\mathbf{5}$ worden de lange-termijn resultaten na robot-geassisteerde sacrocolporectopexie (RSCR) beschreven. Verschillende organen in het bekken kunnen verzakken. Middels een RSCR kan een verzakking van zowel de vaginatop als de endeldarm opgeheven worden. Een verzakking op meer dan één plek in de bekkenbodem, komt regelmatig voor. In de literatuur worden percentages van $47 \%$ of hoger beschreven. Zorgvuldige evaluatie van patiënten met verzakkingsklachten door een multidisciplinair team van chirurgen, gynaecologen en urologen is daarom noodzakelijk. Na 48 maanden, was $90 \%$ van de patiënten recidief vrij, gebaseerd op Kaplan Meier bepalingen. Symptomen van balgevoel door de verzakking, kwaliteit van leven, obstructieve defecatie en fecale incontinentie verbeterden significant. Een deel van de patiënten toonde persisterende darmklachten. Dit onderstreept de complexiteit van het behandelen van verzakkingen van meerdere bekkenbodemorganen.

Het aantal recidieven na operaties voor vaginale prolaps ligt hoog. Om deze reden is evaluatie van de lange termijn resultaten noodzakelijk. Hoofdstuk 6 beschrijft de lange termijn resultaten na behandeling met sacrocolpopexie (RASC/RSHS). Om dit te onderzoeken werden 77 patiënten geïncludeerd (RASC n=25, RSHS n=52). Na 50 maanden had 96\% van de vrouwen een goed herstelde anatomie van het middelste deel van de vagina. Circa 1 op 9 vrouwen had een symptomatische cystocele (verzakking van de vaginavoorwand met de blaas). Drie procent van de vrouwen had een heroperatie nodig vanwege hernieuwde verzakkingsklachten. Klachten die specifiek gerapporteerd werden door patiënten, zoals balgevoel, mictieklachten en kwaliteit van leven, verbeterden substantieel. Preoperatief bespreekt de arts met de patiënt welke klachten mogelijk na een operatie kunnen ontstaan. Door herstel van de anatomie van de blaas kan na RASC/RSHS klachten van stress urine-incontinentie (verlies van urine bij druk verhogende momenten) ontstaan. Een heroperatie in verband met klachten van stress urine-incontinentie lag rond de $5 \%$. 
De veiligheid van implantaten in bekkenbodemchirurgie staat ter discussie door het optreden van complicaties na transvaginale mesh chirurgie. De Amerikaanse Food and Drug Administration publiceerde een veiligheidsrapport hierover in 2008 en 2011. Hierin waarschuwden ze voor de hoge incidentie van complicaties na het chirurgisch plaatsen van transvaginale mesh implantaten (10.3\%). Eén van de doelen van dit proefschrift was om het aantal vrouwen met 'mesh expositie' (blootliggen of doorschemeren van het implantaat door de vaginawand of door andere organen) na robot-geassisteerde sacrocolpo(recto)pexie vast te stellen. Mesh expositie kan resulteren in (ernstige) klachten, zoals bijvoorbeeld bloedverlies, pijn of infectie. Hoofdstuk 7 beschrijft het aantal vrouwen met mesh exposities in een multicentrisch observationeel cohort. Na een follow-up duur van bijna 16 maanden werden twee vaginale mesh exposities gevonden in de 166 onderzochte vrouwen die RASC of RSHS hadden ondergaan (1.2\%). Beide exposities konden poliklinisch verholpen worden.

In hoofdstuk 8 worden de gevonden mesh gerelateerde complicaties na een langer tijdsbestek beschreven. Deze studie was verricht in één centrum. Zowel RASC en RSCR procedures werden geïncludeerd. Een transparant speculum, proctoscoop en een vaginaal en rectaal toucher werd toegepast om mesh expositie te kunnen vinden. Zevenenzeventig procent van de patiënten werd klinisch onderzocht (96/130). Drie mesh exposities werden gevonden in een periode van 48 maanden (Kaplan-Meier 4.9\%, 95\% betrouwbaarheids interval 0-11.0). Aanvullend werden 22 patiënten die niet waren gekomen voor klinisch onderzoek beoordeeld middels papieren vragenlijsten en/of telefonisch. Geen van deze patiënten rapporteerden mesh-gerelateerde klachten. Een overzicht van studies die mesh exposities beschrijven bij patiënten die gedurende minimaal een jaar na de operatie gevolgd werden, werd gepresenteerd in ditzelfde hoofdstuk. De meeste studies die geïncludeerd werden, waren retrospectieve studies. Het mediane percentage mesh expositie lag op 1.9\% (range 0-13.3\%). Gebaseerd op deze studies en onze bevindingen, lijkt het aantal mesh-gerelateerde complicaties lager na transabdominale chirurgie ten opzichte van transvaginale chirurgie.

Om Nederlandse patiënten en dokters een overzicht te presenteren van deze bevindingen, werd een Nederlands artikel geschreven. Dit artikel is te lezen in hoofdstuk 9 en had als doel om de huidige situatie rondom het gebruik van mesh toe te lichten. Hierin werd het verschil in risicoprofiel tussen transabdominale mesh en transvaginale mesh benadrukt. Implantaten bij niet-transvaginaal gebruik hebben een heel ander risicoprofiel, en kunnen de kwaliteit van leven bij vrouwen met ernstige 
prolapsklachten substantieel bevorderen. Een overzicht van de beschreven literatuur staat in dit hoofdstuk vermeld. Goede vervolgafspraken om (mesh-gerelateerde) complicaties in een vroeg maar zeker ook in een later stadium te (h)erkennen en behandelen kunnen van toegevoegde waarde zijn. Patiënten dienen goed geïnformeerd te worden over de effectiviteit en complicatie kansen door het gebruik van niet lichaamseigen materiaal, per specifieke chirurgische procedure. Op basis daarvan dient vervolgens een zorgvuldige afweging gemaakt te worden door patiënt en behandelaar. Zorgvuldig uitgevoerde transabdominale prolapschirurgie met gebruik van mesh geeft goede lange termijn resultaten met betrekking tot patiënt gerapporteerde tevredenheid en anatomisch herstel, met een laag complicatiepercentage na vier jaar. 




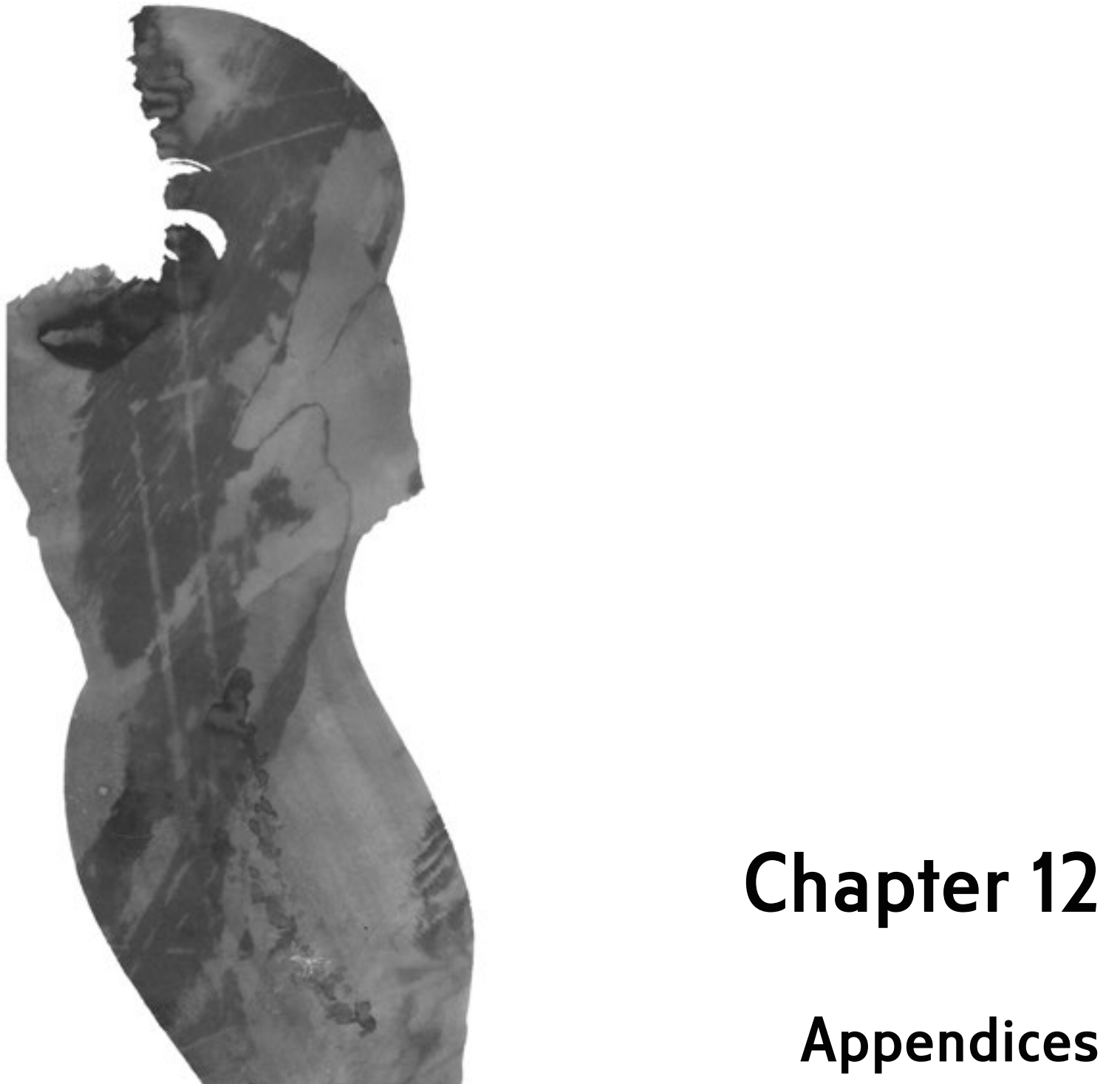

Questionnaires A. Abbreviations B. Acknowledgements - Dankwoord C.

Curriculum Vitae D. List of Publications E. PhD Doctoral Education Program F. 


\section{A. Questionnaires}

UDI-6. The Urogenital Distress Inventory Short Form

Do you experience, and if so, how much are you bothered by:

1. Frequent urination

2. Leakage related to feeling of urgency

3. Leakage realted to activity, coughing, or sneezing

4. Small amounts of leakage (drops)

5. Difficulty emptying bladder

6. Pain or discomfort in lower abdominal or genital area

Scoring 0 for not at all, 1 for slightly, 2 moderately, 3 for greatly.

Domains of the UDI-6: question $1+2=$ irritative symptoms, question $3+4=$ stress symptoms, question $5+6=$ obstructive/discomfort symptoms. The validated Dutch version of this questionnaire was used in this thesis. 
PFIQ-7. Pelvic Floor Impact Questionnaire

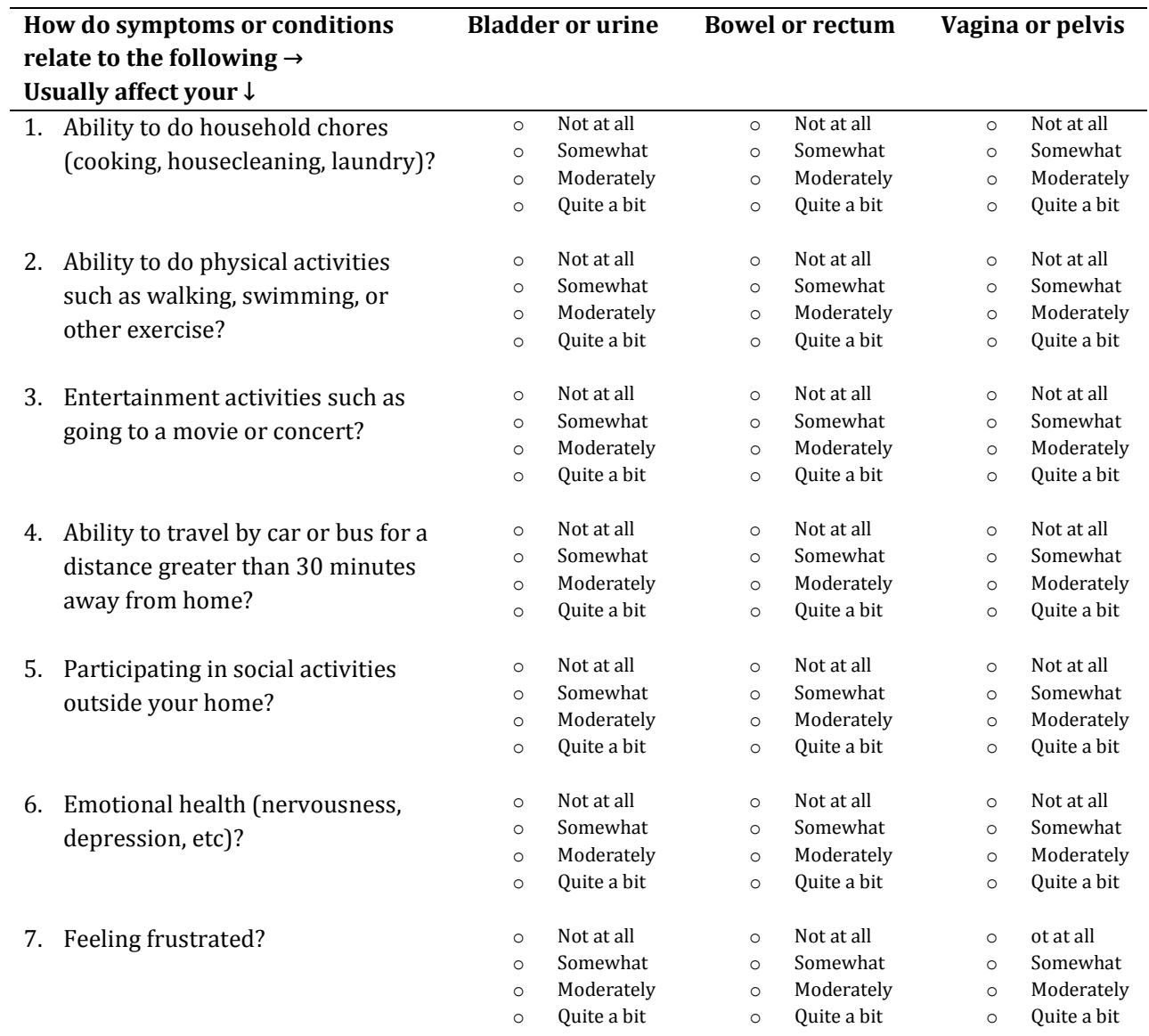

Instructions some women find that bladder, bowel, or vaginal symptoms affect their activities, relationships, and feelings. For each question place an $\mathrm{X}$ in the response that best describes how much your activities, relationships, or feelings have been affected by your bladder, bowel, or vaginal symptoms or conditions over the last 3 months.

Scoring 0 , Not at all; 1 , somewhat; 2 , moderately; 3 , quite a bit. Obtain the mean value for all of the answered items within the corresponding scale (possible value $0-3$ ) and then multiply by $(100 / 3)$ to obtain the scale score (Range 0-100). Missing items are dealt with by using the mean from answered items only. Summary score: add the scores from the 3 scales together to obtain the summary score (range 0-300). The validated Dutch version of this questionnaire was used in this thesis. 
PISQ-12 The pelvic organ prolapse/urinary incontinence sexual questionnaire

1. How frequently do you feel sexual desire? This feeling may include wanting to have sex, planning to have sex, feeling frustrated due to lack of sex, etc.

2. Do you have an orgasm when having sexual intercourse with your partner?

3. Do you feel sexually excited (turned on) when having sexual activity with your partner?

4. How satisfied are you with the variety of sexual activities in your current sex life?

5. Do you feel pain during sexual intercourse?

6. Are you incontinent of urine (leak urine) with sexual activity?

7. Does fear of incontinence (either stool or urine) restrict your sexual activity?

8. Do you avoid sexual intercourse because of bulging in the vagina (either the bladder, rectum, or vagina falling out)?

9. When you have sexual intercourse with your partner, do you have negative emotional reactions such as fear, disgust, shame or guilt?

10. Does your partner have a problem with erections that affects your sexual activity?

11. Does your partner have a problem with premature ejaculation that affects your sexual activity?

12. Compared to orgasms you have had in the past, how intense are the orgasms you have had in the past six months?

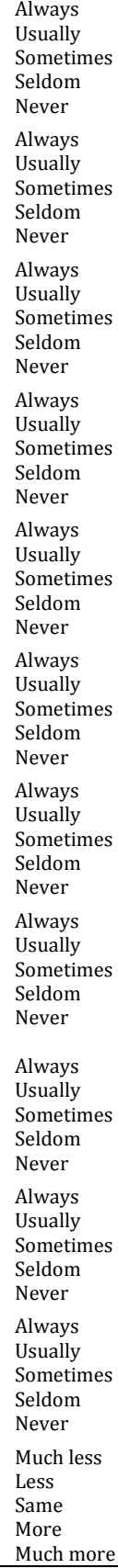

Scoring scores are calculated by totalling the scores for each question with $0=$ never, $4=$ always. Reverse scoring is used for items 1,2,3, and 4 . The validated Dutch version was used for this thesis. 


\section{B. Abbreviations}

$\begin{array}{ll}\text { BMI } & \text { Body Mass Index } \\ \text { CI } & \text { Confidence Interval } \\ \text { PARSEC } & \text { Prospective Assessment of Robotic Sacrocolpopexy: a } \\ & \text { European Bi-centre Cohort } \\ \text { PISQ-12 } & \text { Pelvic Organ Prolapse/Urinary Incontinence Sexual } \\ & \text { Questionnaire } \\ \text { PFIQ-7 } & \text { Pelvic Floor Impact Questionnaire } \\ \text { POP } & \text { Pelvic organ prolaps } \\ \text { POPQ } & \text { Pelvic organ prolapse quantification system } \\ \text { QoL } & \text { Quality of Life } \\ \text { RASC } & \text { Robot-asssisted laparoscopic sacrocolpopexy } \\ \text { RCT } & \text { Randomised controlled trial } \\ \text { RSCR } & \text { Robot-asssisted laparoscopic sacrocolporectopexy } \\ \text { RSHS } & \text { Robot-assisted supracervical hysterectomy with } \\ \text { SD } & \text { sacrocervicopexy } \\ \text { SPOPQ } & \text { Standard deviation } \\ \text { SUI } & \text { Simplified pelvic organ prolapse quantification system (short } \\ \text { TVT } & \text { form) } \\ \text { UDI-6 } & \text { Stress urinary incontinence } \\ \text { UUI } & \text { Tension-free Vaginal Tape } \\ \text { U.S. FDA } & \text { Urogenital Distress Inventory short form } \\ & \text { Urge urinary incontinence } \\ & \text { American Food and Drug Administration } \\ & \end{array}$




\section{Acknowledgements - Dankwoord}

De studies in dit proefschrift zijn tot stand gekomen door de medewerking van een groot aantal mensen. Graag wil ik hen via deze weg bedanken voor al hun inzet, tijd en aanmoedigingen. Allereerst, ben ik alle patiënten erg dankbaar omdat zij tijd hebben besteed aan het invullen van de vragenlijsten. Zonder hen had ik deze studies niet kunnen afronden.

Prof. dr. I.A.M.J. Broeders, beste Ivo, ik vond het fantastisch om mijn promotie bij jou te mogen uitvoeren. Jouw kracht om in een paar woorden structuur te brengen aan de opzet van een artikel hebben mij erg geholpen. Met humoristische opmerkingen op mijn waterval aan vragen, kon ik vervolgens snel beslissingen nemen. Het enthousiasme waarmee jij de technische en de medische wereld dicht bij elkaar weet te brengen, is erg inspirerend. Als AIOS gynaecologie kwam ik je ineens vaker tegen op de operatiekamers, wat het overlegmoment een stuk efficiënter maakte en waardoor ik je beter heb leren kennen. Bedankt voor de fijne samenwerking van de afgelopen jaren. Je hebt mijn horizon met betrekking tot innovatie sterk verbreed!

Dr. S.E. Schraffordt Koops, beste Steven, jouw voorstel om een promotietraject aan te gaan bij de technische Universiteit Twente, zag ik als een grote kans. Jij wist toen nog niet dat ik op 18-jarige leeftijd erg had getwijfeld tussen de studie geneeskunde en een technische universitaire opleiding. De combinatie van technisch en medisch onderzoek was me dan ook op het lijf geschreven. Bedankt voor alle tijd en energie die je in dit project hebt gestopt. Ik ken niemand die zo bevlogen en betrokken is als jou! Ik kon je altijd bellen met wetenschappelijke vragen, ook al zat je op een zeilboot in Friesland! Jouw enthousiasme om aan elk congres deel te nemen, zorgde voor een paar leuke gezamenlijke trips, waarvan Barcelona het hoogtepunt was. Scheurend met de taxi door het centrum van Barca, om op tijd aan te schuiven voor de cerveza. Daarnaast zal ik onze (ethische) discussies niet snel vergeten! Naast al het werk in de kliniek, heb je me (met veel, lees heel veel, grapjes) gesteund om dit onderzoek tot een goed einde te brengen, waarvoor heel veel dank!

I would like to thank the following gynaecologists and surgeons for all their effort and dedication. Without your help, I could not have fulfilled this project. Thank you prof. dr. E.C.J. Consten, dr. P.M. Verheijen, prof. dr. B.A. O’Reilly, dr. H.W.R. Schreuder, dr. O.E. O’Sullivan, dr. S. O’Sullivan, dr. F.E. Hartog and dr. C.I.D. Aalders. 
Prof. dr. M.Y. Bongers, prof. dr. E.C.J. Consten, prof. dr. B.A. O’Reilly, dr. H.W.R. Schreuder, prof. dr. ir. Stramigioli, prof. dr. C.H. van der Vaart, prof. dr. ir. R.M. Verdaasdonk, thank you all very much for participating as a member in the graduation committee. I would like to thank prof. dr. J.N. Kok as chair of the committee.

Drs. Lenters, beste Egbert, bedankt voor al je inzet om aan voldoende studiemateriaal te komen. Door de jaren heen heb ik al veel met je kunnen opereren en dat is altijd een feestje! Dr. Jan van Iersel, drs. Cherèl Brem en drs. Emma van der Schans, Bij jullie had ik echt een teamgevoel: samen schouders eronder en een mooie publicatie maken. Bedankt daarvoor! Beste Corinne Verduijn-Staal, Mariëlle Postma en Marjolein Langemeijer, jullie mogen je echt het 'dedicated robot team' noemen! Corinne op de poli, Mariëlle en Marjolein op de OK. Bedankt voor alle organisatie en gezelligheid.

Dr. Pieternel Pasker-de Jong wil ik graag bedanken voor alle adviezen rondom de statistiek van dit project.

Lieve Eveline en Sophie, lieve paranimfen, wat hebben wij al veel samen meegemaakt. Twee filosofen, 1 arts, 1 promovenda, maar vooral twee hele goede vriendinnen. Wat fijn dat ik jullie in mijn team heb als paranimfen. Jullie zijn toppers!

Beste Elisabeth, Egbert Brinkhuis, Arien, Jitze, Inge, Kees, Egbert Lenters, Anna, Paulien, Steven, Willem, Jolande, Wim en Marjolein, gynaecologen van het Meander Medisch Centrum, jullie zijn een geweldig team! Bedankt voor al jullie positieve steun en geloof in mij. Ik heb door dit proefschrift de kans gekregen om langer met jullie samen te mogen werken en ik mocht zelfs als AIOS bij jullie verder leren en groeien! Jullie dragen stuk voor stuk bij aan mijn opleiding en zijn altijd betrokken. Bedankt voor de mooie reis die ik heb mogen maken naar Vancouver om daar te spreken op het IUGA congres.

Lieve (oud) collega arts-assistenten van het Meander Medisch Centrum, wat was ik altijd blij als ik even met jullie kon kletsen op de dagen dat ik in ons 'hok' zat te werken. Een koffietje, 15 minuten in de zon zitten, een grappig appje, het hielp me enorm. Ik ben zo blij met jullie als mijn collega's en we vormen samen een hechte groep! 
Beste verloskundigen en verpleegkundigen obstetrie van het Meander Medisch Centrum, bedankt voor alle (nacht)diensten waarin ik aan het proefschrift zat te werken en mijn hart bij jullie mocht luchten over alle regeltjes en referentiechecks (met name het controleren van referenties was een goede nachttaak aangezien daar een geringe continue dosis koffie voor nodig is).

Dr. A.J. Goverde, opleider in het UMC Utrecht, beste Angelique, bedankt voor het vertrouwen en de kans om als AIOS in Amersfoort te beginnen. Hierdoor heb ik de opleiding tot gynaecoloog en promotie beter kunnen combineren en afmaken.

Lieve Eveline, Eleá, Leonard en Merel, met jullie heb ik lange dagen in de bibliotheek van Amsterdam gesleten. Wat een motivatie gaf het mij om samen met jullie te kunnen werken. Hierdoor was het werken in de kliniek met het promotieonderzoek veel beter te combineren en mede door jullie heb ik de eindstreep kunnen halen! Lieve Nienke en Charlotte, bedankt voor alle mooie gesprekken over het leven!

Lieve Rie, wat fijn dat ik één van jouw prachtige schilderijen heb mogen gebruiken voor de omslag van dit proefschrift. Voor mij staat deze omslag voor vrouwelijkheid en indirect voor het verbeteren van de gezondheid van vrouwen.

Dear Marlise, we met in Nepal, where I learned to speak English very fast, as to keep up with your enthusiastic and speedy sentences. The trip to Everest Basecamp was a trip of a life time and the base for our friendship. Thank you very much for all the help with language editing and with providing me insight in scientific English writing.

Lieve basketbalstrijders! Drie keer per week samen trainen en wedstrijden spelen. Vijf minuten op het veld en ik was mijn hele werkdag weer vergeten. Zoveel gelachen met jullie en alles gegeven. Dat teamgevoel is het beste gevoel ter wereld. Zonder die energie had ik dit traject niet kunnen afmaken. Lieve Siep in het speciaal. De dag dat we het nieuws kregen dat je niet meer met ons zou spelen, zal ik nooit vergeten. De gesprekken op de fiets naar trainen over werk en privé en wat dat voor ons betekende, zullen me altijd bijblijven. Als de balans hierin dreigde door te schieten, heb ik vaak met een grote glimlach aan deze warme momenten teruggedacht. 
Lieve Marja, Sietse, Johan en Nina, bedankt voor al jullie steun en aanmoediging. Marja, jou wil ik in het bijzonder bedanken. Op de eerste plaats met het helpen ontwerpen van het proefschrift. Zonder jouw Photoshop kwaliteiten was het nooit gelukt. Een proefschrift maken in Ballorig, dat is toch wel het ultieme toppunt van multitasken! Maar ik wil je vooral bedanken voor het luisterend oor dat je me altijd biedt.

Lieve Ina en Wim, zonder jullie onvoorwaardelijke steun had ik nooit deze promotie kunnen doen. Hard werken, willen doorleren, maar vooral ook praktisch en concreet blijven, heb ik niet van vreemden. Door jullie heb ik altijd willen en kunnen doorleren. Ina, fijn dat je altijd de tijd nam om te luisteren naar mijn ideeën. Jouw eigen weg in jouw studiejaren hebben mij heel erg geïnspireerd om door te leren. Wim, het was heel leuk om samen figuren te maken. Ze zijn voor mij de pronkstukjes van mijn proefschrift. Vroeger tekenden we samen gewoon huizen. Nooit gedacht dat we ooit samen een bekkenbodem zouden tekenen, in autoCAD nog wel. Gelukkig is dat programma wel in lijn met de technische universiteit.

Lieve Reginald, het zit erop! Dat is iets dat we nu samen kunnen zeggen. Het was een hele reis, voor ons allebei. Zonder jou 'ik haal je wel even op', waar dan ook, was dit proefschrift er nooit geweest. Bedankt dat je altijd achter me staat en voor alle liefde die je mij geeft. We hebben door dit traject mooie gesprekken gevoerd en ervaringen gedeeld. Lijken we toch veel meer op elkaar dan we dachten! Ik zie het leven als een avontuurlijke reis en met jou en Roselynn hoop ik nog veel mooie reizen te mogen maken. 


\section{Curriculum Vitae}

Femke van Zanten was born on August 22th 1988 in Zaanstad, the Netherlands, as the youngest of three children. In 2006 she graduated at the Zaanlands Lyceum in Zaandam and started with her medical degree at the University of Amsterdam. Before starting her internships, she travelled for one year from Nepal to Japan, working at different hospitals to gain medical experience. Due to an interest in both the surgical and gynaecological field, she ended her medical study with internships in these two areas. Global health was one of her interests as well. Therefore she decided to spend another half a year in Argentina to work at the surgical ward and to study Spanish. In 2014, she started as a surgical resident at the St. Antonius Hospital in Nieuwegein. Because of her rising interest for gynaecology and obstetrics, she continued to work as a resident at the department of gynaecology and obstetrics at the Meander Medical Centre in Amersfoort. In 2016 she combined her residency with academic research. The majority of her studies were presented at both national and international conferences. In 2018 she started her gynaecological training program at the University Medical Centre Utrecht (supervisor: Dr. A.J. Goverde). Besides her work in the field of medicine, sports are a big priority for her. In her free time she playes basketball every week. She lives together with Reginald George and Roselynn in Amsterdam. 


\section{E. List of Publications}

van Zanten F, Schraffordt Koops SE, O'Sullivan OE, Lenters E, Broeders IAMJ, O'Reilly BA. Robot-assisted surgery for the management of apical prolapse: a bi-centre prospective cohort study. BJOG 2019;126:1065-1073.

van Zanten F, Schraffordt Koops SE, Pasker-de Jong PCM, Lenters E, Schreuder HWR. Learning curve of robot-assisted laparoscopic sacrocolpo(recto)pexy: a cumulative sum analysis. Am J Obstet Gynecol. 2019;221:483.e1-11.

van Zanten F, Brem C, Lenters E, Broeders IAMJ, Schraffordt Koops SE. Sexual function after robot-assisted prolapse surgery: a prospective study. Int Urogynecol J 2018;29:905-912.

van Zanten F, van Iersel JJ, Hartog FE, Aalders KIM, Lenters E, Broeders IAMJ, Schraffordt Koops SE. Mesh Exposure After Robot-Assisted Laparoscopic Pelvic Floor Surgery: A Prospective Cohort Study. Journal of Minimally Invasive Gynecology. 2019;26:636-642.

van Zanten F, van Iersel JJ, Paulides TJC, Consten ECJ, Lenters E, Verheijen P, Broeders IAMJ, Schraffordt Koops SE. Long-term mesh erosion rate following abdominal robotic reconstructive pelvic floor surgery: a prospective study and overview of the literature. Int Urogyn J. 2019;E-print ahead of journal. Doi: 10.1007/s00192-019-03990-1.

van Zanten F, van der Schans EM, Consten ECJ, Verheijen PM, Lenters E, Broeders IAMJ, Schraffordt Koops SE. Long-term anatomical and functional results of robot-assisted pelvic floor surgery for the management of multicompartment prolapse: a prospective study. Diseases of the Colon \& Rectum, in print. 


\section{F. PhD Doctoral Education Program}

Twente Graduate School 'PhD Education Certificate'

\begin{tabular}{llr}
\hline Deepening, Broadening \& Academic Skills & Organizer & EC \\
\hline Courses & & 0,50 \\
Team training in a simulation setting for acute & Meander Medical Centre \\
obstetric emergencies and Basic Life Support & & 1,50 \\
(2018) & University of Twente & 1,00 \\
Academic publishing bootcamp (2018) & University of Twente & 0,50 \\
Academic presentations bootcamp (2018) & University of Twente & 0,50 \\
Data management bootcamp (2018) & University of Twente & 1,00 \\
Scientific information bootcamp (2018) & University of Twente & 1,50 \\
TGS Introduction Workshop. (2018) & University Medical Centre Utrecht &
\end{tabular}

\section{Trainings}

Obstetrics cardiotocography course (2018)

Maastricht University Medical Centre $\quad 0,50$

E-learning surgical robot (2018)

Intuitive surgical $\quad 0,50$

Hands on work-shop on diagnosis and repair of 3rd/4th degree obstetric tears (2018)

COBRA: Gynaecological surgical training course (2018)

Newborn life support (2017)

International urogynecological association; $\quad 0,50$ IUGA

Chirurgische Opleiding en Bijscholing $\quad 0,50$

Randstad Academie

Meander Medical Centre $\quad 0.50$

\section{Seminars}

Presentation on pelvic floor surgery with surgical NVOG workgroup pelvic floor recording (2016)

Seminar/oral podium presentation: 'One-year results and safety of robot-assisted

Society of European Robotic Gynaecology

sacrocolpo(recto)pexy' (2016)

Seminar/oral podium presentation: 'Robotassisted sacrocolpopexy for the treatment of Surgery; SERGS

European society for Gynaecological pelvic organ prolapse, a multicentre prospective analysis of pelvic floor function' (2016) VAGO Conference for physicians in training (2017)

Seminar/oral podium presentation: Endoscopy; ESGE.

Vereniging Assistenten Gynaecologie en 0,50 Obstetrie.

Meander Medical Centre 1,50

'Mictieklachten voor en na robot geassisteerde prolaps chirurgie; een prospectieve analyse' (2017) 
Twente Graduate School 'PhD Education Certificate' Continued

\begin{tabular}{|c|c|c|}
\hline Seminar/oral podium presentation: 'Mesh erosie & Nederlandse Vereniging voor & 1,50 \\
\hline na robot geassisteerde sacrocolpopexie' (2017) & Endoscopische Chirurgie; NVEC & \\
\hline Seminar and oral podium presentation & International urogynecological association; & 3,00 \\
\hline 'Prospective assessment of robotic assisted & IUGA & \\
\hline sacrocolpopexy for the treatment of pelvic organ & & \\
\hline prolapse; a European bi-centre cohort' (2017) & & \\
\hline Poster presentation (2017) & International Continence Society; & 0,50 \\
\hline & ICS & \\
\hline Teaching/Student supervision & & \\
\hline Internship supervisor (2018) & Meander Medical Centre/ University & 4,00 \\
\hline & Medical Centre Utrecht & \\
\hline Robotic endoscopic surgery (2018) & Meander Medical Centre/ & 0,50 \\
\hline & University of Twente & \\
\hline Exemptions & & \\
\hline Registration system for surgical complications & Meander Medical Centre & 5,00 \\
\hline$(2016-2019)$ & & \\
\hline
\end{tabular}



